Workshop

Report

Dynamic Processes in Biology,

Chemistry, and Materials Science:

Opportunities for UltraFast

Transmission Electron Microscopy

A report from the EMSL Ultrafast Transmission Electron Microscopy workshop, June 14-15, 2011

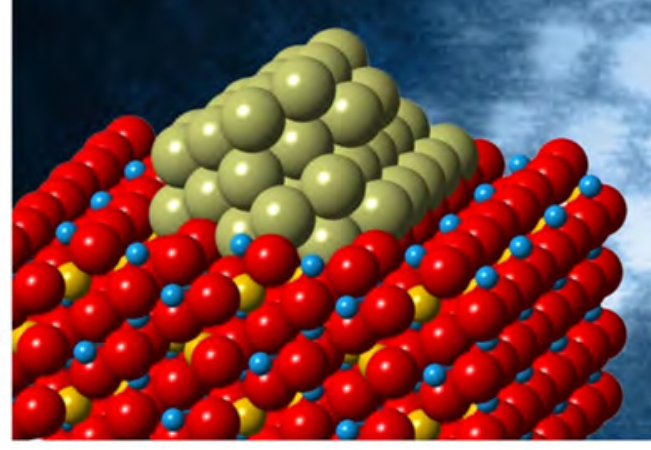

$2.7 \mathrm{fs}$

$6.7 \mathrm{fs}$

s
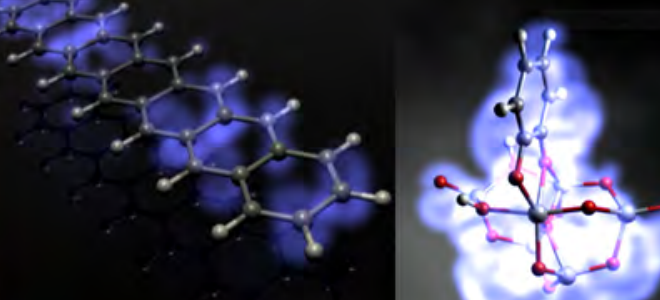

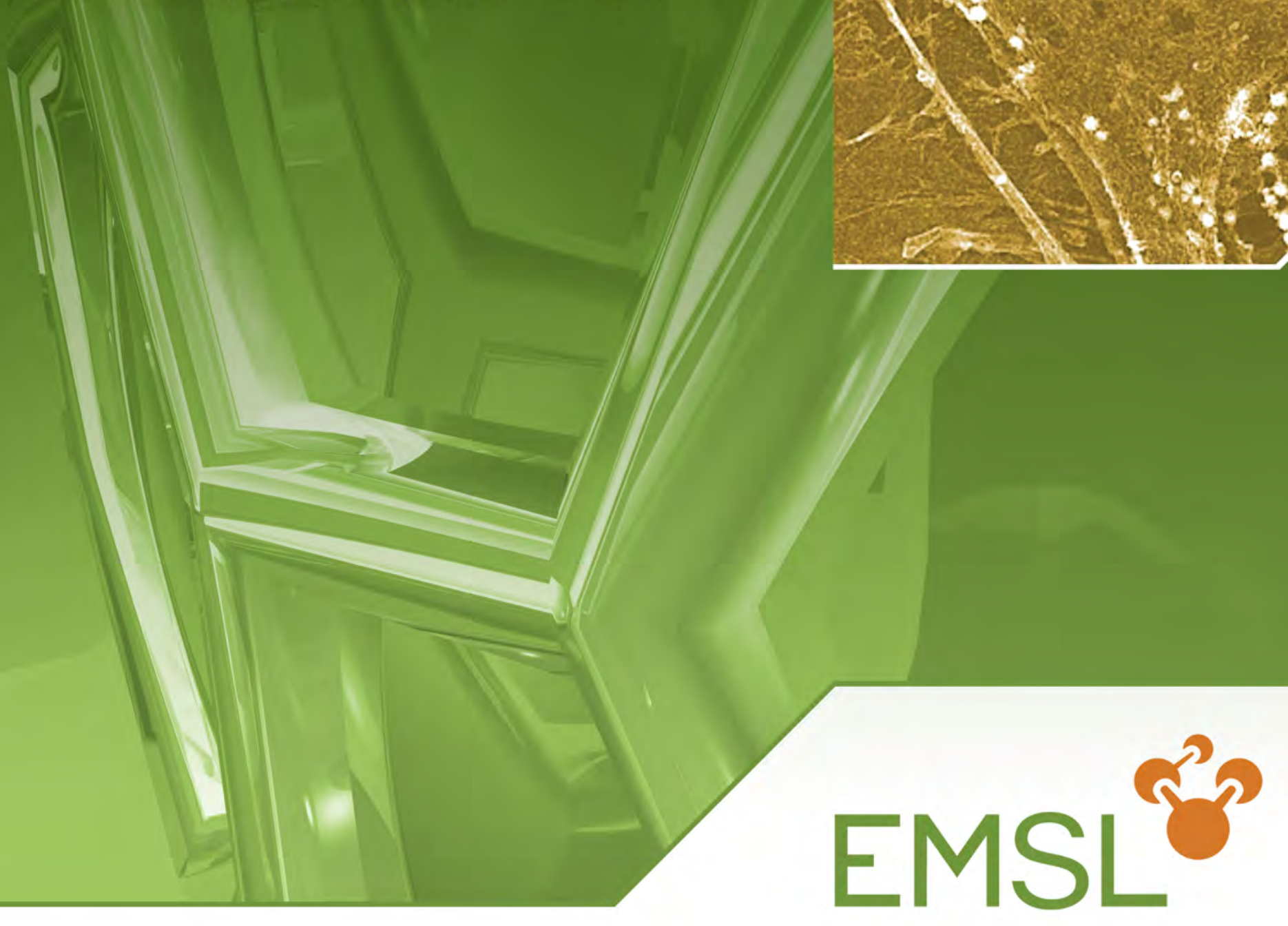




\title{
DISCLAIMER
}

This report was prepared as an account of work sponsored by an agency of the United States Government. Neither the United States Government nor any agency thereof, nor Battelle Memorial Institute, nor any of their employees, makes any warranty, express or implied, or assumes any legal liability or responsibility for the accuracy, completeness, or usefulness of any information, apparatus, product, or process disclosed, or represents that its use would not infringe privately owned rights. Reference herein to any specific commercial product, process, or service by trade name, trademark, manufacturer, or otherwise does not necessarily constitute or imply its endorsement, recommendation, or favoring by the United States Government or any agency thereof, or Battelle Memorial Institute. The views and opinions of authors expressed herein do not necessarily state or reflect those of the United States Government or any agency thereof.

\author{
PACIFIC NORTHWEST NATIONAL LABORATORY \\ operated by \\ BATTELLE \\ for the \\ UNITED STATES DEPARTMENT OF ENERGY \\ under Contract DE-AC05-76RL01830
}

\author{
Printed in the United States of America \\ Available to DOE and DOE contractors from the \\ Office of Scientific and Technical Information, \\ P.O. Box 62, Oak Ridge, TN 37831-0062; \\ ph: (865) 576-8401 \\ fax: $(865) 576-5728$ \\ email: reports@adonis.osti.gov

\begin{abstract}
Available to the public from the National Technical Information Service, U.S. Department of Commerce, 5285 Port Royal Rd., Springfield, VA 22161 ph: (800) 553-6847 fax: (703) 605-6900

email: orders@ntis.fedworld.gov

online ordering: http://www.ntis.gov/ordering.htm
\end{abstract}





\section{Dynamic Processes in Biology, Chemistry, and Materials Science: Opportunities for UltraFast Transmission Electron Microscopy Workshop Summary Report}

June 14-15, 2011

Prepared for the U.S. Department of Energy's Office of Biological and Environmental Research under Contract DE-AC05-76RL01830

Pacific Northwest National Laboratory

Richland, Washington 



\section{Executive Summary}

This report summarizes a 2011 workshop held at the Environmental Molecular Sciences Laboratory (EMSL) in Richland, Washington, that addressed the potential role of rapid, time-resolved electron microscopy measurements in accelerating the solution of important scientific and technical problems. A series of U.S. Department of Energy (DOE) and National Academy of Science workshops have highlighted the critical role advanced research tools play in addressing scientific challenges relevant to biology, sustainable energy, and technologies that will fuel economic development without degrading our environment. Among the specific capability needs for advancing science and technology are tools that extract more detailed information in realistic environments (in situ or operando) at extreme conditions (pressure and temperature) and as a function of time (dynamic and time-dependent). One of the DOE workshops, "Future Science Needs and Opportunities for Electron Scattering: Next Generation Instrumentation and Beyond," specifically addressed the importance of electron-based characterization methods for a wide range of energy-relevant Grand Scientific Challenges. Boosted by the electron optical advancement in the last decade, a diversity of in situ capabilities already is available in many laboratories. These capabilities enable the investigations of biological and chemical processes in situ with the high spatial and spectroscopic resolution provided by transmission electron microscopy (TEM). The remaining major capability gap is the lack of time resolution. State-of-the-art in situ TEM instrumentation allows time resolution of seconds or milliseconds at best. Present capabilities are far removed from the natural time scale on which processes on a microscopic level occur which is between microseconds ( $\mu$ s) to attoseconds (as).

In an effort to address current capability gaps, EMSL, with support from FCSD, the Fundamental \& Computational Sciences Directorate at PNNL, organized an "Ultrafast Electron Microscopy Workshop," held June 14-15, 2011, with the primary goal to identify the scientific needs that could be met by creating a facility capable of a strongly improved time resolution with integrated in situ capabilities. The workshop brought together more than 40 leading scientists involved in applying and/or advancing electron microscopy to address important scientific problems of relevance to DOE's research mission. This workshop built on previous workshops ${ }^{1}$ and included three breakout sessions identifying scientific challenges in biology, biogeochemistry, catalysis, and materials science-frontier areas of fundamental science that underpin energy and environmental science - that would significantly benefit from ultrafast transmission electron microscopy. In addition, the current status of time-resolved electron microscopy was examined, and the technologies that will enable future advances in spatio-temporal resolution were identified in a fourth breakout session.

The major conclusion of the workshop was that significant scientific progress can be expected in each of the four scientific areas mentioned above by the development of an ultrafast transmission electron microscope capability (UTEM) for time-resolved, in situ measurements capable of picosecond-scale image acquisition. Such advances are critical for understanding fundamental processes in biology, biogeochemistry, chemistry, and materials science. For example, charge transfer and intermediate states, which can be characterized by the UTEM, are critical for understanding catalytic processes in biology and chemistry that could result in higher efficiency for industrial processing. High-impact applications of the UTEM will provide important insights regarding the efficient energy transduction mechanisms, including the assembly and molecular actions of supramolecular protein machines in biology. A better understanding of the mechanism of action of cellulases has the potential to develop more efficient enzymes for the enzymatic deconstruction of plant cell walls into their fermentable sugar building blocks. The UTEM will provide the spatial and temporal resolution needed to move a generation of hypotheses from in silico to in situ. Combining enzyme simulation with observation provides the data foundation upon which engineering strategies to accelerate economic production of

${ }^{1}$ http://science.energy.gov/bes/news-and-resources/reports/workshop-reports/ and http://science.energy.gov/bes/news-andresources/reports/basic-research-needs/. 
biofuels can be based. UTEM will also provide insight into interfacial electron transfer to mineral surfaces in biogeochemistry and dynamic and time varying processes in catalysis. Phonon mediated processes, also accessible for UTEM, are essential for understanding mechanical behavior as well as radiation effects in both engineering and biological materials. These processes happen on an atomic or on a nanometer scale but are embedded in larger scale processes, such as interaction between cells in microbial communities. These larger scale processes, which occur on a micrometer length scale and a microsecond timescale, are also observable with UTEM. In addition to the obvious advantages of highresolution time-resolved data collection, ultra-fast measurements will enable new types of in situ microscopy including the ability to make measurements in extreme environments and live cells. The inherent minimization of damage during time-resolved measurements is particularly important for studies of biological systems and for in situ real time measurements of chemical processes, such as catalytic reactions.

The ultrafast TEM capabilities that were discussed in the workshop build on established technologies, such as pulsed electron emitters and aberration correction. Integrating these components with present in situ TEM methods will provide a significant enhancement - possessing all the same flexibility in environmental control but with many orders of magnitude improvement in combined spatio-temporal resolution. UTEM will integrate single shot and stroboscopic modes in one instrument enabling measurements on reversible and irreversible processes over a wide range of time scales, from milliseconds to femtoseconds. All the components for UTEM, such as electron optics, pulsed electron emitter and in situ instrumentation, are now available and the time is right for DOE to consider building an integrated UTEM facility to meet new scientific challenges.

The ability of an electron microscope to provide a direct image of a structure, or direct movie of a transient event, also provides access to new areas of scientific research that cannot be reached by other ultrafast methods, such as x-ray diffraction using the latest generation light sources, where the inversion from a diffraction pattern and the smaller scattering cross-section of x-rays compared to electrons limits the types of samples that can be studied. A further benefit of the integration of spatial and temporal lenses into an optimized UTEM is that the microscope will have capabilities for imaging, diffraction, and spectroscopy that are comparable to, and in some cases exceed, the best conventional instruments that currently exist.

DOE user facilities are well equipped for complex instrumentation such as UTEM and they have a successful record in development and operation of these instruments for the benefit of science groups in the US and abroad. . EMSL is highly suited for UTEM because EMSL's experimental capabilities and scientific scope encompasses biology, biogeochemistry, chemistry, and material science, with an emphasis on integration of such capabilities to address significant, pressing problems that are facing DOE and the nation. Also EMSL's extensive experience in managing complex projects and successfully integrating these capabilities in impactful scientific outcomes makes EMSL an ideal facility to design, build and commission a UTEM capability.

In this Workshop Report, the spatial, temporal and energy resolution needed for grand challenge research in biology, biogeochemistry, catalysis, and material science is discussed, and a plan for implementing this paradigm changing capability is discussed 


\section{Acknowledgment}

The workshop was organized by:

- Bernd Kabius, EMSL, Pacific Northwest National Laboratory

- Nigel Browning, FCSD, Pacific Northwest National Laboratory

- Theva Thevuthasan, EMSL, Pacific Northwest National Laboratory

- Eric Stach, CFN, Brookhaven National Laboratory

The workshop was strongly supported by Lou Terminello, FCSD, Pacific Northwest National Laboratory

The workshop was structured around the scientific areas biology and biogeochemistry (Leadership: Mike Hochella); catalysis (Leadership: Bruce Gates); and materials science (Leadership: George Crabtree). Common sessions covered keynote talks on these topics, as well as shorter discussions on the state-of-the-art instrumentation for ultra-fast TEM. Scientific challenges were discussed in breakout session on these four topics.

The workshop agenda can be found in Appendix B.

\section{Biology and biogeochemistry}

James Evans, Don Baer, Jill Banfield, Haluk Beyenal, Daniel Bond, Edgar Buck, Luis Comolli, Nancy Hess, Mike Hochella, Scott Lea, Matt Marshall, James McKinley, Karin Rodland, Tanvir Shaikh, David Shuh, Tom Squier, Bill Shelton, Ray Teller, and Ping Yang.

\section{Catalysis}

Bruce Gates, Chongmin Wang, Amity Andersen, Ayman Karim, David King, Jingyue Liu, Karl Mueller, Chuck Peden, Warren Pickett, Robert Rioux, Renu Sharma, Stefan Vajda, Yong Wang, Niri Govind, Robert Colby, and Ken Lopata.

\section{Material Science}

SK Sundaram, Chuck Henager, Rick Kurtz, Scott Chambers, Matthew Olszta, Charudatta Phatak, Orlando Auciello, Christian Mailhiot, Warren Pickett, Ulrich Welp, Niri Govind, Vincent Meunier, Libor Kovarik, Chuck Henager, Steve Bruemmer, Danny Edwards, Shannon Goodwin, Brad Johnson, Toya Miller, Bill Shelton, and George Crabtree. 


\section{Acronyms and Abbreviations}

$\AA$

$\mu \mathrm{s}$

ACTF

AIMD

$\mathrm{Al}_{2} \mathrm{O}_{3}$

ATP

$\mathrm{BaO}$

BCC

BER

BES

$\mathrm{C}_{\mathrm{c}}$

CCD

CMOS

$\mathrm{C}_{\mathrm{s}}$

CZT

DFT

DOE

dpa

DTEM

EDXS

EELS

EFTEM

ELNES

EMSL

EXELFS

FCSD

FEG

FEL

$\mathrm{FDH}_{\mathrm{H}}$

GISAXS

GTP

HRTEM

LCLS

LLNL

MEMS

$\mathrm{meV}$
Angstrom

microsecond

amplitude contrast transfer function

$a b$ initio molecular dynamics

alumina

adenosine triphosphate

barium oxide

body centered cubic

Biological and Environmental Research

Basic Energy Science

chromatic aberration

charge-coupled device

complementary metal-oxide-semiconductor

spherical aberration

CdZnTe

density functional theory

U.S. Department of Energy

displacements per atom

dynamic transmission electron microscopy

energy dispersive x-ray spectroscopy

energy electron-loss spectroscopy

energy filtered transmission electron microscopy

electron loss near edge structure

Environmental Molecular Sciences Laboratory

extended energy loss fine structure

Fundamental \& Computational Sciences

field emission gun

free-electron laser(s)

formate dehydrogenase

grazing-incidence small-angle scattering

guanosine triphosphate

high-resolution transmission electron microscopy

Linac Coherent Light Source

Lawrence Livermore National Laboratory

micro electromechanical systems

milli electron Volt 
MGD

$\mathrm{ms}$

NFS

$\mathrm{nm}$

NMR

ns

NSR

PKA

PNNL

ps

RF

SeCys

SEM

STEM

STM

STR

TD-DFT

TEAM

TEM

UC-Davis

UTEM

XAFS

XANES molybdopterin guanidine dinucleotide(s)

millisecond

nanostructured ferritic steels

nanometer

nuclear magnetic resonance

nanosecond

$\mathrm{NO}_{x}$ storage/reduction

primary knock-on atom

Pacific Northwest National Laboratory

picosecond

radio frequency

selenocysteine

scanning electron microscopy

scanning transmission electron microscopy

scanning tunneling microscopy

spatial and temporal resolution

time-dependent density functional theory

transmission electron aberration-corrected microscope

transmission electron microscopy

University of California, Davis

Ultra-fast Transmission Electron Microscopy

$\mathrm{x}$-ray absorption fine structure

$\mathrm{x}$-ray absorption near edge structure 


\section{Units of measurement}

$\begin{array}{ll}\mathrm{ms} & \begin{array}{l}\text { millisecond } \\ \text { microsecond } \\ \text { nanosecond } \\ \mathrm{ns}\end{array} \\ \mathrm{ps} & \text { picosecond } \\ \mathrm{fs} & \text { femtosecond } \\ \mathrm{nm} & \text { nanometer } \\ \AA & \text { Angstrom } \\ \mathrm{eV} & \text { electron volt } \\ \mathrm{meV} & \text { millielectron Volt } \\ \mathrm{K} & \text { Kelvin }\end{array}$




\section{Contents}

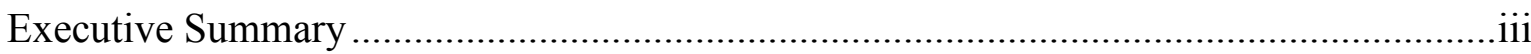

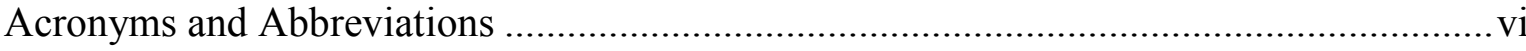

Units of measurement .............................................................................................ii

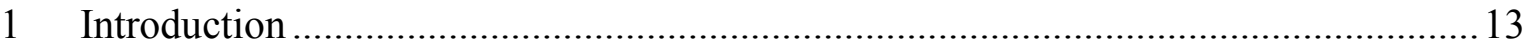

1.1 Scientific Challenges of Ultra-fast Processes for Energy Science ........................ 13

1.2 Present Capabilities of TEM, DTEM and UTEM Concepts ……………...............17

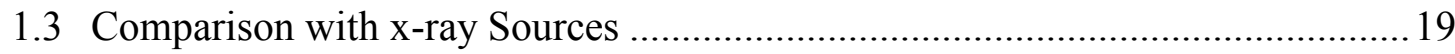

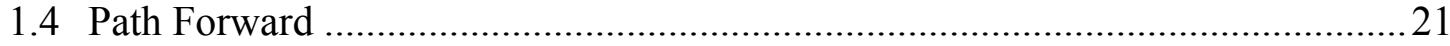

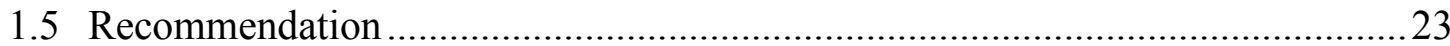

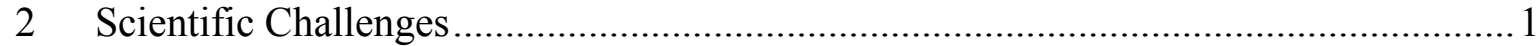

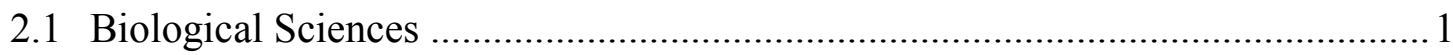

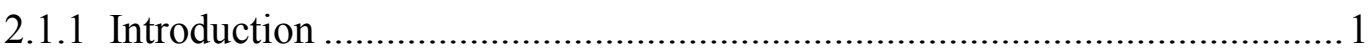

2.1.2 Limitations of Available In Situ Biology Capabilities .................................... 3

2.1.3 Natural Community Systems .................................................................. 4

2.1.4 Observations of Living Tissue..................................................................

2.1.5 Importance of UTEM Imaging to Biology ………………………………....

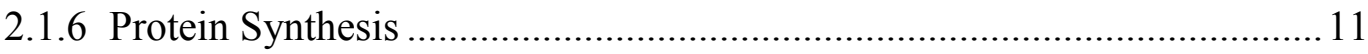

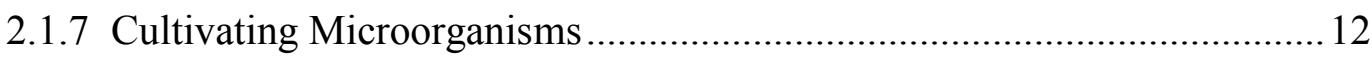

2.2 Applications of Ultrafast Electron Microscopy in Biology and

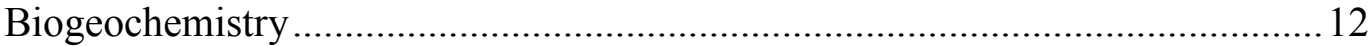

2.2.1 Biotransformation of Actinides or other Multivalent Metals ....................... 13

2.2.2 Formation of Clusters, Nanoparticles, and Colloids .................................... 14

2.2.3 Structure, Reactivity, and Distribution of Environmental Nanoparticles:

From Aerosols to the Smallest Colloids to Confined Fluids ........................ 16

2.2.4 Transport of Nanoparticles ......................................................................... 17

2.2.5 Proposed Applications of UTEM at the Interface of Biology and

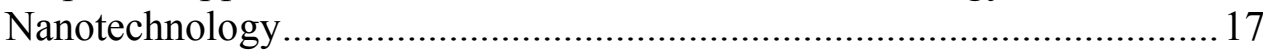

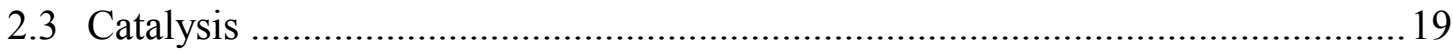

2.3.1 Introduction: TEM and Catalysis ........................................................... 19

2.3.2 Images of Catalytic Species on Surfaces under Static Conditions .............. 19

2.3.3 How UTEM will Affect the Understanding of Catalysis ............................22 
2.3.4 Time Scales Characteristic of Catalytic Processes ...................................223

2.3.5 The Dynamic Nature of Catalytic Nanostructures and Catalytic Sites ...... 24

2.3.6 Spectroscopy of Catalysts Used in Conjunction with TEM.....................227

2.3.7 Statistical Assessment of TEM Data and the Motivation for Investigation of Well-defined Model Catalysts........................................ 30

2.3.8 Additional Challenges and Opportunities .............................................. 32

2.3.9 Connecting Experimental Results with Theory..................................... 33

2.3.10Observation of Highly Fluxional Rhodium Catalysts for the Dehydration of Amino Boranes............................................................ 34

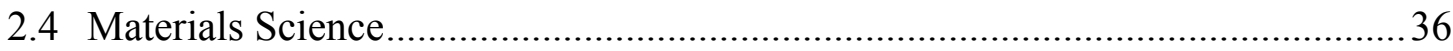

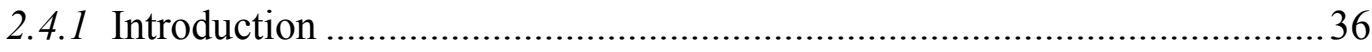

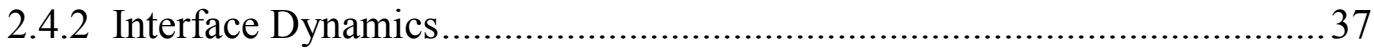

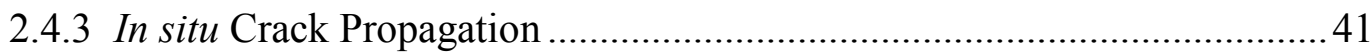

2.4.4 Polarization Dynamics in Ferroelectric and Multiferroic

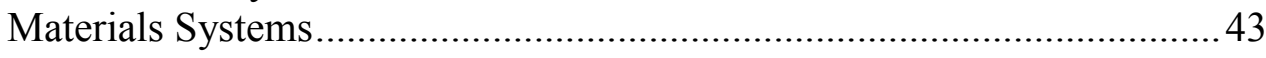

2.4.5 Resistive Switching Memory Materials ................................................ 45

2.4.6 Phase Transitions and Fluctuations ...................................................... 47

2.4.7 Fluctuations in Correlated Electron Materials: a "Killer App" for Ultrafast Electron Microscopy ......................................................... 49

2.4.8 Strongly Correlated Electrons and UTEM ..........................................5 50

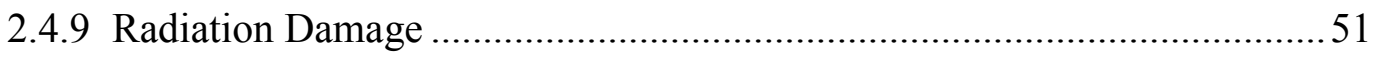

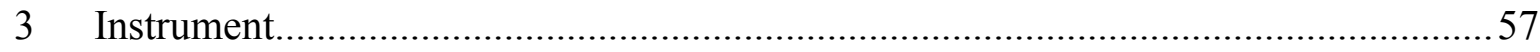

3.1 Design and Implementation of an Ultrafast Transmission Electron Microscope. 57

3.2 Obtaining Temporal Resolution in TEM with a Photo-Emission Source .............57

3.3 Two Contrasting Modes of Operation: Stroboscopic and Single Shot.................58

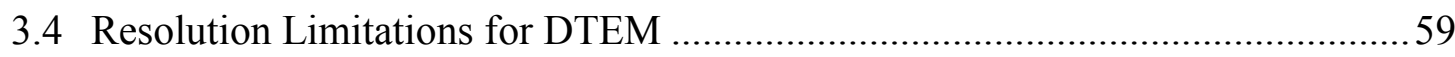

3.5 The Evolution from DTEM to a True Single-Shot UTEM ................................62

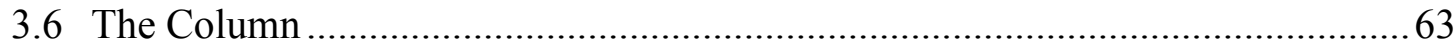

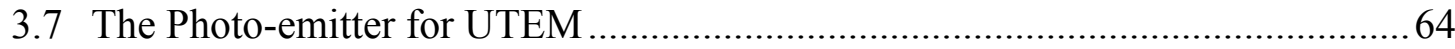

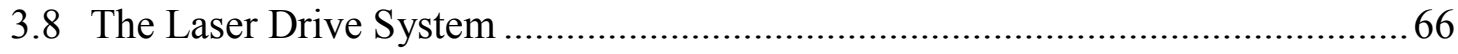

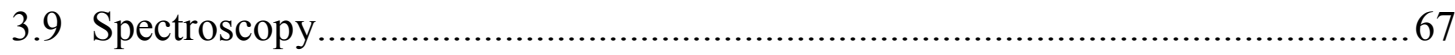

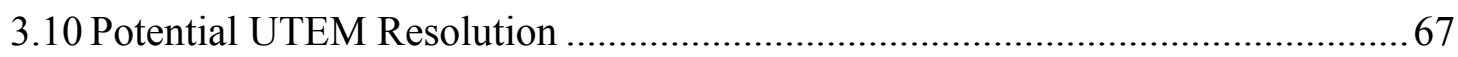

3.11 The Significance of Single-shot UTEM in the Global Research Community .....69

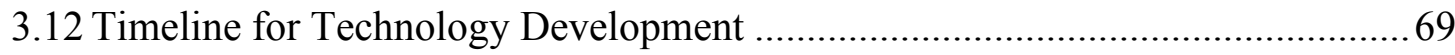




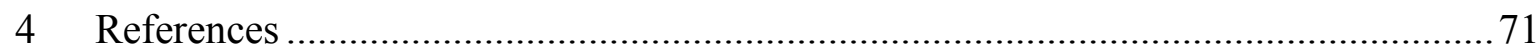

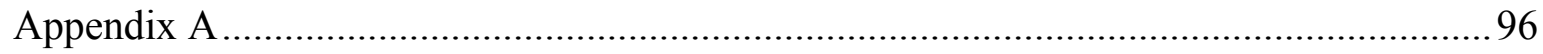

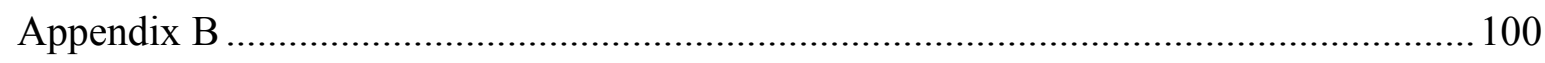

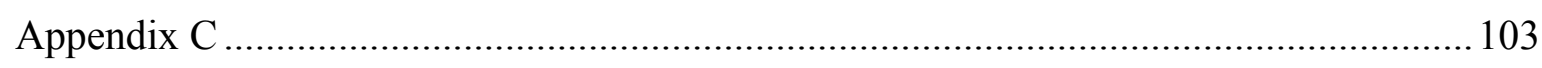




\section{Tables}

Table 1............................................................... Timeline for the construction of a UTEM. 70 


\section{Introduction}

\subsection{Scientific Challenges of Ultra-fast Processes for Energy Science}

The 20th century saw dramatic progress in observing and understanding the behavior of organic and inorganic matter in terms of their constituent atoms and electrons. Spurred by remarkable advances in experimental resolution of space, time, and energy and the intellectual leaps and descriptive power of quantum mechanics, atoms and electrons emerged from abstract organizational concepts at the beginning of the last century to practical building blocks that can be observed, assembled, and manipulated in a multitude of ways. However, most of humankind's knowledge of atoms and electrons is in the equilibrium or near-equilibrium limit, where behavior changes slowly with time. Although it is known that chemical reactions are inherently dynamic and have been examined using optical methods for decades, probing and understanding the dynamics of biological, chemical, and solid-state processes that account for most of their functionality is in its early stages. Bond breaking and making; the transfer of energy among electronic, lattice, and magnetic degrees of freedom; and the fluctuations that promote phase transformations happen on ultrafast time scales - picosecond or shorter - and far from equilibrium. They remain largely beyond our experimental and theoretical reach, particularly for methods that have the highest spatial resolution.

The crucial importance for high time and spatial resolution under in situ condition is indicated by frequent calls for additional dynamic and time resolved measurements in U.S. Department of Energy (DOE) and related National Academy workshop reports. These calls and reports cover a wide range of topics from instrumentation ${ }^{1}$ to addressing BER specific scientific topics relevant to biology ${ }^{2,3}$, biogeochemistry ${ }^{4}$, catalysis, carbon capture, general DOE related topics such as compact light sources, electron scattering, solid state lighting and energy storage ${ }^{5}$. Although geological and biological processes have time scales of days, months, and years, the molecular- and atomic-scale mechanisms, such as protein folding and electron transfer processes that influence these longer scale behaviors, occur much more quickly. Therefore, the dynamics of processes that impact subsurface contaminant transport ${ }^{2,6}$, carbon capture and storage, as well as other aspects of biology, cover a large time scale and will benefit from rapid time resolved and in situ ${ }^{1}$ measurements. The National Academy of Sciences report ${ }^{1}$ on advanced instrumentation and facilities frequently noted the importance of dynamical measurements and specifically called out the need for dynamic electron microscopy. Not only will timeresolved measurements advance the ability to measure important processes, they will both stimulate new theoretical work and provide the basis for testing and verifying these theoretical efforts. Observing dynamic behavior across space and time scales is an essential first step in explaining and ultimately controlling far-from-equilibrium phenomena and functionality.

The natural time scale of dynamic processes depends on the area of interest, from attoseconds to femtoseconds for electronic transitions; femtoseconds to picoseconds for atomic, molecular, and lattice dynamics; picoseconds to nanoseconds for magnetic and ferroelectric polarization reversal; and from nanoseconds to microseconds for atomic diffusion processes involved in nucleation and growth phenomena. The natural length scale for dynamic processes ranges from the atomic and molecular scale, $0.1-10 \mathrm{~nm}$, up to the scale of interactions between biological cells and grains in metallic alloys.

The dynamic responses of materials typically strongly interact with each other-electronic, structural, and spin degrees of freedom cooperate in chemical reactions, phase transitions, and polarization reversals. This interaction dramatically affects the time scale of a given phenomenon. For example, in bond-breaking chemical reactions, photoexcitation of a diatomic molecule to an excited electronic state may require only attoseconds, but the subsequent separation of the molecule as it relaxes toward dissociation requires several vibrational cycles on the picosecond time scale. For more 
complex chemical reactions requiring bond breaking and bond forming, the time scale can be longer to accommodate the more complex atomic rearrangement. The strong interaction between electronic, structural, and magnetic degrees of freedom effectively slows down fast electronic processes and provides additional experimental signatures of the composite dynamics. Full characterization of the time sequence and interaction of the dynamic responses requires integrating information from separate probes of each degree of freedom. Figure 1 offers an overview of the time and length scales of dynamic process in the major scientific topics - biology and biogeochemistry, catalysis, and materials science - on which this workshop was focused and where a project can access each scientific challenge.

\section{Opportunities for capability expansion through UTEM in the time/space domain: dynamic processes in Biology, Chemistry and Materials Science}

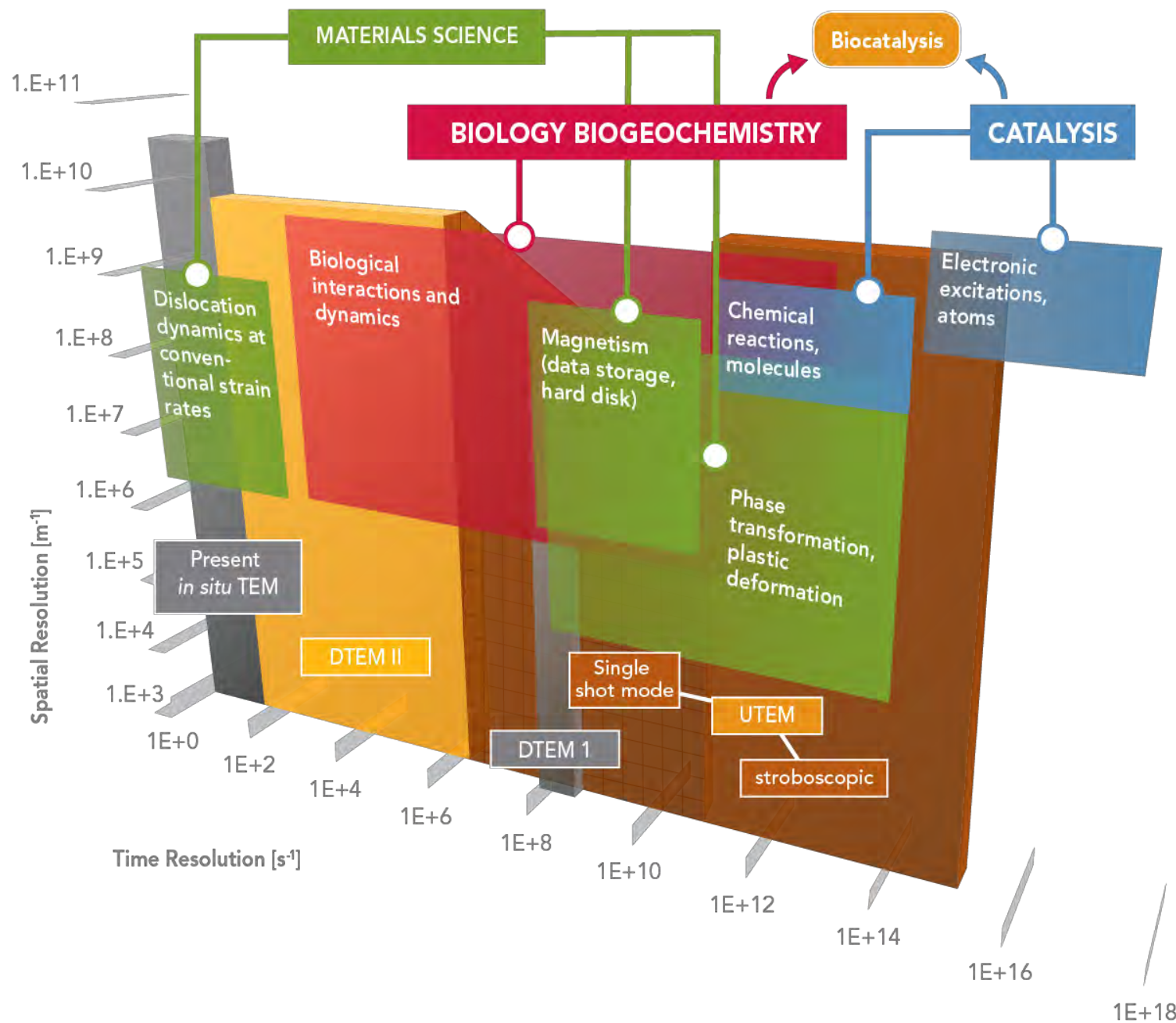

Figure 1. Scientific challenges, dynamic process, and their intrinsic time and length scales. The cuboids in the background (present in situ TEM, DTEM I, DTEM II, and UTEM) indicate which technology must be used to access certain processes. DTEM I is the dynamic transmission electron microscope (DTEM I) at Lawrence Livermore National Laboratory (LLNL). DTEM II development was started at the University of California, Davis (UC-Davis) and is currently being transferred to PNNL. The 
proposed, new UTEM capability will build on prior projects and will enable observation of shorter time scale processes that are beyond the reach of current capability.

While the need for dynamic information applies to most areas of science, the workshop reported here focused on electron microscopy and the science need in three specific areas. In each area, several topics were identified for which ultrafast transmission electron microscopy could enhance scientific discovery:

\section{Biological Sciences and Biogeochemistry}

Cellular responses to changes in their environment, such as microbial communities or multicellular eukaryotic organisms, are one of the great challenges in biological sciences. These responses include complex dynamic processes involving synthesis, assembly, and turnover of the cellular machinery. An integrated approach including proteomics, structural biology, and UTEM will advance our understanding of cellular response to changing environmental signals. Critical to these goals are the high time and spatial resolution of the UTEM concept. Such measurements offer an ability to validate biological function and facilitate the interoperability of biological modules and processes in both lab based and environmental systems.

UTEM is expected to permit measurement of high-resolution structures of individual proteins, macromolecular complexes, organoids, and viruses in solution without the need for crystallization. These new methods may permit "diffract and destroy" imaging where the diffraction pattern is collected for individual molecules prior to the onset of radiation induced damage or motion. Pulsed beam operation also minimizes the electron dose because the sample is only exposed to the electron beam for data acquisition. Ultimately, it is anticipated that these new methods will enable the creation of movies of protein conformational changes related to catalysis, as well as the identification of macromolecular structures in viruses or intact living microbes. UTEM allows access to the time and length scale of molecular machines permitting measurements of assembly and functional dynamics.

Several important roles have been identified for UTEM including: nanoparticle nucleation and transport, imaging of living tissue, determining the structure of individual proteins and protein dynamics, measurements of functional dynamics of molecular machines, and examination of diversity in microbial communities.

Geochemical and biogeochemical processes play major roles in the Earth's chemical cycles and important processes including contaminant transport, bioremediation, biocatalysis, $\mathrm{CO}_{2}$ sequestration and bioenergy production. Understanding these systems requires the unique spatial/time resolution of UTEM for the aforementioned biological processes while interacting with a geological environment (pressure, temperature, inorganic compounds).

\section{Catalysis}

Catalysts are critical components of many current technologies and essential components of sustainable energy systems ranging from fuel cells and batteries to turning biomass into useful chemicals or fuels. Catalysis involves many dynamic and time-varying processes, of which many can be examined by UTEM approaches. Relevant times for catalytically important processes range from femtoseconds to hundreds of seconds. It is particularly relevant to understand and visualize transient processes, such as structural changes during catalysis operation, substrate catalyst interactions, and intermediate states. In addition, the application of UTEM methods can serve to minimize any perturbing impacts of the electron beam in situ studies. The types of detailed information that can be available from UTEM should facilitate advancing theoretical understanding and design of next-generation catalysts by allowing theory, characterization and function to be integrated. The development of UTEM will both require and enable a new type of pump-probe experiment, not generally applied to catalysis work, to be developed and exploited. 


\section{Materials Science}

Materials science has been a traditional domain of electron microscopy. Historically, the structures of a material and material interfaces have been considered in a mostly static manner, with small fluctuations around that static structure being considered. Increasingly, it has been recognized that dynamic processes determine many materials and interfacial properties, ranging from material cracking to photoexcitation processes. Dynamic processes identified as important for UTEM study by workshop participants included the solid-liquid interface, crack propagation, resistive switching in memory materials, phase transitions, fluctuations in correlated electron materials, radiation damage, and structures (including defects) of nanomaterials.

\section{Microscopy Opportunities and Challenges}

As the world embraces and makes more routine use of the exceptionally high spatial resolution afforded by the new breed of aberration-corrected electron microscopes, there remain two exciting frontiers in electron imaging: 1) in situ measurements and 2) imaging in very short time domains. The former capability (in situ measurements) is a simple extension of current electron microscopy technology and currently is being developed by a number of commercial partners (such as Hummingbird Scientific, FEI, and JEOL). Conversely, the latter capability (short time frame microscopy) is equally exciting but will require new electron sources to reach the picosecond timescales that allow new insights into irreversible materials and biological phenomena of interest to DOE.

\section{Scientific Impact of Electron Microscopy and the Challenge of Ultrafast Measurements}

Electron microscopy provides probes of electronic, structural, and polarization degrees of freedom at high spatial resolution. Spherical and chromatic aberration correction allows unmatched focusing to angstrom $(\AA)$ and sub- $\AA$ spatial resolution for imaging the structure and motion of isolated nonperiodic objects, such as atoms, molecules, defects, interfaces, and amorphous structures. Electron diffraction allows detailed structural characterization of periodic systems using much lower intensity electron beams. Electron energy loss spectroscopy (EELS) probes the electronic excited states of materials with atomic resolution, and Lorentz imaging and holography probe magnetic states with nm resolution. Collectively, these electron probes cover most phenomena in materials dynamics. Often, these probes can be applied to the same sample in the same experimental apparatus, avoiding complications due to sample variation. Electron interaction with matter is much stronger than that of x-rays, making an electron beam probe much more efficient for analyzing small volumes.

The challenge in applying electron microscopic imaging to dynamic molecular and atomic processes is achieving electron pulses of sufficiently high intensity and short duration to freeze the electronic, structural, or chemical motion. Remarkable progress in producing short pulses by laser-induced photoemission has allowed the time resolution of electron imaging to be pushed through the microsecond to the nanosecond regimes. ${ }^{7-9}$ Progress in ultrafast lasers and photocathode materials now brings the prospect of UTEM at picosecond time scales well within reach, opening new time regimes for probing ultrafast dynamic behavior. Stroboscopic illumination already has achieved femtosecond resolution ${ }^{10}$, a major advance for systems that are perfectly reversible for millions of repetitions. One of the Grand Challenges in ultrafast electron microscopy is achieving comparable time resolutions for processes that are not perfectly reversible. In this report, we explore the new scientific horizons that ultrafast electron microscopy opens for capturing the dynamics of materials at picosecond and faster times scales in three broad areas: biogeochemistry, catalysis, and material science. 


\subsection{Present Capabilities of TEM, DTEM and UTEM Concepts}

During the last two decades, electron microscopy has witnessed an unprecedented number of innovations that enhanced existing approaches and introduced qualitatively new techniques. One of the most important novel technologies is aberration correction. Correction of spherical aberration $\mathrm{C}_{\mathrm{s}}$ has been demonstrated by Haider et al. ${ }^{11}$ In the years that followed, this hexapole corrector was applied to various materials science problems ${ }^{12-14}$ and is now a standard for highresolution TEM (HRTEM). A quadrupole/octopole corrector was developed and applied by Dellby et al., ${ }^{15}$ for scanning transmission electron microscopy (STEM) mode. Since then, aberration correction has been used to help solve diverse material science problems. ${ }^{16-18}$ Recently, correction of chromatic aberration has been demonstrated ${ }^{19}$ and successfully applied to improve resolution in energy-filtered TEM (EFTEM) by a factor of five. Furthermore, there has been significant progress in EELS, resulting in improved energy resolution (monochromator) and a larger field of view for electron-spectroscopic imaging ${ }^{20}$ and energy-filtered imaging. ${ }^{21}$ Micro electromechanical systems (MEMS) technology have also been employed to fit experimental equipment into the TEM. ${ }^{22}$

All of these new developments have benefitted in situ TEM by allowing more space for experimental equipment around the sample, achieving better resolution in gaseous or liquid environments ${ }^{23}$ and thereby stimulating a significant increase in the number of publications in this field. A further indication for this trend is the diverse variety of in situ sample holders, which now are available and/or under development.

Many of the requirements for the investigation of dynamic processes by in situ TEM already are in place: spatial resolution, environment, and analytical electron microscopy and in situ instrumentation for TEM. While research has made these static requirements for ultrafast TEM readily available, the fundamental temporal resolution remains severely lacking. Resolving picosecond or faster dynamic process on a nanometer or an atomic scale requires short intense electron pulses - a frontier now poised for rapid progress. Dynamic processes on a nanometer or an atomic scale are occurring on a time scale between nanoseconds and attoseconds, while most in situ experiments to date offer a time resolution up to 30 frames per second with a spatial resolution of about $0.2 \mathrm{~nm}^{24}$. In characterizing a TEM for observing dynamic processes, it is useful to define the product of spatial and temporal resolution (STR) for this instrument: $3 * 10^{-2} \mathrm{~s} * 10^{-10} \mathrm{~m}=3 * 10^{-2} \mathrm{~m} * \mathrm{~s}$.

\section{Examples demonstrating the need for UTEM}

The enzymatic deconstruction of plant cell walls into their fermentable sugar building blocks is highly relevant to BER's bioenergy mission. Enzyme costs are a key cost bottleneck for production of lignocellulosic biofuels. To design more efficient enzymes, we need to better understand the mechanism of action of cellulases. Currently, computational simulations have been performed that lead to hypotheses about how these important enzymes interact with and catalyze deconstruction of their substrates (see Figure $2^{25}$ ). The UTEM will provide the spatial and temporal resolution needed to move a generation of hypotheses from in silico to in situ. Combining enzyme simulation with observation provides the data foundation upon which engineering strategies to accelerate economic production of biofuels can be based. 


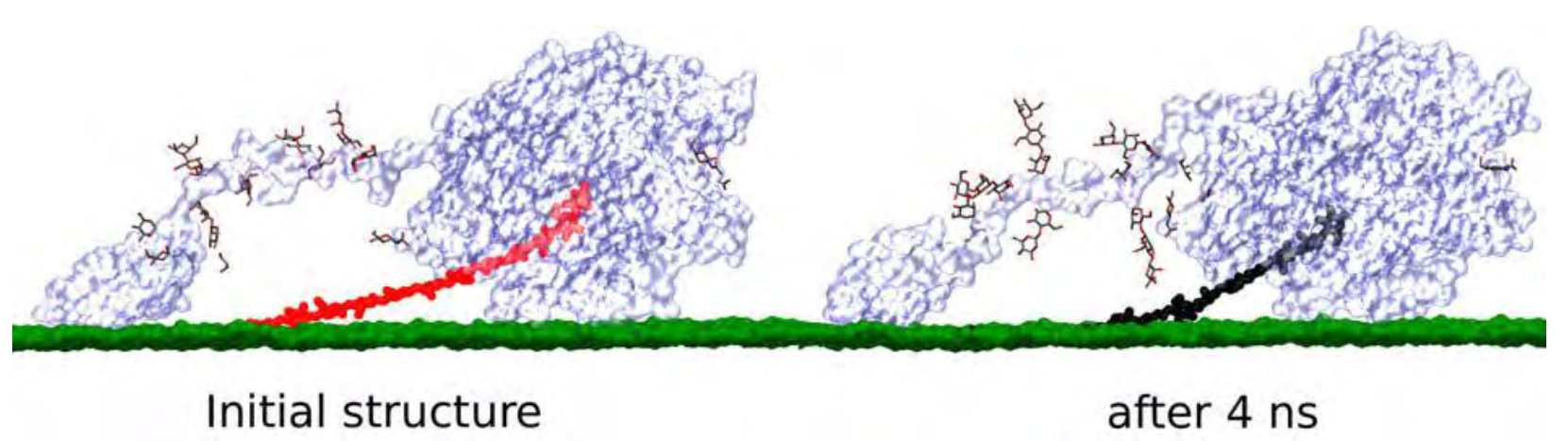

Figure 2. The initial conformation (left) of the substrate chain (shown in red); (at right) the final conformation (shown in black). ${ }^{25}$ This is a modeled depiction, but the UTEM approach would provide actual, dynamic conformational detail of this process at the nanosecond timescale.

A material science example demonstrating the limitation of the present approach can be seen in Figure 3, which shows two consecutive frames taken from a movie of grain boundary movement in $\mathrm{Au} .{ }^{26}$ The grain boundary moves during the time between these frames $\left(\sim 3 * 10^{-2} \mathrm{~s}\right)$ by approximately two nanometers. This process involves thousands of atoms moving and reconnecting to the lattice of the adjacent grain. Present instrumentation shows only the rapid movement of the grain boundary, but delivers no insight into the mechanism of grain boundary movement and intermediate steps.
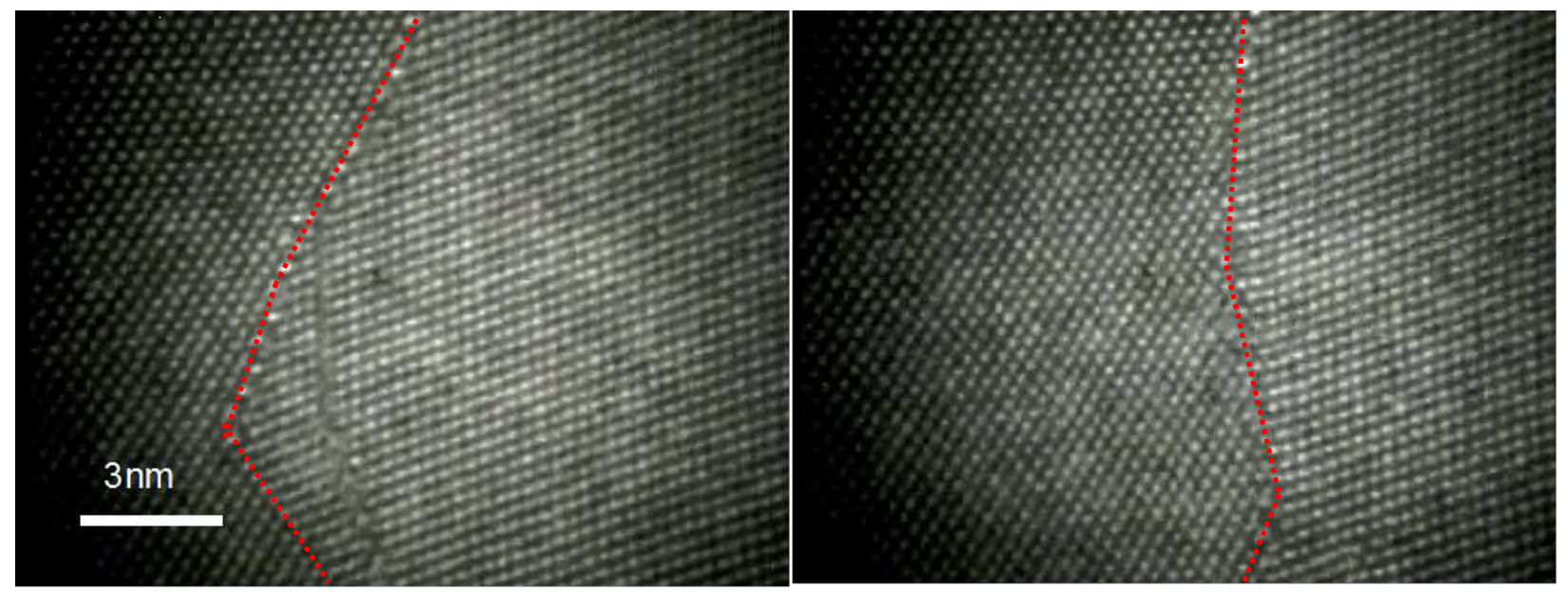

Figure 3. Grain boundary movement in Au: These high-resolution TEM images show that a grain boundary (marked by the dotted red line) moves several nanometers between two frames of the movie recorded under in situ conditions. ${ }^{26}$ The details of the mechanism of grain boundary movement are beyond the time resolution of this experiment, but could be observed using the proposed UTEM approach.

This time resolution can be improved to a certain extent by charge-coupled device (CCD) cameras with faster frame rates. A direct complementary metal-oxide semiconductor (CMOS) camera, developed for the transmission electron aberrationcorrected microscope (TEAM) project, demonstrated atomic resolution with a frame rate of $400 \mathrm{~Hz}{ }^{27}$ This approach is finally limited by the electron emission rate of the electron source. If we assume a current of $100 \mathrm{nA}$ for a standard Schottky field emitter, an image size of $512^{2}$, and $\sim 100$ electrons per pixel to obtain a reasonable image quality, a time resolution of $100 \mu \mathrm{s}$ can be achieved. By accepting noisier images or increasing the electron beam current, this limit may 
be extended by one or two orders of magnitude. However, this approach s not suited for achieving the time resolution required for the scientific challenges summarized in this report.

Further improvement in time resolution requires an electron source that can deliver an electron beam current orders of magnitide higher than the current that can be extracted from present field emission cathodes. Photoemission has demonstrated that it can meet this requirement ${ }^{28}$. A detailed description of electron emitters based on photoemission can be found in Section 3.2.

There are two approaches that enable a time resolution in the range of nanoseconds to femtoseconds:

- Stroboscopic: Each pulse contains only one (or a few) electron(s). This method which has been termed "4D electron microscopy," has demonstrated femtosecond resolution ${ }^{10}$ but can only be applied to processes that are completely reversible. Although the STR in this case is $10^{-15} \mathrm{~s} * 10^{-10} \mathrm{~m}=10^{-25} \mathrm{~m} * \mathrm{~s}$ it requires $\sim 10^{8}$ cycles to obtain an image.

- Single shot: Each pulse contains a large number of electrons sufficient for a wide field of view image. Bostanjoglo and co-workers invented this approach and Lawrence Livermore National laboratory (LLNL) and UC-Davis have used it in recent years to develop a dynamic transmission electron microscope (DTEM) for both reversible and irreversible processes. ${ }^{28}$ The recent DTEM project at LLNL made a major advance in achieving a combined STR of $10^{-8} \mathrm{~s} * 10^{-8} \mathrm{~m}=10^{-16} \mathrm{~m} * \mathrm{~s} .{ }^{29,30}$ A second DTEM project at UC-Davis (the microscope will soon be relocated to EMSL) aims for an STR of $10^{-16} \mathrm{~m}^{*} \mathrm{~s}$ although in this case the temporal and spatial resolution aimed at a different set of parameters $\left(10^{-6} \mathrm{~s} * 10^{-10} \mathrm{~m}\right)$.

As it allows a broad scope of systems to be investigated, a combined instrument that affords both the single shot and the stroboscopic approaches appears to be the best choice for a DOE facility. The availability of both modes is important because with present technology femtosecond resolution can only be achieved in stroboscopic mode, while single-shot mode allows access to irreversible processes at nanosecond resolution. The UTEM would push the limit of time resolution in single-shot mode to picoseconds. A TEM with both capabilities offers access to intermediate states of chemical reactions on an atomic scale as well as to protein folding and cell interactions on a nanometer or micrometer scale, respectively. A detailed description of electron source technology for ultra-fast TEMs is offered in Sections 3.2 and 3.3. Aberration-correction technology developed during the DOE project TEAM will be used for UTEM to improve spatial resolution (Section 3.10).

\subsection{Comparison with x-ray Sources}

The recent implementation of free-electron lasers (FELs) to generate ultrafast pulses of highly brilliant x-rays has enabled pump-probe experimentation beyond the typical samples amenable to synchrotron light sources. ${ }^{31}$ For instance, only one year after coming online in 2010, the Linac Coherent Light Source (LCLS) demonstrated the first coherent x-ray diffraction from individual nanocrystals ${ }^{32}$ and large isolated viruses ${ }^{33}$. Both of these nanoscale test samples were previously undetectable on third-generation synchrotron light sources due to their size. Although the first experiments at LCLS only achieved $0.8 \mathrm{~nm}$ spatial resolution for protein nanocrystals, atomic resolution theoretically is possible with planned improvements to the beam that are already in progress. ${ }^{33}$

At first glance, it might seem as if the development of FELs on the femtosecond time scale would be the ultimate instrument for probing molecular motions and chemical transformations. However, electrons and x-rays interact with matter in fundamentally different ways offering definite advantages for using electrons over x-rays when performing atomic-scale structural analysis of thin samples ${ }^{34}$. One of the two main advantages for electrons is the versatility of electron optics and multitude of imaging and spectroscopy modes capable of being performed simultaneously on a single 
TEM for the same sample. The second advantage relates to higher atomic cross-sections when imaging with electrons in comparison to x-rays.

As shown in Figure 4, the atomic cross-sections for carbon vary as a function of wavelength (and energy) with orders of magnitude difference between x-rays and electrons. x-rays generate elastic (Thomson), inelastic (Compton) and absorption (photoelectric) scattering events via their interaction with the spatial distribution of valence electrons within a sample. ${ }^{35}$ High-energy $x$-rays $(>1022 \mathrm{MeV}$ ) also can cause pair production due to inelastic scattering. However this energy range currently is beyond the limits of FEL light sources, such as LCLS, and is therefore not considered in this discussion. For electrons, the atomic cross-sections for inelastic and elastic events are inversely proportional to the electron velocity and are roughly parallel. Because electrons are charged particles, they interact with both the positively charged nucleus and negatively charged atomic electrons and can either undergo elastic or inelastic scattering events. For both types of information sources, the elastic scattering provides phase contrast imaging and diffraction amplitudes used for structural analysis, while the inelastic events can be used for spectroscopy and Z-contrast imaging for electrons.

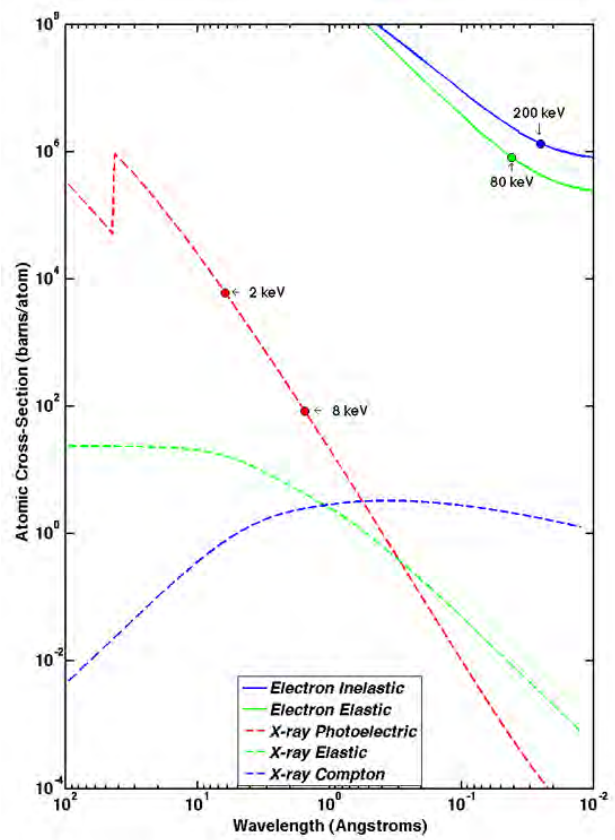

Figure 4: Comparison of atomic cross-sections (element $=$ carbon) for electrons and x-rays as a function of wavelength. Absorption, Elastic and Inelastic scattering cross-sections are displayed (red, green, and blue, respectively).

Because the energy deposited onto a sample (and by extension radiation damage) is directly proportional to the amount of inelastic scattering events by the incident electrons or x-rays, ${ }^{34}$ the more signal produced from a single elastic or inelastic scattering event the better. For x-rays, both the photoelectric and Compton cross-sections are related to inelastic scattering events. By this standard, $0.15 \mathrm{~nm}$ x-rays $(8 \mathrm{keV})$ are 1000 times worse than $200 \mathrm{keV}$ electrons $(0.0025 \mathrm{~nm})$. It should be noted that the wavelength of accelerated electrons generally is much smaller than the wavelength of x-rays used in synchrotron and FEL light sources. One accessory benefit to the smaller wavelength is an increase in the radius of the Ewald sphere for diffraction experiments that allows more faithful imaging of the two-dimensional distribution of reciprocal lattice points for thin samples.

For x-ray microscopy, the most favorable imaging conditions occur in the water window between the carbon and oxygen $\mathrm{K}$-absorption edges $\left(4.4 \mathrm{~nm}\right.$ and $2.3 \mathrm{~nm}$, respectively ${ }^{35}$ ). Within this region, the absorption by carbon-containing 
molecules (such as proteins or polymers) is approximately ten-fold greater than the absorption by an equivalent volume of water. For $200 \mathrm{keV}$ electrons, the ratio for phase contrast microscopy is only about 0.4 , suggesting an almost 25 -fold advantage for using $\mathrm{x}$-rays rather than electrons. ${ }^{34}$ However, for most chemical reactions or molecular motions, the magnitude of change will be on the sub-nanometer scale, and the best spatial resolution within the water window $(2.3 \mathrm{~nm})$ will be insufficient to observe the process. Thus, the advantage for imaging shifts back to using electrons as the information source.

In addition to the improved ratios of elastic scattering events for electrons in comparison to x-rays, the amount of energy deposited per inelastic scattering event is less for electrons than x-rays. An electron accelerated to $200 \mathrm{keV}$ deposits $20 \mathrm{eV}$ per inelastic event, whereas $8-\mathrm{keV} \mathrm{x}$-rays deposit $8 \mathrm{keV}$ of energy per inelastic event. Considering that the ratio for inelastic to elastic events is 3 for $200-\mathrm{keV}$ electrons and 10 for $8 \mathrm{keV}$ x-rays, ${ }^{34}$ the amount of energy deposited per elastic event is approximately more than 1000 times less for electrons than x-rays. Therefore, for the same radiation dose and damage threshold, electrons have a distinct advantage at wavelengths that permit atomic resolution for thin samples $(<$ $500 \mathrm{~nm})$. The same is not true for thicker specimens $(>500 \mathrm{~nm})$, where the balance tilts back in favor of x-rays since the mean free path for elastic scattering of electrons is well below the sample thickness, and too many multiple scattering events occur for reliable phase contrast imaging with electrons.

Interestingly, the recent experiments with femtosecond x-ray pulses demonstrated the feasibility of a novel "diffract-thendestroy" imaging methodology that bypasses conventional damage mechanisms caused by high flux x-ray radiation such as Coulombic explosion of molecules. ${ }^{36}$ A similar effect may occur for ultrafast electrons. However, it has not yet been detected with femtosecond electron diffraction ${ }^{37}$ or stroboscopic imaging, ${ }^{38}$ and the possibility exists to bridge the microsecond to picosecond domain for single-shot ultrafast TEM. In the meantime, the detrimental imaging effects caused by sample interaction with the electron beam, including knock-on damage, radiolysis, and hydrogen gas evolution, are anticipated to be outrun by nanosecond and picosecond electron pulses. ${ }^{38}$ In addition, for in situ liquid experiments of particles suspended in solution, the blurring effects currently caused by Brownian Motion and fluid flow will be mitigated by ultrafast pulses of better than 1 microsecond as the magnitude of movement during such a pulse will be on the picometer to angstrom scale well below the $0.1-1 \mathrm{~nm}$ spatial resolution anticipated for the UTEM.

Combined, the shorter wavelengths, higher scattering cross-sections, tunable beam, and pulse parameters for electrons permit enhanced imaging conditions for many types of samples, spanning materials science and biology, in which locality is important. The development of UTEM with pulse durations from the microsecond to picosecond time scale would enable further optimization of experimental conditions to match either the spatial and/or temporal resolution to the dynamic process of interest.

\subsection{Path Forward}

The three scientific area working groups indicated that ultrafast electron microscopy would play a major role in each area. In comparison to other methods including rapid optical measurements and those enabled by a new generation of synchrotron radiation, UTEM occupies an important and unique territory for obtaining the types of high spatial and high time resolution information needed to advances several areas of science. UTEM is particularly useful in investigating the dynamics of processes, particularly those far from equilibrium and in extreme environments, which are inaccessible to present capabilities. This includes identifying intermediate states that occur during complex processes before a system reaches equilibrium. Understanding these intermediate states is crucial for many energy-related topics, such as catalysis, biocatalysis and biogeochemistry, where these states are used to circumvent high-energy thresholds. According to theoretical models, many material-science related processes, such as radiation damage, plastic deformation, and domain reversal, include intermediate states whose experimental tests are beyond present capabilities. The time scale reaches from 
nanoseconds to attoseconds for reaction steps happening on a molecular or atomic length scale. UTEM has the capability to access the range down to picoseconds in single-shot mode and will use a stroboscopic approach for femtosecond processes. This enables UTEM to improve our understanding of fast processes in a variety of scientific topics. This report focuses on contemporary energy-related challenges in key areas, including biology and biogeochemistry, catalysis, and material science (Section 2).

During the last decade, many components, such as aberration correctors, high brightness guns, environmental cells, and energy monochromators, have been developed to further advance TEM capabilities. Novel designs for sample holders also were introduced to enable experimental capabilities inside a TEM. These developments are improving the capabilities of TEM for in situ experiments. Therefore, the major topic for TEM during the next decade will be in situ experiments. ${ }^{39}$ However, there is one major capability gap for in situ experiments: and that is time resolution. For most published in situ TEM imaging experiments, the time limit is video frame rate. The two exceptions are the femtosecond 4D UEM at the California Institute of Technology, which operates in stroboscopic mode, and the single-shot operation TEM at LLNL that is capable of 20-ns electron pulses. Both instruments are limited to either perfectly reversible processes or to a very narrow time scale, and they offer only a few types of in situ experiment capabilities.

The UTEM concept integrates the time resolution of these pump-probe instruments with state-of-the-art aberration correction technology, chemical analysis and the in situ capabilities mentioned above, thereby offering ground breaking time and spatial resolution for a large variety of in situ experiments. UTEM also offers a way to observe and understand structure and chemistry of radiation-sensitive systems, which are typical in biology and catalysis. For all systems where electron diffraction is faster than radiation damage, it is possible to avoid artifacts from the electron beam-sample interaction, such as amorphization and triggering or influencing chemical reactions by the electron beam.

Short laser pulses (or other trigger mechanisms such as electric fields) can provide extreme environments (temperature, pressure, high electric/magnetic fields) for a short time span. Only UTEM will be fast enough to image and analyze the system in this extreme environment. This capability will be important for scientific challenges in biogeochemistry and materials science.

The increasingly recognized need to understand time dependent processes, ranging from the second to faster than the femtosecond scale, requires development of new capabilities that can obtain time dependent information with high energy and spatial resolution. Although advanced x-ray methods provide important dynamical information, the physical characteristics of a UTEM system would allow it to provide information for many types of samples not available from other methods. Among its other strengths, observing dynamic behavior on its natural space and time scales via UTEM is an important step in explaining and ultimately controlling far-from-equilibrium phenomena and functionality.

A next-generation, ultrafast (herein defined as sub-nanosecond time scales) electron microscopy capability will would close the capability gap identified above, significantly enhancing overall electron microscopy analytical and characterization performance in terms of short duration chemical transformations and other previously intractable applications, such as biological systems that could not otherwise be studied because of radiation damage. Current technologies or reasonable extensions of them can be combined to create this new generation instrument, allowing dynamic low damage measurements.

The critical components for ultra-fast measurements under in situ or in operando conditions include a pulsed electron source, instrumentation for in situ TEM experiments, state-of-the-art aberration correction, and a fast and efficient detection system. Pulsed electron sources for nanosecond single shot and femtosecond stroboscopic have been demonstrated. Further development is needed to enhance the number of electrons per pulse to enhance time resolution in single shot mode to picosecond level. The consequence of high electron density in single pulses is loss of spatial and 
temporal coherency, which can be compensated by spherical and chromatic aberration correction. These correction systems have been developed and tested as part of the Transmission Electron Aberration corrected Microscope (TEAM). The alignment concepts must be adapted to the special requirements of the UTEM's pulsed electron emitter to minimize loss of spatial resolution. Direct CMOS (complementary metal-oxide-semiconductor) detectors will improve detection efficiency, which is important for UTEM because of the limited number of electrons per pulse.

\subsection{Recommendation}

The UTEM workshop defined four major scientific areas addressing DOE's mission: biology, biogeochemistry, catalysis and materials science. Especially promising opportunities were identified in bioenergy, bioconversion, and fundamental microbial processes, where time resolved information will be invaluable to understand complex processes and, in a second step, to control and improve them. Present microscopy instrumentation was found to be insufficient to address these challenges, because the natural time scale of the processes involved is orders of magnitude beyond the presently available time resolution. Present and developing technology in TEM instrumentation was deemed appropriate to close this capability gap by initiating the UTEM project, which targets the required time resolution and combines it with the in situ capabilities, high spatial and spectroscopic resolution already achieved in transmission electron microscopy. UTEM can be regarded as a critical component for the required transition of moving from static post-mortem studies to in situ investigations to understand dynamic processes. It is accordingly recommended that DOE move toward development of a UTEM capability as soon as practically possible. 



\section{Scientific Challenges}

Most of mankind's knowledge of atoms and electrons and how they transform is in the equilibrium or near-equilibrium limit, where behavior changes slowly with time. We are just beginning to probe and understand the dynamics of biological, chemical, and solid state processes that account for most of their functionality. Bond breaking and making; the transfer of energy among electronic, lattice, and magnetic degrees of freedom; and the fluctuations that promote phase transformations happen on ultrafast time scales - picosecond or shorter — and far from equilibrium. Observing dynamic behavior on its natural space and time scales by the use of ultrafast electron microscopy is an essential first step in explaining and ultimately controlling far-from-equilibrium phenomena and functionality. Developing this revolutionary capability will afford solutions to several vexing problems in areas, such as:

- Biogeochemistry: filling in gaps of knowledge of the fundamental diversity of biochemical pathways in natural systems (acknowledging that we do not know the functions of at least one-third of all proteins).

- Biochemistry and other life sciences: detailing the initiation, elongation, termination, and recycling of protein synthesis in the ribosome; imaging conformational changes in protein complexes and how living tissue responds to stimulants; and detailed knowledge of energy transduction mechanisms.

- Biocatalysis: imaging conformational changes of enzymes as a means of understanding and eventually controlling catalytic mechanisms in plants and microbial communities.

- Interfacial science: detailing the atomic complexities of molecular attachment, diffusion, crack propagation, and growth at interfaces.

- Imaging intermediates and intermediate steps in catalytic reactions, such as bond breaking and making and electron transfer in catalysis

- Making spectroscopy specific to catalytic nanostructures and accessible during imaging

- Understanding substrate-catalyst interaction

- Understanding and controlling chemical and structural changes of the catalyst under operational conditions

- Materials science: phase transitions and other dynamics, such as understanding the dynamic nature of electric polarization in ferroelectric materials, solid-solid transformations, microstructure development (nucleation and growth), fluctuations in correlated electron materials, and detailed knowledge of the mechanisms of radiation damage (and resistance thereof) in new classes of materials.

\subsection{Biological Sciences}

\subsubsection{Introduction}

A central goal of the biological sciences is to understand fundamental mechanisms involving cellular responses to environmental change, with a focus on both microbial communities and multicellular eukaryotic organisms. In all cases, cellular responses are understood to involve a range of mechanisms that typically correlate with the time-scale of the response. For example, metabolism efficiently modulates energy partitioning between different metabolic pathways using feedback mechanisms involving allosteric control mechanisms, where the product of a pathway binds to oligomeric 
regulatory proteins to adjust enzymatic function. Rapid responses to environmental change typically involve posttranslational modifications to proteins that result in shifts to metabolism involving the modulation of protein associations. Post-translational modifications involve both enzyme-mediated processes (e.g., phosphorylation, acetylation) as well as the chemical modification of sites by metabolites (e.g., oxygen) that are typically grouped as involving oxidative stress. These latter rapid responses involving post-translational modifications at sites in proteins are in contrast to long-term adjustments to metabolic pathways commonly involving transcriptional control mechanisms that adjust the concentrations of protein catalysts to allow efficient control of energy use.

Current approaches that build upon successes in genomic biology commonly focus on an analysis of transcriptional control mechanisms and their relationship to changes in protein abundance, such that a correspondence between these measurements (i.e., correlated abundance changes of transcript and protein) suggests a simple long-term regulatory strategy involving linear responses to environmental change (i.e., signal $\rightarrow$ transcriptional control $\rightarrow$ altered rates of protein synthesis). In comparison, it has proven problematic to understand nonlinear metabolic control mechanisms that mediate rapid responses to an ever-changing environment. Current approaches to understand these latter control mechanisms have been aided by the considerable strengths that permit the cataloging of proteins and their posttranslational modifications using the top-down and bottom-up proteomic capabilities, which represent major tools that are revolutionizing the biological sciences. As configured, these 'omic' methods typically are combined with a data-driven computational analysis pipeline that acts to reliably analyze a range of different samples, providing an opportunity to obtain an unprecedented identification of possible changes involving metabolic networks within cells. Coupled with emerging metabolic capabilities, it is becoming possible to interrogate changes in protein function in response to identified post-translational changes to provide a needed predictive understanding essential to the redesign of biological systems that are critical to the implementation of synthetic biology approaches. Using such information, targeted imaging measurements can validate the mechanistic underpinning of observed correlations to provide a means for real-time measurements of cellular reprogramming in response to environmental change. These latter measurements, coupled with a structural understanding of the protein complexes, provide a basis for synthetic biology applications, such that binding interactions between protein complexes can be adjusted to modify energy flux within individual cells and communities. To obtain this latter predictive understanding, an ability to use 'omics' information to understand and predict how cells respond to stressors using model-dependent approaches to data analysis that incorporate the current understanding of the system, and allow data integration from different sources is required. This latter goal will provide a basis to understand complex biological, climatic, and environmental systems across vast spatial and temporal scales, a fundamental BER goal, which indicates a need to understand how genomic information is translated with confidence to redesign microbes, plants or ecosystems (http://science.energy.gov/).

Combining 'omics' approaches with advanced imaging and structural biology is necessary to probe the organizational principles of biology involving environmental sensing, energy transduction, and chemical storage systems to develop the fundamental design principles that control molecular interactions to regulate living systems. Only through integrated approaches that include proteomics, structural biology, and high-resolution imaging will it be possible to understand how cells balance dynamic needs for synthesis, assembly, and turnover of cellular machinery in response to changing environmental signals. Parallel measurements of these integrated processes are necessary to obtain the predictive ability necessary for synthetic redesign that will enhance an ability to introduce multi-component biological functional modules and manipulate genetic control systems. Again, high-throughput measurements, such as those possible using UTEM (see below), are critical to these goals. They offer an ability to validate biological function and facilitate the interoperability of biological modules and processes in both lab-based and environmental systems. 


\subsubsection{Limitations of Available In Situ Biology Capabilities}

Current high-resolution structural capabilities focus on measurements of the structure and dynamics of proteins and other biomolecules following cellular disruption, thereby resulting in a loss of full biological context. Structural tools that extend an understanding of individual molecular components of cellular networks through in situ measurements of function in living cells are needed. In the case of proteins, an important opportunity is to build upon available and anticipated large increases in high-resolution structural information available from the five new national user facilities for structural biology (http://science.energy.gov/ber/research/bssd/structural-biology/), creating a meaningful context to understand metabolic control and synthetic capabilities of, for example, individual microbes within their community structures - goals that are highly relevant to predictive and synthetic biology applications. In the case of predictive biology applications, current imaging methods typically are limited to the identification of the co-localization of proteins that have been engineered to contain large fluorescent protein reporter groups (that commonly interfere with function, prevent targeting of bacterial proteins across the inner membrane, and require oxygen for their maturation). These capabilities now are possible in microbes due to advances in super-resolution imaging, which permits 10-20 nm resolution $^{40}$. Limited attention has focused on high-resolution structures of biomolecules for in situ measurements aimed at understanding living systems. In this respect, newly developed structural tools due to come on-line at Brookhaven National Laboratory use high-energy x-rays to create hydroxyl radicals that offer a means to identify changes in solvent exposure of sequences within proteins as a means to understand protein structural changes. A major advantage of these methods is that analysis does not require protein engineering to introduce a spectroscopic label. However live-cell measurements of cell function are not possible because analysis requires cell disruption, and oxidative damage to proteins can substantially modify their structure and function. Furthermore, effective implementation of these latter x-ray footprinting approaches requires quantitative mass spectrometry approaches to map levels of modification onto proteins. These limitations currently allow limited proof-of-principle applications involving the targeted analysis of individual proteins. Still, when combined with advanced modeling approaches these methods provide a means to identify binding interfaces for targeted and molecular evolution approaches used for protein redesign that offer a means for fine-tuning energy flux through pathways to favor synthetic biology applications.

New methods, such as in situ liquid TEM of proteins and UTEM, are needed that permit real-time measurements of protein function in living cells. Such measurements will permit an ability to understand community changes in metabolic capabilities in response to environmental conditions and to aid in the construction of realistic molecular models of metabolism and the identification of enzymes and/or substrates with desired catalytic activities/sensitivities. These experiments will contribute to ongoing work at PNNL that includes measurements aimed at understanding the formation of lignin along the inside of cell walls or biomineralization with applications to $\mathrm{CO}_{2}$ fixation to address coordinated programs involving other heterogeneous biological systems of DOE importance. While other general approaches have been proposed to address these challenges, in all cases there are major limitations associated with either inherent structural or dynamic resolution. For example, Argonne National Laboratory proposed a general approach to using vibrational spectroscopies that identify unique protein signatures to map location and structural dynamics of a range of proteins within living cells. However, this method currently lacks sufficient sensitivity for targeted approaches of cell functions involving all but the most abundant biomolecules (e.g., lipids or amyloid deposits involving unique beta sheet signatures). Alternative targeted in vivo approaches, as developed by Roger Tsien, involve the development of chemical probes that can be used to site-specifically label multiple proteins to allow real-time measurements of protein location and functional motions, albeit with limited structural resolution. Extending measurements to high-throughput structural determination, and correlations between protein sequence, structural dynamics, and binding affinities involving subunits of oligomeric (supramolecular) complexes identified as sites of regulatory control is uniquely possible using DTEM, which provides the needed spatial and temporal resolution to integrate measurements across scales for both individual parts of cells and community interactions between different cell types in natural communities. The proposed capability builds on current 
structural genomics measurements involving high-resolution structure determinations using both solution nuclear magnetic resonance (NMR) methods and x-ray diffraction. Such structural biology insights are critical toward understanding the assembly and functional maintenance of molecular complexes prior to their export as modules in toolkits for synthetic biology applications that seek to extend initial efforts primarily focused on low efficiency proof-ofprinciple methods introducing new pathway components into model organisms (e.g., the creation of electrically conductive E. coli using introduced metal reductases originally identified in Shewanella by PNNL researchers ${ }^{41}$ ).

\subsubsection{Natural Community Systems}

Microorganisms and plants function as members of natural communities to play fundamental roles in controlling carbon and nutrient cycles in terrestrial ecosystems and represent a source of genetic diversity that encodes a range of functions capable of contributing to pressing societal needs, including $\mathrm{CO}_{2}$ sequestration, bioenergy production, and contaminant bioremediation. Currently, our ability to harness the capacities of organisms and communities is limited by fundamental gaps in knowledge regarding the diversity of biochemical pathways and functions that exist in natural systems. For example, many new biochemical functionalities almost certainly remain to be discovered. Again, as noted, a specific and major limitation in understanding biological systems is the fact that the function of at least one third of all proteins currently is unknown.

In addition to general biochemical roles, natural microbial populations host a diversity of proteins that are fine-tuned through sequence variation. At a fundamental level, effective experimental strategies are needed that address how sitespecific amino acid variations impact the dynamic structure, associations, and function of proteins. For example, sitespecific variations in sequence may modify protein domain interactions through formation of salt-bridges, or alter posttranslational control mechanisms by varying binding affinities of regulatory proteins (e.g., kinases). Likewise, sequence variations affect the sensitivities of proteins to direct chemical modification, acting to modulate sensitivities to environmental conditions involving, for example, light intensity and the formation of singlet oxygen. Such sequencespecific sensitivities can be envisaged to allow organisms to optimally inhabit a defined niche within a community structure, resulting in enhanced community stability. Integrating these measurements with an understanding of the control mechanisms that regulate cellular targeting and maintenance of proteins and other biomolecules to maintain cell function is ultimately needed to obtain a predictive understanding from genomic information. Such knowledge may be harnessed in the future to create and optimize functions enhancement via genetic engineering. Similarly, the function of many proteins requires either transient or stable interactions with other proteins to form supramolecular complexes, whose function is further modified by changes in cellular localization; protein localization is affected by associations with other cellular components (e.g., lipid membranes). Understanding cellular regulation that controls protein complex formation requires an ability to extend current high-throughput cellular measurements able to catalog proteins and their posttranslational modifications to include data-driven computational models enabled by improved structural measurements that can use available high-resolution structural data of individual proteins (and other biomolecules) to interrogate their cellular functions within natural community structures. In using available structural data of individual proteins (or complexes) in different functional states, methods are needed that address how the cellular context in a community ecosystem affects the functions of specific proteins. Such in situ measurements of cellular processes will enable the ability to monitor emerging behaviors that vary from an understanding of which protein complexes form in response to environmental change to an appreciation of the underlying mechanisms responsible for more complex phenotypes that include functional relationships between microbes in natural communities and their response to, for example, diel cycles. These measurements will provide the necessary data to enable a modeling of the metabolic capabilities of microbial communities to provide a predictive understanding and development of synthetic biology capabilities that allow manipulation of complex biological systems. 


\subsubsection{Observations of Living Tissue}

Measurements of cellular responses to environmental exposures (e.g., low-dose radiation) typically require destructive methods that fix tissues at time-points following exposures to arrest activity, preventing longitudinal measurements of cellular responses. Such limitations curtail the ability to use existing high-resolution imaging methods to measure metabolite fate and transport, which involve using analogs of common metabolites with incorporated radioisotopic tracers, providing a sensitive means to interrogate organ function through real-time measurements of uptake and turnover kinetics. Extending these measurements to understand intracellular mechanisms and their relationship to intercellular interactions (akin to community function) requires an ability to simultaneously image changes in overall cellular properties with specific changes in subcellular structure (including changes in protein complex formation).

\subsubsection{Importance of UTEM Imaging to Biology}

UTEM has the potential to provide critical insights into the fundamental design principles that control molecular interactions to regulate living systems. Of particular importance, UTEM permits measurements of structural changes at atomic resolution (about $1 \AA$ ) with nanosecond time resolution through the acquisition of an image involving data collection from individual biomolecules (thereby avoiding radiation artifacts) using newly developed ultrasensitive detectors that permit imaging of subcellular structures (including supramolecular protein assemblies) that avoid common fixation artifacts. ${ }^{42}$

Our ultimate goal is to use biomolecular structural data to map protein complexes within living cells, providing an ability to assess functional associations and their relationships to community function. UTEM capabilities offer a straightforward means to acquire high-throughput information about isolated protein complexes and provide a means to identify molecular associations and their high-resolution structures. Such supramolecular complexes include large classes of membrane proteins that currently remain refractory to structural characterization, as they are difficult to form large three-dimensional crystals required for traditional structural methods. Alternative sample preparations involving the formation of two-dimensional crystals within a planar lipid bilayer are suitable for structure determination using UTEM. The ability to analyze small samples facilitates comparisons with orthogonal techniques commonly used to isolate and identify protein complexes, such as affinity pull-downs and mass spectrometry, affording an assessment of heterogeneity of protein associations in response to environmental exposures. Because these associations modify the interface with the environment to affect cellular signaling, such measurements are of particular interest for membrane proteins.

High-throughput approaches that screen large numbers of constructs to assess function and possible relationships to overall structural composition are expected to permit synthetic biology applications involving, for example, reconstitution of large multi-enzyme collections (such as metal reductase complexes and cellulosomes) that may aide in biofuel applications. More detailed structural measurements applicable in situ for cells are possible following cryogenic freezing. Extension of these methods to consider entire organisms is proposed using cryogenic methods to image samples tens of microns in size and thickness, which may help measure polymeric structures in living cells (such as lignocelluloses) to identify mechanisms that overcome its recalcitrance to degradation for biofuel applications. At the community structure level, UTEM provides the unique ability to quantify three-dimensional structures of biofilms with nm scale spatial resolution.

Ultimately, a major advantage of UTEM is the ability to probe conformational heterogeneity within macromolecular complexes both in solution and within living cells. Using single molecule diffraction methods, larger (and more interesting) biomolecular complexes are readily resolved due to their slower rotational motions. Fast exposure times 
(relative to rates of rotational diffusion) are possible with ultrafast femtosecond pulses that eliminate motional averaging, permitting resolution of a large range of conformational states important to imaging biological function.

Additional advantages of UTEM, and example applications that highlight how UTEM will permit measurements of biological processes across scales, ranging from those associated with enzyme reaction mechanisms $(0.1-0.3 \mathrm{~nm})$, supramolecular complex formation $(1-100 \mathrm{~nm})$, and cellular transport and targeting (0.5 microns) to community interactions (10 microns), are described (as follows).

\subsubsection{Expectations of UTEM Capabilities for Structural Measurements Across Scales}

UTEM has the potential to make time-resolved measurements of protein dynamics on the nanosecond time-scale with $1 \AA$ resolution, offering enhanced resolution to any extant approach, including those made at the SLAC National Accelerator Laboratory (SLAC) where x-ray FELs permit resolution of $8.5 \AA$ structures of supramolecular complexes (PSII reaction center) using nanocrystals and the ability to resolve 32-nm resolution of large virus particles from diffraction patterns generated with a single $\mathrm{x}$-ray pulse for the virus in solution (non-crystalline samples). Accordingly, UTEM is expected to permit measurement of high-resolution structures of individual proteins, macromolecular complexes, organoids, and viruses in solution without the need for crystallization. These new methods may enable "diffract and destroy" imaging where the diffraction pattern is collected for individual molecules prior to the onset of radiation induced damage or motion. Ultimately, it is anticipated that these new methods will enable the creation of movies of proteins undergoing catalysis, as well as the identification of macromolecular structures in viruses or intact living microbes. In comparison with other approaches that typically require large sample volumes (e.g., $10 \mathrm{~mL}$ of solution at $1 \mathrm{mg} / \mathrm{mL}$ or $10 \mathrm{mg}$ of nanocrystals), UTEM requires significantly smaller volumes of dilute solution. When operational, these reduced sample requirements will avoid the need to construct specialized infrastructures associated with large-scale cloning and expression of functional supramolecular protein complexes.

\subsubsection{Biocatalysis and Enzyme Reaction Mechanisms}

Synthetic biology approaches aimed at mediating climate change are of considerable interest, and require an understanding of natural microbial systems capable of catalyzing carbon fixation, such as formate dehydrogenase $\left(\mathrm{FDH}_{\mathrm{H}}\right)$. A molecular understanding of these natural mechanisms will guide the synthesis of effective molecular catalysts (http://iic.pnnl.gov/), which currently are unable to catalyze $\mathrm{CO}_{2}$ reduction to formate, to create mimics that like $\mathrm{FDH}_{\mathrm{H}}$ are able to catalyze carbon fixation ${ }^{43,44}$. Under biological conditions, $\mathrm{FDH}_{\mathrm{H}}$ exists as part of a formate-hydrogen lyase complex that decomposes formate to hydrogen and carbon dioxide under anaerobic conditions in the absence of exogenous electron acceptors ${ }^{45}$. This reaction is readily reversible. Therefore, $\mathrm{FDH}_{\mathrm{H}}$ promotes $\mathrm{CO}_{2}$ reduction (a difficult reaction) and permits electrochemical reduction of carbon dioxide in a manner that avoids excessive overpotentials (which are energetically wasteful) with chemical specificity not possible using non-protein catalysts, which result in a mixture of products ${ }^{43}$. As a result, understanding the reaction mechanism of $\mathrm{FDH}_{\mathrm{H}}$ is important for the design of effective catalysts for $\mathrm{CO}_{2}$ fixation. In this respect, $\mathrm{FDH}_{\mathrm{H}}$ from $E$. coli represents one of the best-understood formate dehydrogenases whose mechanism is consistent with the goal of understanding the design of biomimetics for $\mathrm{CO}_{2}$ fixation. The structure of $\mathrm{FDH}_{\mathrm{H}}$ was first solved in 1997 and subsequently corrected in $2006^{46,47}$. The presence of a selenocysteine (SeCys) ligand to the Mo metal, where the Se-Mo binding is thought to be critical to efficient catalysis, is central to the reaction mechanism. Substitution of SeCys with a Cys at this position results in a $99 \%$ reduction in catalytic rate, which may be related to the complete ionization of SeCys $\left(\mathrm{pK}_{\mathrm{a}}=5.3\right)$ and increased nucleophilicity of selenium as compared with sulfur that promotes nucleophilic attack following formate binding ${ }^{48-50}$. The active site of $\mathrm{FDH}_{\mathrm{H}}$ comprises a SeCys, Mo, two 
molybdoperterin guanidine dinucleotides (MGD), and a single [4Fe-4S] cluster, which catalyzes the two-electron oxidation of formate to $\mathrm{CO}_{2}{ }^{46}$. An inorganic sulfur $\left(\mathrm{S}_{\mathrm{i}}\right)$ ligand is bound to Mo (at least in some states), and may participate in modifying the reactivity of the active site. The reduction mechanism involves $\mathrm{SeCys}{ }^{140}$ and $\mathrm{His}^{141}$ in the rate-limiting step of proton abstraction and the Mo-molybdopterin, $\mathrm{Lys}^{44}$, and the $[4 \mathrm{Fe}-4 \mathrm{~S}]$ cluster in promoting electron transfer (where the [4Fe-4S] cluster is thought to permit a sequential ping-pong one-electron transfer mechanism) ${ }^{46}$. However, while the function of the Mo-molybdopterin cofactor is clear, the precise role of the active site Se remains uncertain $^{46,47,51}$. The high-resolution structures of $\mathrm{FDH}_{\mathrm{H}}$ in the reduced $\left(\mathrm{Mo}^{\mathrm{IV}}\right)$ and oxidized $\left(\mathrm{Mo}^{\mathrm{VI}}\right)$ states illustrate that Mo coordinates four cis-dithiolene sulfurs of the MGD cofactors and the Se of SeCys ${ }^{140}$ in a square planar geometry (reduced state), which upon oxidation assumes a trigonal prismatic coordination geometry ${ }^{46}$. The loop ${ }^{138} \mathrm{RV}$-SeCysHGPSVA $^{146}$ is weakly diffracting for $\mathrm{FDH}_{\mathrm{H}}$ following formate oxidation and the reduction of the Mo active center, and has been reported to be incorrectly assigned in the original structure (1aa6.pdb). Reinterpretation of the diffraction data has led to an altered mechanism involving a large-scale movement of this loop resulting in a $12 \AA$ separation between Se in $\mathrm{SeCys}^{140}$ and the Mo(IV) metal center (2iv2.pdb) in reduced $\mathrm{FDH}_{\mathrm{H}}$ relative to the oxidized Mo(VI) form ${ }^{47}$. What remains uncertain is: 1) the role of loop mobility and SeCys bond formation with Mo in promoting formate oxidation, proton abstraction, and electron transfer from the metal center to the $[\mathrm{Fe} / \mathrm{S}]$ cluster, 2) the precise role of SeCys ${ }^{140}$ (or alternatively, the proximal $\mathrm{His}^{141}$ ) in mediating proton transfer from bound formate, and 3) the role of $\mathrm{Arg}^{333}$ in either stabilizing the free seleno following formate binding to $\mathrm{FDH}_{\mathrm{H}}$ (option 1), acting as a proton acceptor (option 2), or in the alignment of the formate to facilitate catalysis at the metal center (option 3) ${ }^{46,47,52}$. Understanding these steps in the reaction mechanism of $\mathrm{FDH}_{\mathrm{H}}$ will be facilitated by time-dependent structural measurements of individual reaction steps that are possible using UTEM. Structural analysis of $\mathrm{FDH}_{\mathrm{H}}$ by UTEM will be possible by growing two-dimensional protein crystals and collecting diffraction patterns at different time-points. The ability to trigger the initiation of the reaction through the release of substrate (i.e., formate) using a pump laser to activate caged compounds (e.g., phydroxyphenacyl formate) is critical to these measurements. Upon stimulation with a $300 \mathrm{~nm}$ wavelength laser pulse, formate will be released on the sub-nanosecond time-scale ${ }^{53}$, allowing use of the probe beam to collect single electron diffraction patterns at various times following photoactivation. Analysis of a series of diffraction patterns from both types of experiments will provide information about protein structure and how it changes during the reaction cycle, permitting both experimental validation of the proposed structure and an understanding of how the loop structure (and SeCys) modulates catalysis (Figure 5).

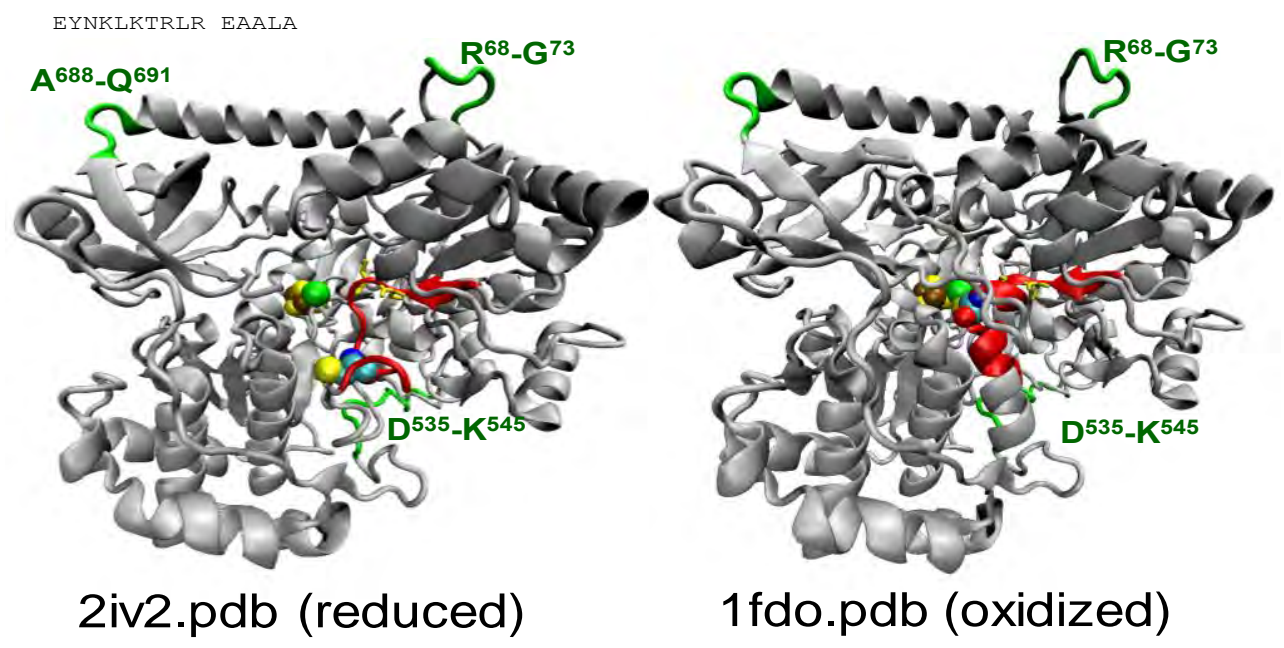

Figure 5. High-resolution structures for $\mathrm{FDH}_{\mathrm{H}}$, highlight ingSeCys ${ }^{140}$ (space-filling representation) on flexible loop structure $\mathrm{Val}^{133}-\mathrm{Ala}^{146}$ (red), positions of Mo (green sphere) and [4Fe/4S] cluster (yellow), and flexible loops designated for engineering probe labeling sites (green). 


\subsubsection{Structural Regulation Through Redox Control Mechanisms and Post- Translational Covalent Protein Modifications}

Understanding molecular mechanisms that underlie community interactions and their stability in response to environmental change (e.g., light intensity, oxygen concentrations, and nitrogen availability) requires an appreciation of regulatory controls that act to coordinate intracellular metabolic shifts to maintain requisite species diversity. Such shifts include relatively straightforward mechanisms involving well-understood transitions between aerobic and fermentative metabolisms involving transcriptional regulation through oxygen or nitric oxide binding to heme or $4 \mathrm{Fe} / 4 \mathrm{~S}$ clusters in protein sensors $^{54,55}$. Such sensors act over relatively long time-scales, and include histidine-kinases or electron transport regulator A (etrA) that act to coordinately regulate gene expression to reprogram cellular metabolism ${ }^{56,57}$. Such changes in transcriptional activation involve formation of supramolecular protein complexes that include the reprogramming of RNA polymerase binding affinities to favor transcription of different operons ${ }^{58,59}$. Identification of the signatures of key regulatory control elements available from the proposed complementary systems level measurements will enable the construction of predictive models of microbial metabolism. What remains largely unclear is the mechanism of the rapid response regulators to environmental change that allow proximal organisms in microbial communities to maintain optimal growth rates and function under highly variable and changing environmental conditions involving, for example, the high light levels (1700 microeinsteins $/ \mathrm{m}^{2} \mathrm{sec}$ ) and oxygen concentrations (i.e., 600\% air saturation) observed in Yellowstone National Park microbial mats. In this respect, there is a well understood adaptive value in maintaining long-lived proteins (such as the outer membrane metal reductase MtrC in Shewanella oneidensis) that retain fundamental metabolic capabilities under changing environmental conditions ${ }^{60-62}$. For this reason, metabolic shifts in response to environmental change are hypothesized to involve reversible post-translational modifications (PTMs) involving a limited subset of sensor proteins involving both traditional kinase/phosphatase signal transduction cascades as well as redox-dependent mechanisms involving reversible oxidative modifications. Redox-dependent mechanisms have been identified to include disulfide bond formation, methionine oxidation, and formation of nitrotyrosine - which are all known to be reversible through enzymatic control mechanisms ${ }^{63,64}$. Extension of conserved mechanisms documented in model systems under controlled cultivation to natural communities is anticipated to permit an appreciation of key cellular sensors that act to coordinate observed differences in metabolic sensitivities between different organisms to environmental conditions that allow proximal organisms in mat communities to exhibit different optimal metabolic efficiencies during the diel cycle ${ }^{65}$.

As indicated above, reversible changes in the oxidation state of redox sensors result in structural changes and alterations in protein function, representing key regulatory points in cell metabolism to enhance energy efficiencies and cell survival under rapidly changing environmental conditions (e.g., $\mathrm{O}_{2}$ content, light intensity, and nitrate levels result in metabolic shifts in phototrophs). Likewise, post-translational protein modifications involving, for example, phosphorylation commonly induce changes in protein associations that are reflective of changes in cellular metabolism. Using UTEM to monitor structural changes involving protein conformation and macromolecular associations following affinity isolation of trapped complexes from lysates will provide a rapid means to assess changes in metabolic capability, and relationships to metabolic function. Notably, we emphasize that current measurements that identify protein modifications are unable to provide a functional correlate, because samples are typically analyzed following proteolytic digestion and loss of structural information. In contrast, an ability to couple a high-throughput structural tool in concert with standard sample identification strategies offers a means to use orthogonal structural information to identify the important regulatory elements that can be used to construct predictive models. Such measurements have considerable importance to ongoing efforts aimed at understanding molecular mechanisms used by natural communities to control metabolic flux through dominant pathways that function to mediate energetic efficiencies and stability. This includes an understanding of metabolic exchange mechanisms involving: 1) secretion of glycolate upon increased rates of photorespiration by RuBisCo under conditions of high light intensity or 2) secretion of acetate and formate derived from fermentative metabolic breakdown of glycogen at night when mat becomes anoxic. These latter measurements are directly relevant to mechanistic 
measurements of the formate dehydrogenase (see above). Based on preliminary data, it is hypothesized that there are critical switch points that act to redirect redox equivalents between different pathways associated with, for example, carbon fixation and reductive biosynthesis or the formation of proton motive force and adenosine triphosphate (ATP) synthesis. Determination of mechanistic relationships between observed environmentally induced changes in the specific activities of central light harvesting proteins (e.g., PsaA and PsaB in photosystem I) and electron transfer proteins (e.g., cytochrome $c_{6}$ ) that link photosynthetic electron capture, reductant partitioning, and respiratory proteins associated with formation of the proton motive force and ATP synthesis to the release (secretion) of extracellular metabolites will offer unprecedented discoveries of molecular-level processes used by microorganisms to couple intracellular metabolic activities to observed changes in the abundance of extracellular metabolites, and their relationship to the formation of efficient and stable community organizations.

UTEM technology will provide a path forward for dynamic protein structural analysis, providing a high-throughput approach to obtain structural insights (and clues to function) for a plethora of identified proteins, which commonly exist in multiple functional states not currently experimentally accessible. Many macromolecular associations happen as a series of transient intermediates with short lifetimes. For example, a large number of sigma-factor regulators have been identified that direct RNA polymerase to form stable complexes with different promoter sequences, controlling transcription to reprogram cellular metabolism. Such a regulator has been identified in Rhodobacter involving a key regulator of RNA polymerase $\left(\right.$ sigma $^{\mathrm{E}}$ ) that selectively detects singlet oxygen to modulate transcription ${ }^{66,67}$. Similarly, there are large changes in the supramolecular composition of the RNA polymerase complex in response to environmental conditions that act to modify optimal growth efficiencies ${ }^{68,69}$. We expect that important insights can be obtained regarding cellular adaptive strategies through parallel bottom-up efforts involving the isolation of key response regulators (such as RNA polymerase complexes) with top-down global measurements of transcripts and associated pulse-chase measurements of protein turnover. By comparing such isolated protein complexes of individual microbes in isolation and following mutualistic community associations, we expect to identify key principles regarding how diverse metabolisms are coupled to enhance system stability.

In addition to conserved biochemical mechanisms for specific enzymes linked to catalysis of specific chemical transformations, genomic information from natural populations indicates a diversity of protein structures whose function and cellular regulation are fine-tuned through sequence variation. Understanding cellular regulation and community stability requires an understanding of how observed amino acid variations and specific post-translational modifications within protein families (typically measured using mass spectrometry) impact the structure, binding interactions, and catalytically important motions linked with key steps involving enzyme function (e.g., catalysis, sensing, or transport). In the future, such knowledge may be harnessed for enhancement of function via genetic engineering. The function of many proteins also requires interactions with other proteins, complexes, or cellular components (e.g., lipid membranes). UTEM will identify how protein complexes form, the slow steps in their association, and the components required for specific functions.

Extending measurements to high-throughput structural determination and correlation between protein sequence, structural dynamics, and binding affinities involving subunits of supramolecular complexes is uniquely possible using UTEM. UTEM methods will complement existing spectroscopic and/or activity-based chemical probes, ion mobility mass spectrometry, and the computational infrastructure, allowing meaningful data integration from high-throughput data collection and predictive simulations of protein structure using both classical and quantum mechanical methods to quantitatively include contributions from metal active sites $>45 \%$ of all proteins contain bound metals or complex centers). These latter measurements build on current structural genomics measurements involving high-resolution structure determinations using both solution NMR methods and x-ray diffraction. We envision rapidly acquiring information from a wide variety of difficult-to-characterize proteins. For example, hundreds of multiheme $c$-type 
cytochromes are encoded in the genomes of metal-reducing bacteria and play critical roles in subsurface geochemistry. However, the structure and kinetics of this diverse protein family are largely unknown. Unlike other neutron/x-ray technologies, UTEM offers the ability to image arrays of proteins under defined redox conditions. Time-resolved changes in native protein structure in response to voltage alterations will provide a direct readout of interfacial electron transfer kinetics or an ability to screen for differences in redox potentials among strain variant sequences, even with small amounts of available protein. The rate-limiting steps in electron transfer processes also may be identified. These structural biology insights are critical to understanding the assembly and functional maintenance of molecular complexes prior to their export as modules in toolkits for synthetic biology applications that seek to extend initial efforts that primarily focus on low-efficiency, proof-of-principle methods that introduce new pathway components into model organisms, for example, electrically conductive E. coli created using introduced metal reductases originally identified in Shewanella. ${ }^{70}$

Future opportunities that build upon available capabilities expected using UTEM instrumentation include programs to extend the understanding of individual molecular components of cellular networks through in situ measurements of function in living cells. In the case of proteins, an important opportunity is to build upon available high-resolution structural information of individual proteins (or stable complexes), creating a meaningful context to understand metabolic control and synthetic capabilities of individual microbes and their communities - goals that are highly relevant to predictive and synthetic biology applications.

In the case of predictive biology applications, current imaging methods typically are limited to the identification of the colocalization of proteins that have been engineered to contain large fluorescent protein reporter groups, which commonly interfere with function, prevent targeting of bacterial proteins across the inner membrane, and require oxygen for their maturation. Limited attention has been giving to taking advantage of high-resolution structures of biomolecules for in situ measurements aimed at understanding living systems. In this respect, newly developed structural tools, such as UTEM, offer a means to identify changes in protein dynamics and average domain architecture as a means to understand protein function and regulation within the cellular environment. A major advantage of these methods is that analysis does not require protein engineering to introduce a spectroscopic label (e.g., fluorescent protein) that can disrupt formation of active complexes.

\subsubsection{UTEM Permits Measurements of Assembly and Functional Dynamics for Molecular Machines}

High-impact applications of UTEM will provide important insights regarding the efficient energy transduction mechanisms related to different classes of energy transformation, including the assembly and molecular actions of supramolecular protein machines and interfacial electron transfer mechanisms to mineral surfaces. Such activities are broadly relevant to a predictive understanding of microbial communities and synthetic biology applications. Timeresolved UTEM measurements that resolve catalytically important protein motions linked to function are essential to molecular understanding. Such measurements provide the necessary linkage between atomic-level protein movements and steps in the catalytic mechanism. Likewise, experiments that target an understanding of the mechanisms responsible for the assembly and maintenance of extracellular supramolecular complexes, such as MtrABC metal reductase systems that mediate electron transfer to extracellular minerals, are importance for a range of crucial processes that include bioremediation and synthetic biology applications as the molecular machinery is likely to be highly conserved and applicable to exporting various molecular cassettes (modules) to create desired functionalities that both test community interactions and are relevant for bioenergy applications.

The metal reductase system originally identified in Shewanella, now found to be more widespread, has the potential to reveal fundamental regulatory processes important to both biogeochemical processes and microbial community 
interactions, which commonly require trafficking specific proteins to the outer membrane that mediate a host of activities associated with metabolism (e.g., terminal electron acceptors), defense, and possible mutualistic associations underlying environmental stability. In addition, the metal reductase system and other enzyme systems, such as the hydrogenase system, act to mediate bioremediation through the biogeochemical reduction of actinides during bioremediation, such as the reduction of $\mathrm{U}(\mathrm{VI})$ and $\mathrm{Tc}(\mathrm{VII})$ into less soluble oxide forms (e.g., $\mathrm{U}(\mathrm{IV}) \mathrm{O}_{2}$ and $\mathrm{Tc}(\mathrm{IV}) \mathrm{O}_{2}$ ). Newly released structures are available for the terminal subunit of the metal reductase, $\mathrm{MtrF},{ }^{71}$, and time-resolved spectroscopic measurements indicate an important role for subunit dynamics in promoting electron transfer following association with the metal oxide surface $^{72}$. Building on these insights, UTEM measurements will interrogate the molecular steps involved in interconversion of aqueous $U$ species to initial precipitates by $c$-type cytochromes, and how these steps vary among different cytochrome variants. Typically, first formed biogenic mineral products are nanoparticles $\sim 1.5-2.5 \mathrm{~nm}$ in diameter $^{73}$, although the formation of molecules and clusters also has been proposed. Using UTEM, we anticipate ps- $\mu \mathrm{s}$ measurements of the rate, aspects of the mechanism, and products of reduction reactions using isolated protein complexes in aqueous solution. Experiments take advantage of the diversity of cytochrome variants recovered both from single organisms and strain variants. Specifically, we foresee measurements for proteins in the presence of aqueous actinide ions in a fluid cell after photolysis of caged reagents allows reduction to proceed. Coupled with quantum mechanics/molecular mechanics, or $\mathrm{QM} / \mathrm{MM}$ ) calculations, we anticipate having the ability to identify the molecular switches that control electron transfer rates as electron transfer efficiencies are dramatically enhanced upon small changes in cytochrome orientation.

An understanding of the hydrogenase enzyme system, which functions to catalytically mediate proton-coupled electron transfer mechanisms to form hydrogen and has been shown to reduce $\mathrm{Tc}(\mathrm{VII})^{74}$, also is relevant to bioremediation. The availability of high-resolution crystal structures of hydrogenase enzymes stabilized in specific intermediate states will enable analysis of how function is conferred by conformational changes. For example, time-resolved measurements of dynamic structural intermediates with high spatial resolution (1-3 $\AA$ ) could track reactions initiated by rapid changes in electrochemical potentials or photoactivation, following engineering of enzymes to contain site-specifically bound photosensitizers. These measurements provide a means to interrogate how changes in the geometry around active site metals are coupled to the protein matrix, permitting direct measurements of catalytic mechanisms that control the activity of the metal center (e.g., the hydricity of the active site in hydrogenase enzymes, which are predicted to arise through first and second coordination sphere influences of the protein that alter bite angles). Furthermore, changes in protonation states of sites proximal to hemes or $\mathrm{Fe} / \mathrm{S}$ clusters are expected to influence their relative geometries and electron transfer efficiencies, providing a means to understand how electron flux is regulated. Such measurements will reveal mechanistic insights critical for efficient hydrogen production at low potentials with minimal side reactions commonly associated with catalytic inactivation and will be critical to the eventual synthesis of inorganic catalysts.

\subsubsection{Protein Synthesis}

The UTEM will enable a variety of time-resolved studies of protein complex formation and behavior. For example, ribosomes, sites of protein synthesis in the cell, are highly dynamic 2.5 MDa ribonucleoprotein machines. Protein synthesis on the ribosome can be separated into four major steps - initiation, elongation, termination, and recycling - all of which have been the subject of intense study. Structurally, the current state of the art for cryo-electron microscopy allows for time resolution on the order of $5 \mathrm{~ms}$, which is limited by the rate of plunging the specimen into cryogen. Reaction steps such as release of initiation factor 2 during initiation; elongation factor-G (EF-G)-dependent translocation; and ribosomal recycling, where guanosine triphosphate (GTP) is hydrolyzed, can be triggered in the UTEM using flash photolysis of caged GTP with a latency before image acquisition as short as 1 ns. Alternatively, use of a stopped-flow-like mixing cell would have a longer latency, on the order of $\mu \mathrm{s}$, but would allow for visualization of a more general class of chemical reactions (i.e., not limited to those involving photolabile reactants). In either the flash photolysis or stopped- 
flow-like configurations, a time series can be collected in movie mode with the time between exposures determined by the kinetics of the reaction under investigation.

\subsubsection{Cultivating Microorganisms}

Many microorganisms have defied cultivation attempts. Community metagenomic measurements have provided insights into organismal diversity within natural systems, permitting hypotheses to be developed regarding their underlying metabolic potentials. However, topological information, including details of the ultrastructure of uncultivated cells and their interactions with other community members, is lacking from these studies. When natural microbial communities have been surveyed by TEM, distinct structures and organelles are often recognized ${ }^{75,76}$. Information about membership, cellular features, and community organization information is vital because nutrient, biochemical, and other transformations almost certainly depend on consortium-level phenomena that are not well captured through studies of isolated cells.

Single-particle cryo-TEM and cryo-electron tomography have increasingly provided three-dimensional structural information, bridging the information gap between knowledge of the existence of a genomically encoded protein and structure-function relationships. Cryo-TEM will be an important platform for UTEM. The short-pulse, high-flux capacity of dynamic TEM will significantly enhance the ability to monitor structural dynamics and relationships to function by providing significantly higher signal-to-noise images and higher information accuracy. Specifically, we envision applying this approach to better characterize enigmatic organelles, such as tubular structures, localized macromolecular complexes, viral infection stages and features, polysaccharide, and other cell-associated polymers. The approach can be applied to as yet unidentified cells and be used to characterize organism-organism interaction structures. ${ }^{77}$

\subsection{Applications of Ultrafast Electron Microscopy in Biology and Biogeochemistry}

Many important biological and environmental phenomena depend on transformations in electronic and/or atomic configurations. While materials (e.g., particles) or biological organisms (e.g., microbes) may remain static under native environmental conditions, they still may play critical roles in a variety of environmental and biological processes involving, for example, biogeochemical cycles and atmospheric transformations associated with air pollution (smog). Understanding such processes is pertinent to a range of practical problems associated with comprehending atmospheric and occupational environments, which includes fundamental concerns involving catalysis, technologies related to energy production and emissions control, particle nanotoxicology, and health effects. A fundamental understanding of these processes requires advanced analytical approaches for chemical imaging of particles down to sub-nanomolar spatial resolutions in reactive environments. The key transients in all of these processes can take place on a time-scale that extends from microseconds to femtoseconds and on a length scale that can be below a nanometer (in some cases extending to the atomic scale). UTEM capabilities provide an ideal platform to probe these phenomena through a combination of imaging, spectroscopy, and diffraction on their natural length and time scales. In this section, the science needs are discussed that require time-resolved measurements in areas including that include: 1) the biotransformation of multivalent metals and radionuclides; 2) naturally forming clusters, nanoparticles, and colloids, which are especially important in biological and environmental science; 3) engineered nanostructures that influence many different areas, including atmospheric aerosol science, biological materials particles interaction, and energy materials; and 4) investigating proteins in supramolecular assemblies and cells. 


\subsubsection{Biotransformation of Actinides or other Multivalent Metals}

Biogeochemical processes related to changes in mineral formation are coupled to microbial processes, as well as the reduction of toxic metals to diminish their solubility to reduce contaminant movement in the subsurface environment. An important question in biogeochemistry relates to the mechanism and rate of biotransformation of actinides during bioremediation (e.g., reduction of U (VI), Tc (VII), and $\mathrm{Cr}(\mathrm{VI})$ ) into less soluble oxide forms $-\mathrm{U}(\mathrm{IV}) \mathrm{O}_{2}, \mathrm{Tc}(\mathrm{IV}) \mathrm{O}_{2}$, and $\mathrm{Cr}$ (III) nanoparticles. Relatively little is known about the molecular steps involved in interconversion of aqueous uranium species to form initial precipitates by $c$-type cytochromes, such as MtrC and OmcA. Even less is known about how these vary among other U(VI)-reducing cytochromes. Typically, first-formed biogenic mineral products are nanoparticles $\sim 1.5$ $\mathrm{nm}$ to $2.5 \mathrm{~nm}$ in diameter, ${ }^{75,78-81}$ although the formation of molecules and clusters also has been proposed. In the UTEM, we anticipate ps- $\mu$ s measurements of the rate, aspects of the mechanism, and products of reduction reactions using isolated protein complexes in aqueous solution. The experiments take advantage of the diversity of cytochrome variants recovered both from single organisms and strain variants. Specifically, we foresee measurements for proteins in the presence of aqueous actinide ions in a fluid cell after photolysis of caged reagents allows reduction to proceed.

There has been major progress in rapidly acquiring information from a variety of difficult-to-characterize proteins. For example, hundreds of multiheme $c$-type cytochromes are encoded in the genomes of metal-reducing bacteria and play critical roles in subsurface geochemistry. These cytochromes often work together and mediate electron transfer to form a pathway that mediates the reduction of metals, including actinides. One of the best-characterized electron transfer pathways formed by cytochromes is the Mtr pathway of Shewanella oneidensis MR-1, which includes four $c$-type cytochromes - CymA, MtrA, MtrC and OmcA - and a trans outer-membrane and porin-like protein MtrB. Electrons move from the quinone/quinol pool in the inner-membrane, through the periplasm and across the outer-membrane to the surface of Fe(III) oxide-containing minerals as well as actinides in solution (Figure 6). Despite their important roles in biogeochemical transformation of metals and radionuclides, the structure and kinetics of this diverse protein family are largely unknown. Furthermore, their association within protein complex is even less understood. Unlike other electron/xray technologies, UTEM offers the ability to image arrays of proteins under defined redox conditions using potentiostatically controlled graphene sheets in fluid-filled stages.

As noted, time-resolved changes in native protein structure in response to voltage alterations can provide a direct readout of interfacial electron transfer kinetics as well as an ability to screen for differences in redox potentials among strain variant sequences, even with small amounts of available protein. Quantitative measurements of structure functional relationships can identify the rate-limiting steps in electron transfer processes. UTEM also provides a potential "game changing" way to determine the structures of these proteins and proteins complexes. Structural determination of these protein complexes, and relationships between protein movements to catalytic rates will enable an unprecedented molecular-level understanding of regulatory control mechanisms important to optimal function.

Current high-resolution microscopy techniques require fixation (cryogenic, chemical or resin based), which arrests activity and induces ultrastructural artifacts that prevent accurate measurements of biologically relevant structures. UTEM is unique because it allows the study of biological macromolecules in fluid-filled stages with collection of data before and after stimulation by various forms of energy, using electron pulses on time-scales faster than Brownian motion. Thus, UTEM will enable data acquisition from molecules and cells under physiological conditions with the high spatial resolution and time-resolution to enable the measurement of fundamental mechanisms underlying a range of biological biological processes on relevant time-scales. During the ultrafast imaging, microbial cells in the in situ liquid stage will be exposed to a limited electron dose $\left(<10 \mathrm{e} / \AA^{2}\right)$ to prevent significant radiolysis damage from occurring due to electron irradiation and effecting cellular function. To improve the probability of capturing the process, a small subset of consecutive frames will be collected after the first laser-pulse trigger prior to the onset of cellular damage. For example, we propose studying living cells as they interact with $\mathrm{U}(\mathrm{VI})$ and form $\mathrm{UO}_{2}$ nanoparticles by placing living bacteria in the 
in situ cell with an oxidized electron acceptor, i.e., U(VI). The caged reducing agent, (e.g., thioacetamide) will be cleaved using flash photoalysis. Upon cleavage of the reducing agent (on the ns- $\mu$ s time-scale), reduced cytochromes, such as $\mathrm{MtrC}$ and OmcA, will transfer electrons to U(VI), and temporal measurements (on the ps- $\mu$ s time-scale) of nanoparticle formation. Likewise, these experiments could be extended to include other relevant metals e.g. $\mathrm{Cr}(\mathrm{VI})$ and $\mathrm{Tc}(\mathrm{VII})$. Direct observations of nanoparticle growth in association with the cell surface will extend our understanding of the biotransformation of radionuclides.
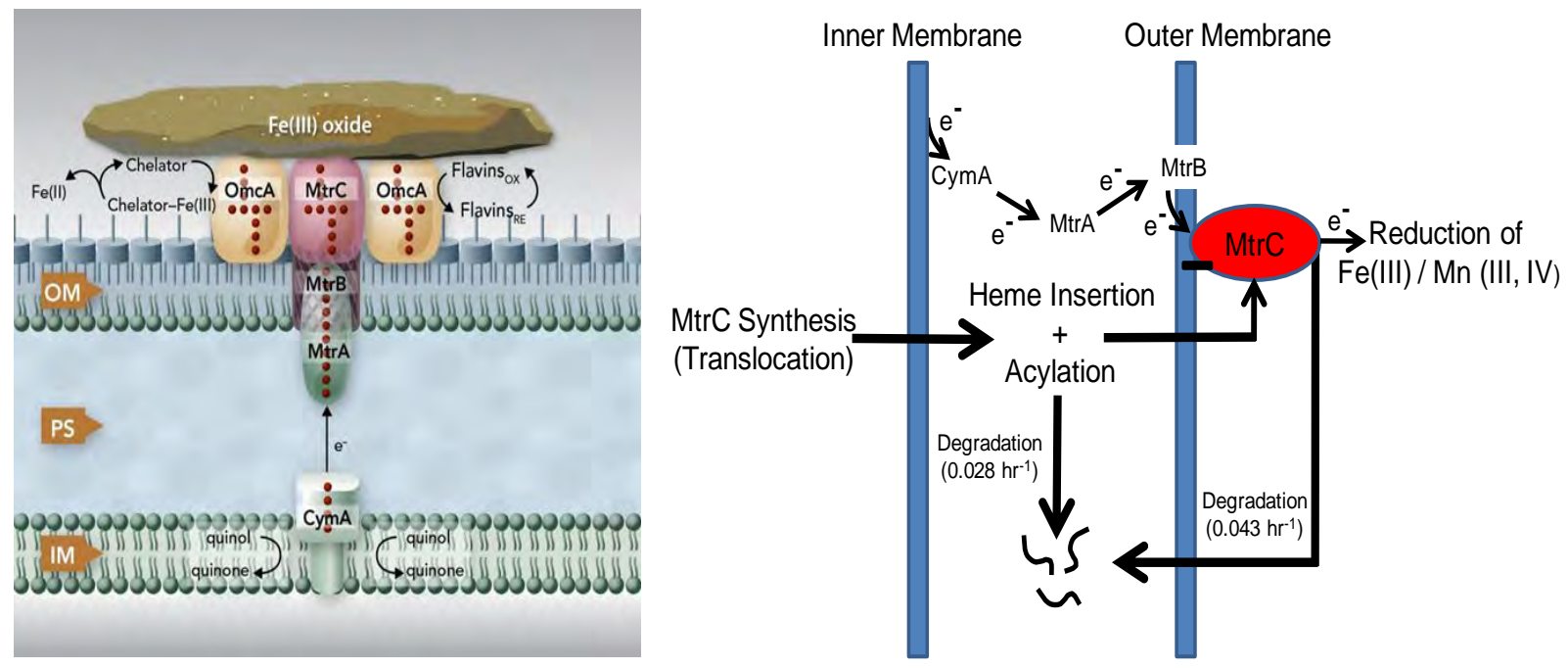

Figure 6. Cartoon illustrating membrane proteins linked to pathway involving extracellular electron transfer to the environment (left) and schematic highlighting trafficking pathway of outer membrane decaheme cytochrome MtrC (right) (Shi et al., 2007; Xiong et al., 2011).

\subsubsection{Formation of Clusters, Nanoparticles, and Colloids}

Particles of small size and high surface area dominate the chemical reactivity of environmental systems, including soils, aquifer sediments hosting groundwater, and bottom or suspended sediment in surface waters. ${ }^{81-83}$ Particles typically form in situ as a result of natural weathering and biogeochemical processes or through dissolution and/or precipitation reactions caused by anthropogenic chemical changes. Metal-oxide, sulfide, carbonate, and silicate particles can all form in association with organic matter, often exhibiting specificity in shape, orientation, and composition. Through nutrient or ion retention at interfaces, or as a result of $\mathrm{pH}$-dependent or redox control mechanisms, particulate materials affect a range of biogeochemical cycles that sustain life. Particle sizes range from the nanometer (nanoparticles) to the micron size (colloids) with hierarchical organizations often evident. These small particles are easily dispersed and transported in surface or groundwater, disseminating contaminants associated with their structure or adsorbed on their surfaces, including both radioactive materials and hydrophobic carcinogens. ${ }^{84}$ An important need facing the biogeochemical sciences is to understand how these particles form from their most fundamental chemical and biologic starting points, and the steps and mechanisms involved in their evolution to larger sizes with different structures, surface properties, and reactivity. An understanding of these processes can inform the development of new synthesis methods for engineered particles of unique function based on the mimicry of complex natural reactions and improved environmental transport models. 
While many aspects of the process of nanoparticle or colloid formation in natural biogeochemical environments is understood, significant debate exists over the dominant mechanisms of crystallization mechanisms under environmental conditions. Initially, dissolved ions and/or molecules cluster to form critical sub-nanometer-sized growth nuclei. These nuclei continue to grow via ion-by-ion collection and molecular aggregation to yield primary nanoparticles. In the classical crystallization pathway, ${ }^{85}$ continued ion collection on energetically favorable step and kink sites on growing crystal faces ${ }^{86}$ and unit cell replication leads eventually to single-crystal formation. In non-classical crystallization, the primary nanoparticles may be either structured or amorphous, and the subsequent growth pathway is particle-mediated. ${ }^{87}$ Continued crystal growth occurs by self-assembly of nanoparticles into larger superstructures (mesocrystals) by oriented attachment or aggregation. ${ }^{42,79}$ The mesocrystals can undergo further morphogenesis to yield iso-oriented or single crystals $^{88}$ —often with internal porosity, defects, or directionally dispersed amorphous materials. ${ }^{89}$ Significant debate exists over which of these crystallization mechanisms predominates in a given environment and why. Growing evidence indicates that non-classical crystal growth mechanisms are important in many natural settings, ${ }^{90}$, especially when particle formation is biologically mediated. Indeed, the non-classical growth mechanism allows incorporation of organic additives, such as biopolymers, to inorganic crystals that impart dramatic changes in physicochemical properties. These concepts underlie a new and important area of materials research: biomimetics or biologically inspired materials.

Mechanisms underlying nanoparticle and colloid formation are not well understood, in part, because of experimental limitations associated with defining the process dynamics at the sub-nanometer and nanometer scales over relevant time periods in hydrated systems. Instrumentation with sufficient temporal and spatial resolution and structural and chemical sensitivity is limited. TEM and scanning electron microscopy (SEM) have been used extensively to define nanoparticle structures, morphologies, and associations after fixed reaction periods and water removal. ${ }^{91}$ Scanning transmission $\mathrm{x}$-ray microscopy (STXM) has been applied to understand chemical and electronic distributions of environmental nanoparticles of biogeochemical origin at the nanometer scale, including the role of organic materials under uniquely in situ conditions. ${ }^{92}$ Recently, in situ liquid TEM and STEM have also been developed to directly observe nucleation and growth of nanoparticles from solution. However, the achievable time scales are limited to the millisecond range ${ }^{24,93}$. As nanoparticle nucleation and growth is strongly affected by solvation and dynamic chemical interactions at the solid-liquid interface, ${ }^{94}$ water is critical for these studies.

The important science needs to understand are:

- The formation of fundamental growth nuclei and primary nanoparticles

- The mechanisms and dynamics of particle aggregation and oriented attachment

- Protein and other macromolecular reactions at the microorganism surface yielding inorganic nanoparticles of highly defined size and crystallinity

- Morphogenic maturation processes of mesocrystals.

UTEM can play an important role in answering these fundamental questions, especially in terms of quantifying process kinetics with sub-nanometer spatial resolution of intermediate product states. Critical to a molecular understanding is the appropriate choice of conditions that permit initiation of particle formation, given the need for hydrated systems, the wide range in chemistries important in particle-forming ions, the varied biogeochemical pathways involved (e.g., redox, $\mathrm{pH}$, microorganism microenvironment effects, etc.), and the need to consider active microbiologic systems for an important subset of problems. A stop-flow cell approach or flash photolysis will have a broad range of applications for reaction initiation as these experiments typically begin by mixing reagents (e.g. ${ }^{33,95}$ to induce: 1) supersaturation or alteration in the thermodynamic stability field of a system component; 2) aggregation; 3 ) $\mathrm{pH}$, redox (e.g., $\mathrm{O}_{2}$ ), or alkalinity change; or 4) microbiologic activity and associated electron transfer (solute flux across) or materials capture by molecules attached to 
the outer cell membrane. Important mineralogical systems to consider for both abiotic and biotic experiments would include macro (e.g., Fe, Al, Mn, Ti, and Ca) and minor/contaminant element oxides, carbonates, phosphates, sulfides, and silicates. The time-resolved measurement of particle size, structure, surface morphology, and composition (if possible) to elucidate the fundamental kinetic pathways is critical to these experiments.

\subsubsection{Structure, Reactivity, and Distribution of Environmental Nanoparticles: From Aerosols to the Smallest Colloids to Confined Fluids}

\subsubsection{Environmental and Engineered Nanoparticles}

Gas-particle heterogeneous reactions play a critical role in a variety of environmental and engineering chemical processes. Such processes are relevant to a range of atmospheric and occupational environments, catalysis, technologies related to energy production and emissions control, particle nanotoxicology, and health effects. Fundamental understanding of these reaction processes requires advanced analytical approaches for chemical imaging of particles down to $1 \mathrm{~nm}$ in reactive environments. The unique analytical and imaging approach provided by UTEM will permit insights into the evolution of particle internal structures and interfaces. Use of the UTEM system will elucidate surface reactions via spectromicroscopy techniques with implicit time resolution. These measurements will offer an analytical platform to study multiphase particle reaction and in situ chemistry with broad applications in environmental and chemical engineering research. The realization of UTEM is a revolutionary step forward for dynamic chemical studies of reactant particles and will not only extend studies of particles well beyond the current capabilities but will enhance and guide complementary molecular-level spectro-microscopy investigations. ${ }^{70}$

Chemical analysis of reacting particles is an especially challenging task because no single analytical chemistry method can provide information that ranges from high-resolution microscopy to molecular characterization of complex constituents of particulate matter. The ultimate goal is to use UTEM as a novel particle analysis technique for chemical imaging to permit analysis of reacting particles. These measurements will add a vital component of time resolution that can interrogate interfacial chemical transformations obviating sample damage on the time scales of reactions down to 10 ps. This will allow researchers to bring particle analysis to an unprecedented level of sophistication and develop unique capabilities for research in the area of complex particle interfacial chemistry and in situ reactivity.

\subsubsection{Nanospace-confined Fluids}

A variety of studies have suggested or shown that the liquid confined in nanospace volumes exhibits properties different from the bulk, such as increased viscosity ${ }^{78,94,96-98}$. In addition, the diffusion of solution into the nanospaces between two adjacent nanocrystals may be retarded or inhibited. As a result, faces exposed to the confined space may dissolve more slowly than ones exposed to bulk solution. This dissolution inhibition caused by the nanospace between nanocrystals suggests that aggregation affects overall dissolution rate. When the interparticle spacing decreases further, approaching zero, oriented attachment may occur between adjacent nanocrystals. To reduce the total energy by eliminating the solidliquid interface ${ }^{99}$, chemical bonds are formed at the interface between attached particles. All of these phenomena that occur in confined spaces have been studied indirectly. UTEM, in combination with in situ liquid environmental chambers and thin fluid path lengths ${ }^{24}$, will permit study of these critical and common phenomena in real time. 


\subsubsection{Transport of Nanoparticles}

The transport of nanoparticles in the environment and other complex systems depends on size, shape, composition, and surface structure. Developing a solid conceptual framework for understanding the fate, transport, and potential toxicity of nanoparticles is essential for the assessment of impacts to the environment. ${ }^{100}$ Furthermore, understanding many of the same issues with respect to nanoparticle interactions within biological systems is even more imperative.

Nanoparticle aggregation and deposition determines the transport potential of nanoparticles, thus controlling the environmental fate and health-related impacts of these materials. There are two primary aspects that remain challenging: 1) how does the chemistry of the nanoparticle environment affect deposition and aggregation and 2) can colloidal approaches and models be scaled to nanoparticles to account for nanoscale particle interactions that, due to their small size, are inherently different than particle interactions at the micron scale? ${ }^{101}$ If these nanoparticle aggregation and deposition interactions can be understood, there is the possibility that existing models of particle transport will be successfully extended to the nanoscale with appropriate modifications. Evidence suggests the aggregation behavior of engineered nanoparticles roughly follows the classical behavior of colloidal particles in aquatic systems. ${ }^{74}$

Therefore, the key challenge is to determine the deposition behavior of nanoparticles to understand transport at reduced length scales. The need to characterize and understand nanoparticle deposition behavior in complex matrices and environments ${ }^{102}$ echoes the dilemma faced in characterizing nanoparticles. It is essential to elucidate the fundamental chemical interactions related to nanoparticle deposition approaching the true nanoscale and to satisfy the additional need for dynamic information. Advanced UTEM with specially designed, microelectromechanical system-manufactured, in situ reaction cells that can interrogate nanoparticle reaction and transport phenomena with thermal and electrochemical regulation offers a unique means to probe nanoparticle deposition and transport with the possibilities of dynamics to develop some of the first understandings of the controlling processes. This experimental information then can be used to validate theoretical transport models and predictions for reduced dimensionalities.

\subsubsection{Proposed Applications of UTEM at the Interface of Biology and Nanotechnology}

The proposed project focuses on development of complementary experimental setups that allow chemical imaging of changes in particle morphology and composition in reactive environments.

The task will include development of several types of micro-reactor cells, where the heterogeneous chemistry of particles on microscopy substrates are interrogated in situ, and under controlled conditions of gas-phase reactant(s), temperature, and relative humidity. Several approaches will be applied (but are not limited) to advance research in the following areas:

1. Atmospheric chemistry and environmental effects of airborne particles-where particle internal structure and composition have important implications for their atmospheric aging, hygroscopic, and optical properties ${ }^{70}$

2. Soot formation and soot oxidation chemistry - where interest in internal structures of soot and their chemical transformations are driven by both environmental research and technology efforts to reduce soot emissions ${ }^{82}$

3. Heterogeneous interfacial chemistry of engineered nanoparticles with a focus on the use of metal-oxide oxyhydroxide (e.g., iron/iron oxide) nanoparticles - where heterogeneous oxidation chemistry dramatically impacts their use in environmental and sensing applications, and other particles, such as nanodiamond, which are extensively used in emergent developmental biology, where functionalized nanodiamond particles can be used for cancer treatment and other health-related problems ${ }^{103-105}$ 
4. Nanotoxicology of nanoparticles - a fundamental understanding of relationships between distinct properties of nanoparticles and their cellular interactions, fate, and response in alveolar epithelial cells. Establishing predictive evaluation of inhaled non-material's toxicity or biocompatibility

5. Chemical reaction of nanoparticles in confined spaces.

As shown in Figure $7^{106}$, galena $(\mathrm{PbS})$ nanocrystals can be very close to each other within an aggregate. The interparticle spacing between adjacent $\mathrm{PbS}$ nanocrystals is as small as $1-2 \mathrm{~nm}$. This kind of nanoscale interparticle spacing is easily formed in nanocrystal clusters because of the inherent size of these particles and their crystal shapes. The confined space may affect the dissolution of nanocrystals. For example, Figure $7 \mathrm{~b}$ shows a post-dissolution nanocrystal (P1), which is adjacent to two particles (P2 and P3). The spacing between P1 and P3 is approximately 2-3 nm, while it is approximately $1 \mathrm{~nm}$ or less between P1 and P2. The partly dissolved nanocrystal, P1, presents an asymmetric shape. The $<110>$ faces on the crystal's left side in the image (denoted by white lines) are larger than the $<110>$ faces on the right side. The left-side faces are open to the bulk solution, but the faces on the right side are close to other particles. The slower dissolution rate on faces closely adjacent to other particles may be due to the abnormal properties of aqueous solution in the confined space (as discussed).
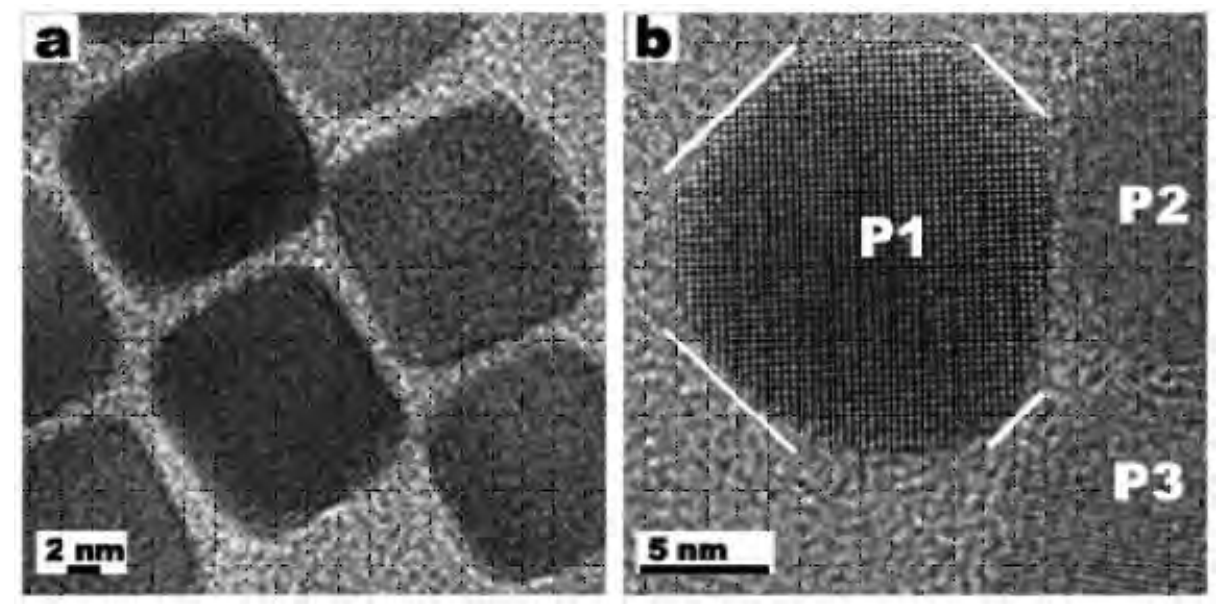

Figure 7. Nanoscale interparticle spacing formed in $\mathrm{PbS}$ nanoparticle clusters that show dissolution is slow in the confined space; (a) before and (b) after dissolution ${ }^{106}$. 


\subsection{Catalysis}

\subsubsection{Introduction: TEM and Catalysis}

The most important industrial chemical processes are facilitated by catalysts, leading to products valued in the trillions of dollars annually just in the United States. Most catalysts used in large-scale processes are solids. The 2007 DOE Basic Energy Sciences report "Basic Research Needs: Catalysis for Energy" ${ }^{107}$ makes the case for future research in catalysis, emphasizing that the science is evolving rapidly with many of the advances emerging from new and improved experimental methods for characterization of catalysts and catalysis. Much of the motivation for research in catalysis is related to energy needs. TEM is mentioned prominently among the characterization tools that will be essential for rapid advancement within this crucial field.

Solid-catalyzed reactions take place on active sites that typically comprise several atoms each. These may be nanostructures dispersed on high-area porous supports, or they may be parts of the surfaces of solids, such as corners, edges, and facets, that might be parts of nanoparticles dispersed on supports. Essential goals in catalysis science are identification and understanding of the geometric and electronic structures of catalytically active sites and elucidation of the elementary steps that constitute the reactions taking place on catalytic sites.

HRTEM has been used to image catalytic structures and catalytic sites, even with atomic resolution, and using HRTEM for catalyst characterization has increased dramatically in the last few years. Atomic-resolution images offer evidence of a marked advancement in our ability to characterize catalysts at the most relevant length scales.

\subsubsection{Images of Catalytic Species on Surfaces under Static Conditions}

Examples of atomic-resolution images of catalytic species on supports have become almost commonplace in the preceding couple of years. An example is provided by $\mathrm{BaO}$ structures on the surface of an $\mathrm{Al}_{2} \mathrm{O}_{3}$ support (Figure 8). $\mathrm{BaO}$ is an essential component of the so-called $\mathrm{NO}_{x}$ storage/reduction (NSR) catalyst, a technology recently commercialized for controlling emissions of harmful $\mathrm{NO}$ and $\mathrm{NO}_{2}\left(\mathrm{NO}_{x}\right)$ from diesel-powered vehicle engines.

Clusters of three Os atoms dispersed on an $\mathrm{MgO}$ support (Os3) are shown in Figure 9. They were made from a molecular precursor $\left(\mathrm{Os}_{3}(\mathrm{CO})_{12}\right)$ to be highly uniform and facilitate fundamental understanding of structure-catalytic property relationships. The Os-Os distances and average cluster diameters determined by STEM match those determined by extended x-ray absorption fine structure (XAFS) spectroscopy (Figure 9).

The work summarized in Figure 8 and Figure 9 demonstrates the importance of HRTEM images as inputs for theoretical calculations used to develop theoretical models of catalysts. 


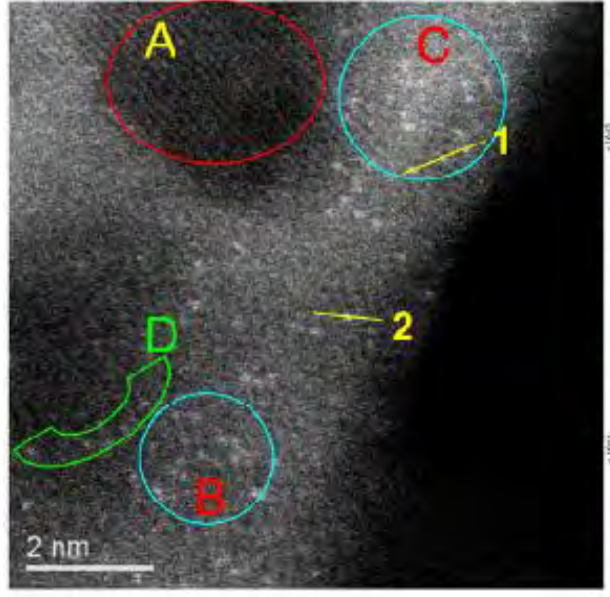

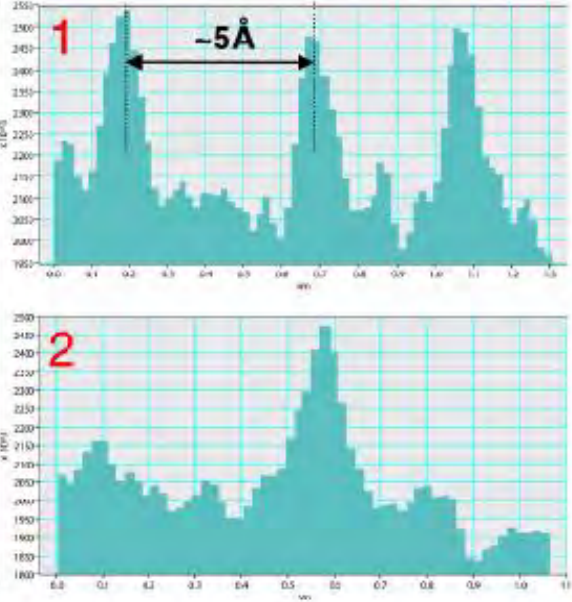

\#A

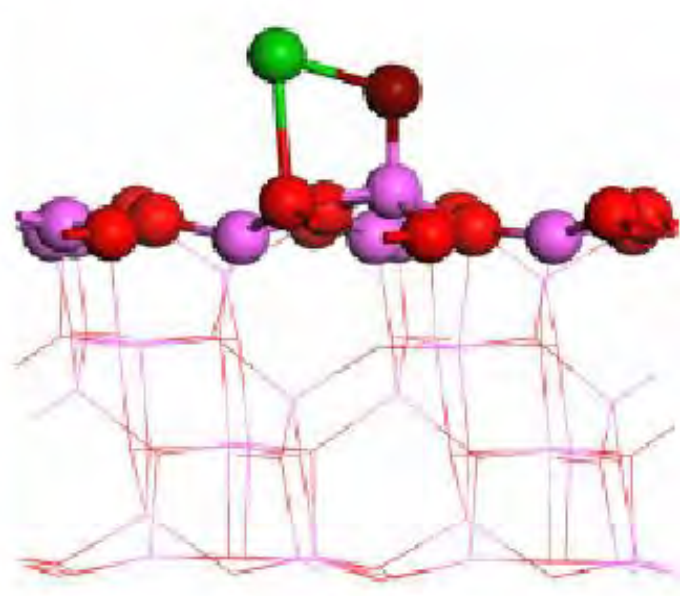

\#B

Figure 8. (\#A) High-resolution STEM image shows active monomeric catalyst structures $(2 \% \mathrm{BaO}$ on $\gamma$ $\mathrm{Al}_{2} \mathrm{O}_{3}$ ). A: No $\mathrm{BaO}$; $\mathrm{B}$, C: fairly uniform dispersion of $\mathrm{BaO}$; Line scans 1, 2: confirms single $\mathrm{BaO}$ monomers; D: $\mathrm{BaO}$ monomer decorate the facet boundary. (\#B) Computed structure of a $\mathrm{BaO}$ monomer on a dehydroxylated $\gamma-\mathrm{Al}_{2} \mathrm{O}_{3}(100)$ surface (green $-\mathrm{Ba}$; dark red - $\mathrm{O}$ in $\mathrm{BaO}$; magenta $\mathrm{Al}_{3+}$ penta and red - surface $\mathrm{O}$ atom on the $\gamma-\mathrm{Al}_{2} \mathrm{O}_{3}(100)$ surface. Bulk alumina indicated by light lines. ${ }^{108}$

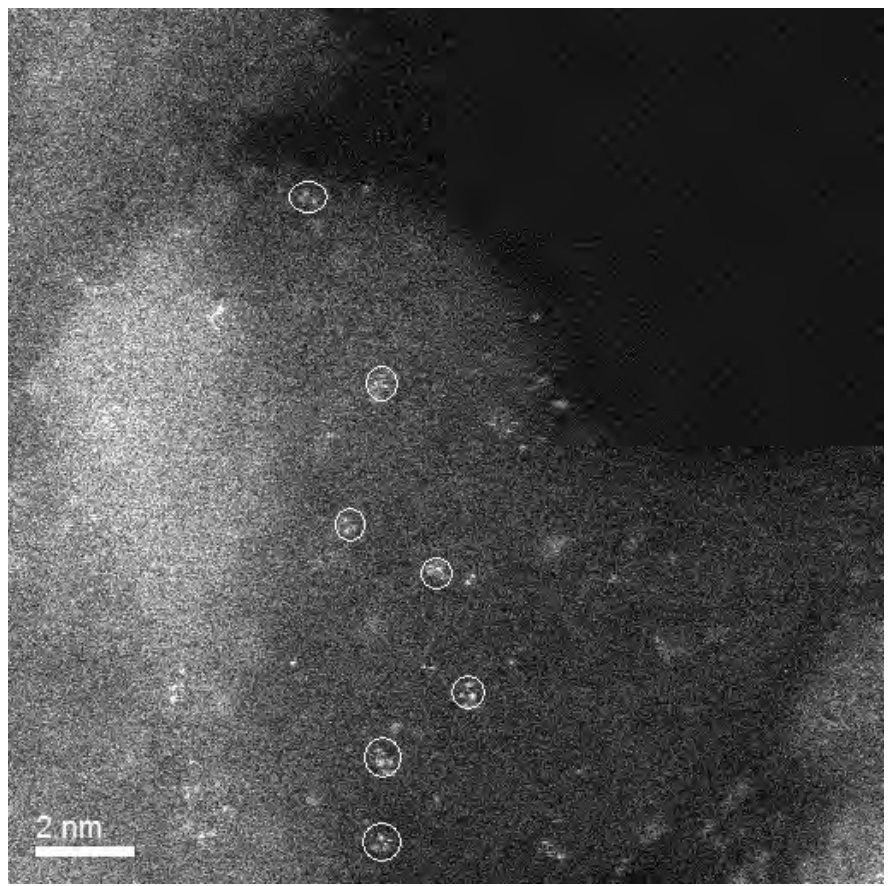

Figure 9. Image of triosmium clusters on the surface of $\mathrm{MgO}$ powder. These clusters were prepared from $\mathrm{Os}_{3}(\mathrm{CO})_{12}$. The aberration-corrected STEM images show an average Os-Os distance in the clusters of $2.80 \pm 0.14 \AA$. The distance determined by extended $\mathrm{x}$-ray absorption spectroscopy is $2.89 \pm 0.06$ Å. ${ }^{109}$ 
Because a catalytic cluster or nanoparticle is typically dispersed on a support, the interface between the catalytically active material and the support may play a critical role in catalysis. Such interfacial sites may involve only a minority of atoms in the catalytic structure. However, when clusters contain only a few atoms, each of them may be in contact with the support. Thus, it is important to discern the atoms in various positions, including the metal-support interface. STEM has been used to determine the structure of the interface in a catalyst consisting of Os3 clusters on the $\mathrm{MgO}(110)$ surface. The image is shown in Figure 10, and the corresponding structural model including the interface is shown in Figure 11.

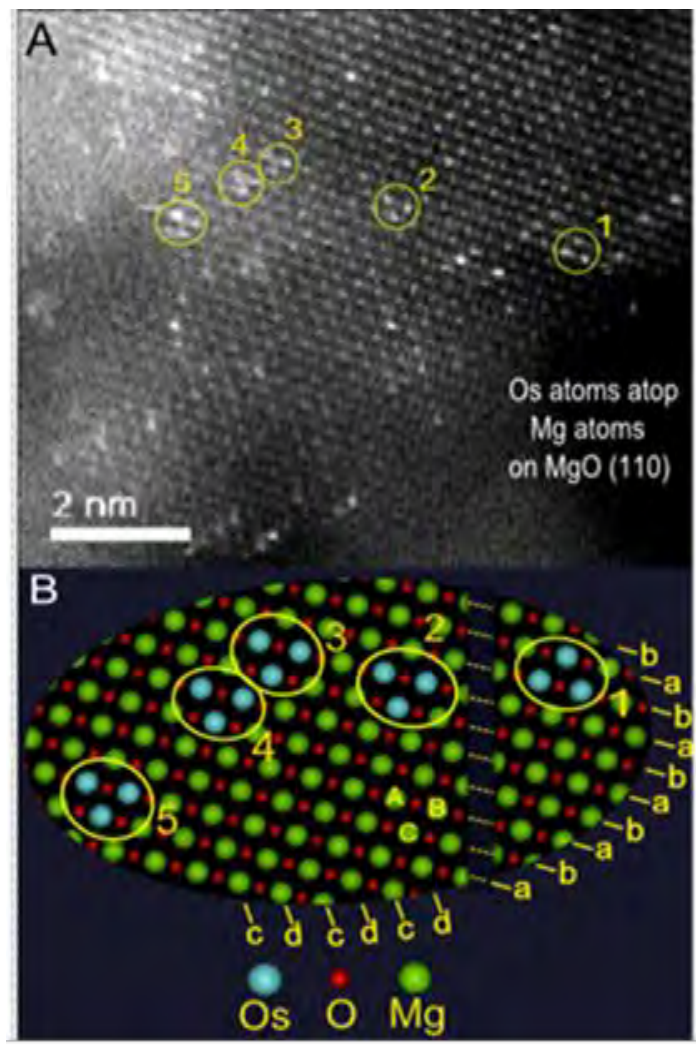

Figure 10. Aberration-corrected STEM image showing individual Os atoms and Os atoms in triosmium clusters located atop $\mathrm{Mg}$ atoms on $\mathrm{Mg}(110)$. The encircled areas show intact triosmium clusters. A is the image. $\mathrm{B}$ is a schematic representation of the image showing various orientations of the clusters on the support surface. ${ }^{110}$ 


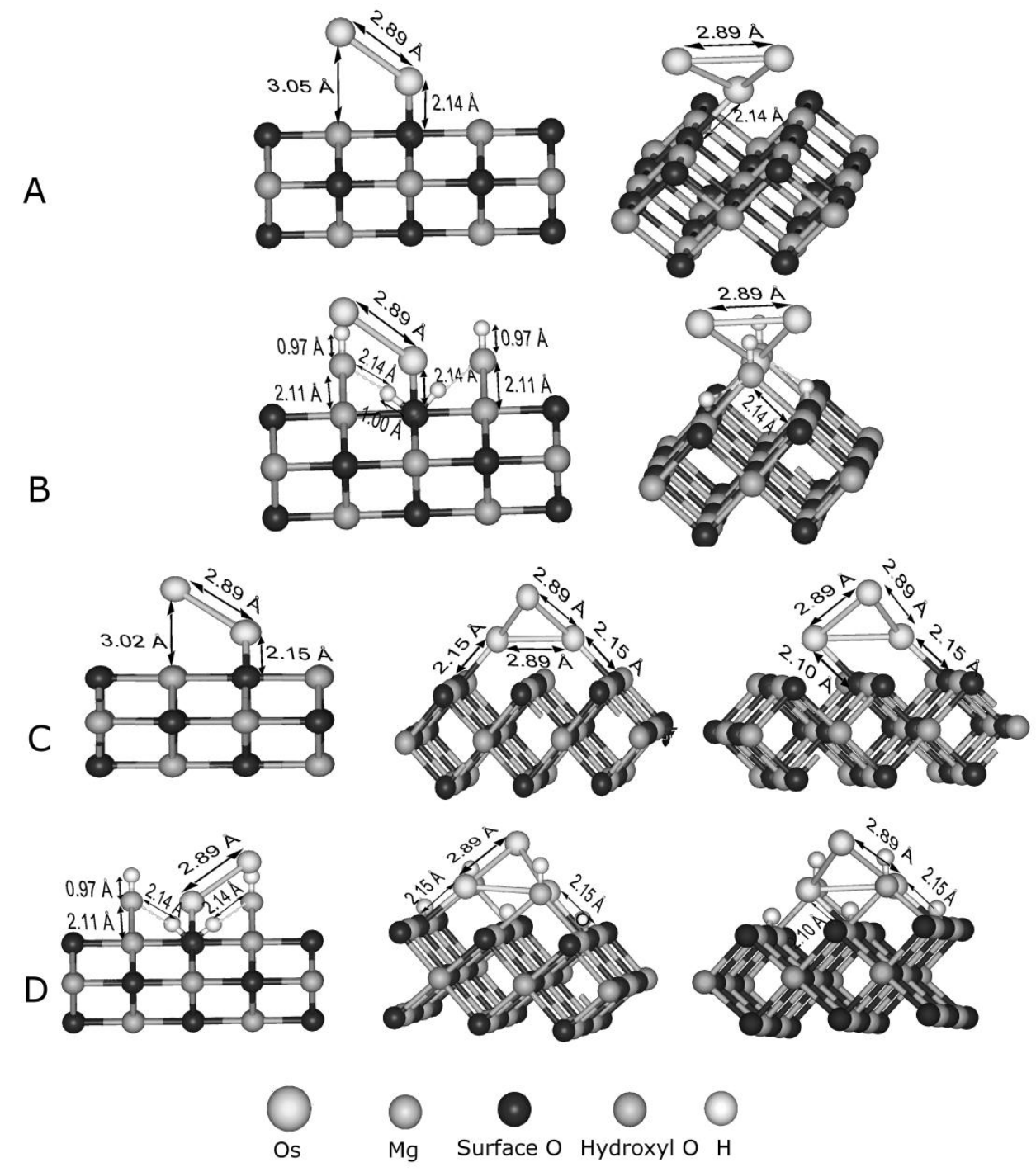

Figure 11. Two structural models of supported triosmium clusters on $\mathrm{MgO}(110)$ depicting the Os-support interactions on hydroxylated $\mathrm{MgO}$ (110) and on dehydroxylated $\mathrm{MgO}$ (110) (side and perspective views). (A) Triosmium cluster orientation I on dehydroxylated $\mathrm{MgO}(110)$. (B) Triosmium cluster orientation I on hydroxylated $\mathrm{MgO}(110)(\mathrm{C})$ Triosmium cluster orientation II, including two models for dehydroxylated $\mathrm{MgO}$ (110) and (D) triosmium cluster orientation II, including two models for hydroxylated $\mathrm{MgO}(110)$. The hydroxylated surfaces consist of hydroxyl groups on surface $\mathrm{Mg}$ atoms and protons on neighboring surface $\mathrm{O}$ atoms. The structure and bonding distances of adsorbed hydroxyls and hydrogen bonding between $\mathrm{O}$ of hydroxyl and protons on surface $\mathrm{O}$ atoms were estimated on the basis of reported density functional theory (DFT) calculations. ${ }^{\mathbf{1 1 0}}$

\subsubsection{How UTEM will Affect the Understanding of Catalysis}

Ultra-fast TEM studies will benefit our understanding of catalytic processes as follows:

- Visualizing and analyzing transient stages 
- Avoiding radiation damage, excluding artifacts caused by the electron beam which can trigger chemical reactions

- Imaging molecules on working catalysts in combination with atomic resolution of catalyst structure

- Imaging intermediates in catalytic cycles on working catalysts

- Characterizing bond breaking and bond forming in species on catalysts

- Characterizing electron transfer in species on catalysts

- Making spectroscopy specific to catalytic nanostructures and accessible during imaging.

\subsubsection{Time Scales Characteristic of Catalytic Processes}

The chemical transformations taking place in catalysis range from femtoseconds, characteristic of bond breaking and bond making in catalytic reactions, to years, characteristic of catalyst deactivation. Because the catalysts themselves participate in the bond breaking and forming, changes in their structures occur on the same time scales as those of the catalytic reactions.

A simplified, schematic representation of some of the changes that occur in solid catalysts and the representative time and length scales is shown in Figure 12. Advances in electron microscopy will allow experiments characterizing catalysts on shorter time scales, approaching those shown at left in Figure 12. Understanding the changes in catalysts on these short time scales - accompanied by atomic-resolution structural information — is one of the foremost challenges in catalysis science.

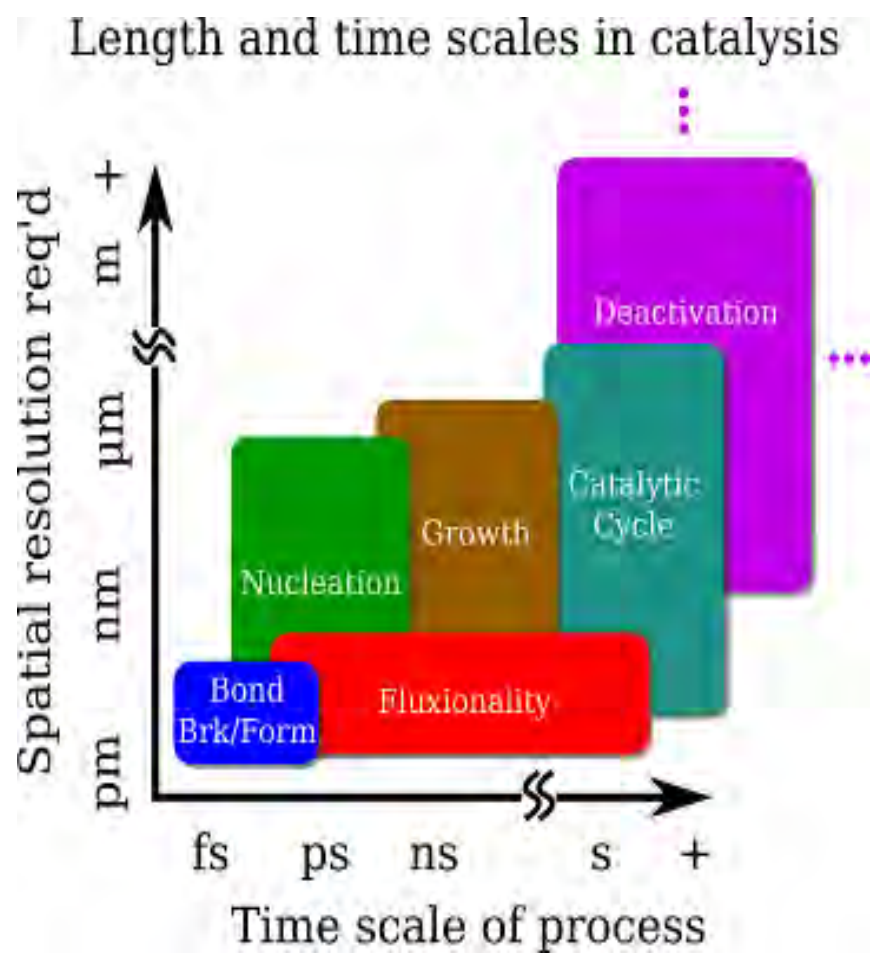

Figure 12. Approximate time scales relevant to catalysis and the corresponding UTEM spatial resolution required to image the processes. Bond breaking and formation are ultrafast (fs to ps) processes that require angstrom-scale imaging resolution. Nucleation and growth of the catalyst occur roughly on 
the ps and ns time scales, respectively, and require $\mathrm{nm}$ to micron spatial resolution. Fluxionality (changes in local morphology of the catalyst) occurs typically on time scales ranging from picoseconds to seconds. Full catalytic cycles typically occur on the order of seconds, and deactivation of catalysts takes place on time scales ranging from seconds to years.

\subsubsection{The Dynamic Nature of Catalytic Nanostructures and Catalytic Sites}

In today's research, TEM is used to investigate catalytic materials in reactive environments. This goal has been attained with maintenance of atomic resolution in imaging by employing specially designed sample holders or by modifications of conventional TEM (i.e., environmental TEM). Video-rate TEM was used to follow structural changes resulting from changes in the gas environment. For example, Figure 13 demonstrates the transformation of an iron particle in an acetylene environment into iron carbide, $\mathrm{FeC}$. This transformation occurs on a time-scale faster than the $60 \mathrm{~Hz}$ image collection rate. Although lacking the time resolution to observe precisely when this structural evolution occurred, the observations led to the identification of the active phase of iron during the growth of carbon nanotubes.
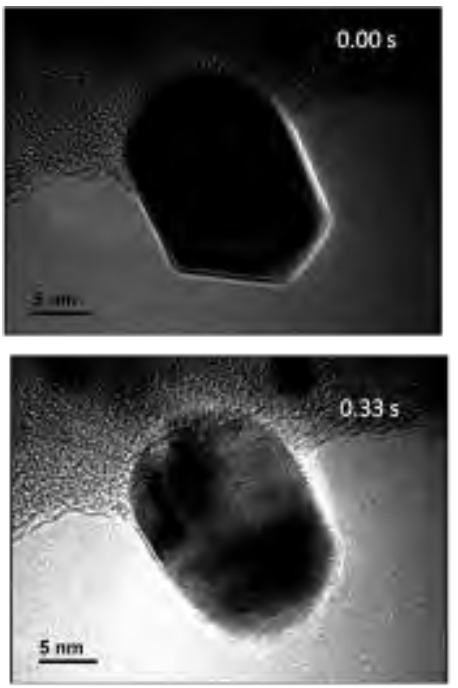
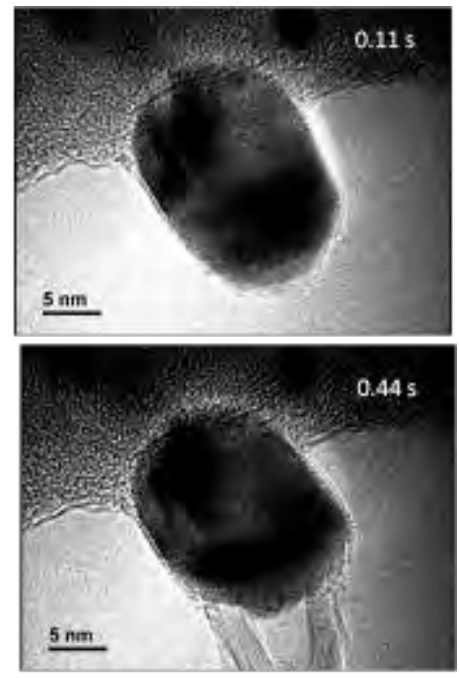

Figure 13. Static images extracted from a digital video recorded at $650^{\circ} \mathrm{C}$ as an iron particle was exposed to $10 \mathrm{~m}$ Torr of flowing acetylene with a time resolution of 9 frames $\mathrm{s}^{-1}$. The phase transformation of BCC iron into $\mathrm{Fe}_{3} \mathrm{C}$ occurred within $0.11 \mathrm{~s}$, the time resolution of the digital recording, before the growth of carbon nanotubes shown at lower right. ${ }^{111}$

In addition to composition changes of the active catalytic structure, in situ TEM also has demonstrated structural and/or morphological changes that occur during catalysis. A well-known investigation by the Topsøe group demonstrated that the faceting of a copper nanoparticle differed depending on the gas environment to which the catalyst was exposed (Figure 14). This investigation demonstrated that copper catalysts used for methanol synthesis are dynamic in nature. Furthermore, it demonstrated that both the metal and the support $(\mathrm{ZnO})$ can be imaged under in situ conditions with atomic resolution. 


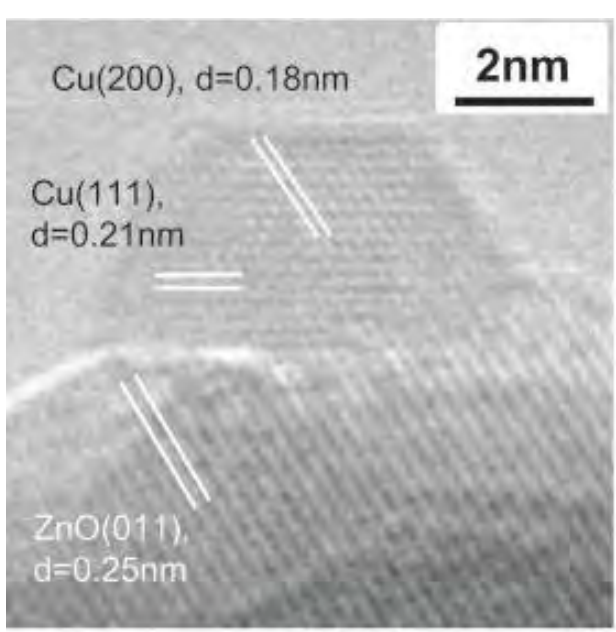

A

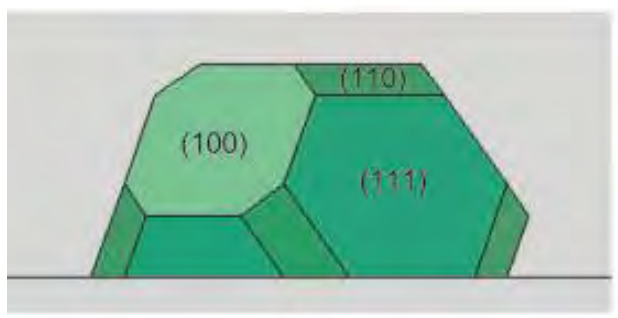

B

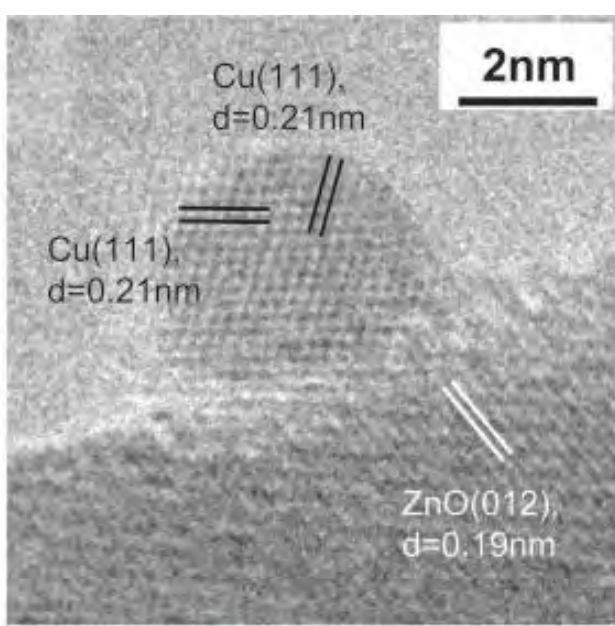

C

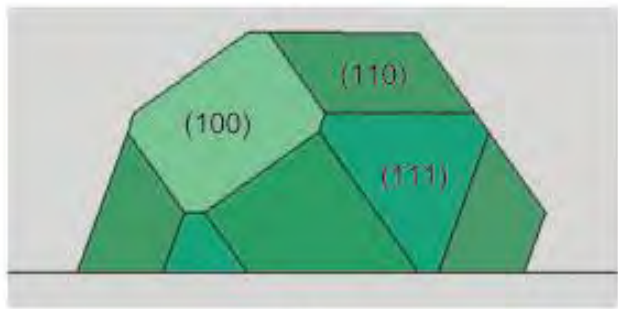

D

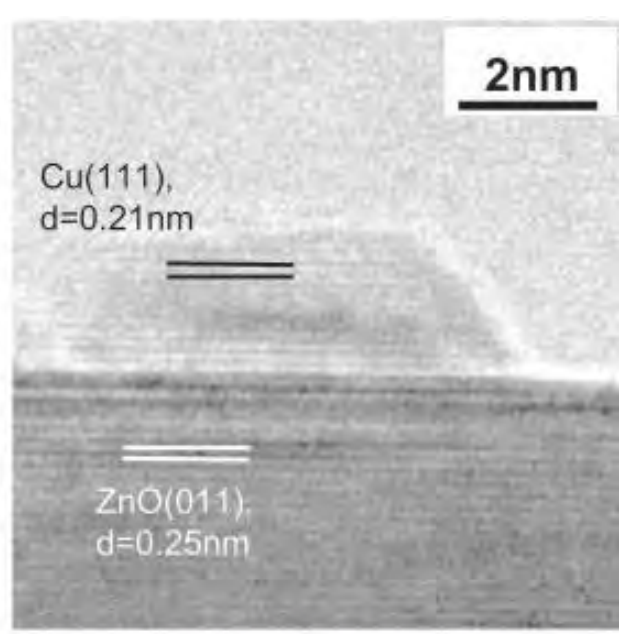

E

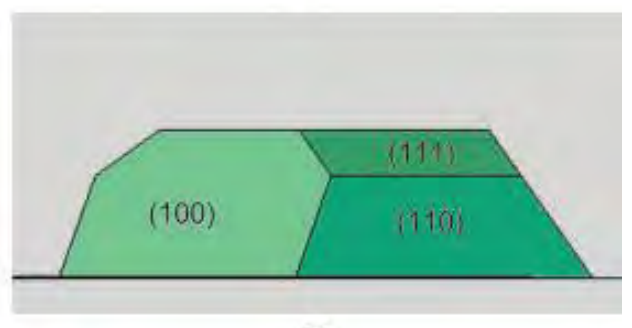

$\mathbf{F}$

Figure 14. In situ $\mathrm{TEM}$ images $(\mathrm{A}, \mathrm{C}$, and $\mathrm{E}$ ) of a $\mathrm{Cu} / \mathrm{ZnO}$ catalyst in various gas environments together with the corresponding Wulff constructions of the $\mathrm{Cu}$ nanocrystals $(\mathrm{B}, \mathrm{D}$, and $\mathrm{F}$ ). (A) The image was recorded at a pressure of $1.5 \mathrm{mbar}$ of $\mathrm{H}_{2}$ at $220^{\circ} \mathrm{C}$. The electron beam is parallel to the [011] zone axis of copper. (C) Obtained in a gas mixture of $\mathrm{H}_{2}$ and $\mathrm{H}_{2} \mathrm{O}, \mathrm{H}_{2}: \mathrm{H}_{2} \mathrm{O} 53: 1$ at a total pressure of 1.5 mbar at $220^{\circ} \mathrm{C}$. (E) Obtained in a gas mixture of $\mathrm{H}_{2}(95 \%)$ and $\mathrm{CO}(5 \%)$ at a total pressure of 5 mbar at $220^{\circ} \mathrm{C}$.

The active structures in supported catalysts are smaller than the nanoparticles depicted in Figure 13 and Figure 14 and often resemble mono- and multi-atom clusters that are used in homogeneous (or "molecular") catalysis taking place in liquids. The bond breaking and forming that catalytic clusters readily undergo is referred to as "fluxionality." There are two types of fluxionality: intrinsic and extrinsic. Intrinsic functionality depends, in large measure, on the cluster; its interaction with the support; and temperature. An illustration of intrinsic functionality is provided by molecular dynamics calculations of a 13-atom gold cluster (Figure 15). The calculations show that the cluster can access a number of configurational isomers in less than 30 ps. The results represented in Figure 15 demonstrate that changes in cluster structure take place in the absence of adsorbates and are nearly barrierless.

Depending on the environment of the cluster, fluxionality may alternatively be extrinsic. Thus, the configurational space sampled by the cluster or nanoparticle depends on its environment and adsorbed species. The fluxionality and its effect on the redistribution of electron density at the surface atoms may be markedly enhanced under catalytic reaction conditions as illustrated by calculations representing a 33-atom silver cluster during the catalytic epoxidation of propylene (Figure 16). 


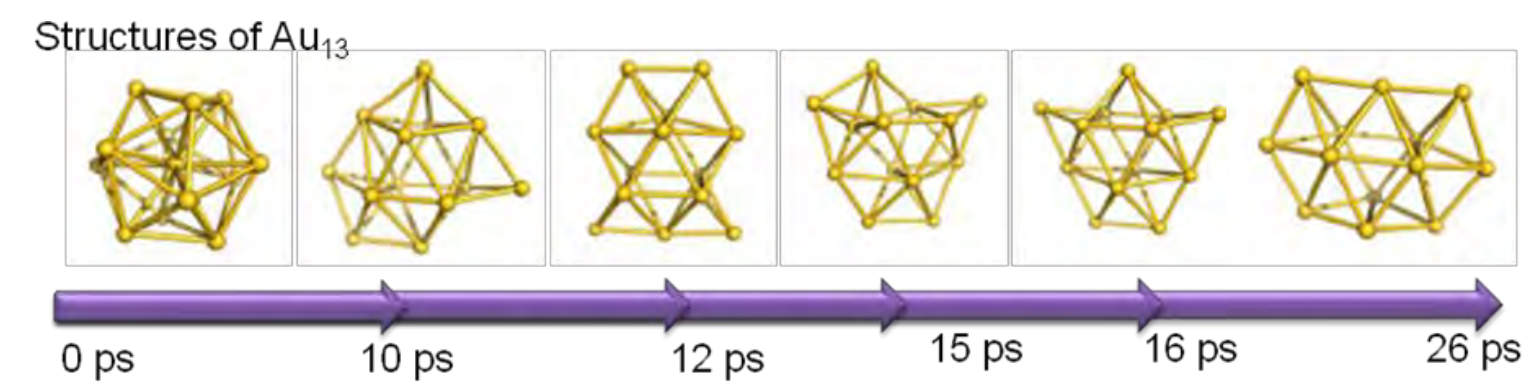

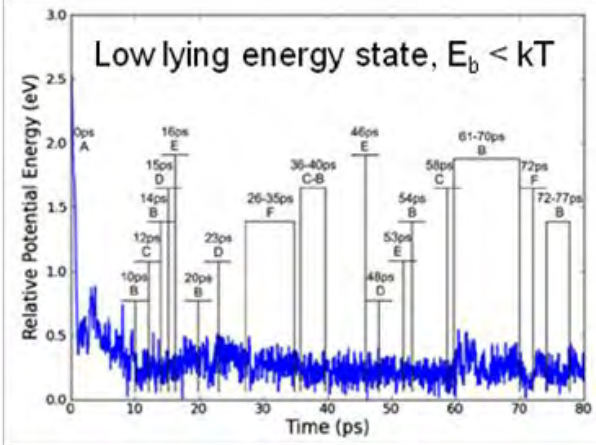

Vargas et al. 2009. Physical Review B, 80, 195421

As the number of atoms in particle increases

- Core is static and surface is fluxional

- Catalysis happens at the surface Bulk

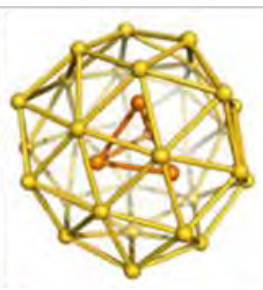

$\mathrm{d} \sim 1 \mathrm{~nm}$

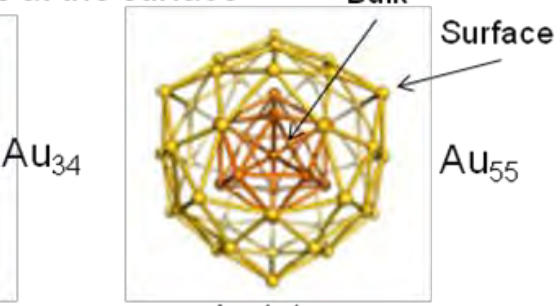

$\mathrm{d} \sim 1.4 \mathrm{~nm}$

Figure 15. Calculational characterization of fluxionality of free 13-atom gold clusters. Three-dimensional minimum energy structures of an Au13 cluster formed during the first 26 ps of a Born-Oppenheimer molecular dynamics simulation at $300 \mathrm{~K}$. Many structures were sampled numerous times within the first $80 \mathrm{ps}$ of the simulation at the sample temperature. A plot of the relative energy of these six structures demonstrates they are comparable to each other in energy, and the barriers associated with transformation between configurations are low-lying and less than $k T$ in magnitude $(2.48 \mathrm{~kJ}$ $\mathrm{mol}^{-1}$ ). For larger particles (diameter $\sim 1 \mathrm{~nm}$ ), the core (bulk) atoms are static, whereas the surface atoms are fluxional. The calculations were performed for the clusters in vacuum. It is believed such structural fluctuations will be dampened in the presence of adsorbates and supports. ${ }^{113}$

Catalytic structures are generally dynamic in nature, not just structurally but also chemically. Investigations of many catalysts for diverse reactions have shown that the true catalytic site is formed under reaction conditions-under the influence of the reactive mixture of gases or liquids in contact with the catalyst. Furthermore, the majority of the atoms that make up the catalytic entity may be uninvolved in catalysis with only a small fraction of the atoms being engaged directly. Often, the active species are metal atoms present in a low-coordination environment, such as corner or edge atoms on nanoparticles. These are the essential components of a catalyst and the ones most in need of characterization.

Bimetallic catalysts (incorporating clusters or nanoparticles with two kinds of metal atoms) often undergo surface enrichment of one component depending on the surrounding gas phase. The properties of $\mathrm{Pt} / \mathrm{Pd}$ catalysts, for example, are strongly influenced by the state of the atomic-scale mixing of the Pt and Pd atoms, whether they form alloy-like structures, core-shell nanoparticles, or separate phases. Measurements of single nanoparticles demonstrate that the presence of reactants influences the fluxional behavior of the catalytic structure, and that catalytic events are correlated with catalytic events that precede other catalytic events (Figure 17).

To understand reactivity and catalysis, it is essential to probe not only the atomic structures but also the electronic structures of these catalytic species. The goal is to one day directly image the changes in catalyst structures with atomic 
resolution while simultaneously observing the bonding in these structures on time scales relevant to catalytic reaction events.

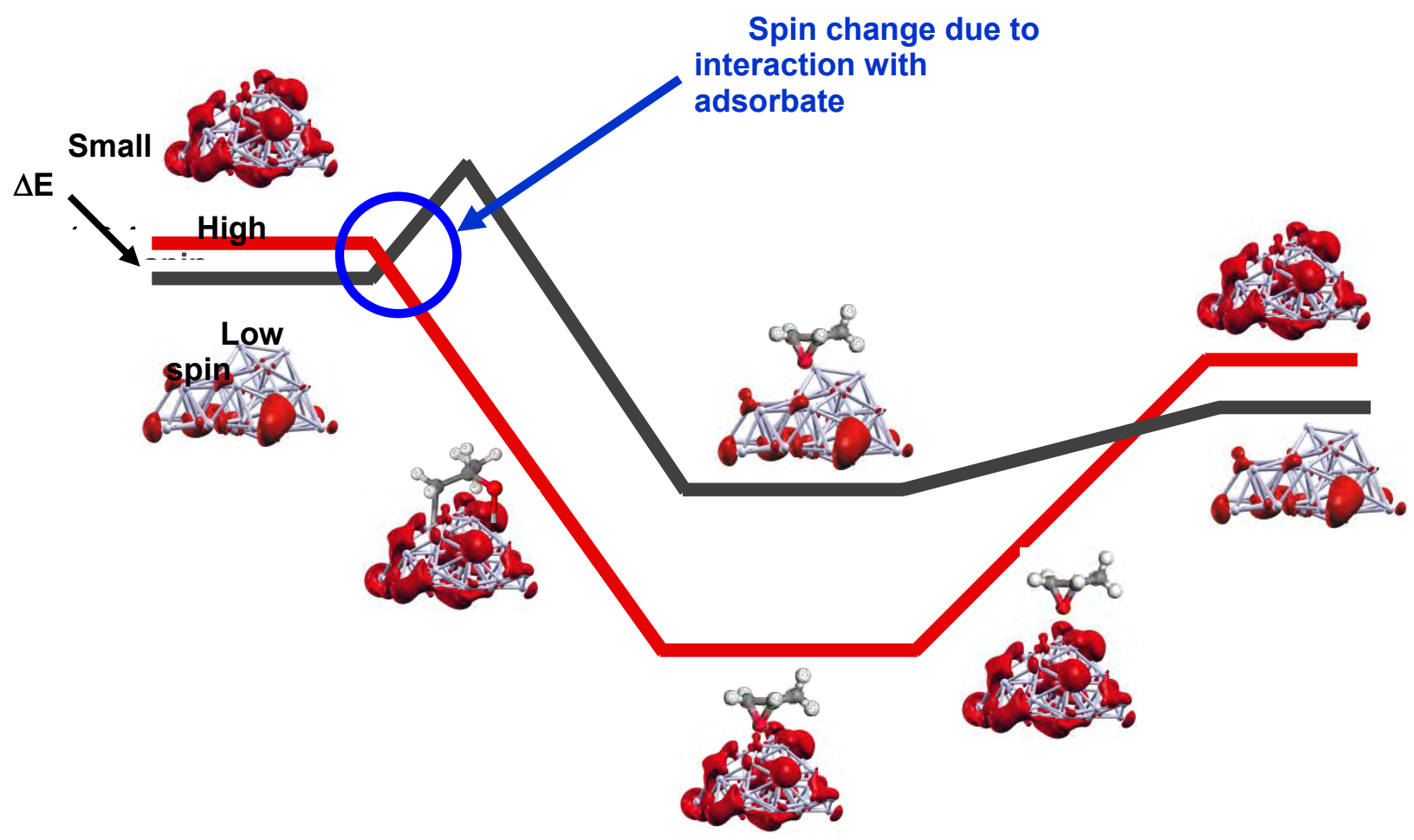

Figure 16. Schematic representation of spin-driven epoxidation reaction on Ag33. Calculations for propylene epoxidation on low-spin and high-spin $\mathrm{Ag} 33$ and of singlet and triplet $\mathrm{Ag}_{4} \mathrm{O}$ suggest that as the epoxidation reaction proceeds, a lower energy pathway is enabled via reaction on a high-spin magnetic structure that lowers the energy barrier as shown in the schematic (spin density shown in red on structures).

Because the movements of the atoms and electrons cannot be tracked in real time during catalysis, much remains to be understood about the changes that catalysts undergo as they function. Development of the UTEM is expected to make tracking of cluster and nanoparticle structures on short time scales possible. It also may become possible to use coherent nanodiffraction to examine the temporal behavior of surface relaxation during catalysis directly in an electron microscope. Spatial and temporal mapping of these structural changes is a major objective of UTEM applications in catalysis.

\subsubsection{Spectroscopy of Catalysts Used in Conjunction with TEM}

With today's TEM capabilities and proposed UTEM designs, it is not possible to observe the reactants and intermediates in catalytic reactions - imaging provides no evidence of catalysis as opposed to catalysts. This limitation leads us to a major challenge: development of methods to determine spectra of functioning catalysts along with atomic-resolution images of the catalytic species. 

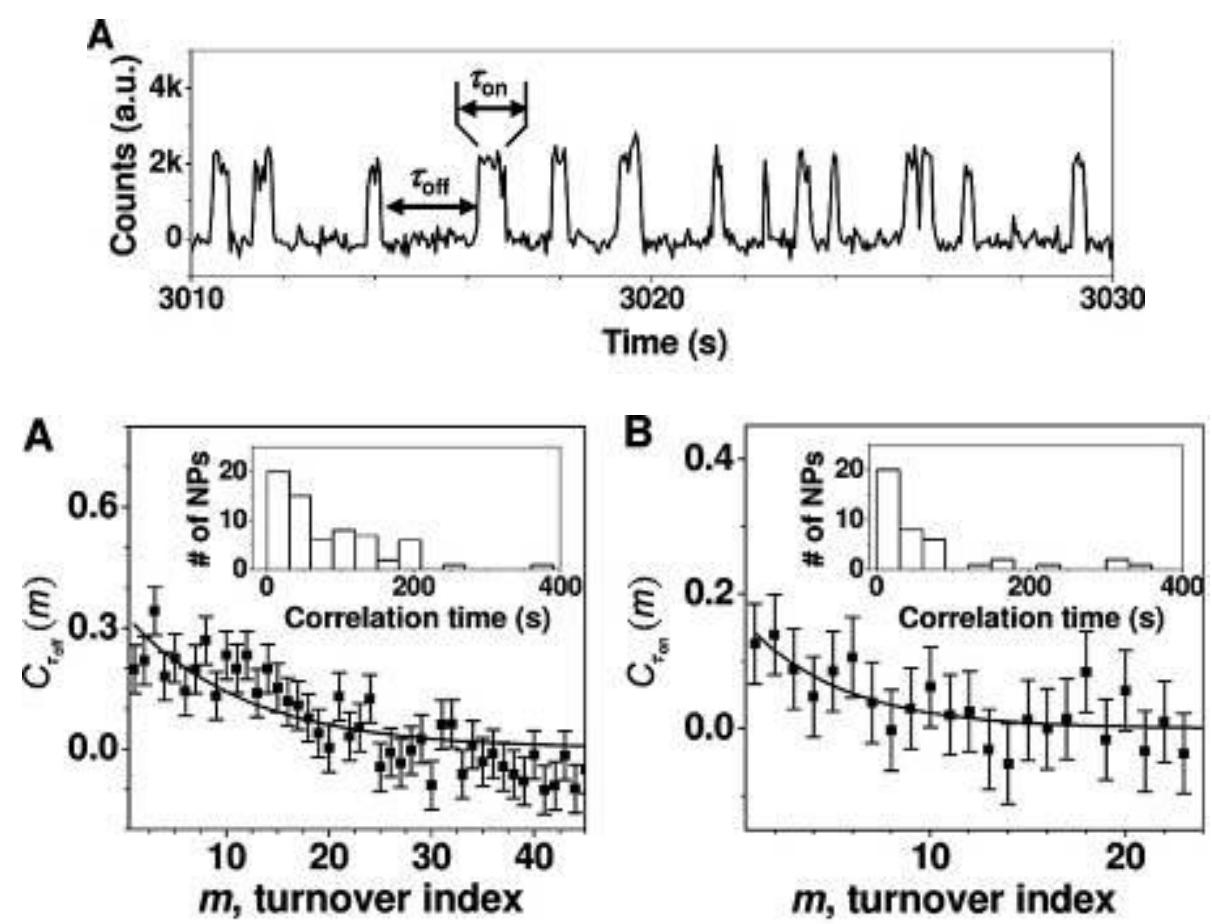

Figure 17. (Top) Single nanoparticle trajectory for the reduction of resazurin to resorufin in the presence of hydroxylamine with single-turnover resolution catalyzed by a 6-nm gold nanoparticle electrostatically bound to a glass support. The individual waiting times, $\tau_{\text {off }}$ and $\tau_{\text {on }}$, represent the time averaged single-particle rate of particle formation and rate of product dissociation, respectively. It is apparent that the individual waiting times are not identical. (Bottom) The variances in $\tau_{\text {on }}$ and $\tau_{\text {off }}$ are believed to be associated with the fluxional behavior of the underlying surface atoms of the gold nanoparticle. For each nanoparticle, the temporal variation of its rate of turnover (catalysis) has contributions from the reaction rate changes in $\tau_{\text {off }}$ and $\tau_{\text {on }}$. These two contributions can be evaluated for each nanoparticle by extracting the sequences of individual $\tau_{\text {off }}$ and $\tau_{\text {on }}$ from its turnover trajectory and analyzing their autocorrelation functions $C_{\tau}(m)=$ $<\Delta \tau(0) \Delta \tau(m) / \Delta \tau^{2}>$, where $\tau$ is either $\tau_{\text {off }}$ or $\tau_{\text {on }}, m$ is the turnover index number in the sequences, and $\Delta \tau(m)=\tau(m)-<\tau>$. In the presence of activity fluctuations, $C_{\tau}(m) \geq 0$ and shows exponential decay behavior. The time constant associated with this decay is the activity fluctuation correlation time. The insets demonstrate that individual particles in a seemingly homogeneous population of 9$\mathrm{nm}$ gold nanoparticles have diverse activity fluctuation correlation times, indicating that individual nanoparticles can have very different surface restructuring time scales. The correlation between restructuring rates and activity fluctuations in catalysis is not direct but is inferred because the fluxional nature of the gold nanoparticles cannot be observed directly. Although this inference is consistent with our picture of the catalytic surface as a dynamic entity, UTEM observations during catalysis offer the prospect of enabling direct measurements of these fluctuation rates and correlations of these rates with fluctuations in either $\tau_{\text {off }}$ or $\tau_{\text {on. }}{ }^{114}$

EDXS and EELS are the two basic spectroscopic methods used in conjunction with TEM to probe the chemical composition of a sample. Traditionally, EDXS provides a fast capability for capturing elemental information with a detection limit of about $0.5 \mathrm{wt} \%$ and spatial resolution on the nanometer scale. With recent advances in aberration correction techniques in TEM and STEM and the invention of new detector configurations, elemental identification with EDXS has been demonstrated to reach a spatial resolution of a single atomic column. However, a consequence of the high 
spatial resolution is damage to the sample caused by the electron beam. Although the EDXS technique has been demonstrated single atomic column resolution, it has been limited, essentially, to ideal samples, such as single crystals of $\mathrm{SrTiO}_{3}$. For more complex and realistic catalyst samples, the attainable chemical spatial resolution is compromised. And, there currently is no known way to monitor the time dependence of the chemical compositional evolution of catalysts on the time scale of this process.

EELS captures information related to the inelastic scattering of electrons by a sample, such as the excitation of a core electron to an unoccupied energy level, which is a process governed by the quantum dipole selection rule. As x-ray absorption processes can be divided into x-ray absorption near edge structure (XANES) and extended x-ray absorption fine structure (EXAFS), EELS also can be divided into two sections: 1) energy loss near edge structure (ELNES) and 2) extended electron energy loss fine structure (EXELFS). The information obtainable with XANES can be obtained similarly by ELNES. The ELNES feature maps out the unoccupied density of states with a specific angular momentum quantum number. For example, if a $K$-edge EELS spectrum is recorded, it will give the density of states related to unoccupied p-states. ELNES can be used to extract information related to the local bonding/coordination environment and valence state. As a result of recent advances in aberration-corrected TEM and STEM, ELNES now can reach the spatial resolution of a single atomic column.

A fundamental challenge is to extract atomic-scale structural information from experimental ELNES. Currently, a trialand-error approach is needed. New fitting methods in the context of DFT calculations may be sought to improve the technique.

Spectroscopic probes found within TEM, such are EELS, are amenable to in situ methods. For example, data showing EELS results characterizing the redox reactions of cerium-containing nanoparticles are shown in Figure 18.

\section{$\mathrm{M}_{4,5}$ edge in pure $\mathrm{CeO}_{2}$ at RT in 2 Torr $\mathbf{H}_{2}$}

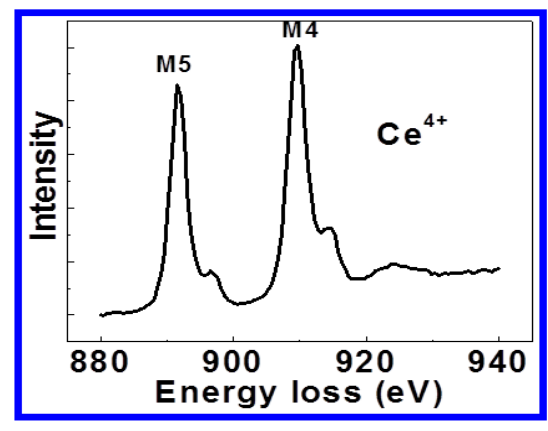

Oxidized

\section{$\mathrm{M}_{4,5}$ edge in pure $\mathrm{CeO}_{2}$ at $700^{\circ} \mathrm{C}$ in 2 Torr $\mathrm{H}_{2}$}

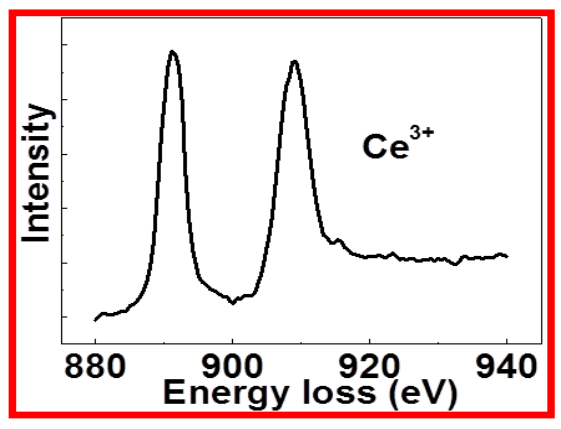

Reduced

\section{Ce oxidation state and white-line ratio}

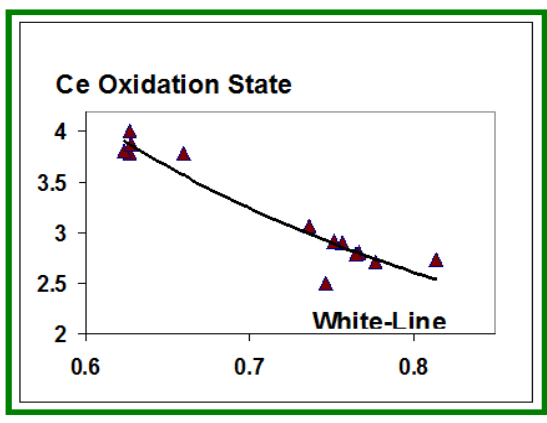

$A=1.81 R^{-1.627}$

Figure 18. In situ detection of redox reactivity of cerium by EELS: Ce $\mathrm{M}_{4,5}$ white line intensities in electron energy loss spectra are used to detect local changes in the cerium oxidation state in $\mathrm{CeO}_{2}$ nanoparticles during redox reactions. The intensity of the $C e M_{5}$ increases relative to $M_{4}$ during reduction and can be plotted against the oxidation state to obtain oxidation state directly from the white-line ratio. The technique can be used for doped ceria and can be extended to other transition metal oxides. 115 
Although EELS can provide chemical and electronic information at high spatial resolutions, the time resolution still is not adequate for many problems related to catalysis research. EELS is a method expected to be suitable for dynamic ultrafast measurement. At least, the signal in the low loss area is high enough to provide information about band gap, conductivity (plasmon peak), and elemental and chemical information of elements that have EELS transitions below $\sim 300 \mathrm{eV}$.

Chemical reactions that modify the binding state of elements, such as $\mathrm{C}, \mathrm{Si}, \mathrm{Al}$, and $\mathrm{Fe}$, could be investigated with time resolution in the picosecond range.

Advances in EELS detector technology and in development of correlative spectroscopic techniques that can be integrated into the UTEM are needed. These may include techniques such as first-order spectroscopies (e.g., infrared or Ramanboth normal and resonance) or higher order, nonlinear spectroscopy (e.g., sum frequency generation). Methods capable of single-molecule sensitivity also are highly desirable.

Any of these techniques must be characterized with a time resolution commensurate with femto/picosecond atomicresolution imaging. Spectroscopic investigations of functioning catalysts can provide inspiration for the pursuit of combined microscopy-spectroscopy measurements within the UTEM. However, the two techniques must have the same sensitivity because the mass of a sample that can be charged to the UTEM is limited. Furthermore, the techniques must provide complementary and/or orthogonal information on the UTEM time scale.

This last requirement is a pump-probe scheme to achieve the ps - fs time scales. If the measurement can be done before radiation damage occurs, UTEM affords the ability to observe the undisturbed catalytic reaction. This condition should be fulfilled by femtosecond exposure for EELS spectroscopy and imaging on a nanometer scale.

Ultimately, we envision the UTEM will enable not only simultaneous spatial and temporal monitoring of the catalytic species with atomic resolution of their structures but also monitoring of the adsorbates on them. This capability could be accomplished with advances in EELS detector technology or the combination of correlative techniques (microscopy and a host of spectroscopic techniques).

A complementary method is x-ray scattering, illustrated by data from an investigation of size-selected silver nanoparticles demonstrating a time- and temperature-dependent evolution of the morphology as shown in Figure 19.

\subsubsection{Statistical Assessment of TEM Data and the Motivation for Investigation of Well-defined Model Catalysts}

Industrial catalysts usually consist of structurally, morphologically, and chemically complex nanoscale components. For example, the spatial and size distributions of nanoparticles in a supported metal catalyst are frequently not uniform and can vary significantly from one region to another. The composition of bi- and multi-component nanoparticles also may be spatially inhomogeneous. Because of this complexity, it is a formidable task to fully characterize and understand the nature of practical industrial catalysts.

As with other ultra-high resolution imaging techniques, electron microscopy yielding atomic-resolution images, diffraction patterns, or spectra provides unparalleled spatial resolution of the area of interest, but it may lack statistical value or relevance to understanding the catalyst's performance. To assure the relevance of electron microscopy data for determining structure-catalytic property relationships and guiding development of optimized catalyst synthesis protocols, a robust sampling and data acquisition protocol must be followed. 

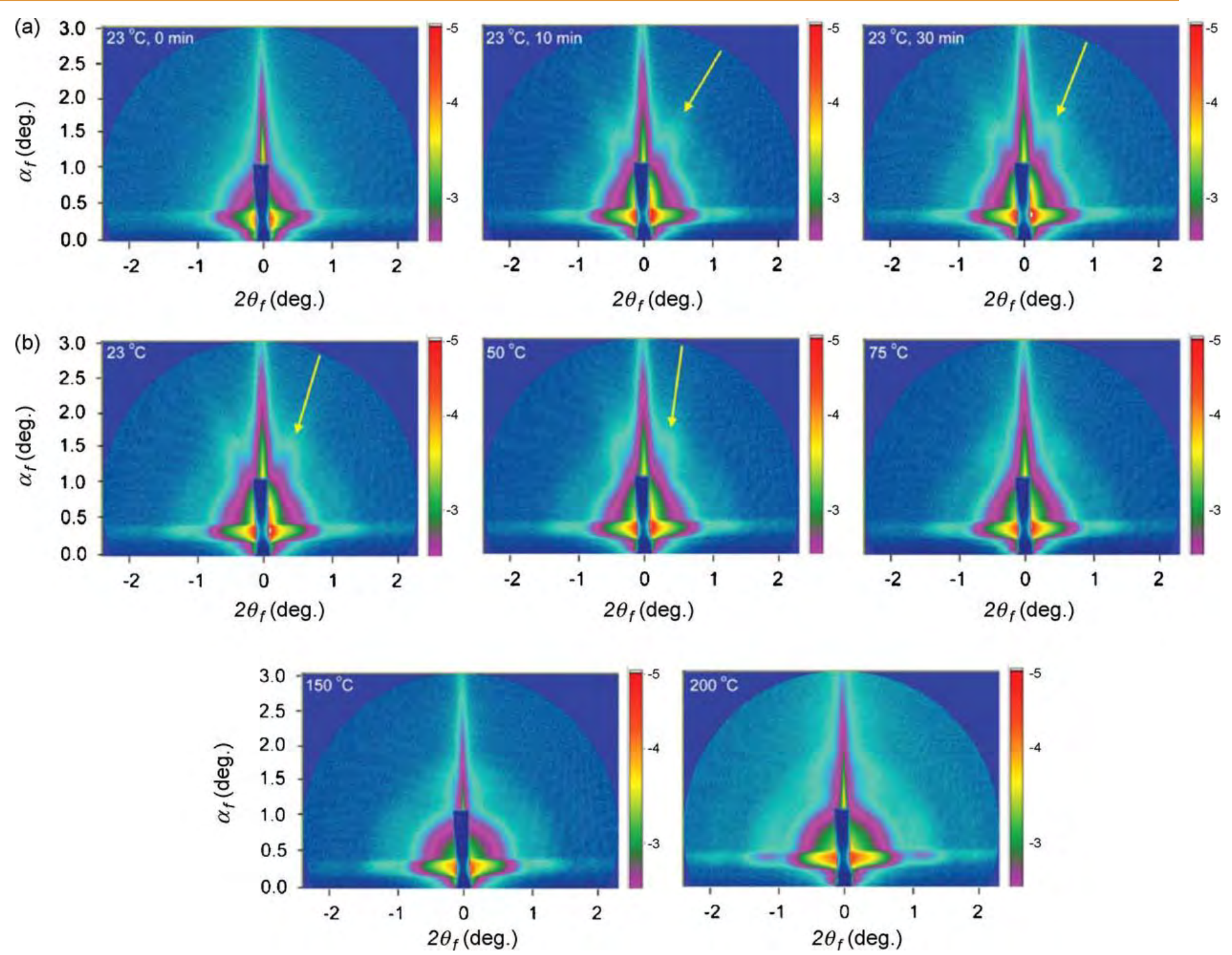

Figure 19. Grazing-incidence small-angle x-ray scattering (GISAXS) images of $~ 9$-nm silver particles (a) at room temperature, from left to right: image recorded at the time of the inlet of the propyleneoxygen mixture, after $10 \mathrm{~min}$ of exposure and after $30 \mathrm{~min}$ of exposure. (b) Change of the scattering pattern during ramping of the temperature from 23 to $200^{\circ} \mathrm{C}$. The arrows indicate the angle of the most intense component of the directional scattering, which is indicative of changes in the faceting of the particles. The evolving asymmetry of the scattering pattern indicates the change of particle shape from oblate-like to spherical. ${ }^{22}$

For investigations of practical catalysts, which usually have non-uniform spatial distributions of the nanoscale catalytic components, many images of various regions must be acquired to guarantee statistically meaningful conclusions. The validity of such an approach can be checked if one can correlate the change of the experimentally acquired data with the change of catalyst synthesis parameters or in catalytic performance. A good correlation provides more confidence in the useful insights obtained by analyzing many high-resolution images, diffraction patterns, or spectra. 
For highly inhomogeneous practical catalyst samples, a simple model system suitable for TEM investigation must be extracted because the analysis of thousands of different nanoparticles cannot be achieved in a reasonable amount of time. If the model system has been chosen well, it should be possible to derive conclusions for the practical system. Selection of appropriate model catalysts, such as nanoparticles with controlled sizes and/or shapes, can greatly reduce the uncertainty of high-resolution experimental results. Work with such samples is an essential part of the necessary effort. An integrated microscopy approach (including systematic imaging from very low magnifications with SEM to mid-magnifications with TEM and very high magnifications with atomic-resolution electron microscopes) may help to alleviate the problems associated with sampling issues. Accompanying x-ray investigations also should improve the statistical value of the TEM results. Model catalysts (sub-nanometer clusters) of well-defined size and composition can be prepared with atomic precision or with extremely narrow size distributions. Such model catalysts have several advantages, allowing for 1) exact determination of size effects in catalysis under realistic reaction conditions; 2) exactly matching theoretical calculations and modeling; and, in the case of UTEM investigations envisioned in this report, 3) assurance that all the data obtained in the microscope (e.g., determination of catalytic activity and magnetic moments) are precisely size-specific. The latter point alleviates the need for large-scale and time-consuming experiments that would otherwise be necessary to gain statistically satisfactory data.

\subsubsection{Additional Challenges and Opportunities}

In the quest for ultrafast electron microscopy, it is important to realize that as time resolution is increased, spatial resolution suffers. An essential question the catalysis community needs to address is how far down that the temporal axis can we go without losing atomic resolution. Atomic resolution, especially for characterization of catalysts under working conditions, is crucial to understanding catalyst structure-function relationships. If we envision observation of bondbreaking and bond-making events, electron transfers, or movements of charges in the future, will we be able to image the active sites simultaneously.

Time resolution in the pico- and femto-second range allows access to many phenomena that are inherent not just to catalysis but to catalyst synthesis and catalyst modification under the influence of pretreatments, application of promoters, etc. The phenomena include nucleation and particle growth; defect dynamics, including mobility and volatilization of catalyst components; among others.

Combining UTEM technology with in situ cell design for both gas and liquid environments will enable us to understand varied phenomena, including solvation of solid surfaces and catalyst dissolution, leaching, and redeposition. Some specific applications may include the influence of aqueous environments on catalyst structure and dynamics, which is applicable to conversion of biomass in the presence of aqueous media, or the formation of chiral metal sites for fine chemicals synthesis. An obvious opportunity for application of liquid cells and UTEM is electrocatalysis.

Another major challenge involves pump-probe methods. They have limited application in the investigation of catalysis because the laser pump is non-innocent and can change the catalyst. For catalytic science, the pump in UTEM represents a particular challenge on many fronts. To probe phenomena on more than one catalytic structure without having to load a new sample, the probe should be focused down to the dimensions of a single nanoparticle. Catalysts are structurally complex and heterogeneous in nature, so the ability to confine the pump and the system response to a small subset of the catalytic structure population is crucial to understanding catalytic behavior and for allowing repeated measurements to build statistically significant numbers of events. To this end, it is recommended that model catalysts such as those mentioned above be employed. Another possibility is to use the concept developed by Diaz et al. ${ }^{116}$ to determine the heat of adsorption of molecules on thin single crystals (via microcalorimetry) by measuring blackbody emission for catalyst bodies. Although appropriate for thermally uniform materials (i.e., those described by a single heat capacity and thermal 
conductivity values), the measurement of blackbody emission most likely will be insufficient for a material such as a set of catalytic nanoparticles on a support. Advances in heat transfer modeling and understanding of laser-materials interactions are required to accurately assess the temperature in heterogeneous catalysts after laser perturbation.

The challenge of determining the temperature of a catalytic nanoparticle during treatment or a catalytic reaction might be addressed by inclusion of nanoscale thermometers (quantum dots) in the samples. Individual quantum dots of CdSe are capable of sensing temperature variations and reporting temperature changes remotely through optical readout.

Another approach for measuring temperature demonstrated under catalytically relevant conditions (at elevated temperatures and pressures) is using the response of the localized surface plasmons to changes in environmental conditions. This procedure limits the catalytic phase to coinage metals that have appreciable surface plasmon resonances. The plasmon response of a gold nanoparticle during catalysis can be used as an optical nanocalorimeter, which should allow the determination of temperature through temperature-dependent shifts in the plasmon peak position.

The foregoing discussion suggests that a new scenario with regard to perturbation of a catalytic material in UTEM needs to be developed. The most appropriate perturbation is one that disrupts the composition of the gas phase during previously steady-state catalysis. The development of rapid- or step-scan spectroscopy has been aided by the use of mechanically pulsed valves with reproducible millisecond response times or the creation of reactants via photolabile generation with microsecond time resolutions. In the case of UTEM, the technology for introducing a perturbation to the gas phase is much more challenging. There is no valve technology that has a response time on the order of femtosecond to picosecond. Indeed, the fastest mechanical valve is many orders of magnitude slower than that required for UTEM. The second method that has been used - photogeneration of reactive species from a photolabile host —is limited by the photochemistry. In this case, time resolution on the nanosecond scale is possible if the appropriate photolabile molecule is realized. This challenge represents a significant hurdle in the application of UTEM to catalysts and catalysis characterizations.

\subsubsection{Connecting Experimental Results with Theory}

As highlighted in the 2007 DOE-BES report, "Basic Research Needs: Catalysis for Energy", ${ }^{107}$ theory and computation will play an increasingly important role in the development of new catalytic processes by providing detailed mechanistic insights, interpretations of experimental phenomena, and eventual prediction of improved catalysts. The computational design of practical catalysts requires the ability to predict, at the molecular level, the detailed behavior of large, complex molecules and solid structures together with their reaction environments.

As previously illustrated in Figure 12, a range of diverse time and length scales are important in catalysis, and these are represented by different levels of theoretical models. Ultrafast processes, such as electronically excited bond breaking, occur on the time-scale of electron/nuclear motion (fs to ps) and typically are modeled by dynamical electron theories such as time-dependent DFT (TD-DFT). Chemical reactions (ps scale events) are modeled by electron structure methods coupled with either dynamics or transition state theory, while the more complex reaction kinetics of the entire catalytic process are modeled by kinetics models. It is critical to realize that a catalytic process is a set of complex multiscale phenomena often involving as many as thousands of chemical reactions with global macroscopic kinetics that depend on, for example, the barriers of individual chemical reactions and their dependence on local reaction environments, thermodynamic conditions and constraints of temperature and pressure, mass and heat transport, and how the kinetics of the individual elementary reaction steps are coupled to each other. Although computational models exist for simulations at any one of these time and length scales, they require extensive knowledge of the catalyst for construction of a realistic model of its structure and chemical reactivity. 
In this context, UTEM measurements can function in a complementary way to theoretical simulations to meet the following goals:

- As noted, electron microscopy methods already provide realistic structural models of some catalysts under working conditions. This information is indispensable for the construction of models with which simulations of catalytic reactions (or complex coupled kinetics and reaction networks) can be performed.

- Less obvious but equally valuable is the ability of using time-resolved electron microscopy measurements for estimating species concentrations of catalyst structures and kinetics of chemical reactions. As with time-resolved scanning tunneling microscopy (STM) images, this information can be used to provide quantitative information about chemical equilibrium and reaction kinetics under catalytic reaction conditions. This information can be used to benchmark theoretical models of catalyst transformations. On the other hand, theoretical tools may be used to provide interpretations of electron microscopy data, especially when the observed transformations are induced by adsorbates not visible by the imaging technique.

- Spectroscopic modeling of experimental observables (such as EELS spectra or vibrational spectra) can provide further confirmation of structural models and aid in data interpretation.

- Combination of short-time dynamics (e.g., bond breaking) and long-time dynamics (e.g., catalyst-structure transformations) can be used to help inform the construction of multi-scale kinetics models that take account of fluctuations across a wide range of temporal and length scales. Such models are at the forefront of computational catalysis research, and electron microscopy methods are in a unique position to provide the detailed information needed to elucidate what is needed for their construction.

\subsubsection{Observation of Highly Fluxional Rhodium Catalysts for the Dehydration of Amino Boranes}

The identification of active catalytic species in conjunction with the characterization of catalytic reaction pathways is an ongoing challenge in experimental and computational chemistry. Spectroscopic methods that can be used to identify and follow the fate of the key intermediates formed during a catalytic reaction help elucidate mechanistic reaction pathways. However, ensemble average methods of characterization, even with catalysts under working conditions, often are not definitive enough to provide the atomic-level detail needed to hypothesize a mechanism or to characterize time-dependent phenomena such as the dynamic motion of the catalyst structure. Time-resolved methods can help determine the degree of fluxionality in a catalyst - information that can be compared with results of detailed theoretical modeling.

As an illustrative example (Figure 20), XAFS experiments characterizing the working catalyst in the dehydration of dimethylaminoborane identified the presence of a Rh4 cluster species as an active catalyst and also provided a multitude of possible static structures that may be compatible with the XAFS signal. ${ }^{117}$ Using $a b$ initio molecular dynamics (AIMD) and XAFS modeling, it was possible to identify the structure of the catalyst's resting state. It also showed the proposed $\mathrm{Rh}_{4}$ species are highly fluxional. From this proposed resting state, a reaction mechanism for the extraction of $\mathrm{H}_{2}$ from $\mathrm{BH}_{3} \mathrm{NMe}_{2} \mathrm{H}$ was inferred.

A daunting challenge in linking theory and UTEM experiments is modeling the initial response of the system to the laserpulse trigger. In contrast to typical catalytic events, the response of the system to the pump is a non-thermodynamic ultrafast process involving femtosecond-scale electron dynamics and picosecond/sub-picosecond nuclear dynamics in the 
local vicinity of an active site. For example, an intense optical trigger tuned to the adsorbate might involve nonlinear valence excitations in the molecule, charge transfer to and/or from the catalyst, and photo-induced bond breaking.

Real-time simulations of the combined electronic-nuclear dynamics are a powerful approach to this problem. Both electron density and nuclear motion are explicitly propagated in time under the influence of the laser pump and for a short time after by using TDDFT. Real-time simulations are a natural complement to UTEM imaging as they offer direct insight into the mechanism of pump excitation and are excellent tools for interpreting and understanding the resulting dynamics. Moreover, the outputs of these simulations can serve as starting points for subsequent simulations of catalytic bond breaking and formation, fluxionality of the catalyst, etc. - - a first step toward bridging simulations from the ultrafast to catalytic time scales.

(a)

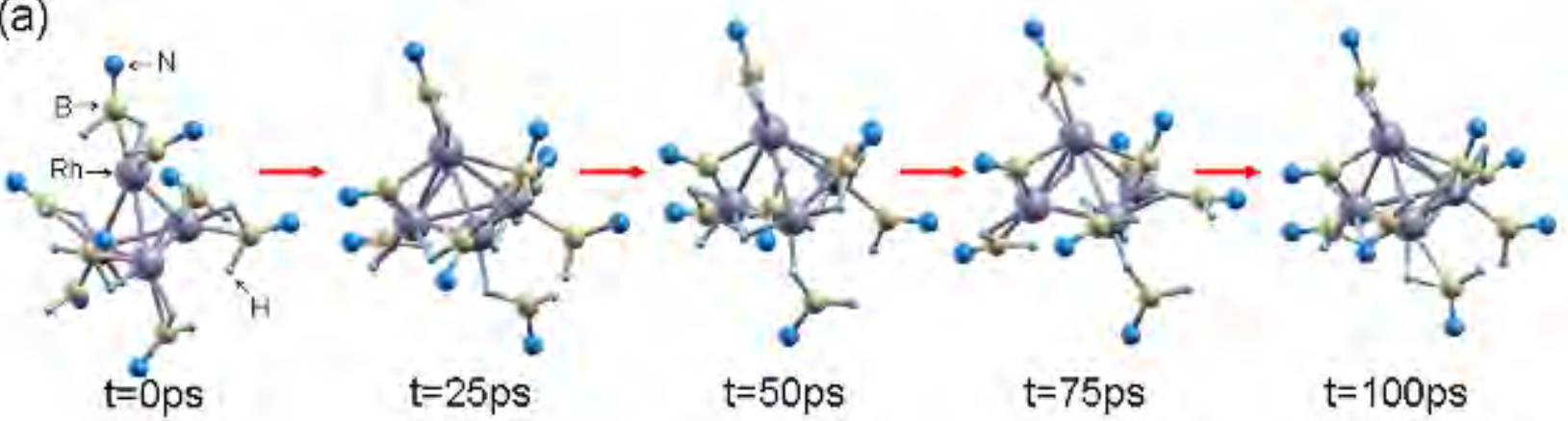

(b)

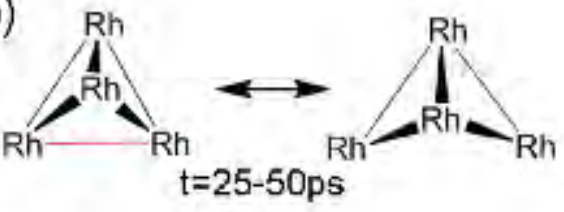

(c)

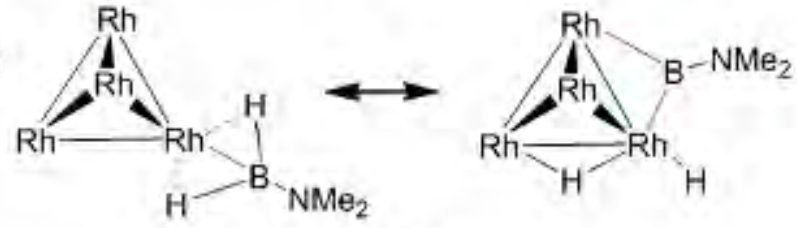

$t=0-25 p s$

(d)

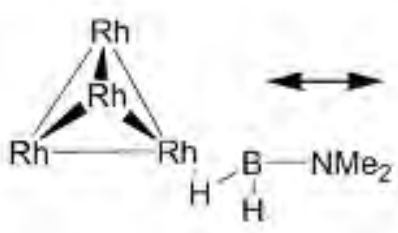

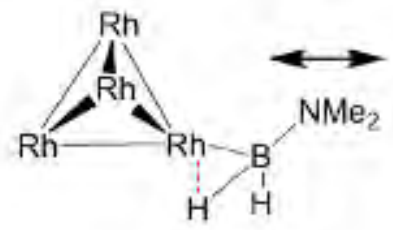

$\mathrm{t}=0-25 \mathrm{ps}$

Figure 20. Fluxionality of a catalyst resting state observed in short-time AIMD simulation: (a) structure of $\mathrm{Rh}_{4}\left(\mathrm{BH}_{2} \mathrm{NMe}_{2}\right)_{8}{ }^{2+}\left(3^{\prime} \mathrm{H}_{0}{ }^{2+}\right)$ at $300 \mathrm{~K}$ shown at 25 ps intervals. Methyl groups have been subtracted for clarity. The atom colors are as follows: N, blue; B, yellow; H, light blue; and Rh, gray. Key fluxional processes evident in the AIMD trajectory are illustrated schematically: (b) Rh4 cluster isomerization, (c) terminal-to-bridge ligand migration, and (d) terminal ligand binding isomerization and hydride transfer. The times indicated in parentheses in (b)-(d) are indicative of where this process may be seen in the configurations given in the molecular dynamics configurations presented in (a) ${ }^{118}$. 


\subsection{Materials Science}

\subsubsection{Introduction}

Materials dramatically enhance the human experience through their diverse functionalities. Early functionalities included static features such as strength, durability, and thermal insulation. More recently, sophisticated dynamic functionalities have become dominant, such as digital switching for high-speed computation, conversion of sunlight into electricity and fuel, and catalytic capture of toxic combustion products in automobiles. We have developed static functionalities by relating them to the structure of atoms and electrons observed in real space with remarkable spatial resolution in electron microscopy. Dynamic functionalities have significantly greater potential than static functionalities because they transform charge, energy, spin, and chemical species from one form to another, enabling our traditional and alternative energy production and use chains and our chemical production chains. Biology has made enormous use of dynamic functionality, producing living organisms adapted to every environment, from undersea hydrothermal vents to arid deserts to frozen tundras. One prime scientific challenge and opportunity is duplicating the diversity and complexity of biological dynamic functionality in equally complex inorganic architectures. Biology provides the proof of principle, the range of inorganic materials combinations and opportunities is endless, and we are just beginning this promising and exciting new enterprise.

However, one key element is needed to understand, design, and control dynamic functionality - the ability to observe ultrafast dynamic behavior with molecular and atomic resolution. For example, photovoltaics convert one photon to one electron in a sequential process. To design and control this process, we must be able to observe each step: the excitation of an electron by a photon from the conduction to the valence band in a semiconductor, the separation of the electron from the hole it left behind in a p-n junction, and the transport of the electrons and holes in opposite directions without loss due to recombination. Electronic excitation and recombination are ultrafast processes involving a single electron, requiring simultaneous high spatial and temporal resolution to explore, understand, and control.

The time scales of atomic and electronic phenomena in materials fall into three broad categories as illustrated in Figure 21 1) polarization reversal in magnetic, ferroelectric, and multiferroic systems ${ }^{119}$; 2) atomic rearrangements depending on phonon excitation; and 3) electronic rearrangements depending on electron excitation. The fastest and most challenging time-scale is for pure electronic excitations, which, in isolated atoms, take 10-100 attoseconds to complete. This short time-scale is determined by the high speed of light, the small size of an atom, low mass of the electron, and simplicity of isolated one-electron excitations. In molecules and solids, where electrons are shared among atoms and excitations involve simultaneous rearrangement of many electrons, the time-scale can be longer.

Atomic rearrangements often are linked to electronic excitations as in chemical reactions among molecules and phase transitions in solids. Atomic motions are described by phonons, the collective excitation of many atoms linked by chemical or metallic bonds. Typical time scales for phonons are $10 \mathrm{fs}-10 \mathrm{ps}$, depending on the size of the molecule and complexity of the atomic rearrangement required. This time-scale is considerably longer than for pure electronic excitations and provides a temporal window on coupled electronic-structural transformations.

Magnetic, ferroelectric, and multiferroic time scales for polarization reversal are the longest, typically $40 \mathrm{ps}$ to $10 \mathrm{~ns} .^{119}$ The long time-scale is due to the highly collective nature of magnetic and electric polarization in solids, often made up of large domains of atoms sharing a single polarization direction that interacts through long-range electric or magnetic fields with neighboring domains. Polarization reversal is a dramatic change in this large and complex collective structure that 
requires commensurately longer times to complete. In the case of ferroelectric reversal, atomic motion in addition to electronic reorganization is essential.

\section{Magnetic Moment reversal}

\section{Structural} Phonons

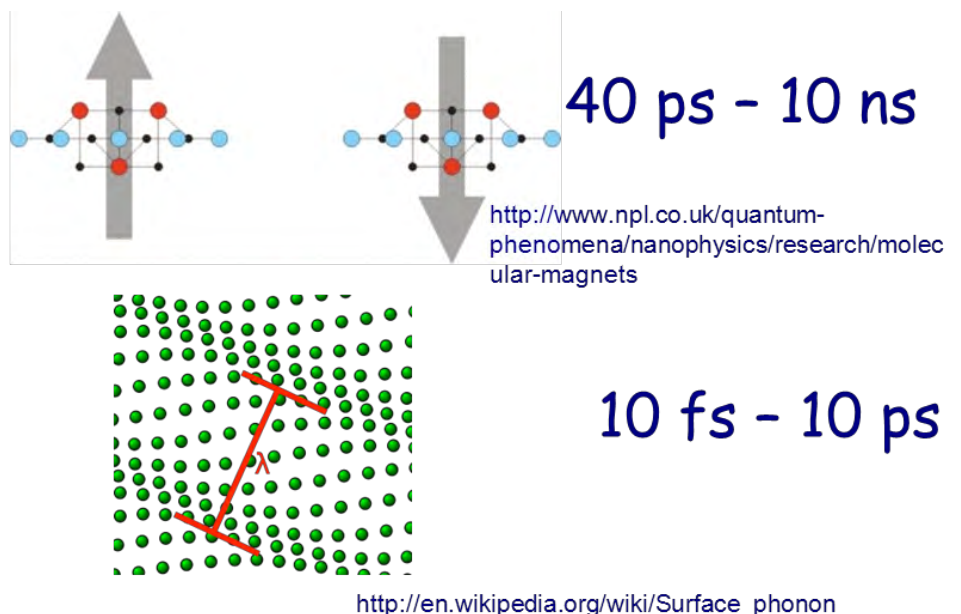

http://en.wikipedia.org/wiki/Surface phonon

\section{Electronic Energy levels}

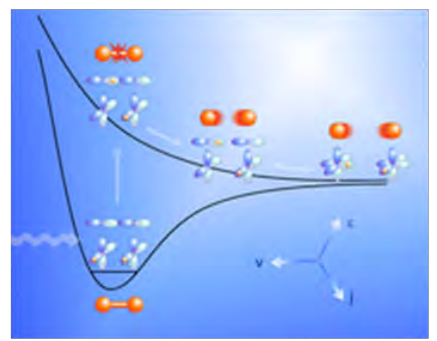

\section{0 as -100 as}

Phys. Chem. Chem. Phys., 2006, 8, 5581

Figure 21. Time-scale of dynamic processes in materials science (lower image ${ }^{120}$ ).

In this section, we explore the new scientific horizons that ultrafast electron microscopy opens for capturing the dynamics of materials at ps and faster time scales in three broad areas: interfaces, phase transitions and fluctuations, and radiation damage.

\subsubsection{Interface Dynamics}

\subsubsection{Solid-Liquid Interfaces}

Crystal growth phenomena raise a host of fundamental questions regarding liquid fluctuations and kinetic attachment rates at solid-liquid interfaces. Though amenable to simulation, the dynamics of atomic or molecular attachment at the solidliquid interface has not been observed experimentally. ${ }^{121} \mathrm{CdZnTe}(\mathrm{CZT})$ provides a timely example, important for the development of new radiation detector materials, both scintillators and semiconductors, with increased resolution, brightness, and ease of use. CZT semiconductor crystals are appealing room-temperature replacements for liquidnitrogen-cooled, high-purity Ge. However, growth of CZT is plagued by irreproducibility and defects thought to be a result of formation of Te-particles in the liquid adjacent to the solid-liquid growth interface. Recent modeling and simulation results illustrated in Figure 22 indicate that both compositional and structural fluctuations in the liquid phase may play a role in these growth defects. ${ }^{122}$ In addition, the crystallography of the melt interface can determine fluctuations and kinetic attachment rates. Atomically rough interfaces appear to be able to support faster growth rates, while interfaces that form facets can exhibit sluggish growth due to lateral step growth mechanisms. ${ }^{122-124}$ 

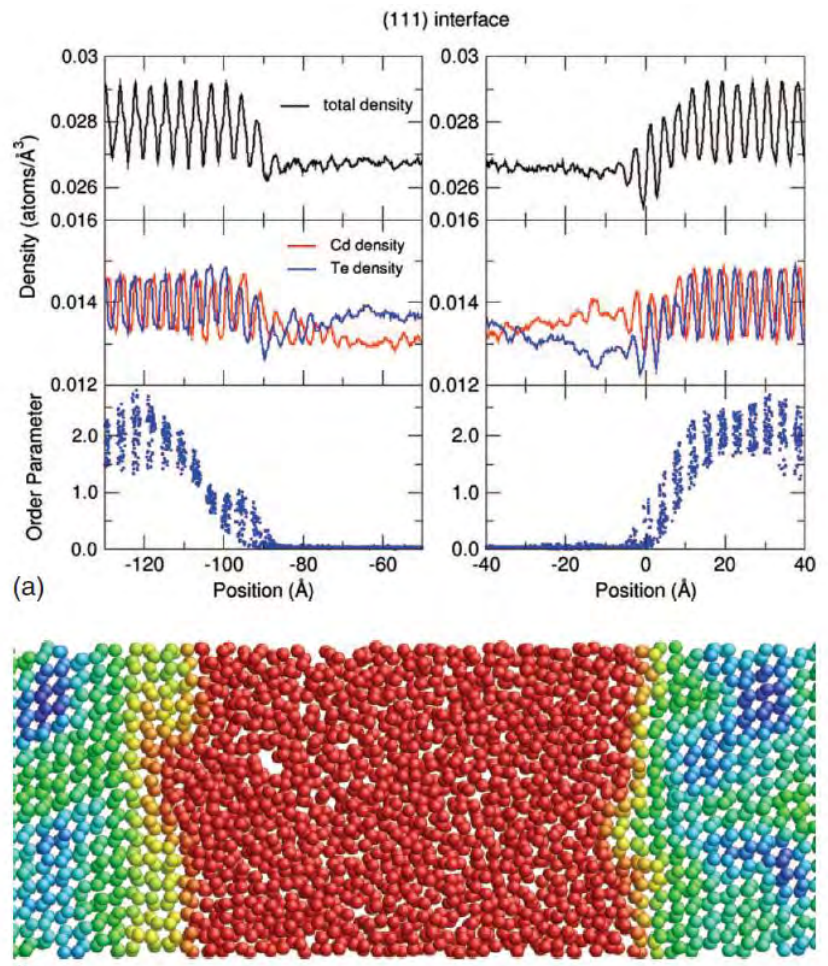

(b)

Figure 22. Simulation results for CdTe solid-liquid phase equilibria where the melt interface is normal to the (111) CdTe interface. Shown in (a) are fine-scale density plots of atomic composition and order parameter. Observe the CdTe fractionation in (a) and the interface faceting in (b) in the atomic coordination model. ${ }^{122}$

Provided that a reliable melting trigger can be found to initiate local melting in certain materials, ultrafast TEM has the potential to capture structural and compositional fluctuations at solid-liquid interfaces. A novel experiment would be to create a molten zone in a single crystal of CZT via laser-induced melting and to monitor the compositional and structural fluctuations within the molten zones as functions of crystalline anisotropy. For Si, the observed liquid structural units have lifetimes on the order of 10-20 ps, which puts this phenomenon in the right time-scale for UTEM analysis. In CZT, it can be anticipated that Te atoms will form chain-like structural units in the liquid. However, their lifetimes and interface adjacencies, which are of critical importance to the subsequent formation of Te-particles and other structural defects, are unknown. The formation of clusters within the liquid or the direct observations of certain growth defects at the solidliquid interface should be available during well-designed UTEM melting/solidification experiments. All of the structures and gradients observed in the molecular dynamics models shown in Figure 23 were obtained under quasi-equilibrium conditions over several ps. For the first time, UTEM may be able to capture such images directly, exploring the detailed validity of the models and inspiring the development of new physical approaches to interface behavior. 

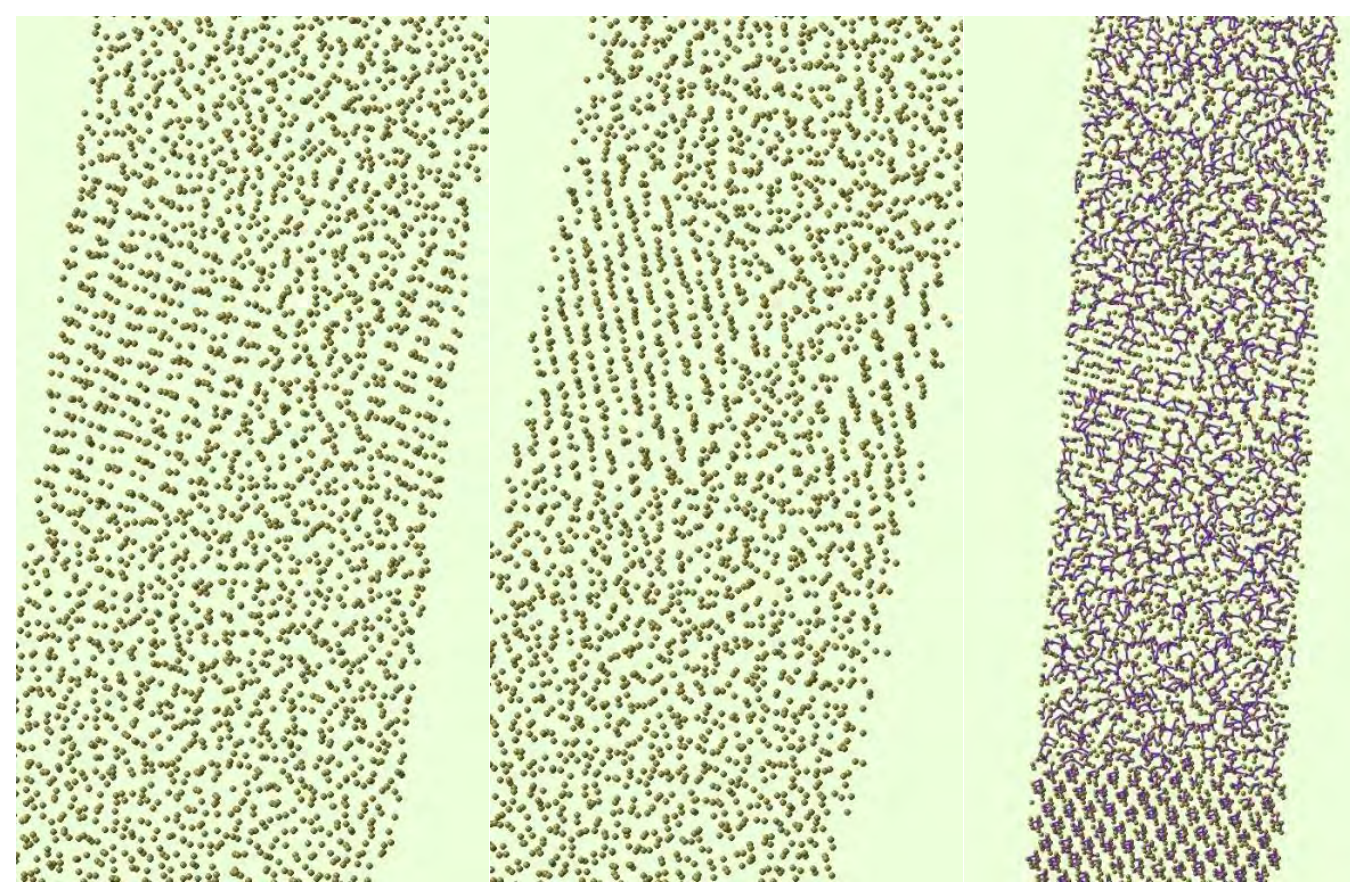

Figure 23. Atomistic models of liquid Te showing formation of solid nuclei in the liquid phase but not directly adjacent to the solid-liquid interface. Such clustering should be within the range of a well-designed UTEM melting experiment and would provide valuable details for CZT solidification that now can only be obtained via modeling and simulation. ${ }^{125}$

\subsubsection{Emergent Conductivity at Interfaces}

Recent discoveries reveal that the interface between dissimilar materials is rich with emergent behavior on a variety of length and time scales. ${ }^{124,126-129}$ On the long-distance scale, the atmosphere-ocean interface influences the rate of heat transfer from the ocean to atmosphere, and the rate of carbon dioxide diffusion from the atmosphere to the ocean. These processes are critically important to weather patterns and ocean acidification. On the small scale, the interface between $\mathrm{SiO}_{2}$, or more recently $\mathrm{HfO}_{2}$ and $\mathrm{Si}$, determines whether or not the billions of transistors in our personal computers turn on and off as designed. The interface between $\mathrm{LaAlO}_{3}$ and $\mathrm{SrTiO}_{3}$, two common semiconductors, results in a high-mobility, two-dimensional electron gas, which can also be magnetic or superconducting. UTEM capabilities focus on a range of length and time scales that, for the first time, can probe subtle relationships between atomic structure, composition, and quantum confinement on the one hand and a variety of long-range collective orders and industrially useful functionalities on the other.

Often, conductivity at interfaces of dissimilar semiconductors is different from that found in the bulk of either material. This difference occurs because interfaces affect energy levels for charged particles in pure materials in novel ways, for example, to bend downward or upward at the interface, thereby attracting and confining electrons or "holes" (missing electrons). Indeed, the operation of many advanced electronic devices, such as modulation-doped field-effect transistors, is based on this phenomenon. ${ }^{130}$ The detailed behavior of energy bands at semiconductor interfaces and the electrical conductivity is sensitively dependent on the system and its constituents: on the distribution of atoms at the interface, on electric fields and how they are screened, and on collective order that emerges. Moreover, interfaces of materials that are not completely immiscible often are inherently metastable. The thermodynamic tendency is for atoms to diffuse across the interface to reduce sharp gradients, leading to an intermixed region characterized by concentration gradients for the 
different atoms present. An energy barrier must be overcome to initiate the interdiffusion process. Yet, once this barrier is overcome, significant atom motion can and often does occur. Additionally, interdiffusion can be influenced by the presence of defects in the constituent materials. Understanding the atomistic dynamics of intermixing at crystalline interfaces requires probes such as those provided by UTEM.

In contrast to their usual insulating behavior, the interface between $\mathrm{LaAlO}_{3}$ and $\mathrm{SrTiO}_{3}$ can be conductive - even superconducting - at very low temperatures. ${ }^{126-128}$ It can be magnetic and even exhibit electron-hole bilayers with nanoscale separation. ${ }^{128}$ One possible explanation for the interface conductivity is a transfer of electrons from $\mathrm{LaAlO}_{3}$ to $\mathrm{SrTiO}_{3}$ to alleviate the so-called "polar catastrophe," which, in principle, results from placing a polar oxide $\left.(\mathrm{LaAlO})_{3}\right)$ in direct contact with a nonpolar oxide $\left(\mathrm{SrTiO}_{3}\right)$. This charge transfer process is predicted to result in the formation of a twodimensional electron gas (2DEG) at the interface that is only 1-2 nm deep and must occur if the atomic structure is ideal. Charge neutrality will dictate a conducting interface unless collective order, such as charge ordering or magnetism, arises. With current synthesis methods - pulsed laser deposition or the gentler molecular beam epitaxy-this interface may not be atomically abrupt. Any of the four metal ions- $\mathrm{La}^{3+}, \mathrm{Al}^{3+}, \mathrm{Sr}^{2+}$, and $\mathrm{Ti}^{4+}$-may interdiffuse and supply charge carriers in the interfacial region. If the conductivity is caused by chemical doping, the depth of the conductive plane will be influenced by the depth over which the electrically active dopant diffuses inward, ${ }^{131}$ as well as by local charge neutrality.

The dynamics of intermixing and the spatial confinement of the electric current near the interface can be determined with UTEM. Laser pulses can provide controlled heating to surmount the energy barrier to intermixing, while time-resolved imaging monitors the lattice intermixing and EELS spectral maps monitor the conduction electron penetration across the interface over areas sufficiently large to obtain a mesoscopic description of both processes. The process is shown schematically in Figure 24. Each heating pulse will provide some atoms with the energy required to overcome the activation barrier for diffusion. The lattice image and EELS spectral maps following each pulse will provide instantaneous information about the ensuing ionic motion. The energy per pulse will be increased in a systematic way to eventually drive the system from a non-thermal to thermal regime and to its melting point. By using substrates having different concentrations of point defects and step densities on the surface, UTEM will reveal how these defects affect the diffusion process.

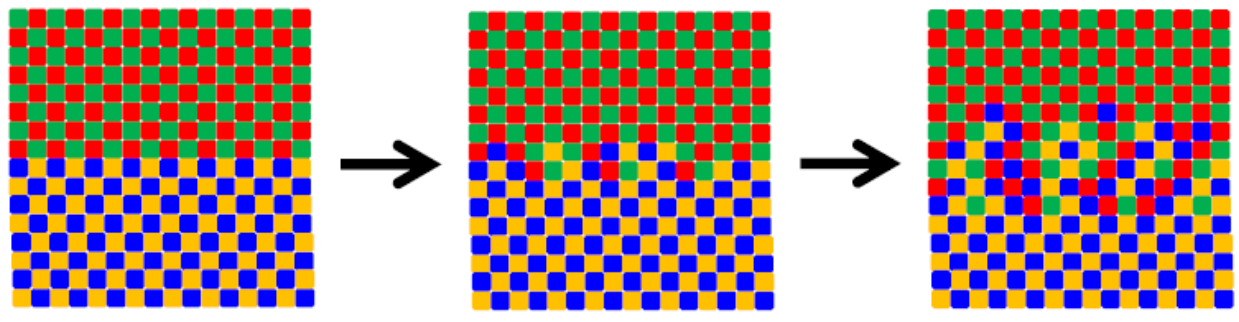

Figure 24. Schematic diagram of laser-pulse-induced interface mixing probed by UTEM.

UTEM experiments can be designed to image electrical current at the interface of insulators during in situ conductivity measurements. The actual current at the interface can be imaged in one of two ways. First, phase contrast in the lattice image will result from the presence of extra electrons in the conducting plane. Lattice images with microscope settings maximally sensitive to phase contrast will directly produce images of the current path near the interface. Second, for interfaces of complex oxides with transition metal (TM) cations at the $B$-site, the bottom of the conduction band is transition metal $\mathrm{d}$ character and, therefore, is temporally reducible by itinerant electrons in the current flow. Using the $\mathrm{LaAlO}_{3} / \mathrm{SrTiO}_{3}$ interface as an example, itinerant electrons in the conducting path in the $\mathrm{SrTiO}_{3}$ will reduce some fraction of the structural $\mathrm{Ti}^{4+}$ cations to $\mathrm{Ti}^{3+}$ over the time-scale of the EELS process. For example, if the current path includes one-tenth of the $\mathrm{Ti}$ atoms, then, at any one time, one tenth of the $\mathrm{Ti}$ ions will be $\mathrm{Ti}^{3+}$. The $\mathrm{Ti}_{2,3} \mathrm{EELS}_{\text {spectrum is }}$ 
sensitive to the charge state of $\mathrm{Ti}$. The distinct four-peak structure characteristic of $\mathrm{Ti}^{4+}$ (Figure 25) becomes a broad doublet when $\mathrm{Ti}$ is reduced to $\mathrm{Ti}^{3+}$. Thus, by obtaining $\mathrm{Ti}_{2,3}$ EELS maps as a function of time in stroboscopic imaging mode with sufficient time resolution, it will be possible to determine where the conduction electrons are relative to the interface. In turn, this procedure will allow the thickness of the conducting plane to be directly measured, shedding light on the mechanism of conductivity. If the depth of the conducting plane matches that of interdiffused La, then La doping of the STO is the most likely explanation for conductivity.

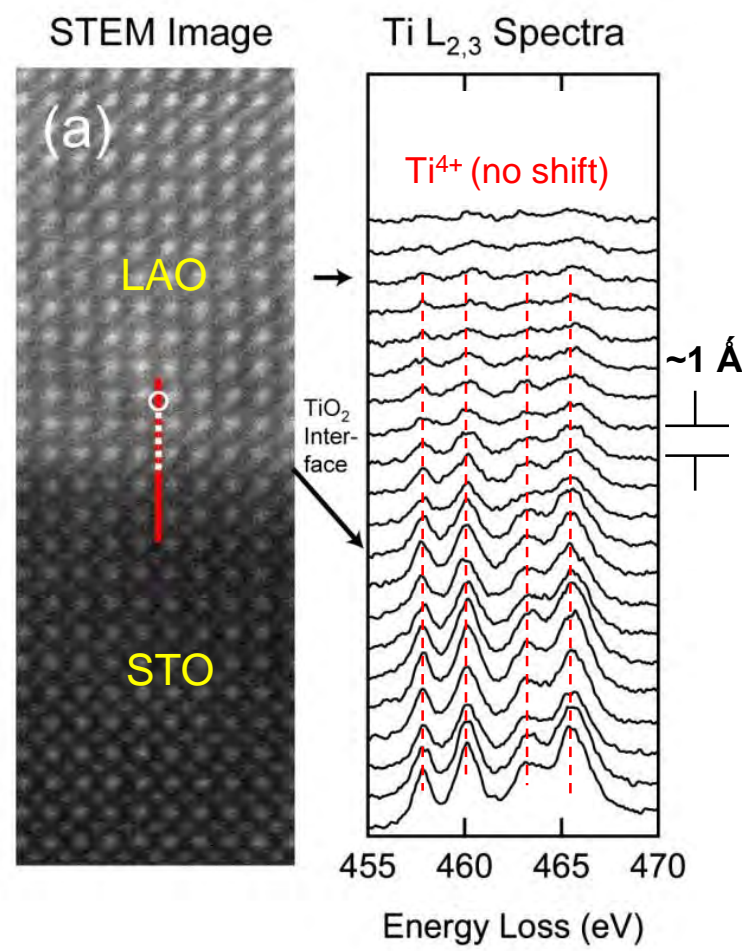

Figure 25. STEM image (left) and $\mathrm{Ti} \mathrm{L}_{2,3}$ EELS spectra obtained along the red line normal to the interface (right) for $\mathrm{LaAlO}_{3} / \mathrm{SrTiO}_{3}$ interface. The persistence of the Ti EELS peaks well into the $\mathrm{LaAlO}_{3}$ is due to $\mathrm{Ti}$ outdiffusion. No $\mathrm{Ti}^{3+}$ is seen along this line. ${ }^{131}$

UTEM has the potential to explore these emergent interface phenomena with unprecedented clarity. Picosecond resolution is required to track the irreversible diffusion of atoms across the interface in response to heat pulses. The dynamics of intermixing can only be monitored by recording the change in atomic positions following each series of laser heat pulses. The energy per pulse can be incrementally increased after a certain number of pulses at some constant energy in order to drive the process in a controlled way. Likewise, determining the time evolution of the itinerant electron distribution can only be done by collecting a series of time-resolved lattice and EELS images under the influence of an applied electric field. There is no need for laser heating pulses in this experiment. However, time-resolved lattice images obtained in the stroboscopic mode are essential.

\subsubsection{In situ Crack Propagation}

The initiation and propagation of cracks affects all real-world materials applications and results from a host of causes depending on the material system. Cracking can occur quickly by exceeding the basic strength limits of the material or slowly from environmental degradation by radiation or corrosive liquids or gases. There are a multitude of environmental 
conditions that accelerate cracking depending on the material type. Polymers are often weakened due to exposure to radiation that slowly causes chain scission, thereby weakening the entire structure, whereas metals and ceramics often suffer from corrosion as a result of exposure to liquid and gaseous environments. Regardless of why cracking occurs, it typically can be broken down into three stages: 1) initiation, 2) propagation, and 3) catastrophic failure. Catastrophic failure is the end result of sufficient initiation and propagation of cracks. Hence, it is the initiation and propagation, as well as the environmental factors that could affect these phenomena, which are most important to understanding the underpinning mechanisms of cracking.

Understanding cracking as a failure mechanism using electron microscopy was long confined to ex situ post-cracking examinations. Cross-sections of the cracks would be thinned to electron transparency, and static snapshots of the microstructure then could be analyzed and related to the propagation mechanism. The region immediately surrounding the crack tip provided information as to what was occurring structurally within the material to drive the crack's propagation. However, this ex situ cracking evaluation did not reveal the crucial structural information necessary to truly understand how the material surrounding the crack deformed during propagation. With the advent of in situ holders, the ability to observe the propagation of cracking became possible. Robertson et al. ${ }^{132}$ examined dynamic interactions between matrix dislocations and grain boundaries near propagating cracks in thinned TEM specimens while the sample was being strained at increments of $4 \mathrm{~nm}$ per second and recorded at 1/30th of a second intervals (Figure 26). This work, performed on brittle and ductile materials ranging from $\mathrm{Ni}-\mathrm{S}$ and $\mathrm{Ni}_{3} \mathrm{Al}$ that failed intergranularly to $\mathrm{Ni}$ and $\mathrm{Ti}$ that failed transgranularly, demonstrated that in all materials, dislocations were fully incorporated into grain boundaries as they approached them. The grain boundaries would accommodate a number of dislocations before responding by either dislocation emission or fracture. Brittle materials would respond by nucleation of intergranular cracks, while, in ductile materials, grain boundaries would act as dislocations sources. Additional work illustrated how the introduction of $\mathrm{H}$ gas could affect the stress-relief mechanism.

More recent in situ straining research has been performed on a range of materials, from ceramics ${ }^{133}$ to nanocrystalline gold thin films ${ }^{134}$ using similar straining holders. The issue with these holders is that the strain applied across the disk cannot be directly quantified. Haque and Saif ${ }^{135}$ demonstrated they could use MEMS to test 100 -nm-thick, freestanding aluminum films, which could be strained under more controlled loading. In this study, the Al film (100-nm thick) consisted of 50-nm grains, and they observed no dislocations ahead of the propagating crack tip (Figure 27).

This body of research lays the groundwork for performing in situ crack initiation and propagation using ultrafast electron microscopy experiments. A variety of approaches, from using full-sized ( $3 \mathrm{~mm})$ TEM disks to much smaller and more discrete MEMS straining devices, provides a valuable source of research suggesting this type of experiment is possible. The initial experiments by Robertson et al. ${ }^{132}$ were performed on the timeframe of $1 / 30$ th of a second, leaving several orders of magnitude to probe the creation and movement of dislocations near propagating crack tips. In moving from these in situ experiments to the ultrafast regime, the main issue is controlling the applied strain using an ultrafast laser pulse. One approach is to use a MEMS device with a tensile setup that expands when pulsed with a laser. The crack could first be initiated in the sample, and then with known laser pulses and thermal expansion of the MEMS device, small, controlled tensile forces could be used to propagate the crack further. A similar holder could be envisioned to induce gaseous or liquid environments to test the response of crack propagation. Studies of deformation and dislocation formation near the propagating crack on the tens of picoseconds time-scale would provide invaluable information on the mechanism of crack propagation and effective mitigation strategies. 


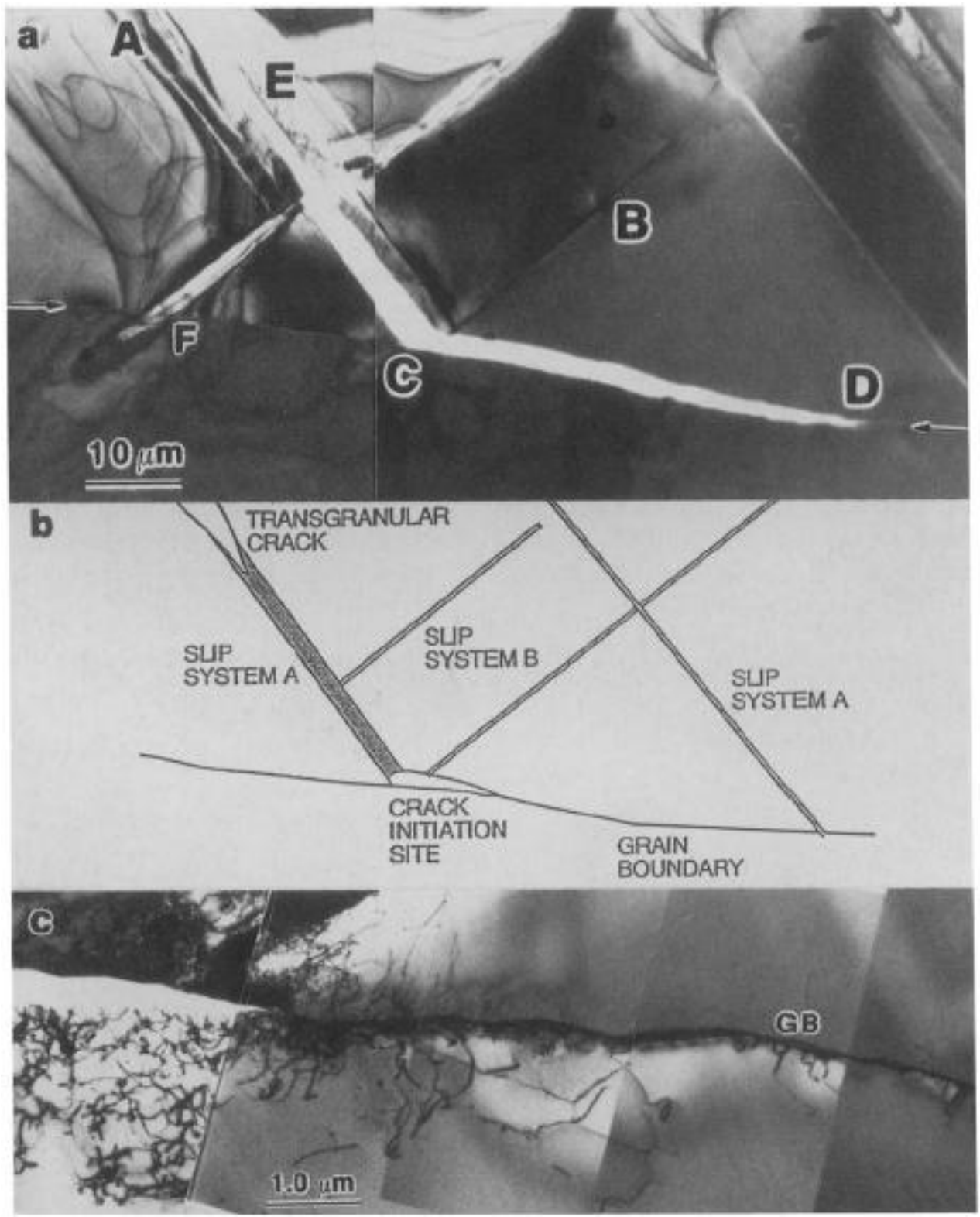

Figure 26. Nucleation and propagation of an intergranular crack. ${ }^{132}$

\subsubsection{Polarization Dynamics in Ferroelectric and Multiferroic Materials Systems}

Ferroelectric and ferromagnetic materials share the common feature of a spontaneous polarization that can be switched between two stable states, enabling non-volatile information storage. However, the underlying origins of ferroelectric and ferromagnetic polarization and their switching mechanism are qualitatively different. Magnetic moments arise from the angular momentum of electrons moving in closed atomic or molecular orbits or from the electron's intrinsic spin and always appear as dipoles or higher-order moments. In contrast, electric moments arise statically from asymmetric positive ionic and negative electronic charge distributions composed of clearly distinct sets of monopole charges. Electric moments are intimately linked to the positions of positive ions, and polarization reversal requires displacement of ions 
within the unit cell. Because electrostatic energies typically are much larger than magnetostatic energies, the activation barrier for electric polarization reversal typically is much larger than for magnetic reversal.

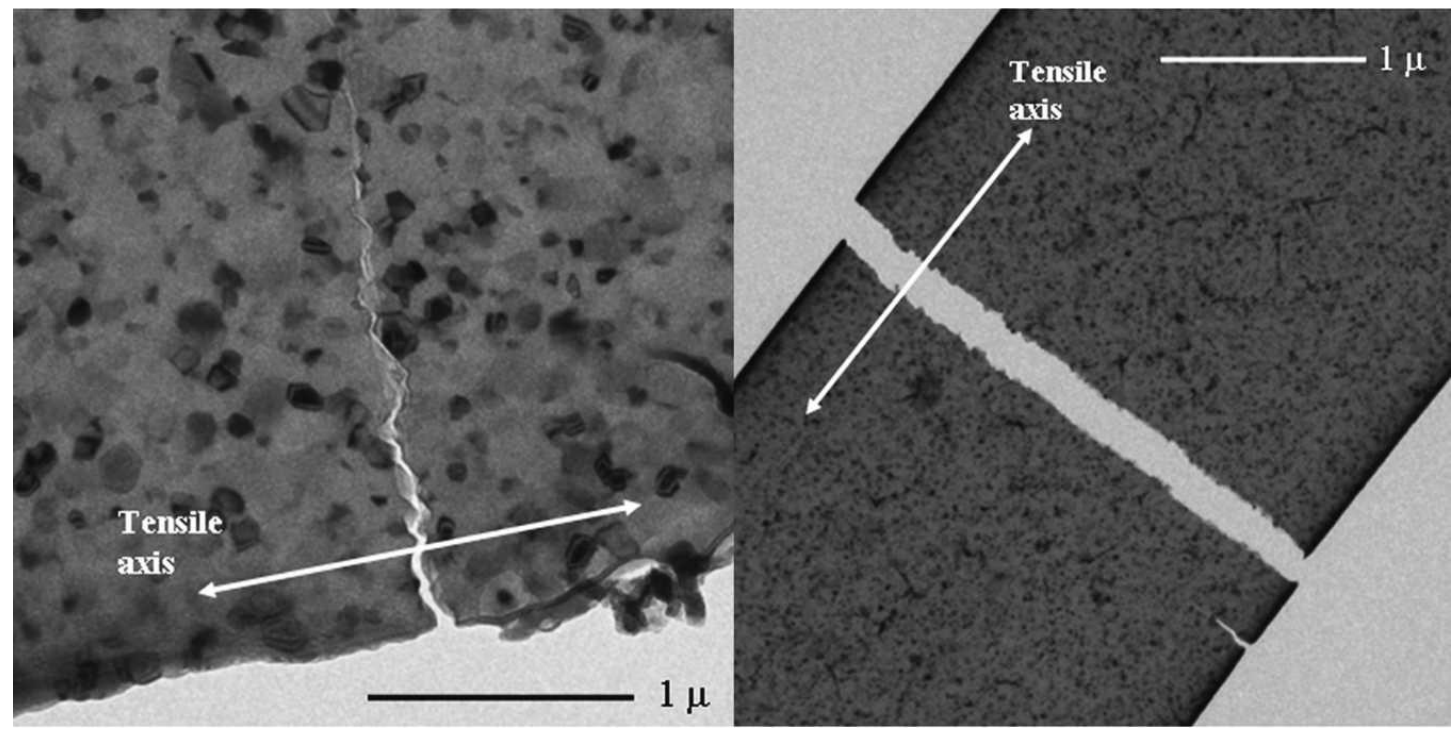

Figure 27. In situ testing of 100-nm-thick Al film using a MEMS device. ${ }^{135}$

The switching time and minimum volume for a stable polarization in electric and magnetic materials drives memory applications. Smaller memory bits can be cheaper and faster, enabling new horizons for portable memory storage. The long history of magnetic memories has produced a wealth of information about their domain structures, polarization stability, reversal mechanisms and switching times, although new nanoscale magnetic structures reveal polarization morphologies and switching dynamics that are qualitatively different from their larger counterparts. In contrast, much less is known about the dynamics of ferroelectric materials. The intimate coupling of electric polarization reversal to atomic displacement suggests that intrinsic polarization switching times are of order of phonon mediated processes-in the ps regime - an expectation recently supported by terahertz spectroscopy. ${ }^{136}$ UTEM will be capable of imaging electric polarization, domain wall configurations, and atomic structure with 10 ps resolution allowing direct observations of ferroelectric dynamic behavior.

There are several fundamental challenges for ultrafast electron microscopy in ferroelectric and multiferroic materials, including:

1. Ferroelectric switching dynamics of the polarization reversal and atomic displacements of positive and negative ions, such as $\mathrm{Pb}^{2+}$ in $\mathrm{Pb}\left(\mathrm{Zr}_{\mathrm{x}} \mathrm{Ti}_{1-\mathrm{x}}\right) \mathrm{O}_{3}(\mathrm{PZT})$ or $\mathrm{Bi}^{2+}$ in $\mathrm{BiSr}_{2} \mathrm{Ta}_{2} \mathrm{O}_{9}(\mathrm{SBT})$ and $\mathrm{O}^{2-}$ in perovskite structures. This requires spatial resolution at sub-nm levels and temporal resolution in the $\mathrm{ps}-\mathrm{ns}$ range. Fundamental questions include systematic studies of confinement phenomena as a function of size from nanoscale to macroscale in lithographically patterned thin films and in three-dimensional nanoparticles. Fundamental research directions include:

- Ion displacement in the bulk and at electrode/ferroelectric layer interfaces and their effect on polarization switching

- The effect of point defects, such as oxygen vacancies and interfacial and bulk structural defects on polarization switching dynamics, including polarization fatigue, imprint, and retention phenomena. 
2. Studies of ferroelectric switching dynamics at the polarization domain level looking at domain wall motion during polarization switching in ferroelectric thin films. These studies would be performed on ferroelectric nanostructures to investigate the effect of nanoscale confinement on domain wall dynamics.

3. Studies of ferroelectric/magnetic polarization interaction in multiferroic materials to investigate the dynamic correlation of ferroelectric polarization reversal due to ion displacement in the lattice and magnetic vector switching dynamics and vice versa.

The fundamental studies of nanoscale phenomena in ferroelectric and multiferroic materials performed with UTEM will provide the knowledge required to design the next generation of high-density, non-volatile memories that may revolutionize the multibillion dollar non-volatile memory market.

\subsubsection{Resistive Switching Memory Materials}

\subsubsection{Conducting Filament Switching}

Resistive switching is a new paradigm for non-volatile memories that writes stable conducting or insulating states in real time for storing information. As illustrated in Figure 28, the conducting state is "SET" by an applied voltage, and the insulating state is "RESET" by an opposite voltage. The states can be read by smaller applied voltages. Such memories demonstrate promising behavior with switching times in the range of $5 \mathrm{~ns}$, resistivity ratios between the conducting and insulating states on the order of $10^{7}$, and retention times approaching one year. ${ }^{137}$ The configuration of resistive memories is compatible with three-dimensional stacking, allowing a qualitatively new horizon for achieving high density.

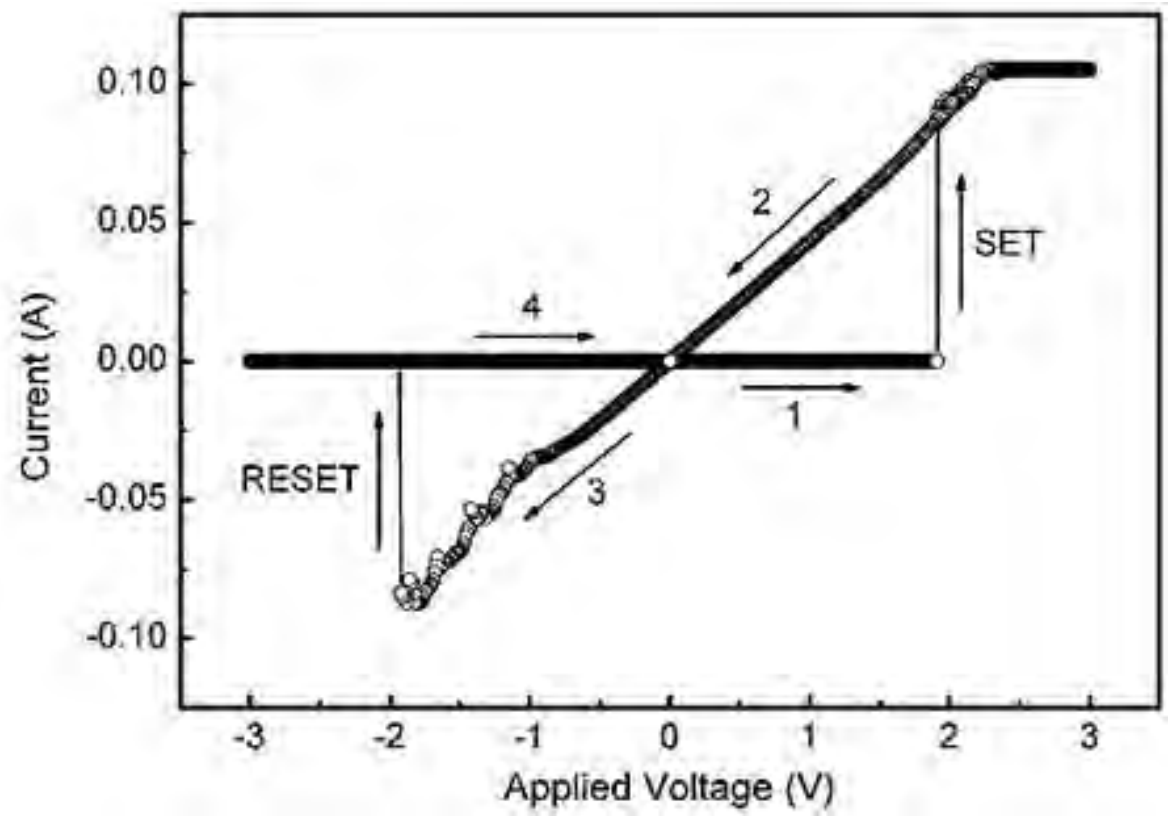

Figure 28. Switching behavior of resistive memory based on $\mathrm{ZnO}$ doped with $\mathrm{Mn} .{ }^{137}$

The switching mechanism for these materials is not understood, but it is thought to arise from the electrochemical oxidation, migration, and growth of conducting filaments across the insulating layer driven by the applied voltage (illustrated in Figure 29). The ns switching time reflects the completion of the entire process, including oxidation, migration, and filament growth. Each step will take shorter times on the order of hundreds of ps. Ultrafast electron 
microscopy can probe the individual dynamic steps of the switching process with the spatial and temporal resolution needed to capture them - imaging for the migration of ions and growth of filaments and EELS for identification of the filament's chemical composition. This information is critical for understanding the basic phenomenon of resistive switching and for creating the next generation of fast, high-density, non-volatile memory devices.
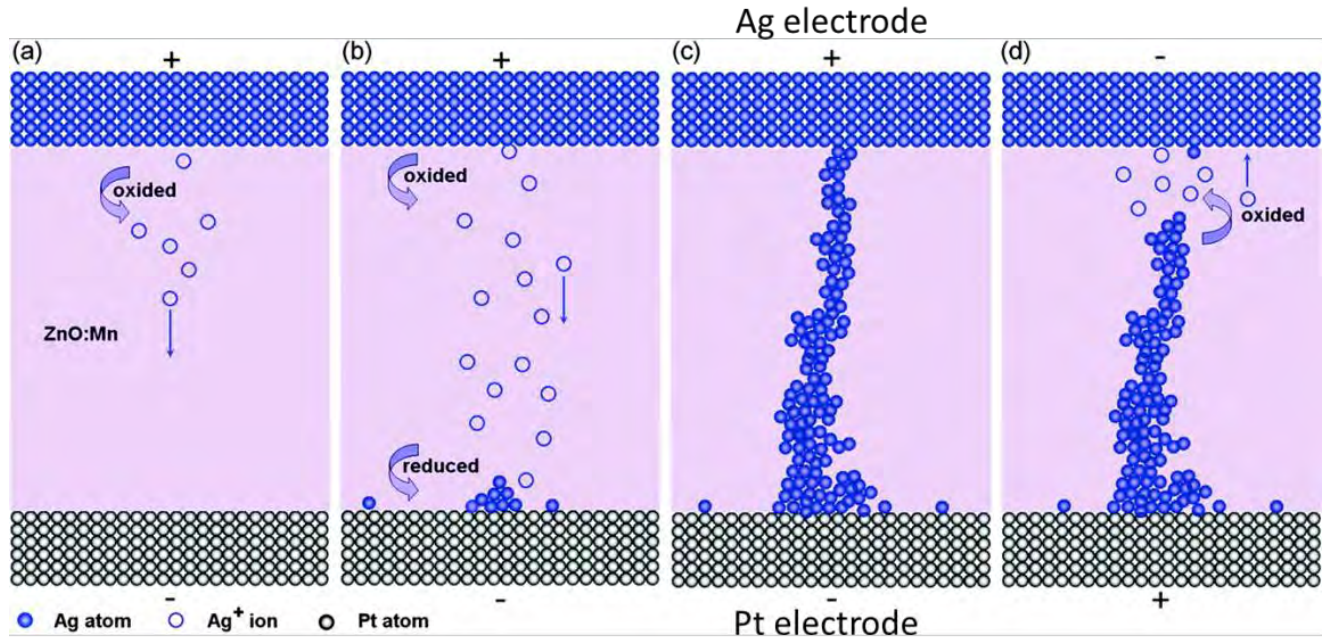

Figure 29. Cartoon of the electrochemically driven growth of a conducting silver filament within an insulating $\mathrm{ZnO}: \mathrm{Mn}$ layer of a resistive memory cell. Ag atoms from the top electrode are ionized and electromigrate to the bottom electrode, where they are reduced to neutral Ag and accumulate, forming a conducting filament spanning the layer. ${ }^{137}$

\subsubsection{Correlated Electron Switching}

A second and more recent mechanism for resistive switching relies on a bulk transition of a conducting layer to an insulating state driven by strong correlations among conducting electrons, giving rise to a Mott insulator. Switching from the Mott insulator to the conductor is thought to be driven by tunneling of excess electrons into the Mott state under the influence of a sufficiently strong electric field; these excess electrons screen the Coulomb interaction energy among localized electrons and lower the correlation-induced Mott bandgap to zero, producing a metal. ${ }^{138}$

Correlated electron switching differs fundamentally from the filament formation switching already described above - for correlated electron switching no atomic migration or reconfiguration is required, as the transition takes place entirely within the electronic system.

One example of Mott transition switching is in $\mathrm{NiO}$, where the critical concentration of electrons just at the Mott transition value is achieved by "fine-tuning" via carbonyl (CO) ligand doping at the interface with one of the electrodes. ${ }^{138}$ The carbonyl ligands regulate the number of oxygen vacancies and the surface excess of metal ions and electrons. Finetuning the electron concentration with carbonyl doping puts it just at the threshold of the Mott transition, allowing tunneling of a few excess electrons driven by a modest applied voltage to destabilize the Mott state and create the metallic state.

The Mott transition approach to resistive switching offers the advantage of an all-electronic transition without the need for slow atomic motion to build and destroy a conducting filament across an insulating layer. It also is rather complex, depending on fine-tuning of the charge concentration by controlling the concentration of carbonyl ligands and oxygen 
vacancies at the electrode interface. Mapping the statics and dynamics of these electronic processes by imaging the spatial structure and probing the composition and valence dynamics with EELS using UTEM is critical to understanding the mechanism of Mott transition switching in resistive memory. Mott transition switching may enable a new generation of high-density Correlated electron resistive Random Access Memory (CeRAM) that could replace FLASH memory that now dominates the non-volatile memory market.

\subsubsection{Phase Transitions and Fluctuations}

\subsubsection{Solid-State Phase Transformations}

A key scientific feature of dynamic in situ experiments is their unique ability to probe the time evolution of condensed matter systems "on the fly" as they occur. Consequently, fundamental atomic- and electronic-scale mechanisms can be examined for a broad range of condensed matter phenomena, including structural changes and electronic transitions occurring during solid-solid phase transformations, melting and resolidification, and nucleation and growth.

Solid-solid phase transformations are a fundamental and pervasive phenomenon of materials science whose mechanisms, kinetics, and microstructural development hold many mysteries accessible with time-resolved electron microscopy. The study of phase transformations often yields high impacts. The industrial revolution of the late 18th and 19th centuries was enabled by manipulation of steel microstructures through heat treatments that triggered massive and martensitic transformations, an early success of metallurgy. ${ }^{139}$ Phase transformations are equally important today, not only in steels that now include stainless, ${ }^{140}$ high-strength, ${ }^{141}$ and dual-phase steels ${ }^{142}$ but also in electronic materials, such as phase change materials for non-volatile memory ${ }^{143}$, medical applications, such as shape memory materials for prosthetic implants ${ }^{144}$ and biological processes ${ }^{145}$. The kinetics of phase transformations are the key feature determining the response of materials to dynamic and impact mechanical loading. ${ }^{146}$

Two dynamic processes control phase transformations and the resultant microstructures that govern properties: the nucleation of new phases, and their growth within the parent phase. These two processes are not independent. Their interaction can either suppress or stimulate the process of nucleation. Suppression of nucleation occurs when growth of the new phase consumes potential nucleation sites embedded in the parent phase. Stimulation occurs when the stress at the moving phase boundary creates new defects in the parent phase that become nucleation sites, usually associated with martensitic phase transformations. This stimulation of nucleation often is referred to as the "autocatalytic effect" and is responsible for the creation of distinct grain morphologies and grain refinement that provide strength in these materials.

\subsubsection{Nucleation}

The dynamics of nucleation are commonly viewed in the framework of classical nucleation theory as a competition among three contributions to the total energy: volume, interfacial, and strain energies (after Porter and Easterling ${ }^{147}$ ). While this framework is generally applicable, the number of quantitative investigations of nucleation is limited because nuclei sizes are small and the event is typically rapid — conditions perfectly suited to study by time resolved electron microscopy.

Recrystallization ${ }^{148}$ and martensite transformations ${ }^{149}$ are two specific examples where the theory falls short. In these cases failure is thought to arise from the role of pre-existing defects in promoting nucleation. In the case of martensitic transformations, ${ }^{150}$ it has long been known that nucleation is a heterogeneous process. ${ }^{151}$ It is thought that lattice defects, such as dislocations, twin boundaries, and grain boundaries, have atomic structures that are favorable to the formation of a 
martensite phase. ${ }^{152}$ However, nucleation sites for martensite have never been directly observed. Rather, they are inferred from microstructure morphology and interface structure observed in static post-transition measurements. ${ }^{153}$ In most descriptions of martensite transformation, nucleation is considered to be barrierless and governed by interface mobility. Thus, the defect structures at the interface and their interactions with local microstructure dominate the macroscopic transformation behavior, where rates are controlled by the potency of initial sites and those generated by autocatalytic nucleation. UTEM investigations of the interface dynamics and the nucleation of new martensite ahead of the propagating phase front will open new horizons to observe, understand, and control the detailed mechanisms of phase transformations and to better predict the transformation behavior and final microstructures.

The description of intermediate metastable phases that connect the structure of the parent and new phases is another failure of classical nucleation theory. This is a rich, controversial, and, potentially, widespread phenomenon that remains largely unexplored experimentally. Nucleation of intermediate metastable phases often is suspected when an unexpectedly low energy for nucleation is observed experimentally. The classic example is the formation of Guinier-Preston zones in alloy systems. ${ }^{154}$ Other examples of unexpectedly low activation energy for nucleation are steels undergoing the austeniteto-ferrite transformation ${ }^{155}$ and strongly driven systems with large undercoolings. ${ }^{156}$ These recent findings have generated interest and controversy ${ }^{157,158}$ concerning the prevalence and nature of intermediate metastable nucleation phases. There are no actual observations of nuclei with metastable structures outside the specialized case of Guinier-Preston zones, and observations of nucleation events of any kind have been rare. ${ }^{159}$ UTEM is required to explore the phenomenon of intermediate metastable nucleation sites that promote the phase transition and connect the crystal structure of the parent and new phases. These fleeting phenomena are invisible to static probes applied before and after the phase transition.

Even where classical nucleation theory is thought to be a sound framework ${ }^{160}$, details of the nucleation event have a significant impact on the kinetics, degree of completion of the phase transition, and the perfection of the new phase. For example, one of the major shortcomings of theory is its inability to accurately predict interfacial energies, a key element of the classical nucleation picture. Detailed observations of the interfacial structures combined with electronic structure calculations using DFT may be used to predict interfacial energies and offer better prediction of transformation behavior.

\subsubsection{Growth}

The structure and kinetics of a moving martensitic interface hold many unexplored opportunities for ultrafast electron microscopy. Static snapshots of the structures of these interfaces have been characterized ${ }^{161}$ and modeled traditionally with the Phenomenological Theory of Martensite Formation ${ }^{162,163}$ and, more recently, by the Topological Theory of Martensite Formation, ${ }^{164}$ which models the interface as a network of defects that more directly relates the crystallography of the interface to the kinetics of the transformation. These models have shown great success in modeling the static interface. However, due to the difficulty of observing such a rapidly moving structure, the structure of the moving interface and its role on subsequent nucleation events are still a mystery. The situation is analogous to the structures of static versus a moving dislocation, ${ }^{165}$ which are predicted to have significantly different core structures.

Characterizing the interface as an array of defects implies certain behaviors. For example, there should be situations in which defect interactions kinetically promote the growth of the martensite and increase the interface mobility, while others impede the interface. These growth dynamics determine martensite grain morphology and the resulting mechanical properties. Another example is the interaction of interface dynamics with the nucleation process. As an interface propagates, it sweeps up potential nucleation sites, but the stress fields from the defects also should promote nucleation in the parent phase ahead of the front. Ultrafast electron microscopy observations can offer insight into these issues and link crystallography and kinetics to better predict and engineer martensite microstructures. 
In summary, time-resolved electron microscopy provides unprecedented opportunities to capture directly on the fly and elucidate changes in the atomic and electronic configurations occurring during phase transformations in materials and condensed matter systems. Consequently, in situ, time-resolved measurements at microscopic length scales likely will form the basis of revolutionary scientific advances for achieving a true fundamental understanding of phase transformations in condensed matter. An important overarching goal of time-resolved electron microscopy is to perform experiments on the time and length scales of numerical simulations. Increasing computational power enables simulations of longer real times and larger physical systems. While the ever-increasing reach of high-performance computational power continues to extend the boundaries of length scales available for numerical simulations, extending the time-scale of such simulations is problematic and remains an active area of research. Experimental, time-resolved UTEM investigations at the atomic length scale play a singularly important role in filling this knowledge gap.

\subsubsection{Fluctuations in Correlated Electron Materials: a "Killer App" for Ultrafast Electron Microscopy}

Fluctuations are fundamental to every field of physical and human behavior, from materials to meteorology, cosmology, and politics. In materials science, fluctuations accompany and promote phase transitions and, sometimes, leave a telltale fingerprint of the transition over large areas of phase space. The connection between fluctuations and phase transitions usually is characterized statistically through thermodynamic scaling laws that average over time and space. However, the dynamic behavior of fluctuations - the local evolution of their spatial, temporal, and energetic extent - remains a mystery. UTEM offers new spectroscopic and imaging tools that can look beyond scaling and begin to remove the obscurity clouding the fundamental dynamics of fluctuations. The qualitative differences between thermal and quantum fluctuations, for example, could be explored in both time and space domains.

In strongly correlated electron materials, ${ }^{166,167}$ the electron-electron correlation energies, kinetic energies, and ordering energies are of comparable size, producing a delicate balance of competing states, including: superconductivity, magnetic and orbital order, lattice distortions, and delocalized versus localized carriers. The small energy separation of these competing states produces large fluctuations, a dominant feature of strongly correlated electron systems. This delicate interplay of competing states produces a rich variety of emergent phenomena, such as the unique metal-to-nonmagnetic insulator transition in $\mathrm{VO}_{2}(340 \mathrm{~K})$, the charge ordering transition in rare earth nickelates $\mathrm{RNiO}_{3}(400-600 \mathrm{~K})$, and the high-temperature superconducting transition in the cuprates $(40-160 \mathrm{~K})$, as well as a high sensitivity to external stimuli including magnetic fields, electric currents, light pulses, and quenched disorder including sample defects and strains. A dramatic example of such sensitivity is the appearance of transient superconductivity arising in stripe-ordered $\mathrm{La}_{1.675} \mathrm{Eu}_{0.2} \mathrm{Sr}_{0.125} \mathrm{CuO}_{4}$ in response to ultra-short laser pulses. ${ }^{168}$

Strong electronic correlations cannot be approached theoretically as a perturbation in the independent electron picture, but must be taken into account in the initial formulation of the electronic behavior of the problem, a demanding and still elusive objective. Nevertheless, in the last decade, great advances have been made in simulating the dynamics of strong electronic correlations. Experimental and theoretical studies of strong correlation have broad support within the science and applications communities, as well as the agencies funding them. Often, the vision of understanding emergence in strongly correlated electron systems at the microscopic, quantitative, and predictive levels is included in lists of scientific Grand Challenges. ${ }^{166}$

A hallmark of strongly correlated materials is quasi-static and dynamic nanoscale electronic inhomogeneity that arises due to the competing and almost degenerate ordered states, as well as the sensitivity to local perturbations such as charged impurities. Such electronic inhomogeneity has been directly imaged for example in BSCCO and the recently discovered FeAs-based superconductors using STM ${ }^{169,170}$ (Figure 30). These static images of electronic inhomogeneity introduce the 
question of what analogous dynamic behavior might be in play, a question that can be investigated with ultrafast electron microscopy.
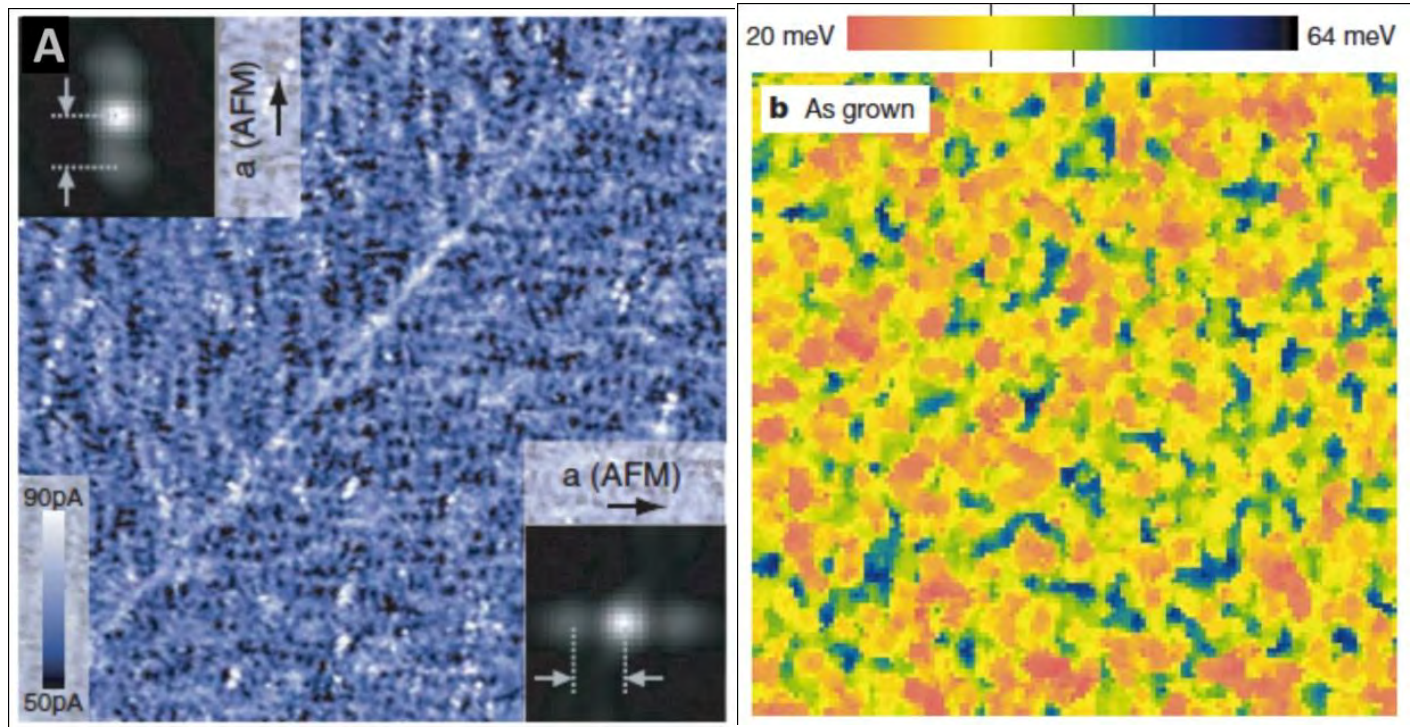

Figure 30. Illustrations of nanoscale inhomogeneity arising in correlated electron systems. A) STM current image of antiferromagnetic $\mathrm{Ca}\left(\mathrm{Fe}_{1-\mathrm{x}} \mathrm{Co}_{\mathrm{x}}\right)_{2} \mathrm{As}_{2}$ acquired at $4.3 \mathrm{~K}^{169}$. The diagonal line is an orthorhombic twin boundary. The insets show fast Fourier transforms of the image contrast. The frame size is $96 \mathrm{~nm} \times 96 \mathrm{~nm}$. B) Distribution of superconducting gap values in as-grown BSCCO at $4.2 \mathrm{~K} .{ }^{170}$ The image size is $56 \mathrm{~nm} \times 56 \mathrm{~nm}$.

Conventional phase transitions are governed within the critical region by thermal fluctuations where the relevant behavior becomes classical as thermal fluctuations dominate quantum fluctuations. In contrast, at quantum critical points, the condition $\mathrm{T}=0$ eliminates thermal fluctuations from the picture and brings quantum fluctuations to the forefront as the promoter of the transition. Even for conventional phase transitions, quantum fluctuations still occur and remain influential in the behavior around the transition region. While thermal fluctuations slow as they become critical at the transition, the quantum fluctuations follow only the dynamics determined by the energy differences and other distinctions between the competing phases. Imaging via ultrafast electron microscopy can enable the separation of thermal fluctuations from quantum fluctuations and direct observation of their effect in promoting or inhibiting phase transitions, which recently was predicted for glass formation. ${ }^{171}$

\subsubsection{Strongly Correlated Electrons and UTEM}

UTEM is uniquely poised to advance the understanding of strongly correlated electron systems. The exquisite spatial, temporal, and energy resolution that can be achieved will enable direct imaging of the static and dynamic evolution of the correlated states.

While ordered states that are coupled to lattice degrees of freedom (such as orbital and stripe order) can be directly imaged, the detection of superconducting or magnetic order that occurs primarily in the electronic system requires spectroscopic information. High-resolution EELS is indispensible for obtaining a complete picture of the correlated electron states. Energy resolution of $\sim 10 \mathrm{meV}$ in UTEM may be achievable either in stroboscopic operation mode ${ }^{172}$ or through pulse shaping of the electron bunch. This would be sufficient to image the superconducting gap in a wide range of 
high- $\mathrm{T}_{\mathrm{c}}$ superconductors and the spin-wave gaps and charge transfer gaps in Mott systems. By exposing the sample to laser pulses of various shapes, coherent phonon beams can be created in the material. When tuned to the resonant frequencies of certain bonds, such pulses have been shown to induce non-thermal melting of orbital order, for example, in $\mathrm{La}_{0.5} \mathrm{Sr}_{1.5} \mathrm{MnO}_{4 \cdot}{ }^{173}$ Using ultrafast electron microscopy, entirely new investigations of non-thermal melting dynamics in correlated electronic systems appear feasible.

With thermal fluctuations governed by $\Delta \mathrm{T}=|\mathrm{T}-\mathrm{Tc}|$ and with temperature stability of $\Delta \mathrm{T} \sim 0.2 \mathrm{~K}$ (for temperatures near $\sim 300 \mathrm{~K}$ ), the thermal energy scale of $0.01 \mathrm{meV}$ translates to a time-scale of $\sim 100 \mathrm{ps}$ - well within the range of ultrafast electron microscopy. UTEM will reveal the unique dynamic signature of the fluctuation behavior near phase transitions. Fluctuations are manifested as "patches" of one phase surrounded by another with the length scale of a patch equal to the correlation length and the lifetime of the patch equal to the inverse fluctuation frequency. Because the coherence length diverges and the fluctuation frequency slows to zero at the transition temperature, the space and time scales of the fluctuation dynamics are guaranteed to come within range of UTEM. Snapshots of fluctuations or, in stroboscopic mode, movies of their time evolution will show a dynamic patchwork or checkerboard of two phases, where the size of the patches reflects the correlation length and the dwell time of the patches reflects the fluctuation frequency. At first order transitions, such as in $\mathrm{VO}_{2}$, the nucleation centers of the new phase can be imaged, allowing their growth and coalescence to be documented. Second-order phase transitions will provide another level of insights as fluctuations grow in spatial extent and undergo critical slowing down as the transition is approached and crossed. Using ultrafast electron microscopy, the internal space and time structure of the thermodynamic scaling laws can be directly observed for the first time.

Pump-probe ultrafast electron microscopy experiments can access much of the fluctuation regime, monitoring the electronic, lattice, and magnetic degrees of freedom; distinguishing thermal from quantum fluctuations; and tracking the nucleation and growth of new phases. Fundamental questions include:

- Can the electronic, lattice, and magnetic components of the fluctuation spectrum be separately resolved in time, space, and energy?

- Can energy transduction between electronic, lattice, and spin degrees of freedom be quantified?

- How is energy transduction impacted by external electromagnetic fields?

- When is a dynamic picture necessary versus a simple statistical one?

In contrast to existing probes, such as STM and angle-resolved photoemission spectroscopy, ultrafast electron microscopy probes the material's bulk properties. Therefore, spurious effects due to surface construction are of less concern. Furthermore, for UTEM experiments demands on the surface quality are strongly relaxed, opening access to much broader families of materials.

\subsubsection{Radiation Damage}

\subsubsection{Radiation Damage in Nanostructured Ferritic Steels}

The renewed emphasis on nuclear power has triggered interest in a new class of nanostructured ferritic steels (NFS) now being developed for next-generation nuclear power plant applications. ${ }^{174}$ Nanostructured ferritic steels have the potential for a stable microstructure compatible with operation at high temperatures $\left(>700^{\circ} \mathrm{C}\right)$ and under high doses of irradiation (up to $100 \mathrm{dpa}$ ). ${ }^{175-180}$ In NFS, the key microstructural features are complex Ti-Y-O clusters (Figure 31), which are especially effective in limiting grain growth and the mobility of dislocations at high temperatures, ${ }^{175,180}$ allowing excellent 
high temperature mechanical properties. ${ }^{179}$ In addition, high surface areas of these clusters are potentially powerful trapping sites for transmutation-produced He atoms, which is a highly desirable property in nuclear fusion applications.

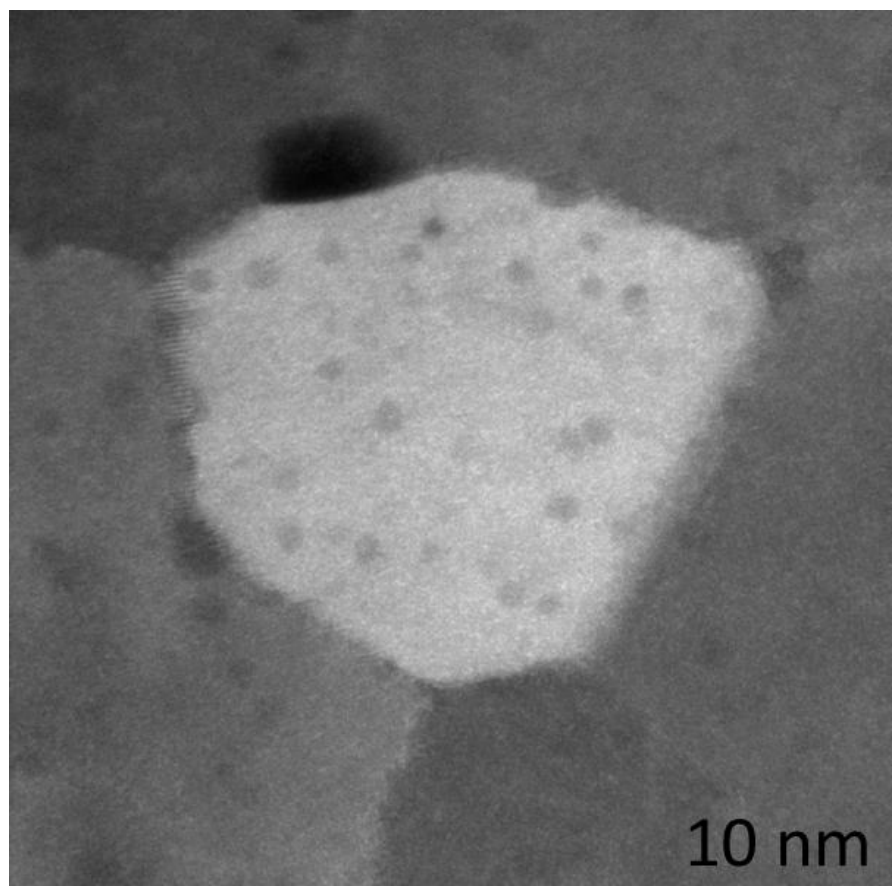

Figure 31. Fine grain microstructure of NFS with a high density of Ti-Y-O-rich nanoclusters. ${ }^{181}$

Detailed understanding of the composition and structure of the Ti-Y-O clusters, their resistance to radiation damage, and their ability to trap radiation-induced point defects is critical for future atomic-scale design and optimization of NFS materials. While conventional TEM has been extensively used as a tool to study the nature of nanoclusters, it does not have the time resolution required to capture the mechanisms associated with the actual primary knock-on atomic displacements, radiation-induced amorphization, dissolution, or reprecipitation. The time resolution afforded by ultrafast electron microscopy could illuminate the dynamics of these radiation processes. For example, the time-scale for the radiation-induced dissolution of the nanoclusters is expected to be on the order of a few picoseconds, a time-scale that also applies to high-energy neutrons generating primary recoiling atoms (at energies of up to several hundred keV) and subsequent branching cascades with additional recoiling atoms displaced from lattice sites. ${ }^{182}$

Capturing the sequence of events involved in the dissolution of nanoclusters of various sizes and structures would reveal the relationships between structure, size and stability and provide fundamental insights into the mechanisms that govern this relationship. It has been previously shown that a range of composition and interfacial structures for Ti-Y-O clusters ${ }^{174}$ exists, and that a core-shell structure with Ti segregation at the interface is expected to produce excellent strength and radiation stability. This hypothesis could be directly tested with ultrafast electron microscopy. The mechanisms of point defect trapping at the interfaces of nanoclusters and other precipitates are another important topic in radiation damage in NFS. This process occurs at a slower rate than the few picoseconds of the displacement cascade, well within the realm of imaging by ultrafast electron microscopy. Imaging the trapping events and the associated atomic configurations at the coherent and incoherent interfaces of the relevant clusters and nanoscale $\mathrm{Y}_{2} \mathrm{O}_{3}, \mathrm{Y}_{2} \mathrm{TiO}_{5}$, and $\mathrm{Y}_{2} \mathrm{Ti}_{2} \mathrm{O}_{7}$ precipitates would represent a substantial advancement that could provide insights into the balance between the rate of primary defect generation, such as knock-on displacements, and derivative defect formation such as void and other cluster formations which destabilize the microstructure. 


\subsubsection{Damage Initiation and Collision Cascade Analysis}

\subsection{Defect Annealing Studies}

Radiation damage processes in metals, alloys, ceramics, and biological materials are of paramount importance for nuclear plant safety and future energy needs. Radiation damage can cause hardening and embrittlement; swelling and volumetric shape changes; impurity segregation leading to grain boundary chemistry changes; nonequilibrium phase formation; amorphization in complex crystal structures; and a host of physical and chemical changes in material properties. Damage initiation processes have been widely studied using molecular dynamics simulations with semi-empirical interatomic potentials. However, the ultrafast time scales of these processes so far have eluded experimental study in imaging mode. A collision cascade in a pure metal is a complex event, depending on the initial kinetic energy of the primary knock-on atom (PKA) that can displace several thousand atoms in a few ps of ionization energy loss. Figure 32 shows a simulation of the extent of a $200 \mathrm{keV}$ collision cascade in Fe at the peak of the damage (Figure 32a) at $0.67 \mathrm{ps}$ and then at the end of the damage event at approximately $14.5 \mathrm{ps}$ (Figure 32b). UTEM studies would be very useful toward understanding the extent and nature of this primary damage event. Although damage initiation is clearly demarked the end of the cascade, it is a bit nebulous and typically consists of several freely migrating defects moving by diffusion in the almost perfect lattice that has been largely restored by recombination events. ${ }^{182}$
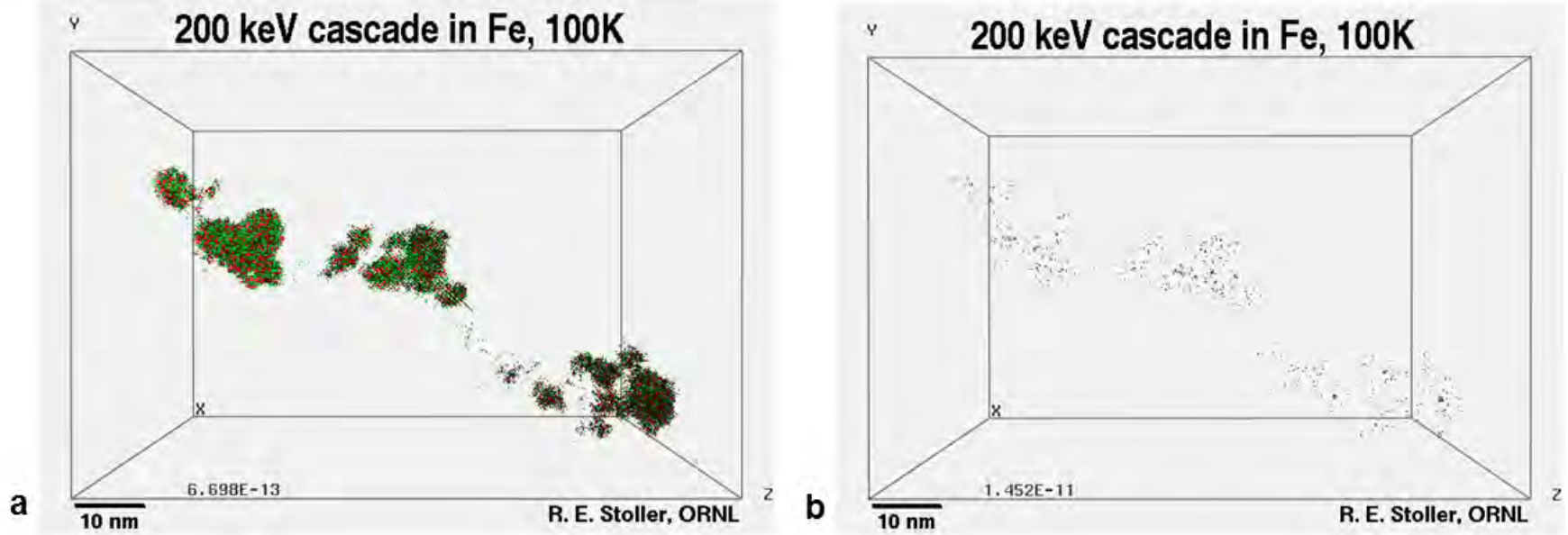

Figure 32. Molecular dynamics visualization of a $200 \mathrm{keV}$ collision cascade in pure Fe showing the peak of the damage at $0.67 \mathrm{ps}$ (a) after the initiation of the damage and near the end of the cascade at $14.5 \mathrm{ps}$ (b). The defects seen in (b) are representative of the freely migrating defects produced by the cascade that determine the extent of the radiation damage. For additional movies documenting radiation damage processes, refer to http://www-personal.umich.edu/ gsw/movies.html. ${ }^{182}$

These initial damage events differ for alloys and ceramics because atomic displacements for each element might be different, and, particularly for ceramics, amorphous regions might be produced where the crystalline structure is lost completely as shown in Figure 33. The formation of such amorphous regions is typical of covalent- or ionic-bonded materials in non-close-packed structures and reduced atomic mobilities relative to metals and alloys.

In each case, the scientific interest resides in capturing the details of the initial damage event as to its spatial extent and atomistic details of the damage formation. The time scales appear to be well suited for UTEM image capture. The event trigger would be the incoming ion or charged particle that causes the damage cascade. Of course, this considers the case where an ion beam station or small accelerator is linked to the UTEM system to allow the damage event to occur. Cascade 
overlap, where two or more cascades occur in overlapping or adjacent volumes, is a current area of study in radiation damage that could be examined in this manner. Inert gas injection, such as He, also is of keen scientific interest in this field in terms of gas bubble formation in irradiated solids.

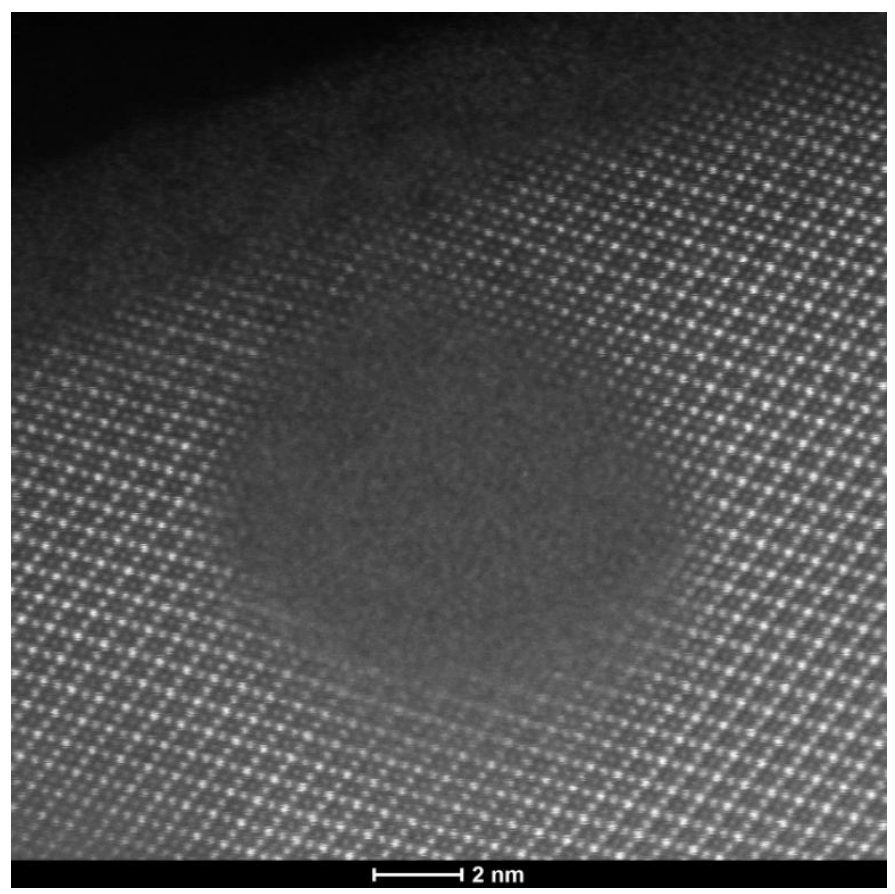

Figure 33. High-energy heavy ion damage in $\mathrm{Gd}_{2} \mathrm{Ti}_{2} \mathrm{O}_{7}$ showing several nm-sized amorphous regions perpendicular to the ion track (into the photo). Amorphous regions are typical of damage in covalent or ionic materials. Note the lack of damage and disorder at the edge of the amorphous region. ${ }^{181}$

An important complementary study to damage initiation would be careful studies of damage annealing in materials. An external trigger, such as a laser, initiates the damage annealing process and, with appropriate delay, the electron probe of the state of annealing. For example, the sample shown in Figure 33 could be annealed and imaged using UTEM images observe the recrystallization from the amorphous region. There might be interesting similarities to a solid-liquid system that could be explored.

\subsubsection{Flow Localization via Dislocation Avalanche Formation}

The lower operating temperature limit for most structural materials in the fusion neutron environment is determined by radiation hardening and embrittlement. At irradiation temperatures less than $\sim 30 \%$ of the absolute melting point, the defect structures produced by neutron-induced displacement damage significantly increase tensile strength, decrease ductility, and can lower fracture toughness. Although the experimental parameters that cause radiation hardening and embrittlement are well known, the fundamental mechanisms responsible for loss of ductility are not fully understood. Improved multiscale models coupled with appropriate experiments are needed to determine the causes of ductility loss in irradiated metals and to suggest possible metallurgical solutions to ameliorate this effect.

Low-temperature neutron irradiation produces a high number density of nanometer-scale defect clusters that interfere with the nucleation and motion of dislocations. Microscopically, plastic deformation is confined to narrow ribbons of material, typically a fraction of a micron wide (Figure 34). This phenomenon, known as "dislocation channeling" since most of the 
dislocation activity occurs in these narrow bands, is a common strain localization mechanism observed in virtually all irradiated metallic materials.

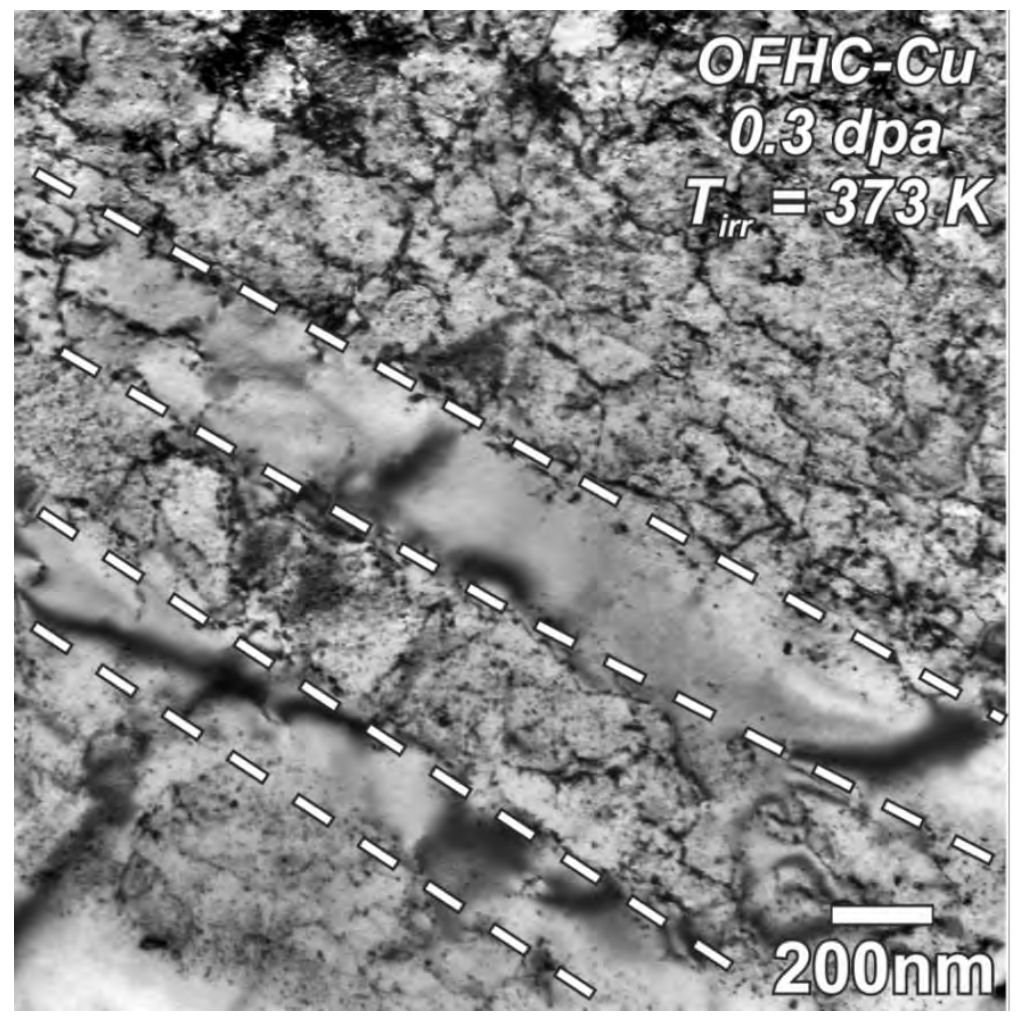

Figure 34. Cleared channels formed in oxygen-free high-conductivity copper. ${ }^{183}$

The formation of dislocation channels in neutron irradiated metals and alloys has been extensively reported in the radiation effects literature for many years. Despite the considerable body of experimental observations and computational modeling studies, the detailed mechanisms of channel formation are largely unknown. Most studies have confirmed that the irradiation-induced defect clusters are either partially or fully cleared by gliding dislocations within the channels. The observations also strongly suggest that channels are initiated at grain boundaries, particle-matrix interfaces, and at previously formed channels. These regions are preferred locations for dislocation nucleation because they provide stress concentrations that allow dislocations to overcome the long-range elastic interactions with the irradiation-induced defect clusters.

UTEM could provide valuable insights into the dynamic deformation processes at work in these materials. It is hypothesized that very high local stresses, such as that existing at the head of a dislocation pile-up, are needed to overcome the inhibiting effects of the obstacle field. Homogeneous nucleation of dislocations seems unlikely because stresses on the order of the theoretical strength of the solid would be required. Consequently, stress concentrators must play a role to reduce the required applied stress to levels observed in experiments. Finite element simulations indicate that elastic anisotropy and grain boundary steps are relatively weak stress concentrators. On the other hand, extrinsic grain boundary dislocations with localized cores are far more powerful stress concentrators. Obstacles to slip in grain boundaries, such as steps, precipitates, or chemical segregation, provide mechanisms to promote extrinsic grain boundary dislocations, core localization, and dislocation emission into the matrix. Because of the high stresses involved, emission of an avalanche of dislocations is presumed to occur from a very small volume of material at a very high rate. The situation is quite similar to Lüders band formation in low-carbon steels. To elucidate the role of stress concentrations within grain 
boundaries, the high spatial and temporal resolution offered by UTEM will be essential for capturing these events in sufficient detail.

\subsubsection{Defects in Graphene Films}

Graphene is a unique, two-dimensional material because of its low concentration of intrinsic defects and the high mobility of synthetic defects that can be artificially introduced. The high mobility of artificial defects is critical to the targeted functionality of graphene devices. ${ }^{184}$ The absence of an electronic bandgap in pristine graphene precludes its use as a transistor or in other nanoelectronic applications. Despite their importance for applications, the influence of the high mobility and mutual interaction of defects on the electronic behavior of graphene is poorly understood. The dynamical behavior and attraction or repulsion of defects likely will have profound influence on macroscopic properties. Ultrafast time resolved imaging of transient states is critical toward understanding the dynamical behavior of structural defects and the functionality it confers.

The reduced dimensionality of graphene restricts the number of defect types. Allowed defects include point defects such as vacancies or interstitial atoms and dislocations (one-dimensional lines of defects). A simple example is the Stone-Wales defect that involves a $90^{\circ}$ rotation of a single bond, resulting in the transformation of four hexagons into two pentagons and two heptagons. This defect has a formation energy of $5 \mathrm{eV}$, an energy barrier to formation of $\sim 10 \mathrm{eV}$, and a reverse energy barrier of $\sim 5 \mathrm{eV}$. While the high formation energy implies a negligible equilibrium concentration, once the defect is formed under non-equilibrium conditions, the high energy of the reverse transformation guarantees its stability at room temperature. Note that such transformation is possible even when the electron beam transfers less than the threshold energy for atomic displacement $(\sim 20 \mathrm{eV})$ to carbon atoms because bond rotation requires less energy than a knock-on displacement.

Single vacancies are a common defect in graphene and are frequently observed using TEM and STM. The single vacancy often is accompanied by a Jahn-Teller distortion that reduces the number of dangling bonds to two by creating a pentagonal ring. The resulting defect produces localized electronic states at the Fermi energy. The formation energy for this defect is calculated to be $\sim 7.5 \mathrm{eV}$, while its predicted migration barrier is $\sim 1.3 \mathrm{eV}$. Such a barrier is sufficiently small to permit the measurement of migration at temperatures only slightly above room temperature. To date, conventional techniques have not directly observed the migration of point defects in graphene, even though the mobility of these defects is frequently invoked to explain the observed annealed or reconstructed configurations under the stress of electronic irradiation. Imaging of the motion of graphene defects by UTEM is critical toward understanding their behavior and role in creating unique functionality for nanoelectronic devices. 


\section{Instrument}

\subsection{Design and Implementation of an Ultrafast Transmission Electron Microscope}

The scientific Grand Challenges discussed in this report - the key drivers behind the design and implementation of a new ultrafast transmission electron microscope (UTEM) — cover a wide range of spatio-temporal regimes-from microseconds to femtoseconds and from $0.1 \mathrm{~nm}$ to $10 \mathrm{~nm}$. In addition, to fully realize the potential for the UTEM to revolutionize our understanding of many areas of science and technology, one of the key spectroscopic methods in conventional transmission electron microscopes - electron energy loss spectroscopy (EELS) — also must be implemented. In this case, the energy resolution needed to be obtained goes well beyond the $100 \mathrm{meV}$ resolution of the best conventional field emission microscopes equipped with monochromators and must approach $10 \mathrm{meV}$ resolution. While many of these capabilities have been demonstrated in the groundbreaking ultrafast electron microscopy work of Zewail and co-workers, ${ }^{10,172,185-187}$ their results have been obtained in a stroboscopic method that requires the system being studied to be perfectly reversible. The goal of this section of the workshop report is to define the electron-optical components for a UTEM that is primarily capable of obtaining ultrafast images in the single shot mode of operation - i.e. a full image can be obtained from a single laser pulse interacting with the photocathode. It is only through this type of microscope that the majority of the science described in this report can be achieved. As the ability to achieve resolution in stroboscopic mode has already been demonstrated, this capability will also be included as part of the UTEM concept (the addition of an ultrafast laser will be all that is needed to include this functionality).

\subsection{Obtaining Temporal Resolution in TEM with a Photo-Emission Source}

Time-resolved experiments are performed in a conventional TEM by using a fast framing camera to essentially acquire "snapshots" of the sample. ${ }^{188-192}$ The limitation to this type of experiment comes from the number of electrons needed to form a high quality image. As a field emission source emits electrons in a constant stream, and the camera limits the number of these electrons that can hit the detector by having a short exposure time at some point the detector does not receive enough electrons to form an interpretable image. For a field emission microscope this limit is $\sim 25 \mathrm{~ms} .{ }^{27} \mathrm{~A}$ better way of achieving high temporal resolution is to bunch the electrons up so that they are only emitted over a certain short time interval with the beam being off for the other times. One of the simplest means of obtaining this type of electron "bunch" or "pulse" is to use a short pulse laser to create a pulse of electrons through the photoemission process. By illuminating a metal disc with a laser of energy above the work function of the metal (typically in the ultraviolet (UV) range of the optical spectrum), a very short pulse of electrons can be created that will have approximately the same duration as the laser pulse. This approach removes the limitation in the detector from the ability to achieve high time resolution and makes the "source laser" the major determining factor for temporal resolution-where laser pulses on the femtosecond timescale are now routinely available.

Time resolved experiments are conducted by first initiating a transient state in the sample and then taking a snap-shot of the transient process with the short electron pulse at some preferred time delay after the initiation. In most experiments, the transient process is initiated with a laser pulse that enters the TEM column through a modified port (usually the x-ray port). For nanosecond time-scale experiments, neodymium-doped yttrium aluminum garnet (YAG) lasers can be used as the "specimen drive" with a pulse duration from 3 to $25 \mathrm{~ns}$ that can produce fluences up to $1500 \mathrm{~J} \mathrm{~cm}^{-2}$ on the specimenhigh enough to turn most specimens into a plasma. By controlling the laser energy and spot on the sample, a wide range of temperatures and heating rates can be produced in the sample. The fundamental wavelength $(1064 \mathrm{~nm})$ of these lasers can 
be frequency converted using nonlinear harmonic generation crystals, e.g., doubled (532 nm), tripled (355 nm) or quadrupled $(266 \mathrm{~nm})$, as dictated by the absorption characteristics of the sample and the desired experimental conditions. For instance, metals have broadband absorption. Thus, all of these wavelengths can be used, while certain semiconductors only absorb sufficient amounts of laser energy in the visible or UV range and may require frequency doubled or tripled laser pulses. A schematic of the set-up for this laser mediated, time-resolved microscopy and the Dynamic Transmission Electron Microscope (DTEM) at Lawrence Livermore National Laboratory (LLNL) are shown in Figure 35.
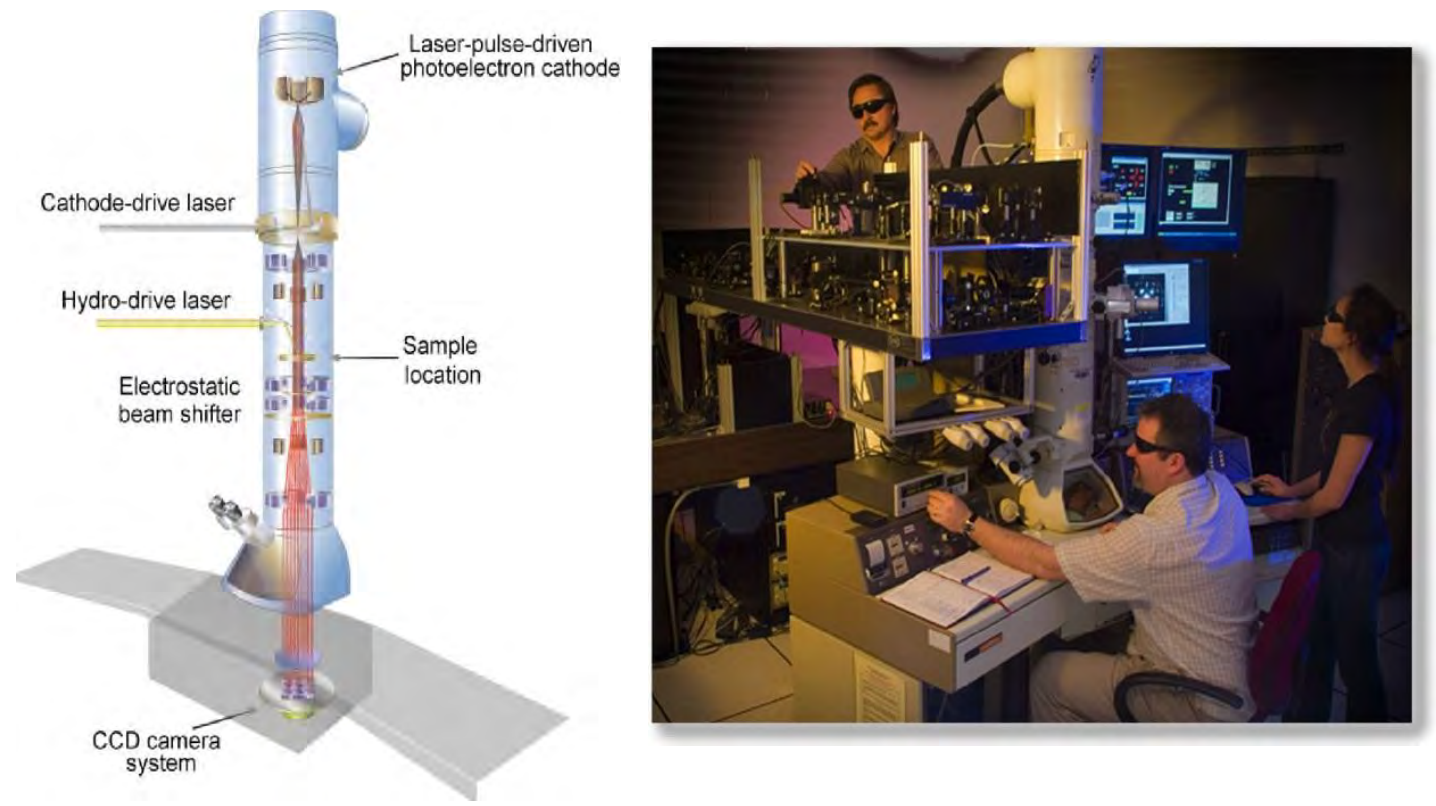

Figure 35. Schematic of the LLNL DTEM I (left) and (right) image of the LLNL DTEM I as installed. ${ }^{29,30,193-196}$

\subsection{Two Contrasting Modes of Operation: Stroboscopic and Single Shot}

Within the photoemission framework, there are two methods for obtaining TEM images with fast time resolution. The first approach, as mentioned above, was pioneered by Zewail et al. and uses a femtosecond source and specimen laser to achieve its resolution. ${ }^{10,172,185-187}$ The key to the method is to keep only a single electron in the column at any one time to reduce space-charge effects. Images are built up from $\sim 10^{8}$ of these single electron shots that have been precisely correlated with the specimen drive laser. The single electron per pulse method means optimal temporal and spatial resolution of the microscope can be maintained (the operation is similar to a conventional microscope but each electron that arrives at the detector has a time registry associated with it. However, the fact that the specimen must be pumped $\sim 10^{8}$ times by the laser means that the process being studied must be perfectly reversible - the sample must heal between shots. This means that the highest time and spatial resolution is obtained for the study of such effects as molecular interactions, atomic motions, and electronic phase transitions (these types of phenomena are discussed in this report and we will return to the stroboscopic mode of operation in the discussion of laser design for UTEM).

The second approach was pioneered by Bostanjoglo and co-workers and aims to acquire single shot images using large pulses that contain enough electrons to generate sufficient image contrast. ${ }^{28,197,198}$ The single shot approach means that the process being studied need not be perfectly reversible as all the information is obtained from a single specimen drive event. However, the limitation to this method is that space-charge effects in the beam can lead to degradation of resolution and even with an optimized microscope source, column, and detector the high current will limit the overall temporal and 
spatial resolution of the instrument. The key to using this single shot approach is therefore to optimize the components in the microscope to define the space-charge limited resolution of the instrument. It is this approach that has been employed at LLNL using a modified JEOL 2000FX (Figure 35) that now achieves $\sim 10 \mathrm{~nm}$ spatial resolution at a time resolution of $\sim 15 \mathrm{~ns}$ (Figure 36 and Figure 37). The lessons learned from the LLNL DTEM ${ }^{29,30,193-195}$ form the basis of the DTEM II design at UC-Davis and both instruments will aid in the design and implementation of a UTEM described here.
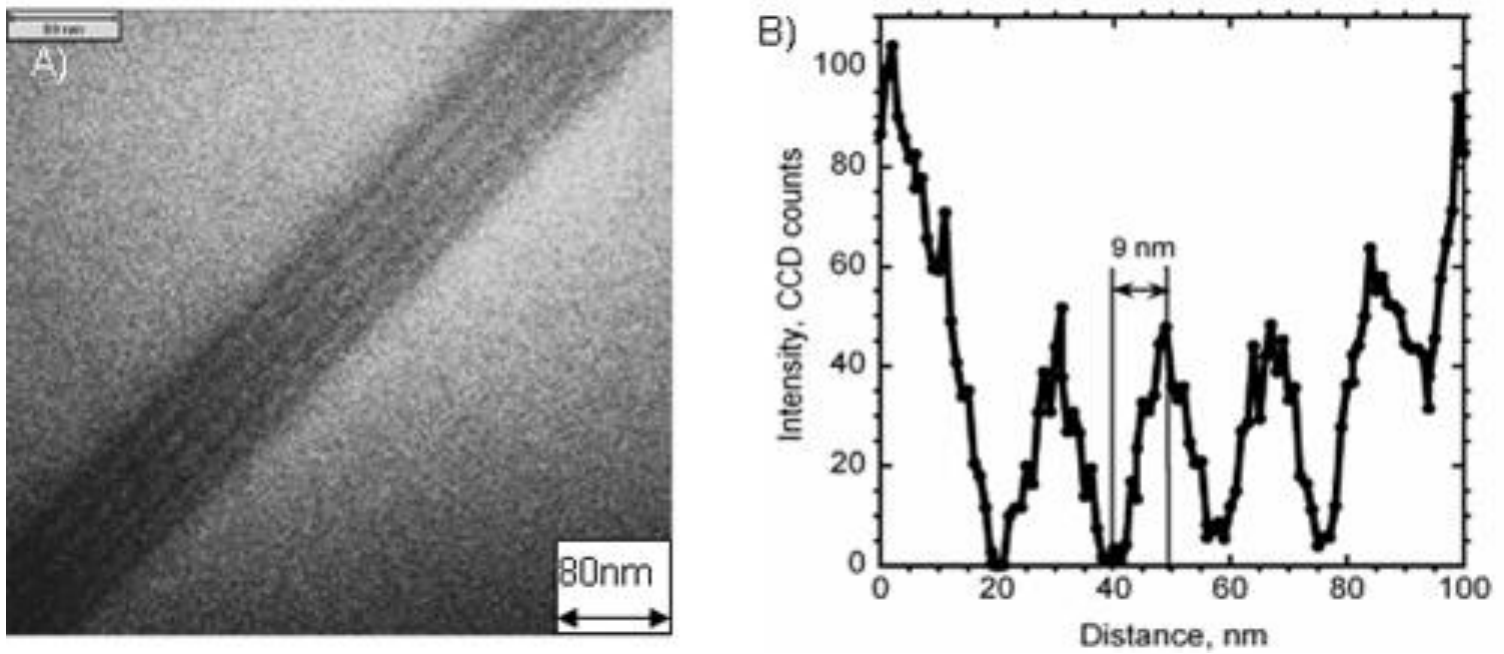

Figure 36. Single-shot, 15-nanosecond image of an Au/C grating obtained from LLNL's DTEM I showing resolution less than $10-\mathrm{nm}^{193}$.

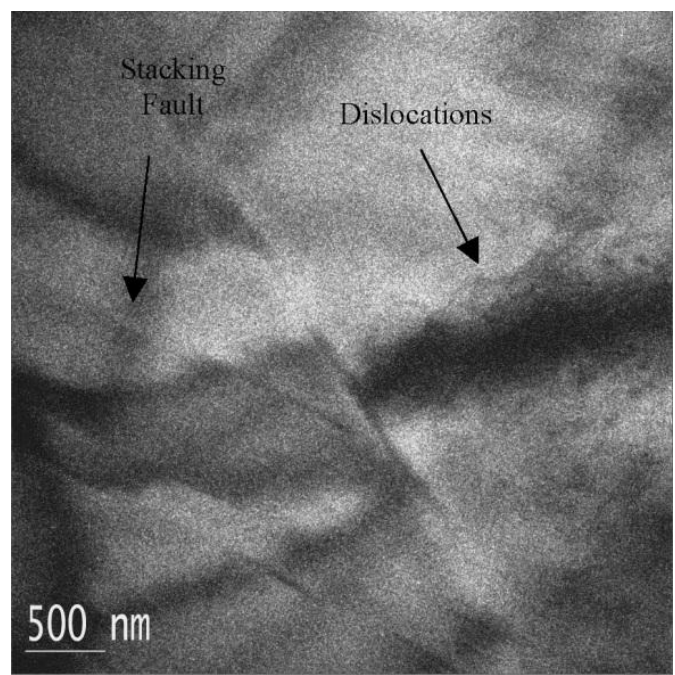

Figure 37. This single-shot pulsed image of a stainless steel sample clearly shows that LLNL's DTEM I can image material defects such as dislocations and stacking faults with a single 15-nanosecond exposure. $^{196}$

\subsection{Resolution Limitations for DTEM}

The implementation of a single-shot dynamic TEM and the potential for us to move towards a UTEM starts with a physical understanding of the electron pulses themselves (described in detail in ${ }^{195,196}$ ). Because of the need to extract 
information from the sample at an extraordinary rate (measured in bits of information per square nanometer per nanosecond) compared with conventional TEM, a single shot DTEM must operate at extremely high current densities while at the same time preserving the spatial and temporal coherence of the beam. This is due to the physics of TEM image contrast, including the electron-sample interaction and the electron-optical properties (including aberrations) of the lens system. The first stage of improving the performance of a UTEM is therefore the optimization of the electron optical system for controlling the electron pulses when they leave the gun.

Ultimately, the performance of a DTEM (and subsequently the UTEM described in later sections) is governed by the brightness of the electron source. While this is true for any TEM, it is especially true for a single-shot instrument that demands a very high fluence (electrons per unit area) be delivered in a very short time. We will define brightness B as

$$
B=\frac{N e}{\left(\pi r^{2}\right)\left(\pi \alpha^{2}\right) \Delta t},
$$

where $\mathrm{N}$ is the number of electrons per pulse, $\mathrm{e}$ is the electronic charge, $\mathrm{r}$ is the radius of the electron beam, $\alpha$ is the local convergence semi-angle, and $\Delta t$ is the time duration of a pulse. B is very nearly conserved for a beam propagating at a fixed accelerating voltage. By "nearly conserved," we mean that B is a constant in an ideal electron column, but in real systems a number of physical effects (e.g. Boersch effects, ${ }^{199}$ aberrations, and space charge effects ${ }^{200}$ ) can cause the effective brightness to degrade with propagation distance. If the voltage is not constant, then the normalized brightness (equal to fundamental constants times $\lambda^{2} B$, with $\lambda$ the electron wavelength ${ }^{201}$, and using the relativistic deBroglie relation $\lambda=h /(\beta r m c))$ is the relevant conserved quantity that includes all relativistic corrections. The quantities $r, \alpha$, and $\Delta t$ are finite and the brightness as we have defined it is an effective average over a finite area. This definition has the advantage of direct experimental relevance, but as a result our $B$ is not a precisely conserved quantity in a freely propagating beam; lens aberrations, space charge, and statistical Coulomb effects can all reduce our effective average $B$ as a beam propagates through a column. This will become more prominent for the sub-picosecond regime of the UTEM described later, in which pulses will expand longitudinally so that $\Delta t$ is no longer constant. ${ }^{202-205}$ The goal in establishing an electron-optical column for all time resolved single-shot studies is therefore to incorporate a flexible set of components that minimizes the degradation of the beam as it propagates along the column.

We can also define the related quantity, transverse emittance, as $\varepsilon_{T}=r \alpha$, which is itself a nearly-conserved quantity just as $B$ is, provided no electrons are blocked at apertures. A smaller $\varepsilon_{T}$ implies a higher-quality beam, with higher spatial coherence for a given spot size. Lenses can transform the phase-space occupied by electrons so long as the phase-space area doesn't change. If the lenses are aberration-free, then a phase-space ellipse remains an ellipse throughout the column, even though its tilt and aspect ratio can change. Multipole lens elements such as those in aberration correctors can perform more complex operations and in principle, a sufficiently complex lens system could allocate this phase-space area in just about any desired manner (which, for most experiments, would be an ellipse at the sample, though some results from light optics suggest other possibilities ${ }^{206}$ ). In time-resolved electron microscopy, we also need to consider the longitudinal phase space, which shows the distribution of electron speeds (or energies) as a function of arrival time (or of longitudinal position) relative to the center of mass of the bunch. The combination of this longitudinal phase space with the transverse phase spaces in the $\mathrm{x}$ and $\mathrm{y}$ directions comprise a six-dimensional phase space. Longitudinal phase space area is also approximately conserved and can be manipulated with various combinations of space-charge expansion, ballistic propagation, and pulse compressors (which we may think of as temporal lenses). This is the basis of existing proposals to improve the temporal resolution of UED while still minimizing the energy spread ${ }^{207}$.

The longitudinal space charge effects that cause $\Delta t$ to increase as a pulse propagates are only very weakly coupled to the aberrations that cause the transverse emittance $\varepsilon_{T}$ to degrade (although they do affect the rate of evolution of the lateral 
phase space ellipse). ${ }^{202-205}$ Thus the product $B \Delta t$ (which is proportional to the number of electrons in a pulse) is also nearly conserved. Combining this effect with the variation of brightness with accelerating voltage, we find that the dimensionless quantity:

$$
N_{C} \equiv \frac{\pi^{2} \lambda^{2} B \Delta t}{e}
$$

(which we will call the coherent fluence) is a convenient figure of merit that is very nearly constant as a function of propagation distance for any electron pulse we would likely be using. This definition is motivated by a recognition that the lateral coherence length is given by $r_{C}=\lambda \alpha$, so that our quantity $\mathrm{N}_{C}$ is essentially the number of electrons per lateral coherence area, per pulse (to within definition-dependent factors of order unity). No amount of lensing, aperturing, acceleration, or space charge dynamics will allow a user to significantly improve this value once the pulse has left the gun (see later section on gun design). As can be shown, the coherent fluence plays a central role in the theory of resolution limits for single-shot pulsed imaging. For example, if $\mathrm{N}_{C}$ is not much more than 1, then coherent single-shot imaging is impossible, no matter how good the lens system is. It is for this reason that the gun will be the key element in the design of the UTEM discussed in the next section.

To understand where the limits of resolution in the DTEM lie, we can consider the interplay of brightness, coherence, shot noise, and the instrumental contrast transfer function, with the goal of determining the spatial resolution limit as a function of time resolution $\Delta \mathrm{t}$. It is important to note that these calculations are in the spirit of an order-of-magnitude estimate for the performance as limited by the microscope itself; we assume an ideal sample with $100 \%$ contrast arising from the electron-sample interaction (which can be achieved in practice through the use of a phase plate). Post-specimen space charge and stochastic blurring effects are not included in this order of magnitude estimate as the calculations depend sensitively on the operating conditions of the microscope being used and it is impossible to cover all the possibilities. The important thing for DTEM and UTEM is that the column has the flexibility in electron optics to test all these parameters and define the optimum for resolution.

Standard textbook formulae can be used for a conventional TEM's partially coherent contrast transfer functions $(T(r))$ for both phase and amplitude contrast, ${ }^{208}$ including spatial and temporal incoherence effects through the usual envelope approximations. Assuming that the microscope user is interested in getting the best possible contrast at a given spatial frequency, with a fixed brightness and single-shot time resolution, which means (1) adjusting the objective lens defocus to maximize $|T|$ for the desired spatial frequency band (this is done implicitly for all the results shown here), and (2) increasing the current density by converging the beam until $\alpha$ is large enough that the spatial incoherence is starting to significantly reduce $|T|$. We define a coherence factor $f_{\text {coherence }}=\lambda / \delta x \theta$, with $\lambda$ the electron wavelength, $\delta x$ the radius of the smallest feature to be resolved, and $\theta$ the half-width of the angular distribution function (convergence angle of the lens). In other words, we have scaled the pixel size to the lateral coherence length via the dimensionless factor $\mathrm{f}_{\text {coherence. }}$. Employing the above definition of brightness, we find that the number of electrons per pixel area $\pi r^{2}$ is

$$
N=\frac{\pi^{2} \lambda^{2} B \Delta t}{e} \cdot \frac{1}{f_{\text {coherence }}^{2}}=\frac{N_{C}}{f_{\text {coherence }}^{2} \text {. }}
$$

The coherent fluence has come up naturally from the imposition of our condition (2). We can now apply the Rose criterion, ${ }^{209}$ which specifies the minimum number of particles that need to be detected before a pixel can be said to be resolved in the presence of shot noise. The governing formula is $N^{1 / 2}=|T| / f_{\text {Rose, }}$, with $f_{\text {Rose }}$ typically set to $\sim 5$ and $T$ is the contrast transfer function. Combining equations yields the formula 


$$
N_{C}=\left(\frac{f_{\text {coherence }} f_{\text {Rose }}}{T}\right)^{2} \text {, }
$$

where the coherent fluence is set equal to a combination of dimensionless parameters. The absolute contrast transfer function $|T|$ is maximized with respect to defocus at the spatial frequency of one cycle per $2 \delta x$ (where $\delta x$ is the spatial resolution). We can also minimize the required $N_{C}$ as a function of $\alpha$, but the inverse-linear dependence of $f_{\text {coherence }}$ on $\alpha$ more than balances the relatively weak $\alpha$ dependence of our approximate transfer function $T$, suggesting that the optimum is at extremely large convergence angles. However, the standard formulae for the spatial coherence envelope come from a first-order approximation, and they break down at large $\alpha$, so that the experimentally-relevant optimum may be closer to $f_{\text {coherence }}=1$. In concrete terms, converging the electron beam increases the signal-to-noise ratio very quickly, but after a certain point the spatial incoherence rapidly deteriorates (in a way that isn't captured by the basic equations) so that no imaging is possible. As a practical compromise, we match the coherence length to the target resolution, i.e. we set $f_{\text {coherence }}=1$, for all coherent imaging modes.

From these simple formulae, we may conclude a number of things, keeping in mind that the models employed are intended for rough estimates only. First, the current DTEM at LLNL should be capable of incoherent imaging on the scale of $\sim 10 \mathrm{~ns}$ and a few nm with the use of an ideal 100\%-contrast sample. At present, the DTEM achieves better than 10-nm resolution in 15-ns exposures in conventional imaging, ${ }^{29,30,193-196}$ with real samples that inevitably have less than $100 \%$ contrast. Thus, the calculations seem to be reasonably close to reality in this indirect comparison. Increasing the brightness to be comparable to that of a field emission gun (FEG) would enable nearly the same performance at $10 \mathrm{~ns}$ as would be possible with the current brightness at $1 \mu \mathrm{s}$, while an equivalent FEG brightness at $1 \mu$ s should be capable of atomic resolution imaging over a wide spatial bandwidth. $\mathrm{C}_{\mathrm{s}}$ correction would allow imaging to push down to angstromscale resolution, where the chromatic aberration becomes the limit according to present models (this is the goal for DTEM II). In principle, the addition of a phase plate, which, for small convergence angles, allows the user to swap the coherent amplitude and phase contrast transfer functions, to an aberration-corrected, high-brightness system could enable atomic resolution at faster timescales, provided the electron-electron scatter can be minimized.

\subsection{The Evolution from DTEM to a True Single-Shot UTEM}

The main goal of the technology described herein is to implement the single-shot approach to dynamic observations in the TEM in the ultrafast regime, i.e., develop a microscope that can operate on the sub-ns timescale. To ensure that this instrument is applicable to as many different scientific challenges as possible, a second design constraint is to ensure that for timescales below the single-shot limit, the microscope can operate as a stroboscopic instrument and achieve the same resolution as shown by Zewail and co-workers ${ }^{10,172,185-187}$. By seamlessly changing to stroboscopic mode, this allows the instrument development path (see later) to consistently work towards improving combined spatiotemporal resolution while not degrading any other aspect of the performance. The final design constraint for the microscope is the ability to perform high resolution EELS with an energy resolution that is $\sim 10 \mathrm{meV}$. This type of energy resolution is an order of magnitude improvement over conventional microscopes and a worthy goal in its own right. When coupled with the temporal resolution of the UTEM, this will allow electronic transitions in superconductors, for example, to be studied directly. This level of resolution also will permit phonons to be studied by electron spectroscopy. 


\subsection{The Column}

The discussion of the limitation to resolution in a single shot instrument that are discussed in section 3.4 make the design path for the electron microscope column self-evident. The microscope column must have a flexibility in the lens configurations that allows the beam to be manipulated along the column to preserve the coherent flux. Any degradation in the beam path caused by aberrations, for example, will degrade the ability to obtain the highest spatial resolution images at a given pulse duration. This design path has actually been followed by all the previous single shot instruments that have been constructed. As shown in Figure 38, the initial work on single-shot imaging was performed by Bostanjoglo et al. ${ }^{28,197}$ using a Siemens Elmiskop (a 1950's vintage TEM). The next generation instrument built at LLNL, moved to more modern lens systems (lower aberrations) in a JEOL 2000 FX microscope (a 1970's vintage TEM). This upgrade in electron optics realized two orders of magnitude improvement in the spatial resolution for the same temporal pulse. A more recent DTEM II being developed at University of California, Davis (UC-Davis), incorporates two aberration correctors (one a pre-specimen and the other a post-specimen) corrector and an in-column filter (for energy filtered imaging and EELS) in a JEOL 2200 microscope (a 2000's vintage microscope). This microscope also improved the gun geometry to improve the brightness (see section 3.7) and is expected to again deliver an order of magnitude increase in spatial resolution at a given temporal pulse length.

Also shown in Figure 38 is a design for the column for a UTEM which will build on the components included into the UC-Davis DTEM. In this case the electron optics incorporates the developments that have taken place over the last 5 years, many of which were supported by the Department of Energy through the TEAM project ${ }^{210}$ - the column incorporates two Cs and one Cc corrector (the Cs correctors are already being implemented on the UC-Davis DTEM), an in-column filter (already being implemented on the UC-Davis DTEM), a phase plate (either a conventional design or the laser initiated design with Patent pending from LLNL), an RF pulse compressor (being implemented at the University of Illinois at Chicago and by others for UTEM applications) and a frame shifter to obtain "movies" of dynamic events (this is already in the process of being implemented on the LLNL DTEM I). The interesting aspect of this column design is that apart from the RF cavity for pulse compression and the frame shifter, this is the same optimized column you would design for high spatial resolution microscopy. As the RF cavity is small (a few centimeters in length) and can be included into the top of the $\mathrm{C}_{\mathrm{s}}$ corrector and the frame shifter is an external power supply that utilizes existing deflection plates in the microscope, we are seeing a convergence of the technologies for single-shot time resolved microscopy with high resolution microscopy. While the components of the system will be operated in a different manner to take into account the intense pulses of electrons in the UTEM, this design for the column means that the only change needed to move from a high resolution instrument operating with a field-emission source to a UTEM operating with a photoemission source is a replacement of the gun (the UC-Davis DTEM II will implement all the components for the photoemission source within an existing field emission gun itself).

The optimized and flexible electron column described above means that the limitation in resolution for a UTEM is controlled by the gun. To move into the ultrafast sub-ns time regime it is therefore essential to define the operating parameters of the gun. Once this column is built, all future advances in the spatial resolution obtained for a given pulse duration will be tied to the brightness of the gun - whatever the limitation for resolution turns out to be in the electron optics for a medium voltage microscope, the only way to utilize that resolution limit will be through an optimized photoemitter. For this reason, a focus of any UTEM project to improve spatio-temporal resolution will be the gun. 


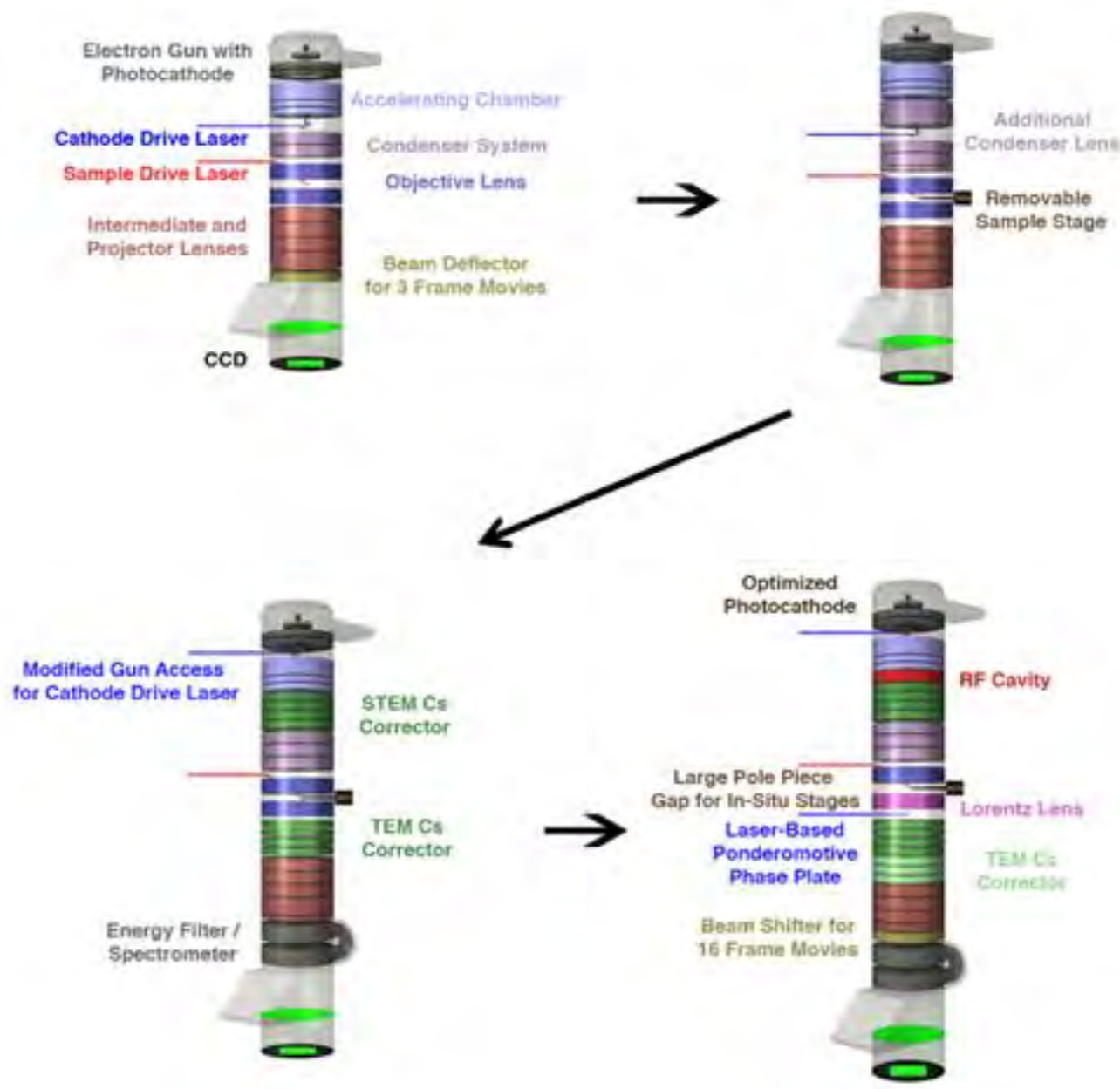

Figure 38. The evolution of single-shot DTEM columns from the earliest instrument to the proposed column for a UTEM.

\subsection{The Photo-emitter for UTEM}

Key to the development of a true (sub-nanosecond) ultrafast transmission electron microscope (UTEM) with sufficiently high spatial resolution for breakthrough scientific studies of single-shot (i.e., non-perturbative) events ${ }^{211}$ will be the brightness of the electron source generated by the laser-driven electron gun. Here there is a key difference to the physics that controls the emission. Specifically, the ultrafast regime is defined as using a laser pulse duration shorter than the timeof-flight in the photoelectron gun - which is typically 0.1-1ns. Under these conditions, the spatial (or transverse) emittance is given by

$$
\varepsilon_{T}=\frac{\hbar}{m c} \sqrt{\left\langle x^{2}\right\rangle\left\langle k_{x}^{2}\right\rangle}=\frac{p_{0}}{m c} r \Delta \theta
$$

where $r$ is the transverse size of the electron bunch, $\Delta \theta$ is its transverse divergence, $m$ is the electron mass, and $p_{0}$ is the momentum of the accelerated electron (i.e., $p_{0}=\sqrt{2 m q V_{D C}}$ in the non-relativistic limit with $q$ the electron charge and 
$V_{D C}$ the acceleration voltage). Because the transverse emittance is conserved in unperturbed electron pulse propagation, as in conventional geometric optics, the beam-size-divergence product, $\mathrm{r} \Delta \theta$, will dictate the "best" spatial resolution attainable in any ultrafast transmission electron microscope. In particular, the beam divergence, $\Delta \theta$, over the aberrationminimized entrance aperture size of the microscope's objective lens of focal length $f$ will determine the spatial resolution $\delta x$ through the simple relation, $\delta x=(f . \Delta \theta) / N_{\mathrm{p}}$, where $N_{\mathrm{p}} \times N_{\mathrm{p}}$ represents the number of pixels on the CCD image. Similarly, one can show that the post-specimen spatial separation of the first order diffraction spot from central undiffracted beam is proportional to (r. $\Delta \theta)^{-1}$, implying that image processing (such as phase-contrast imaging) and timeresolved diffraction experiments in a UTEM will only be possible with a high brightness electron source.

A seemingly straightforward method of reducing the transverse beam emittance is to decrease the electron source size $r$ by reducing the laser spot size on the electron gun's photocathode. However, if electron pulse break-up is to be avoided, ${ }^{212}$ this approach is limited by virtual cathode formation when the space-charge field between the electron pulse and the photocathode exceeds the DC gun field - the short-pulse Child's Law. For reasonable contrast (grey levels) in single-shot imaging, the Rose criterion ${ }^{209}$ requires $\sim 10^{8}$ electrons/pulse for a $1 \mathrm{k} \times 1 \mathrm{k} \mathrm{CCD}$, which, for typical acceleration field strengths of $10-20 \mathrm{kV} / \mathrm{cm}$, then implies a source size $\mathrm{r}>0.5 \mathrm{~mm}$. Moreover, the product $p_{0} \mathrm{r}$ is independent of the applied gun voltage $V_{D C}$ and only weakly dependent on the gun's acceleration distance. Consequently, any meaningful reduction in $\varepsilon_{\mathrm{T}}$ will likely only come from $\Delta \theta$; specifically, a reduction in the transverse momentum distribution of the electrons emitted from the photocathode.
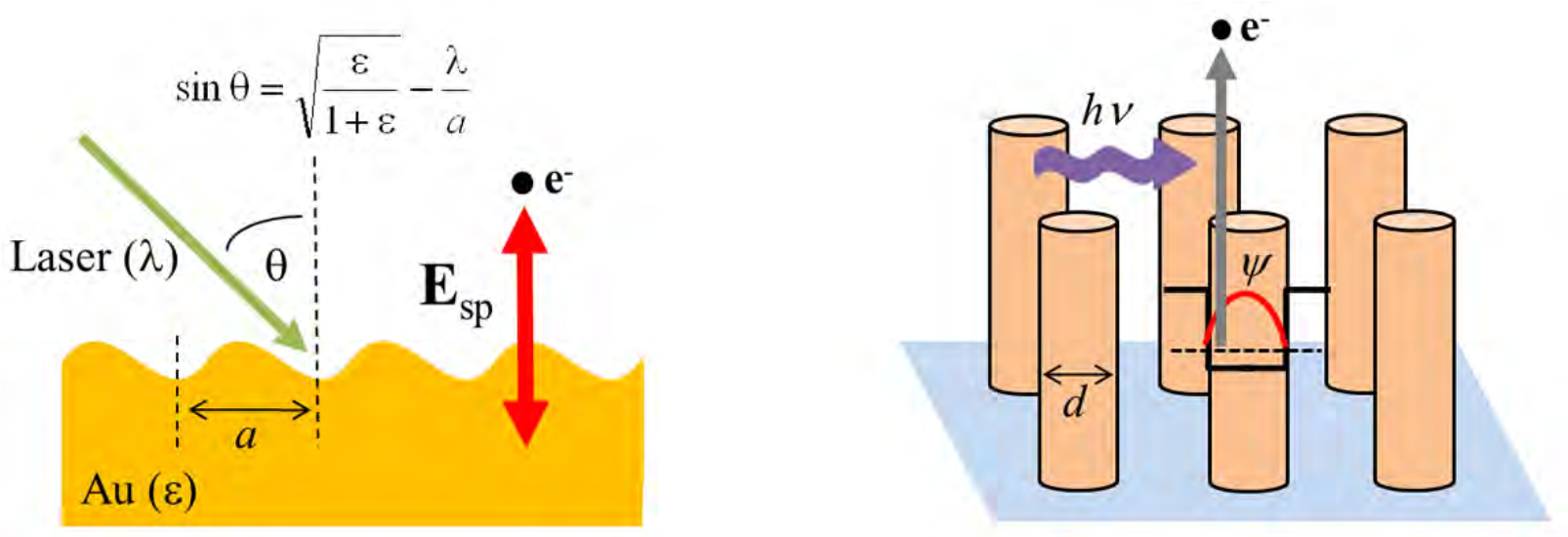

Figure 39. Schematic of the (a) Plasmon assisted and (b) nano patterned approach to increasing gun brightness.

There are arguably only two means of achieving this in a laser-driven electron gun (Figure 39): (i) employ the incident oscillating laser field to induce a response in the photocathode material that reduces $\Delta \theta$, or (ii) engineer the electronic structure of the photocathode material as $\Delta \theta$ is dependent upon the transverse momentum variance of the state from which the electrons originate in the photocathode. Plasmon-assisted photoemission ${ }^{213,214}$, an example of the former active method, is currently being investigated. ${ }^{215}$ End-on semiconductor (e.g., $\mathrm{GaSb}^{216}$ ) quantum wire emitters in an anodic alumina matrix ${ }^{217}$ (30-50nm pores in a $\sim 100 \mathrm{~nm}$ hexagonal two-dimensional lattice) provide an interesting nanostructured photocathode for the latter passive method. A goal of achieving an order of magnitude reduction in $\Delta \theta$ over that produced by conventional single-photon emission from a metal photocathode ${ }^{218}$ should allow a sub-ns UTEM to operate with a spatial resolution in the desired 1-10nm range.

Combining the equations for the emission of an ultrafast bunch of electrons with the equations for coherent flux for DTEM, we can derive an understanding of the operating parameters for a UTEM. For a 200kV UTEM, a laser-driven DC 
gun producing $N \approx 10^{8}$ electrons/pulse near the short-pulse Child's Law limit (defined as $\varepsilon_{0} E_{D C}=\frac{N e}{\pi r^{2}}$ ) may be expected to have a coherent fluence $N_{C}=\frac{\lambda^{2} N}{(r \alpha)^{2}} \approx 10^{-3}$ for electron generation by single-photon photoemission (with $\alpha$ $\approx 1 \mathrm{mrad}$ ) and typical DC field strengths of $10-20 \mathrm{kV} / \mathrm{cm}$. In the ultrafast regime (defined as the incident drive laser pulse duration less than the time-of-flight in the electron gun), this value for $N_{C}$ is of course independent of the electron pulse duration $\Delta t$. Using the definition of the coherence factor, $f_{\text {coherence }}=\lambda /(\delta x . \theta)$, where $\delta x$ is the size of the smallest feature to be resolved and $\theta$ is the half-width of the angular acceptance of the microscope's objective lens, the number of detected electrons per pixel may be found using equation (3). To satisfy the Rose criterion for reasonable contrast (grey levels) in single-shot imaging, $N$ should greater than about 100 , which for $\theta \approx 10 \mathrm{mrad}$ (e.g., $10 \mathrm{~mm}$ focal length with a $0.1 \mathrm{~mm}$ acceptance aperture) can only be achieved for feature sizes $\delta x>100 \mathrm{~nm}$. Thus, if space-charge effects are not significant and the electron pulse duration is in the 10-100ps range (a reasonable value for free-space propagation over $<1 \mathrm{~m}$ ), a STR of $\sim 10^{-17} \mathrm{~ms}$ can be expected. Not surprisingly, this performance is comparable to that of the LLNL DTEM, although the pulse duration is now much shorter. The above analysis provides two clear approaches to improving the performance of a UTEM. First, if the acceptance aperture $\theta$ of the objective lens is increased to around 30mrad, features as small as $30 \mathrm{~nm}$ may now be resolved. Second, if the initial divergence $\alpha$ of the electrons emitted from the gun's photocathode were reduced by an order of magnitude (e.g., through photocathode engineering or using a different emission mechanism), then $\delta x>10 \mathrm{~nm}$ may now be resolved. A combination of the two would allow feature sizes of a few nanometers to be observed, potentially pushing the UTEM's STR well below $10^{-18} \mathrm{~ms}$ (again assuming that stochastic blurring effects do not degrade this resolution).

\subsection{The Laser Drive System}

The design for a short pulse laser radiation source for the UTEM is based on standard modern laser technology. Due to the required shot-to-shot stability and pulse duration flexibility (from the sub-ps to nanosecond regimes), the laser system will employ diode-pumped solid-state laser technology in combination with chirped-pulse amplification (CPA). ${ }^{219}$ As shown in Figure 40, the proposed laser system has two main components; 1) a shot-noise-limited (RIN), 50MHz, diode-pumped fiber laser delivering $\sim 100 \mathrm{fs}$ pulses at around 1050nm and 2) a synchronized pulsed diode-pumped Nd:glass regenerative amplifier operating at $1-10 \mathrm{~Hz}^{220-223}$. The pulse stretcher and compressor in the CPA technique will allow pulse durations of $\sim 1 \mathrm{~ns}$ (uncompressed) to less than 1ps (fully compressed) to be generated, while operation of the regenerative amplifier as an unseeded, Q-switched and cavity-dumped laser resonator will provide for an output pulse duration of $\sim 10 \mathrm{~ns}$. The expected $\sim 10 \mathrm{~mJ}$ amplified pulse energies at $\lambda \approx 1050 \mathrm{~nm}$ will be sufficient for both the photo-generation of the required $\sim 10^{8}$ electrons/pulse (single-shot imaging with a $1 \mathrm{k} \times 1 \mathrm{k}$ CCD detection area) from the UTEM's $100-300 \mathrm{kV}$ electron gun and the synchronized specimen perturbation. Specifically, using standard harmonic generation techniques, the laser system will readily produce the $\sim 100 \mu \mathrm{J}$ pulse energies required in the ultraviolet (e.g., the fourth harmonic at $\sim 260 \mathrm{~nm}$ ) for the expected $10^{-6}-10^{-7}$ single-photon photoemission efficiency from both metal (e.g., $\mathrm{Ta}^{224}$ ) and semiconductor quantumwire based photocathodes. Moreover, the $\sim 530 \mathrm{~nm}$ green second harmonic wavelength is ideal for plasmon-assisted photoemission from gold. ${ }^{214}$

Figure 40 also shows two straightforward upgrades to the laser system to provide additional capabilities for the UTEM. First, the inclusion of a 1-2Hz diode-pumped Nd:glass power amplifier will allow for the generation of the 16, 10mJ sequential pulses required for the "movie-mode" of operation for the UTEM. Second, a multi-kHz, diode-pumped, fiber amplifier ${ }^{225}$ will allow perturbative studies on reversible phenomena to also be performed. In addition, the laser system design allows for a further upgrade, to provide broad wavelength tunability, for example, to target a particular specimen 
absorption band. In this case, a sub-ps continuum generated after pulse compression of the $\mathrm{kHz}$ fiber amplifier output ${ }^{226}$ will be passed through an optical parametric amplifier pumped by the green and ultra-violet harmonics of the high-power Nd:glass system.

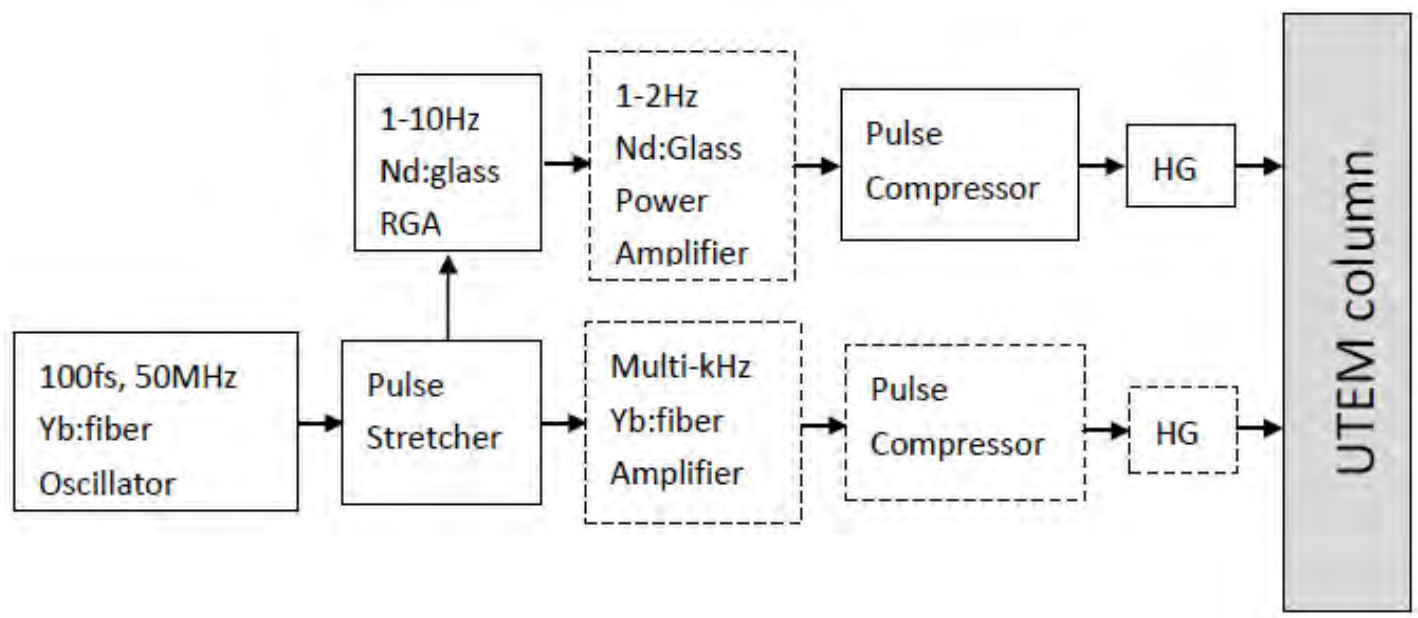

Figure 40. Schematic of the proposed laser system for the single-shot UTEM: Dashed boxes indicate position of components for two upgrades $(\mathrm{HG}=$ harmonic generation).

\subsection{Spectroscopy}

For the operation of a functional UTEM care must be taken to avoid or compensate for any deleterious space-charge effects. In the transverse direction (perpendicular to the beam propagation direction), global space-charge effects may be compensated for through the use of conventional magnetic lenses. In the longitudinal (temporal) pulse dimension, spacecharge effects can significantly temporally broaden the electron pulse and increase its energy spread $\Delta \mathrm{E}-$ the latter being particularly problematic for spatial focusing $\left(\mathrm{C}_{\mathrm{c}}\right.$ correction $)$ and energy resolution in EELS. Synchronized TM $\mathrm{T}_{010}-\mathrm{mode}_{\mathrm{RF}}$ cavities have been proposed ${ }^{207,227}$ and are now being used ${ }^{228}$ to compensate for, and indeed reverse, these effects. In the UTEM case, we also can employ a RF cavity (positioned in the column after the DC photo-gun), to reduce the energy spread $\Delta$ E. Specifically, simulations ${ }^{229}$ indicate that, after generation in the DC gun using a sub-ps laser pulse, a suitably phased RF cavity which simply stops the electron pulse's temporal expansion, but does not initiate its compression (i.e., simply nulls its longitudinal momentum chirp), can generate a pulse with an duration of $\sim 10 \mathrm{ps}$ and $\Delta \mathrm{E} \sim 0.01 \mathrm{eV}$. This mode of operation would still provide several orders of magnitude improvement in temporal resolution over current single-shot imaging dynamic TEMs while not requiring pre-specimen $\mathrm{C}_{\mathrm{c}}$ correction optics and, most importantly, for several of the scientific challenges, provide the capability to perform very high spectral resolution EELS.

\subsection{Potential UTEM Resolution}

We have now established basic electron optical parameters that allow us to estimate the potential imaging abilities of a UTEM. The brightness of the photocathode described above is sufficient to fulfill the Rose criterion if we assume a beam semi-convergence angle of $30 \mathrm{mrad}$. Pulse compression by a RF cavity will result in an energy width of $10 \mathrm{meV}$. However, for the following calculations, we will assume a more conservative value of $1 \mathrm{eV}$ to allow direct comparison with 
conventional systems. A wide gap objective lens suitable for in situ experiments is assumed with a gap of $9 \mathrm{~mm}$ between the pole pieces. This will allow all of the current in situ stages to be incorporated and provide space for the design of new stages. The coefficient of chromatic aberration for such a lens can be estimated to $2.4 \mathrm{~mm}$, including relativistic correction for $200 \mathrm{kV}$ electrons. $\mathrm{A} \mathrm{C}_{\mathrm{s}}$ corrector increases this value to $2.6 \mathrm{~mm}$. Contrast transfer function (CTF) calculations are based on Ishizuka's non-linear imaging theory ${ }^{230}$ with extensions for $\mathrm{C}_{\mathrm{c}}$ corrected systems. ${ }^{23}$ The green curve in Figure $41 \mathrm{a}$ shows the phase contrast transfer function (PCTF) of a standard TEM with Schottky emitter with a semi-convergence of $0.1 \mathrm{mrad}$. The damping envelop of spatial coherence is strongly influenced by a semi-convergence of $30 \mathrm{mrad}$ and is the major limiting parameter for contrast transfer. The damping envelope of temporal coherence does not limit the optical abilities (blue curve, Figure 41a). Under these circumstances, defocus-induced phase contrast (black, Figure 41a), as well as the amplitude contrast transfer function (ACTF) (red, Figure 41a) is close to zero for almost all spatial frequencies. Direct phase imaging using a phase plate results in contrast equivalent to the ACTF because phase information is shifted into the amplitude. This is preferable because, for thin samples, the phase of the exit surface wave is more sensitive to the sample than the amplitude. $\mathrm{C}_{\mathrm{s}}$ correction can mitigate spatial coherence damping this problem because $\mathrm{C}_{\mathrm{s}}$ balances the influence of beam convergence. ${ }^{23}$
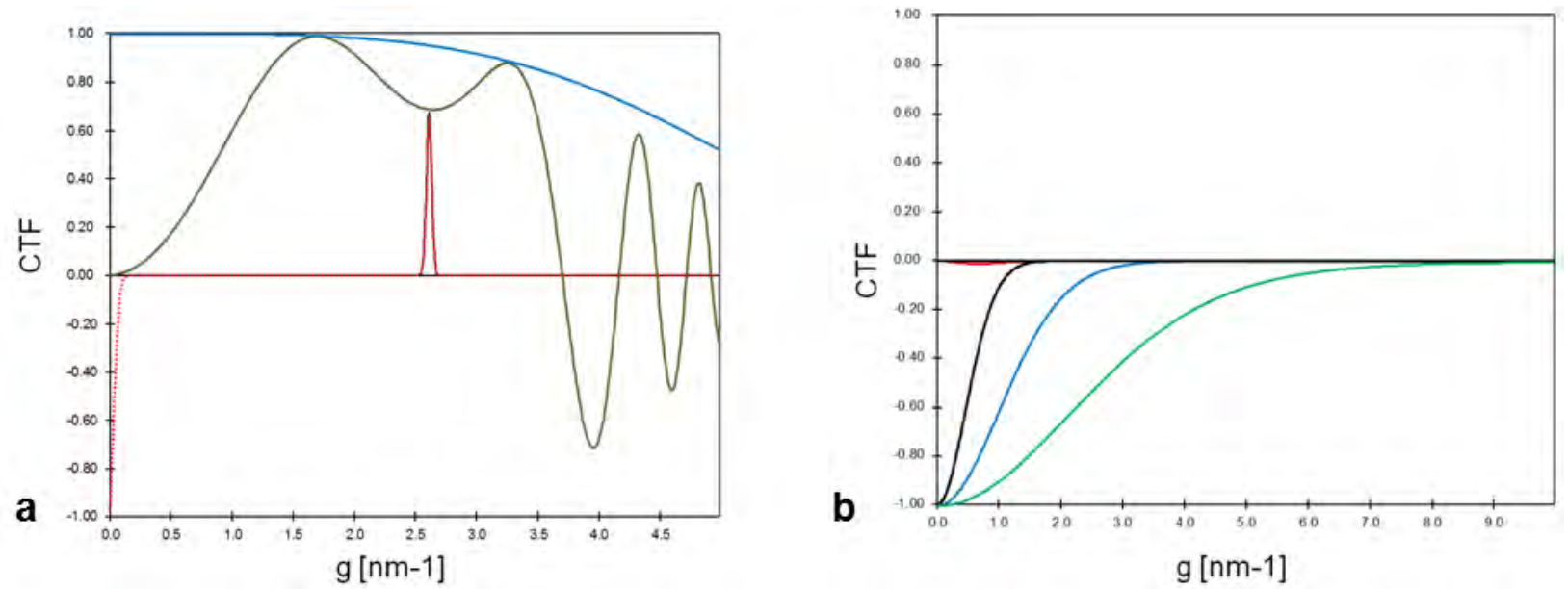

Figure 41. (a) Contrast transfer for an uncorrected TEM: green (standard Schottky FEG), red (ACTF), and black (PCTF): UTEM with $\alpha=30 \mathrm{mrad}$; blue: damping envelope of temporal coherence. (b) Contrast transfer for the spherically and chromatically corrected UTEM: red: $\mathrm{PCTF}$ for $\mathrm{C}_{5}=10 \mathrm{~mm}$; black: ACTF for $\mathrm{C}_{5}=10 \mathrm{~mm}$; blue: ACTF for $\mathrm{C}_{5}=1.0 \mathrm{~mm}$; green: $\mathrm{ACTF}$ for $\mathrm{C}_{5}=0.1 \mathrm{~mm}$ (note that these resolution limits assume that the space-charge effects caused by the lenses in the column do not degrade the resolution).

The high value of semi-convergence requires equally small values for all coefficients of spherical aberration up to the fifth order: $\mathrm{C}_{1}$ (defocus), $\mathrm{C}_{3}\left(\mathrm{C}_{\mathrm{s}}\right)$, and $\mathrm{C}_{5} . \mathrm{C}_{5}$ can be estimated to about $10 \mathrm{~mm}$ for the objective lens described here. Scherzer ${ }^{231}$ suggested a set of equations for optimum values of $\mathrm{C}_{1}$ and $\mathrm{C}_{3}$ for a fixed $\mathrm{C}_{5}$. Using this set of parameters results in the red (PCTF) and in the black curve (direct phase contrast or ACTF) shown in Figure 41b. The PCTF shows very low contrast transfer, which is typical for $\mathrm{C}_{\mathrm{s}}$ corrected instruments at low spatial frequencies. Defocusing cannot be used to improve phase contrast transfer as it will strongly increase damping from spatial coherence. Therefore, direct phase imaging or amplitude imaging are the only methods available to create high contrast transfer (black, blue, and green curves, Figure 41b). Resolution is, nevertheless, limited to $\sim 1 \mathrm{~nm}$ by the $\mathrm{C}_{5}$ value of the objective lens. However, atomic level resolution, can be achieved by $\mathrm{C}_{5}$ correction (blue and green curve, Figure $41 \mathrm{~b}$ ). A $\mathrm{C}_{5}$ value of $0.1 \mathrm{~mm}$ gives a contrast transfer up to $0.2 \mathrm{~nm}$ but requires an improvement in measurement accuracy for $\mathrm{C}_{5}$ and precise adjustment of $\mathrm{C}_{1}$ and $\mathrm{C}_{3}$. The first prototype of the TEAM $\mathrm{C}_{\mathrm{c}}$ corrector $^{19}$ is capable of a precision of $+/-1 \mathrm{~mm}$ for $\mathrm{C}_{5}$ (blue curve, Figure $41 \mathrm{~b}$ ), resulting in a 
resolution of about $0.5 \mathrm{~nm}$. Improvement of measurement accuracy by an order of magnitude appears feasible, which is required for a resolution of $0.2 \mathrm{~nm}$ (green curve, Figure 41b). Using such high convergence angles has a consequence for the depth of focus in the image, which makes alignment a little more complicated. However, it does have the added benefit of permitting three-dimensional imaging. ${ }^{232,233}$ It also should be noted that the high convergence angles can be reduced for imaging with nanosecond pulses.

\subsection{The Significance of Single-shot UTEM in the Global Research Community}

The coupling of ultrafast temporal resolution with the spatial resolution of the transmission electron microscope is a goal that is currently growing across the world. In addition to the previously described work of Bostanjoglo, ${ }^{28,197,198}$ Zewail and co-workers, ${ }^{172,185-187,234}$ the LLNL DTEM project, ${ }^{29,30,193-196}$ and the work that is currently underway at UC-Davis (which is being transferred to PNNL in 2012), there are projects that are building new microscopes in Japan, Switzerland, Germany and Russia. There is also a large-scale project for the development of DTEM being initiated in Canada. While these projects have different goals in terms of the science that they aim to address, they are all linked by similarities in the design of the instrumentation - they all use lasers coupled to a photoemission gun to achieve temporal resolution. While the number of groups working in this area is still small, the number of projects being initiated is growing quickly. The reason for this growth is a building realization that the technology for the ultrafast microscope is within reach and can provide unique and complementary information to synchrotron- and optical-based techniques. The community for ultrafast TEM is coming together to work toward a common platform, making it an opportune time to define the future for this method. Such capabilities can make significant impacts on the Grand Challenges in materials under extreme conditions and energy technologies defined in recent DOE workshop reports.

\subsection{Timeline for Technology Development}

The goal in the development of a UTEM is to use the advanced electron optics that have come from recent developments in the TEAM project (Cs and Cc correction) and through extensive work in the cryo-electron microscopy community (phase plates). Coupled to this column design will be technologies originating from LLNL's DTEM project (movie mode) and UC-Davis' DTEM (gun design for photoemission). The only truly new technology that will need to be added to the column will be the addition of an RF cavity to act as a temporal lens. Given that these components have all been successfully implemented previously, the first stage of the project to install and align the microscope column is expected to take about 2 years from the placement of the order. A development column for acceleration voltages of 80 to $200 \mathrm{kV} / 300 \mathrm{kV}$ with high electrical and mechanical stability will be at the core of the project. This instrument has to be optimized for in situ TEM, which requires a wide gap between the objective pole pieces of at least $10 \mathrm{~mm}$ and spherical and chromatic aberration correction for imaging mode. Spherical aberration correction is necessary to compensate the large beam convergence of the electron emitter system, while chromatic correction allows use of a wide gap objective lens, and to improve resolution for samples in liquid or gaseous environment. For sub-nanosecond time scales the distance between electron emitter and sample must be kept short, and crossovers in the condenser system have to be minimized to maintain temporal resolution of the electron beam. This system is then used to integrate, in successive steps, the laser for triggering events on the sample and creating electron pulses, a detection system optimized for single shot and movie mode, a photoemission gun (UC Davis concept), and a RF cavity to improve time and energy resolution. Further components are phase plates that will enhance contrast transfer significantly (see Figure 41) and sample stages for specific in situ experiments. The final stage of this project is to implement a patterned photoemission gun with increased brightness that will lead to a truly ultrafast single shot microscope (a full timeline is shown in Table 1). This concept ensures that scientific experiments can be performed in the project's early stages. The instrument concept is unique from the beginning (wide gap objective lens with $\mathrm{C}_{\mathrm{c}}$ correction) and will add further unique abilities throughout the project. 
Given that the electron optics will be modified with the $\mathrm{C}_{\mathrm{c}}$ corrector and the phase plate, it is anticipated that this first gun will produce an improvement in performance over the existing UC-Davis instrument. It also should be noted here that further improvements in spatiotemporal resolution may be possible with further emitter improvements, and these improvements will be retrofittable to this microscope.

Table 1. Timeline for the construction of a UTEM.

\begin{tabular}{|c|c|c|c|c|c|c|}
\hline & year 1 & year 2 & year 3 & year 4 & year 5 & year 6 \\
\hline \multirow[t]{8}{*}{ Stage I } & $\begin{array}{l}\text { Design concept and } \\
\text { design study }\end{array}$ & & & & & \\
\hline & $\begin{array}{l}\text { design study: source, } \\
\text { electron optical system } \\
\text { stage II }\end{array}$ & & & & & \\
\hline & microscope column & & & & & \\
\hline & $\begin{array}{l}\text { Installation and test, } \\
\text { set up of the stage for } \\
\text { planned in-situ } \\
\text { experiments, first } \\
\text { experiments }\end{array}$ & & & & & \\
\hline & $\begin{array}{l}\text { nanosecond laser } \\
\text { system: construction }\end{array}$ & & & & & \\
\hline & & $\begin{array}{l}\text { Modify gun for ns pulse, } \\
\text { develop aberration } \\
\text { correction }(\mathrm{Cs} \text { and } \mathrm{Cc}) \text { in } \\
\text { pulsed mode }\end{array}$ & $\begin{array}{l}\text { improve } \\
\text { measurement } \\
\text { accuracy of } \\
\text { aberration } \\
\text { coefficients }\end{array}$ & & & \\
\hline & stage development & & stage development & & & \\
\hline & & Fast detection system & $\begin{array}{l}\text { Fast detection } \\
\text { system: movie } \\
\text { mode }\end{array}$ & & & \\
\hline \multirow[t]{6}{*}{ Stage II } & & & $\begin{array}{l}\text { Ultrafast gun: } \\
\text { construction }\end{array}$ & & & \\
\hline & & & & $\begin{array}{l}\text { construct RF } \\
\text { cavity puls } \\
\text { compression } \\
\text { system }\end{array}$ & & \\
\hline & & & & $\begin{array}{l}\text { Picosecond laser } \\
\text { system: } \\
\text { construction }\end{array}$ & & \\
\hline & & & & $\begin{array}{l}\text { Integration and } \\
\text { test: gun, RF } \\
\text { cavity, laser, } \\
\text { detector }\end{array}$ & & \\
\hline & & & & & $\begin{array}{l}\text { Shake down: } \\
\text { tests and first } \\
\text { experiments }\end{array}$ & \\
\hline & & & & & & $\begin{array}{l}\text { Facility } \\
\text { operation }\end{array}$ \\
\hline
\end{tabular}




\section{References}

$1 \quad$ Advanced Research Instrumentation and Facilities. (National Academy of Sciences, 2006).

2 DOE_Workshop. New Frontiers in Characterizing Biological Systems. (Department of Energy, 2009), $<$ http://genomicscience.energy.gov/characterization/> (2009).

3 Biosystems Design Workshop., (Biological and Environmental Research US Department of Energy, 2012), 2012).

4 Complex Systems Science for Subsurface Fate and Transport. (Biological and Environmental Research US Department of Energy, 2009),

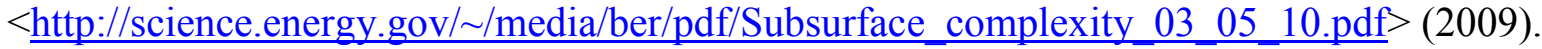

5 DOE Workshops. Opportunities for Catalysis in the 21st Century; Basic Research Needs for Carbon Capture: Beyond 2020; Report of the Basic Energy Sciences Workshop on Compact Light Sources; Future Science Needs and Opportunities for Electron Scattering: Next - Generation Instrumentation and Beyond; Nanoscience Research for Energy Needs, < http://science.energy.gov/bes/news-andresources/reports/workshop-reports/>

6 DOE_workshop. Nanoscience Research for Energy Needs. (Department of Energy, 2005), $<$ http://science.energy.gov/ /media/bes/pdf/reports/files/nren_rpt.pdf $>$ (2005).

7 Wayne E. King, G. H. C., Alan Frank, Bryan Reed, John F. Schmerge, Bradley J. Siwick, Brent C. Stuart and Peter M. Weber. Ultrafast electron microscopy in materials science, Biology, and chemistry. Journal of Applied Physics 97 (2005).

8 Stach, E. Real-time observations with electron microscopy. Materials Today 11, Supplement, 50-58, doi:10.1016/s1369-7021(09)70007-0 (2008).

9 Browning, N. D., Campbell, G. H., Evans, J. E., LaGrange, T. B. \& Reed, B. W. Electron Microscopy and Spectroscopy on the Ultrafast Timescale. ChemPhysChem 11, 781-782, doi:10.1002/cphc.200900937 (2010).

10 Barwick, B., Park, H. S., Kwon, O.-H., Baskin, J. S. \& Zewail, A. H. 4D Imaging of Transient Structures and Morphologies in Ultrafast Electron Microscopy. Science 322, 1227-1231, doi:10.1126/science.1164000 (2008).

11 Haider, M., Uhlemann, S., Schwan, E., Rose, H., Kabius, B. \& Urban, K. Electron microscopy image enhanced. Nature 392, 768-769 (1998).

12 Kabius, B., Haider, M., Uhlemann, S., Schwan, E., Urban, K. \& Rose, H. First application of a spherical-aberration corrected transmission electron microscope in materials science. Journal of Electron Microscopy 51, S51-S58, doi:10.1093/jmicro/51.Supplement.S51 (2002).

13 Mantl, S., Zhao, Q. T., and Kabius, B. Self-assembled patterning of ultrathin silicides by local oxidation. MRS Bulletin 24, 31-35 (1999). 
14 Lentzen, M., Jahnen, B., Jia, C. L., Thust, A., Tillmann, K. \& Urban, K. High-resolution imaging with an aberration-corrected transmission electron microscope. Ultramicroscopy 92, 233-242, doi:10.1016/s0304-3991(02)00139-0 (2002).

15 Dellby, N., Krivanek, L., Nellist, D., Batson, E. \& Lupini, R. Progress in aberration-corrected scanning transmission electron microscopy. Journal of Electron Microscopy 50, 177-185, doi:10.1093/jmicro/50.3.177 (2001).

16 van Benthem, K., Lupini, A. R., Oxley, M. P., Findlay, S. D., Allen, L. J. \& Pennycook, S. J. Threedimensional ADF imaging of individual atoms by through-focal series scanning transmission electron microscopy. Ultramicroscopy 106, 1062-1068, doi:10.1016/j.ultramic.2006.04.020 (2006).

17 Houdellier, F., Warot-Fonrose, B., Hÿ, M. J., CEMES-CNRS, n. \& France, n. New Electron Energy Loss Magnetic Chiral Dichroïsm (EMCD) configuration using an aberration-corrected transmission electron microscope. Microscopy and Microanalysis 13, 48-49, doi:doi:10.1017/S1431927607080245 (2007).

18 Kirkland, A., Chang, L.-Y., Haigh, S. \& Hetherington, C. Transmission electron microscopy without aberrations: Applications to materials science. Current Applied Physics 8, 425-428, doi:10.1016/j.cap.2007.10.065 (2008).

19 Kabius, B., Hartel, P., Haider, M., Müller, H., Uhlemann, S., Loebau, U., Zach, J. \& Rose, H. First application of Cc-corrected imaging for high-resolution and energy-filtered TEM. Journal of Electron Microscopy 58, 147-155, doi:10.1093/jmicro/dfp021 (2009).

20 Berger, A., Mayer, J. \& Kohl, H. Detection limits in elemental distribution images produced by energy filtering TEM: case study of grain boundaries in Si3N4. Ultramicroscopy 55, 101-112, doi:10.1016/0304-3991(94)90084-1 (1994).

21 Zuo, J. M., Kim, M., O'Keeffe, M. \& Spence, J. C. H. Direct observation of d-orbital holes and Cu-Cu bonding in Cu2O. Nature 401, 49-52 (1999).

22 Robertson, I. M., Ferreira, P. J., Dehm, G., Hull, R. \& Stach, E. A. Visualizing the Behavior of Dislocations-Seeing is Believing. MRS Bulletin 33, 122-131, doi:doi:10.1557/mrs2008.25 (2008).

23 Kabius, B. \& Rose, H. in Advances in Imaging and Electron Physics Vol. Volume 153 (ed W. Hawkes Peter) 261-281 (Elsevier, 2008).

24 Evans, J. E., Jungjohann, K. L., Browning, N. D. \& Arslan, I. Controlled Growth of Nanoparticles from Solution with In Situ Liquid Transmission Electron Microscopy. Nano Letters 11, 2809-2813, doi:10.1021/nl201166k (2011).

25 Zhong, L., Matthews, J. F., Hansen, P. I., Crowley, M. F., Cleary, J. M., Walker, R. C., Nimlos, M. R., Brooks Iii, C. L., Adney, W. S., Himmel, M. E. \& Brady, J. W. Computational simulations of the Trichoderma reesei cellobiohydrolase I acting on microcrystalline cellulose I $\beta$ : the enzyme-substrate complex. Carbohydrate Research 344, 1984-1992, doi:10.1016/j.carres.2009.07.005 (2009).

26 Merkle, K. L., Thompson, L. J. \& Philipp, F. In-Situ HREM Studies of Grain Boundary Migration. Interface Science 12, 277-292 (2004).

27 Battaglia, M., Contarato, D., Denes, P., Doering, D., Giubilato, P., Kim, T. S., Mattiazzo, S., Radmilovic, V. \& Zalusky, S. A rad-hard CMOS active pixel sensor for electron microscopy. Nuclear Instruments and Methods in Physics Research Section A: Accelerators, Spectrometers, Detectors and Associated Equipment 598, 642-649, doi:10.1016/j.nima.2008.09.029 (2009). 
28 Bostanjoglo, O. \& Leidtke, R. Advances in Imaging and Electron Physics 60, 451 (1980).

29 Reed, B. W., Armstrong, M. R., Browning, N. D., Campbell, G. H., Evans, J. E., LaGrange, T. \& Masiel, D. J. The Evolution of Ultrafast Electron Microscope Instrumentation. Microscopy and Microanalysis 15, 272-281, doi:doi:10.1017/S1431927609090394 (2009).

30 Kim, J. S., LaGrange, T., Reed, B. W., Taheri, M. L., Armstrong, M. R., King, W. E., Browning, N. D. \& Campbell, G. H. Imaging of Transient Structures Using Nanosecond in Situ TEM. Science 321, 14721475, doi:10.1126/science.1161517 (2008).

31 Emma P, Akre R, Arthur J, Bionta R, Bostedt C, Bozek J, Brachmann A, Bucksbaum P, Coffee R, Decker, F. J., Ding Y, Dowell D, Edstrom S, Fisher A, Frisch J, Gilevich S, Hastings J, Hays G, Hering $\mathrm{Ph}$, Huang Z, Iverson R, Loos H, Messerschmidt M, Miahnahri A, Moeller S, Nuhn, H. D., Pile G, Ratner D, Rzepiela J, Schultz D, Smith T, Stefan P, Tompkins H, Turner J, Welch J, White W, Wu J, Yocky G \& Galayda J. First lasing and operation of an angstrom-wavelength free-electron laser. Nat Photon 4, 641-647 (2010).

32 Chapman, H. N., Fromme, P., Barty, A., White, T. A., Kirian, R. A., Aquila, A., Hunter, M. S., Schulz, J., DePonte, D. P., Weierstall, U., Doak, R. B., Maia, F. R. N. C., Martin, A. V., Schlichting, I., Lomb, L., Coppola, N., Shoeman, R. L., Epp, S. W., Hartmann, R., Rolles, D., Rudenko, A., Foucar, L., Kimmel, N., Weidenspointner, G., Holl, P., Liang, M., Barthelmess, M., Caleman, C., Boutet, S., Bogan, M. J., Krzywinski, J., Bostedt, C., Bajt, S., Gumprecht, L., Rudek, B., Erk, B., Schmidt, C., Homke, A., Reich, C., Pietschner, D., Struder, L., Hauser, G., Gorke, H., Ullrich, J., Herrmann, S., Schaller, G., Schopper, F., Soltau, H., Kuhnel, K.-U., Messerschmidt, M., Bozek, J. D., Hau-Riege, S. P., Frank, M., Hampton, C. Y., Sierra, R. G., Starodub, D., Williams, G. J., Hajdu, J., Timneanu, N., Seibert, M. M., Andreasson, J., Rocker, A., Jonsson, O., Svenda, M., Stern, S., Nass, K., Andritschke, R., Schroter, C.D., Krasniqi, F., Bott, M., Schmidt, K. E., Wang, X., Grotjohann, I., Holton, J. M., Barends, T. R. M., Neutze, R., Marchesini, S., Fromme, R., Schorb, S., Rupp, D., Adolph, M., Gorkhover, T., Andersson, I., Hirsemann, H., Potdevin, G., Graafsma, H., Nilsson, B. \& Spence, J. C. H. Femtosecond X-ray protein nanocrystallography. Nature 470, 73-77, doi:http://www.nature.com/nature/journal/v470/n7332/abs/10.1038-nature09750unlocked.html\#supplementary-information (2011).

33 Seibert, M. M., Ekeberg, T., Maia, F. R. N. C., Svenda, M., Andreasson, J., Jonsson, O., Odic, D., Iwan, B., Rocker, A., Westphal, D., Hantke, M., DePonte, D. P., Barty, A., Schulz, J., Gumprecht, L., Coppola, N., Aquila, A., Liang, M., White, T. A., Martin, A., Caleman, C., Stern, S., Abergel, C., Seltzer, V., Claverie, J.-M., Bostedt, C., Bozek, J. D., Boutet, S., Miahnahri, A. A., Messerschmidt, M., Krzywinski, J., Williams, G., Hodgson, K. O., Bogan, M. J., Hampton, C. Y., Sierra, R. G., Starodub, D., Andersson, I., Bajt, S., Barthelmess, M., Spence, J. C. H., Fromme, P., Weierstall, U., Kirian, R., Hunter, M., Doak, R. B., Marchesini, S., Hau-Riege, S. P., Frank, M., Shoeman, R. L., Lomb, L., Epp, S. W., Hartmann, R., Rolles, D., Rudenko, A., Schmidt, C., Foucar, L., Kimmel, N., Holl, P., Rudek, B., Erk, B., Homke, A., Reich, C., Pietschner, D., Weidenspointner, G., Struder, L., Hauser, G., Gorke, H., Ullrich, J., Schlichting, I., Herrmann, S., Schaller, G., Schopper, F., Soltau, H., Kuhnel, K.-U., Andritschke, R., Schroter, C.-D., Krasniqi, F., Bott, M., Schorb, S., Rupp, D., Adolph, M., Gorkhover, T., Hirsemann, H., Potdevin, G., Graafsma, H., Nilsson, B., Chapman, H. N. \& Hajdu, J. Single mimivirus particles intercepted and imaged with an X-ray laser. Nature 470, 78-81 (2011).

34 Henderson, R. The potential and limitations of neutrons, electrons and X-rays for atomic resolution microscopy of unstained biological molecules. Quarterly Reviews of Biophysics 28, 171-193, doi:doi:10.1017/S003358350000305X (1995). 
35 Hubbell, J. H., Gimm, H. A. \& Overbo, I. Pair, Triplet, and Total Atomic Cross Sections (and Mass Attenuation Coefficients) for $1 \mathrm{MeV}-100 \mathrm{GeV}$ Photons in Elements $\mathrm{Z}=1$ to 100. Journal of Physical and Chemical Reference Data 9, 1023-1148 (1980).

36 Chapman, H. N., Hau-Riege, S. P., Bogan, M. J., Bajt, S., Barty, A., Boutet, S., Marchesini, S., Frank, M., Woods, B. W., Benner, W. H., London, R. A., Rohner, U., Szoke, A., Spiller, E., Moller, T., Bostedt, C., Shapiro, D. A., Kuhlmann, M., Treusch, R., Plonjes, E., Burmeister, F., Bergh, M., Caleman, C., Huldt, G., Seibert, M. M. \& Hajdu, J. Femtosecond time-delay X-ray holography. Nature 448, 676-679 (2007).

37 Hau-Riege, S. P., London, R. A., Huldt, G. \& Chapman, H. N. Pulse requirements for x-ray diffraction imaging of single biological molecules. Physical Review E 71, 061919 (2005).

38 Siwick, B. J., Dwyer, J. R., Jordan, R. E. \& Miller, R. J. D. An Atomic-Level View of Melting Using Femtosecond Electron Diffraction. Science 302, 1382-1385, doi:10.1126/science.1090052 (2003).

39 Future Science Needs and Opportunities for Electron Scattering: Next-Generation Instrumentation and Beyond. 55 (2007), <http://www.sc.doe.gov/bes/reports/list.html.> (2007).

40 Patterson, G., Davidson, M., Manley, S. \& Lippincott-Schwartz, J. Superresolution Imaging using Single-Molecule Localization. Annual Review of Physical Chemistry 61, 345-367, doi:doi:10.1146/annurev.physchem.012809.103444 (2010).

41 Shi, L., Belchik, S. M., Wang, Z. M., Kennedy, D. W., Dohnalkova, A. C., Marshall, M. J., Zachara, J. M. \& Fredrickson, J. K. Identification and Characterization of UndA(HRCR-6), an Outer Membrane Endecaheme c-Type Cytochrome of Shewanella sp Strain HRCR-6. Appl. Environ. Microbiol. 77, 55215523, doi:10.1128/aem.00614-11 (2011).

42 Dohnalkova, A. C., Marshall, M. J., Arey, B. W., Williams, K. H., Buck, E. C. \& Fredrickson, J. K. Imaging Hydrated Microbial Extracellular Polymers: Comparative Analysis by Electron Microscopy. Appl. Environ. Microbiol. 77, 1254-1262, doi:10.1128/aem.02001-10 (2011).

43 Reda, T., Plugge, C. M., Abram, N. J. \& Hirst, J. Reversible interconversion of carbon dioxide and formate by an electroactive enzyme. Proceedings of the National Academy of Sciences of the United States of America 105, 10654-10658, doi:0801290105 [pii]10.1073/pnas.0801290105 (2008).

44 Galan, B. R., Schoffel, J., Linehan, J. C., Seu, C., Appel, A. M., Roberts, J. A., Helm, M. L., Kilgore, U. J., Yang, J. Y., DuBois, D. L. \& Kubiak, C. P. Electrocatalytic oxidation of formate by $\left[\mathrm{Ni}\left(\mathrm{P}(\mathrm{R}) 2 \mathrm{~N}\left(\mathrm{R}^{\prime}\right) 2\right) 2(\mathrm{CH} 3 \mathrm{CN})\right] 2+$ complexes. Journal of the American Chemical Society 133, $12767-$ 12779, doi:10.1021/ja204489e (2011).

45 Axley, M. J., Grahame, D. A. \& Stadtman, T. C. Escherichia coli formate-hydrogen lyase. Purification and properties of the selenium-dependent formate dehydrogenase component. J Biol Chem 265, 18213 18218 (1990).

46 Boyington, J. C., Gladyshev, V. N., Khangulov, S. V., Stadtman, T. C. \& Sun, P. D. Crystal structure of formate dehydrogenase $\mathrm{H}$ : catalysis involving Mo, molybdopterin, selenocysteine, and an Fe4S4 cluster. Science (New York, N.Y 275, 1305-1308 (1997).

47 Raaijmakers, H. C. \& Romao, M. J. Formate-reduced E. coli formate dehydrogenase H: The reinterpretation of the crystal structure suggests a new reaction mechanism. J Biol Inorg Chem 11, 849854, doi:10.1007/s00775-006-0129-2 (2006). 
48 Arner, E. S. Selenoproteins-What unique properties can arise with selenocysteine in place of cysteine? Exp Cell Res 316, 1296-1303, doi:S0014-4827(10)00094-7 [pii]10.1016/j.yexcr.2010.02.032 (2010).

49 Jacob, C., Giles, G. I., Giles, N. M. \& Sies, H. Sulfur and selenium: the role of oxidation state in protein structure and function. Angewandte Chemie (International ed 42, 4742-4758, doi:10.1002/anie.200300573 (2003).

50 Jacob, C., Knight, I. \& Winyard, P. G. Aspects of the biological redox chemistry of cysteine: from simple redox responses to sophisticated signalling pathways. Biological chemistry 387, 1385-1397 (2006).

51 Mota, C. S., Rivas, M. G., Brondino, C. D., Moura, I., Moura, J. J., Gonzalez, P. J. \& Cerqueira, N. M. The mechanism of formate oxidation by metal-dependent formate dehydrogenases. J Biol Inorg Chem 16, 1255-1268, doi:10.1007/s00775-011-0813-8 (2011).

52 Heider, J. \& Bock, A. Selenium metabolism in micro-organisms. Adv Microb Physiol 35, 71-109 (1993).

53 Givens, R. S., Rubina, M. \& Wirz, J. Applications of p-hydroxyphenacyl (pHP) and coumarin-4ylmethyl photoremovable protecting groups. Photochem. Photobiol. Sci. 11, 472-488, doi:10.1039/c2pp05399c (2012).

54 Bush, M., Ghosh, T., Tucker, N., Zhang, X. \& Dixon, R. Transcriptional regulation by the dedicated nitric oxide sensor, NorR: a route towards NO detoxification. Biochemical Society Transactions 39, 289293 (2011).

55 Sharina, I. G., Cote, G. J., Martin, E., Doursout, M. F. \& Murad, F. RNA splicing in regulation of nitric oxide receptor soluble guanylyl cyclase. Nitric Oxide 25, 265-274 (2011).

56 Price, M. S., Chao, L. Y. \& Marletta, M. A. Shewanella oneidensis MR-1 H-NOX Regulation of a Histidine Kinase by Nitric Oxide†. Biochemistry 46, 13677-13683, doi:10.1021/bi7019035 (2007).

57 Cruz-Garcia, C., Murray, A., Rodrigues, J., Gralnick, J., McCue, L., Romine, M., Loffler, F. \& Tiedje, J. Fnr (EtrA) acts as a fine-tuning regulator of anaerobic metabolism in Shewanella oneidensis MR-1. BMC Microbiology 11, 64 (2011).

58 Verma, S., Xiong, Y., Mayer, M. U. \& Squier, T. C. Remodeling of the Bacterial RNA Polymerase Supramolecular Complex in Response to Environmental Conditions $\dagger$. Biochemistry 46, 3023-3035, doi:10.1021/bi0621157 (2007).

59 Campbell, E. A., Greenwell, R., Anthony, J. R., Wang, S., Lim, L., Das, K., Sofia, H. J., Donohue, T. J. \& Darst, S. A. A Conserved Structural Module Regulates Transcriptional Responses to Diverse Stress Signals in Bacteria. Molecular cell 27, 793-805 (2007).

60 Plotkin, J. B. Cell biology. The lives of proteins. Science 331, 683-684, doi:331/6018/683 [pii] 10.1126/science.1202010 (2011).

61 Xiong, Y., Chen, B., Shi, L., Fredrickson, J. K., Bigelow, D. J. \& Squier, T. C. Targeted Protein Degradation of Outer Membrane Decaheme Cytochrome MtrC Metal Reductase in Shewanella oneidensis MR-1 Measured Using Biarsenical Probe CrAsH-EDT2. Biochemistry 50, 9738-9751, doi:10.1021/bi200602f(2011).

62 Plotkin, J. B. The Lives of Proteins. Science 331, 683-684, doi:10.1126/science.1202010 (2011).

63 Bigelow, D. J. \& Squier, T. C. Thioredoxin-dependent redox regulation of cellular signaling and stress response through reversible oxidation of methionines. Molecular BioSystems 7, 2101-2109 (2011). 
64 Weerapana, E., Wang, C., Simon, G. M., Richter, F., Khare, S., Dillon, M. B. D., Bachovchin, D. A., Mowen, K., Baker, D. \& Cravatt, B. F. Quantitative reactivity profiling predicts functional cysteines in proteomes. Nature 468, 790-795, doi:http://www.nature.com/nature/journal/v468/n7325/abs/nature09472.html\#supplementaryinformation (2010).

65 Steunou, A.-S., Jensen, S. I., Brecht, E., Becraft, E. D., Bateson, M. M., Kilian, O., Bhaya, D., Ward, D. M., Peters, J. W., Grossman, A. R. \& M., K. Regulation of nif gene expression and the energetics of N2 fixation over the diel cycle in a hot spring microbial mat. ISME Journal 2, 364-378 (2008).

66 Anthony, J. R., Warczak, K. L. \& Donohue, T. J. A transcriptional response to singlet oxygen, a toxic byproduct of photosynthesis. Proceedings of the National Academy of Sciences of the United States of America 102, 6502-6507 (2005).

67 Campbell, E. A., Greenwell, R., Anthony, J. R., Wang, S., Lim, L., Das, K., Sofia, H. J., Donohue, T. J. \& Darst, S. A. A conserved structural module regulates transcriptional responses to diverse stress signals in bacteria. Molecular cell 27, 793-805 (2007).

68 Uljana Mayer, M., Shi, L. \& Squier, T. C. One-step, non-denaturing isolation of an RNA polymerase enzyme complex using an improved multi-use affinity probe resin. Molecular bioSystems 1, 53-56 (2005).

69 Verma, S., Xiong, Y., Mayer, M. U. \& Squier, T. C. Remodeling of the bacterial RNA polymerase supramolecular complex in response to environmental conditions. Biochemistry 46, 3023-3035 (2007).

70 McCarthy, J. F. \& Zachara, J. M. Subsurface transport of contaminants. Environmental Science \& Technology 23, 496-502, doi:10.1021/es00063a001 (1989).

71 Clarke, T. A., Edwards, M. J., Gates, A. J., Hall, A., White, G. F., Bradley, J., Reardon, C. L., Shi, L., Beliaev, A. S., Marshall, M. J., Wang, Z., Watmough, N. J., Fredrickson, J. K., Zachara, J. M., Butt, J. N. \& Richardson, D. J. Structure of a bacterial cell surface decaheme electron conduit. Proceedings of the National Academy of Sciences 108, 9384-9389, doi:10.1073/pnas.1017200108 (2011).

72 Johs, A., Shi, L., Droubay, T., Ankner, J. F. \& Liang, L. Characterization of the Decaheme c-Type Cytochrome OmcA in Solution and on Hematite Surfaces by Small Angle X-Ray Scattering and Neutron Reflectometry. Biophys. J. 98, 3035-3043, doi:10.1016/j.bpj.2010.03.049 (2010).

73 Banfield, J. F., Welch, S. A., Zhang, H. Z., Ebert, T. T. \& Penn, R. L. Aggregation-based crystal growth and microstructure development in natural iron oxyhydroxide biomineralization products. Science 289, 751-754, doi:10.1126/science.289.5480.751 (2000).

74 Shi, L., Belchik, S. M., Plymale, A. E., Heald, S., Dohnalkova, A. C., Sybirna, K., Bottin, H., Squier, T. C., Zachara, J. M. \& Fredrickson, J. K. Purification and Characterization of the [NiFe]-Hydrogenase of Shewanella oneidensis MR-1. Appl. Environ. Microbiol. 77, 5584-5590, doi:10.1128/aem.00260-11 (2011).

75 Sharp, J. O., Schofield, E. J., Veeramani, H., Suvorova, E. I., Kennedy, D. W., Marshall, M. J., Mehta, A., Bargar, J. R. \& Bernier-Latmani, R. Structural Similarities between Biogenic Uraninites Produced by Phylogenetically and Metabolically Diverse Bacteria. Environmental Science \& Technology 43, 8295-8301, doi:10.1021/es901281e (2009). 
76 MacLean, L. C. W., Tyliszczak, T., Gilbert, P., Zhou, D., Pray, T. J., Onstott, T. C. \& Southam, G. A high-resolution chemical and structural study of framboidal pyrite formed within a low-temperature bacterial biofilm. Geobiology 6, 471-480, doi:10.1111/j.1472-4669.2008.00174.x (2008).

77 Molina, L. M., Lee, S., Sell, K., Barcaro, G., Fortunelli, A., Lee, B., Seifert, S., Winans, R. E., Elam, J. W., Pellin, M. J., Barke, I., von Oeynhausen, V., Lei, Y., Meyer, R. J., Alonso, J. A., Fraile Rodríguez, A., Kleibert, A., Giorgio, S., Henry, C. R., Meiwes-Broer, K.-H. \& Vajda, S. Size-dependent selectivity and activity of silver nanoclusters in the partial oxidation of propylene to propylene oxide and acrolein: A joint experimental and theoretical study. Catalysis Today 160, 116-130, doi:10.1016/j.cattod.2010.08.022 (2011).

78 Suzuki, Y., Kelly, S. D., Kemner, K. M. \& Banfield, J. F. Radionuclide contamination: Nanometre-size products of uranium bioreduction. Nature 419, 134-134 (2002).

79 Burgos, W. D., McDonough, J. T., Senko, J. M., Zhang, G., Dohnalkova, A. C., Kelly, S. D., Gorby, Y. \& Kemner, K. M. Characterization of uraninite nanoparticles produced by Shewanella oneidensis MR-1. Geochimica et Cosmochimica Acta 72, 4901-4915, doi:10.1016/j.gca.2008.07.016 (2008).

80 Marshall, M. J., Beliaev, A. S., Dohnalkova, A. C., Kennedy, D. W., Shi, L., Wang, Z., Boyanov, M. I., Lai, B., Kemner, K. M., McLean, J. S., Reed, S. B., Culley, D. E., Bailey, V. L., Simonson, C. J., Saffarini, D. A., Romine, M. F., Zachara, J. M. \& Fredrickson, J. K. c-type cytochrome-dependent formation of U(IV) nanoparticles by Shewanella oneidensis. PLoS Biol 4, e268, doi:10.1371/journal.pbio.0040268 (2006).

81 Marshall, M. J., Dohnalkova, A. C., Kennedy, D. W., Plymale, A. E., Thomas, S. H., Löffler, F. E., Sanford, R. A., Zachara, J. M., Fredrickson, J. K. \& Beliaev, A. S. Electron donor-dependent radionuclide reduction and nanoparticle formation by Anaeromyxobacter dehalogenans strain $2 \mathrm{CP}-\mathrm{C}$. Environmental Microbiology 11, 534-543, doi:10.1111/j.1462-2920.2008.01795.x (2009).

82 Wang, H. Formation of nascent soot and other condensed-phase materials in flames. Proceedings of the Combustion Institute 33, 41-67, doi:10.1016/j.proci.2010.09.009 (2011).

83 Hibara, A., Saito, T., Kim, H.-B., Tokeshi, M., Ooi, T., Nakao, M. \& Kitamori, T. Nanochannels on a Fused-Silica Microchip and Liquid Properties Investigation by Time-Resolved Fluorescence Measurements. Analytical Chemistry 74, 6170-6176, doi:10.1021/ac025808b (2002).

84 Maurice, P. A. \& Hochella, M. F. in Advances in Agronomy Vol. Volume 100 (ed L. Sparks Donald) 123-153 (Academic Press, 2008).

85 Mudunkotuwa, I. A. \& Grassian, V. H. The devil is in the details (or the surface): impact of surface structure and surface energetics on understanding the behavior of nanomaterials in the environment. Journal of Environmental Monitoring 13, 1135-1144 (2011).

86 Cölfen, H. \& Antonietti, M. Mesocrystals: Inorganic Superstructures Made by Highly Parallel Crystallization and Controlled Alignment. Angewandte Chemie International Edition 44, 5576-5591, doi:10.1002/anie.200500496 (2005).

87 Niederberger, M. \& Colfen, H. Oriented attachment and mesocrystals: Non-classical crystallization mechanisms based on nanoparticle assembly. Physical Chemistry Chemical Physics 8, 3271-3287 (2006).

88 Wigginton, N. S., Haus, K. L. \& Hochella Jr, M. F. Aquatic environmental nanoparticles. Journal of Environmental Monitoring 9, 1306-1316 (2007). 
89 De Yoreo, J. J., Zepeda-Ruiz, L. A., Friddle, R. W., Qiu, S. R., Wasylenki, L. E., Chernov, A. A., Gilmer, G. H. \& Dove, P. M. Rethinking Classical Crystal Growth Models through Molecular Scale Insights: Consequences of Kink-Limited Kinetics. Crystal Growth \& Design 9, 5135-5144, doi:10.1021/cg900543g (2009).

90 Baker, B. J., Comolli, L. R., Dick, G. J., Hauser, L. J., Hyatt, D., Dill, B. D., Land, M. L., VerBerkmoes, N. C., Hettich, R. L. \& Banfield, J. F. Enigmatic, ultrasmall, uncultivated Archaea. Proceedings of the National Academy of Sciences 107, 8806-8811, doi:10.1073/pnas.0914470107 (2010).

91 Chun, C. L., Baer, D. R., Matson, D. W., Amonette, J. E. \& Penn, R. L. Characterization and Reactivity of Iron Nanoparticles prepared with added Cu, Pd, and Ni. Environmental Science \& Technology 44, 5079-5085, doi:10.1021/es903278e (2010).

92 Banfield, J. F., Welch, S. A., Zhang, H., Ebert, T. T. \& Penn, R. L. Aggregation-Based Crystal Growth and Microstructure Development in Natural Iron Oxyhydroxide Biomineralization Products. Science 289, 751-754, doi:10.1126/science.289.5480.751 (2000).

93 Zheng, H. M., Rivest, J. B., Miller, T. A., Sadtler, B., Lindenberg, A., Toney, M. F., Wang, L. W., Kisielowski, C. \& Alivisatos, A. P. Observation of Transient Structural-Transformation Dynamics in a $\mathrm{Cu}(2) S$ Nanorod. Science 333, 206-209, doi:10.1126/science.1204713 (2011).

94 Hamm, L. M., Wallace, A. F. \& Dove, P. M. Molecular Dynamics of Ion Hydration in the Presence of Small Carboxylated Molecules and Implications for Calcification. The Journal of Physical Chemistry B 114, 10488-10495, doi:10.1021/jp9108893 (2010).

95 Penn, R. L. \& Banfield, J. F. Oriented attachment and growth, twinning, polytypism, and formation of metastable phases: Insights from nanocrystalline TiO2. A. Mineral. 83, 1077-1082 (1998).

96 Comolli, L. R., Baker, B. J., Downing, K. H., Siegerist, C. E. \& Banfield, J. F. Three-dimensional analysis of the structure and ecology of a novel, ultra-small archaeon. ISME J3, 159-167, doi:http://www.nature.com/ismej/journal/v3/n2/suppinfo/ismej200899s1.html (2008).

97 Hochella, M. F., Lower, S. K., Maurice, P. A., Penn, R. L., Sahai, N., Sparks, D. L. \& Twining, B. S. Nanominerals, Mineral Nanoparticles, and Earth Systems. Science 319, 1631-1635, doi:10.1126/science.1141134 (2008).

98 Laskin, A. Fundamentals and Applications in Aerosol Spectroscopy: Electron Beam Analysis and Microscopy of Individual Particles. 463-491 (CRC Press Taylor and Francis Group, 2010).

99 Penn, R. L. \& Banfield, J. F. Imperfect Oriented Attachment: Dislocation Generation in Defect-Free Nanocrystals. Science 281, 969-971, doi:10.1126/science.281.5379.969 (1998).

100 Moffet, R. C. T., A. V.; Gilles, M. K. in Fundamentals and Applications in Aerosol Spectroscopy (ed R. Signorell and J. Reid) 419-462 (CRC Press Taylor and Francis Group, 2010).

101 Peralta-Videa, J. R., Zhao, L., Lopez-Moreno, M. L., de la Rosa, G., Hong, J. \& Gardea-Torresdey, J. L. Nanomaterials and the environment: A review for the biennium 2008-2010. Journal of Hazardous Materials 186, 1-15, doi:10.1016/j.jhazmat.2010.11.020 (2011).

102 Penn, R. L., Tanaka, K. \& Erbs, J. Size dependent kinetics of oriented aggregation. Journal of Crystal Growth 309, 97-102, doi:10.1016/j.jcrysgro.2007.09.011 (2007).

103 Huang, H., Pierstorff, E., Osawa, E. \& Ho, D. in IEEE Nano. 
104 Huang, H., Pierstorff, E., Osawa, E. \& Ho, D. Active Nanodiamond Hydrogels for Chemotherapeutic Delivery. Nano Letters 7, 3305-3314, doi:10.1021/n1071521o (2007).

105 Huang, H., D. Ho, and E. Osawa Nanodiamond Hydrogels as Efficient and Biocompatible Transmembrane Drug Carriers. (2007).

106 Liu, J., Aruguete, D. M., Murayama, M. \& Hochella, M. F. Influence of Size and Aggregation on the Reactivity of an Environmentally and Industrially Relevant Nanomaterial (PbS). Environmental Science \& Technology 43, 8178-8183, doi:10.1021/es902121r(2009).

107 DOE_Workshop. Basic Research Needs: Catalysis for Energy. (DOE Basic Energy Sciences 2004), $<$ http://www.aps.org/meetings/multimedia/upload/Mildred_Dresselhaus.pdf. $>$ (2004).

108 Kwak, J. H., Mei, D., Yi, C.-W., Kim, D. H., Peden, C. H. F., Allard, L. F. \& Szanyi, J. Understanding the nature of surface nitrates in $\mathrm{BaO} / \gamma$-A12O3 NOx storage materials: A combined experimental and theoretical study. Journal of Catalysis 261, 17-22, doi:10.1016/j.jcat.2008.10.016 (2009).

109 Mehraeen, S., Kulkarni, A., Chi, M., Reed, B. W., Okamoto, N. L., Browning, N. D. \& Gates, B. C. Triosmium Clusters on a Support: Determination of Structure by X-ray Absorption Spectroscopy and High-Resolution Microscopy. Chemistry - A European Journal 17, 1000-1008, doi:10.1002/chem.201000860 (2011).

110 Kulkarni, A., Chi, M., Ortalan, V., Browning, N. D. \& Gates, B. C. Atomic Resolution of the Structure of a Metal-Support Interface: Triosmium Clusters on $\mathrm{MgO}(110)$. Angewandte Chemie International Edition 49, 10089-10092, doi:10.1002/anie.201005105 (2010).

111 Sharma, R., Moore, E., Rez, P. \& Treacy, M. M. J. Site-Specific Fabrication of Fe Particles for Carbon Nanotube Growth. Nano Letters 9, 689-694, doi:10.1021/n1803180e (2009).

112 Hansen, P. L., Wagner, J. B., Helveg, S., Rostrup-Nielsen, J. R., Clausen, B. S. \& Topsøe, H. AtomResolved Imaging of Dynamic Shape Changes in Supported Copper Nanocrystals. Science 295, 2053 2055, doi:10.1126/science.1069325 (2002).

113 Vargas, A., Santarossa, G., Iannuzzi, M. \& Baiker, A. Fluxionality of gold nanoparticles investigated by Born-Oppenheimer molecular dynamics. Physical Review B 80, 195421 (2009).

114 Zhou, X., Xu, W., Liu, G., Panda, D. \& Chen, P. Size-Dependent Catalytic Activity and Dynamics of Gold Nanoparticles at the Single-Molecule Level. Journal of the American Chemical Society 132, 138146, doi:10.1021/ja904307n (2009).

115 Sharma, R., Crozier $\uparrow$, P. A., Kang, Z. C. \& Eyring, L. Observation of dynamic nanostructural and nanochemical changes in ceria-based catalysts during in-situ reduction. Philosophical Magazine 84, 2731-2747, doi:10.1080/14786430410001671467 (2004).

116 Diaz, S. F., Zhu, J. F., Shamir, N. \& Campbell, C. T. Pyroelectric heat detector for measuring adsorption energies on thicker single crystals. Sensors and Actuators B: Chemical 107, 454-460, doi:10.1016/j.snb.2004.11.037 (2005).

117 Fulton, J. L., Linehan, J. C., Autrey, T., Balasubramanian, M., Chen, Y. \& Szymczak, N. K. When is a Nanoparticle a Cluster? An Operando EXAFS Study of Amine Borane Dehydrocoupling by Rh4-6 Clusters. Journal of the American Chemical Society 129, 11936-11949, doi:10.1021/ja0733311 (2007).

118 Rousseau, R., Schenter, G. K., Fulton, J. L., Linehan, J. C., Engelhard, M. H. \& Autrey, T. Defining Active Catalyst Structure and Reaction Pathways from ab Initio Molecular Dynamics and Operando 
XAFS: Dehydrogenation of Dimethylaminoborane by Rhodium Clusters. Journal of the American Chemical Society 131, 10516-10524, doi:10.1021/ja901480u (2009).

119 Stanciu, C. D. Laser-Induced Femtosecond Magnetic Recording PhD thesis, Radboud University Nijmegen, (2008).

120 Front cover. Physical Chemistry Chemical Physics 8, 5581-5581 (2006).

121 Kauffmann, Y., Oh, S. H., Koch, C. T., Hashibon, A., Scheu, C., Rühle, M. \& Kaplan, W. D. Quantitative analysis of layering and in-plane structural ordering at an alumina-aluminum solid-liquid interface. Acta Materialia 59, 4378-4386, doi:10.1016/j.actamat.2011.03.061 (2011).

122 Henager, C., Jr. \& Morris, J. R. Atomistic simulation of CdTe solid-liquid coexistence equilibria. Physical Review B 80, 245309 (2009).

123 Hoyt, J. J., Asta, M. \& Sun, D. Y. Molecular dynamics simulations of the crystal-melt interfacial free energy and mobility in Mo and V. Philosophical Magazine 86, 3651-3664, doi:10.1080/14786430500156625 (2006).

124 Buta, D., Asta, M. \& Hoyt, J. J. Atomistic simulation study of the structure and dynamics of a faceted crystal-melt interface. Physical Review E 78, 031605 (2008).

125 Henager, C. H. unpublished.

126 Ohtomo, A. \& Hwang, H. Y. A high-mobility electron gas at the LaAlO3/SrTiO3 heterointerface. Nature 427, 423-426 (2004).

127 Reyren, N., Thiel, S., Caviglia, A. D., Kourkoutis, L. F., Hammerl, G., Richter, C., Schneider, C. W., Kopp, T., Rüetschi, A.-S., Jaccard, D., Gabay, M., Muller, D. A., Triscone, J.-M. \& Mannhart, J. Superconducting Interfaces Between Insulating Oxides. Science 317, 1196-1199, doi:10.1126/science.1146006 (2007).

128 Ariando, Wang, X., Baskaran, G., Liu, Z. Q., Huijben, J., Yi, J. B., Annadi, A., Barman, A. R., Rusydi, A., Dhar, S., Feng, Y. P., Ding, J., Hilgenkamp, H. \& Venkatesan, T. Electronic phase separation at the LaAlO3/SrTiO3 interface. Nat Commun 2, 188 (2011).

129 Hammerl, G. \& Spaldin, N. Shedding Light on Oxide Interfaces. Science 332, 922-923, doi:10.1126/science.1206247 (2011).

130 Morkoç, H., Carlo, A. D. \& Cingolani, R. GaN-based modulation doped FETs and UV detectors. SolidState Electronics 46, 157-202, doi:10.1016/s0038-1101(01)00271-4 (2002).

131 Chambers, S. A., Engelhard, M. H., Shutthanandan, V., Zhu, Z., Droubay, T. C., Qiao, L., Sushko, P. V., Feng, T., Lee, H. D., Gustafsson, T., Garfunkel, E., Shah, A. B., Zuo, J. M. \& Ramasse, Q. M. Instability, intermixing and electronic structure at the epitaxial heterojunction. Surface Science Reports 65, 317-352, doi:10.1016/j.surfrep.2010.09.001 (2010).

132 Robertson, I. M., Lee, T. C. \& Birnbaum, H. K. Application of the in situ TEM deformation technique to observe how "clean" and doped grain boundaries respond to local stress concentrations.

Ultramicroscopy 40, 330-338, doi:10.1016/0304-3991(92)90130-c (1992).

133 Liu, Y. G., Jia, D. C., Zhou, Y., Fang, M. H. \& Huang, Z. H. In situ TEM observation of crack propagation in $\mathrm{LiTaO} 3$ particle toughened Al2O3 ceramics. Ceram. Int. 37, 647-650, doi:10.1016/j.ceramint.2010.10.002 (2011). 
134 Wang, H., Nie, A., Liu, J., Wang, P., Yang, W., Chen, B., Liu, H. \& Fu, M. In situ TEM study on crack propagation in nanoscale Au thin films. Scripta Materialia 65, 377-379, doi:10.1016/j.scriptamat.2011.05.009 (2011).

135 Haque, M. A. a. M. T. A. S. In situ Tensile Testing of Nanoscale Freestanding Thin Films Inside a Transmission Electron Microscope. Journal of Materials Research 20, 1769-1777, doi:doi:10.1557/JMR.2005.0220 (2005).

136 Rana, D. S., Kawayama, I., Mavani, K., Takahashi, K., Murakami, H. \& Tonouchi, M. Understanding the Nature of Ultrafast Polarization Dynamics of Ferroelectric Memory in the Multiferroic BiFeO3. Advanced Materials 21, 2881-2885, doi:10.1002/adma.200802094 (2009).

137 Yang, Y. C., Pan, F., Liu, Q., Liu, M. \& Zeng, F. Fully Room-Temperature-Fabricated Nonvolatile Resistive Memory for Ultrafast and High-Density Memory Application. Nano Letters 9, 1636-1643, doi:10.1021/n1900006g (2009).

138 Xue, K.-H., de Araujo, C. A. P., Celinska, J. \& McWilliams, C. A non-filamentary model for unipolar switching transition metal oxide resistance random access memories. Journal of Applied Physics 109, 091602-091606 (2011).

139 Ashton, T. S. Iron and Steel in the Industrial Revolution., (New York, A.M. Kelley, 1968).

140 Dash, J. \& Otte, H. M. The martensite transformation in stainless steel. Acta Metallurgica 11, 11691178, doi:10.1016/0001-6160(63)90044-0 (1963).

141 Olson, G. \& Cohen, M. Stress-assisted isothermal martensitic transformation: Application to TRIP steels. Metallurgical and Materials Transactions A 13, 1907-1914, doi:10.1007/bf02645934 (1982).

142 Jacques, P., Delannay, F., Cornet, X., Harlet, P. \& Ladriere, J. Enhancement of the mechanical properties of a low-carbon, low-silicon steel by formation of a multiphased microstructure containing retained Austenite. Metallurgical and Materials Transactions A 29, 2383-2393, doi:10.1007/s11661998-0114-1 (1998).

143 Hamann, H. F., O'Boyle, M., Martin, Y. C., Rooks, M. \& Wickramasinghe, H. K. Ultra-high-density phase-change storage and memory. Nat Mater 5, 383-387, doi:http://www.nature.com/nmat/journal/v5/n5/suppinfo/nmat1627_S1.html (2006).

144 Miyazaki, S. Medical and Dental Applications of Shape Memory Alloys. 267-281 (Cambridge University Press, 1998).

145 Olsen, G. B. Introduction: Martensite in perspective. 1-10 (ASM International, 1992).

146 Boettger, J. C. \& Wallace, D. C. Metastability and dynamics of the shock-induced phase transition in iron. Physical Review B 55, 2840-2849 (1997).

147 Porter, D. A. \& Easterling, K. E. Phase transformaiton in metals and alloys. (Van Nostrand Reinhold Co., 1981).

148 Doherty, R. D. Nucleation. 23-61 (Riederer-Verlag, 1978).

149 Wayman, C. M. \& Bhadeshia, H. K. D. H. Phase Transformations, Nondiffusive. (North-Holland, 1996).

150 Kaufman, L. \& Cohen, M. Thermodynamics and kinetics of martensitic transformations. Progress in Metal Physics 7, 165-246, doi:10.1016/0502-8205(58)90005-4 (1958). 
151 Cech, R. E. a. D. T. Heterogeneous Nucleation Of The Martensite Transformation. Transactions Of The American Institute Of Mining And Metallurgical Engineers 206, 124-132 (1956).

152 Magee, C. L. The nucleation of Martensite. 115-156 (America Society for Metals, 1970).

153 Zhang, L., Zhou, T., Aindow, M., Alpay, S., Blackburn, M. \& Wu, M. Nucleation of stress-induced martensites in a Ti/Mo-based alloy. Journal of Materials Science 40, 2833-2836, doi:10.1007/s10853005-2426-5 (2005).

154 LeGoues, F. K. \& Aaronson, H. I. Influence of crystallography upon critical nucleus shapes and kinetics of homogeneous f.c.c.-f.c.c. nucleation-IV. Comparisons between theory and experiment in $\mathrm{CuCo}$ alloys. Acta Metallurgica 32, 1855-1864, doi:10.1016/0001-6160(84)90243-8 (1984).

155 Offerman, S. E., van Dijk, N. H., Sietsma, J., Grigull, S., Lauridsen, E. M., Margulies, L., Poulsen, H. F., Rekveldt, M. T. \& van der Zwaag, S. Grain Nucleation and Growth During Phase Transformations. Science 298, 1003-1005, doi:10.1126/science.1076681 (2002).

156 van Dijk, N. H., Offerman, S. E., Sietsma, J. \& van der Zwaag, S. Barrier-free heterogeneous grain nucleation in polycrystalline materials: The austenite to ferrite phase transformation in steel. Acta Materialia 55, 4489-4498, doi:10.1016/j.actamat.2007.04.013 (2007).

157 Aaronson, H. I., Lange, I. \& Purdy, G. R. Discussion to "Grain nucleation and growth during phase transformations" by S.E. Offerman et al., Science, 298, 1003 (November 1, 2002). Scripta Materialia 51, 931-935, doi:10.1016/j.scriptamat.2004.06.030 (2004).

158 Offerman, S. E., Dijk, v., Sietsma, J., der Zwaag, v., Lauridsen, E. M., Margulies, L., Grigull, S. \& Poulsen, H. F. Reply to the discussion by Aaronson et al. to "Grain nucleation and growth during phase transformations" by S.E. Offerman et al., Science, 298, 1003 (November 1, 2002). Scripta Materialia 51, 937-941, doi:10.1016/j.scriptamat.2004.06.031 (2004).

159 Xiao, S. Q. \& Haasen, P. HREM investigation of homogeneous decomposition in a Ni-12 at. \% A1 Alloy. Acta Metallurgica et Materialia 39, 651-659, doi:10.1016/0956-7151(91)90133-1 (1991).

160 Aaronson, H. I. a. K. C. R. Nucleation - mostly homogeneous and in solids. 371-397 (Met. Soc. AIME, 1982).

161 Pond, R. C., Shang, P., Cheng, T. T. \& Aindow, M. Interfacial dislocation mechanism for diffusional phase transformations exhibiting martensitic crystallography: formation of TiAl + Ti3Al lamellae. Acta Materialia 48, 1047-1053, doi:10.1016/s1359-6454(99)00416-4 (2000).

162 Mackenzie, J. K. \& Bowles, J. S. The crystallography of martensite transformations II. Acta Metallurgica 2, 138-147, doi:10.1016/0001-6160(54)90103-0 (1954).

163 Bowles, J. S. \& Mackenzie, J. K. The crystallography of martensite transformations I. Acta Metallurgica 2, 129-137, doi:10.1016/0001-6160(54)90102-9 (1954).

164 Hirth, J. P. \& Pond, R. C. Steps, dislocations and disconnections as interface defects relating to structure and phase transformations. Acta Materialia 44, 4749-4763, doi:10.1016/s1359-6454(96)00132-2 (1996).

165 Vitek, V. Theory of the Core Structure of Dislocations in Body - Centered - Cubic Metals. Crystal Lattice Defects 5, 1-34 (1974).

166 Fleming, G., M. Ratner. Directing Matter and Energy: Five Challenges for Science and the Imagination. (Basic Energy Sciences Advisory Committee, Office of Science, Department of Energy 2007), 2007. 
167 Dagotto, E. Complexity in Strongly Correlated Electronic Systems. Science 309, 257-262, doi:10.1126/science.1107559 (2005).

168 Fausti, D., Tobey, R. I., Dean, N., Kaiser, S., Dienst, A., Hoffmann, M. C., Pyon, S., Takayama, T., Takagi, H. \& Cavalleri, A. Light-Induced Superconductivity in a Stripe-Ordered Cuprate. Science 331, 189-191, doi:10.1126/science.1197294 (2011).

169 Chuang, T.-M., Allan, M. P., Lee, J., Xie, Y., Ni, N., Bud'ko, S. L., Boebinger, G. S., Canfield, P. C. \& Davis, J. C. Nematic Electronic Structure in the "Parent" State of the Iron-Based Superconductor $\mathrm{Ca}(\mathrm{Fe} 1-\mathrm{xCox}) 2 \mathrm{As} 2$. Science 327, 181-184, doi:10.1126/science.1181083 (2010).

170 Lang, K. M., Madhavan, V., Hoffman, J. E., Hudson, E. W., Eisaki, H., Uchida, S. \& Davis, J. C. Imaging the granular structure of high-Tc superconductivity in underdoped $\mathrm{Bi} 2 \mathrm{Sr} 2 \mathrm{CaCu} 2 \mathrm{O} 8+[$ delta]. Nature 415, 412-416 (2002).

171 Markland, T. E., Morrone, J. A., Berne, B. J., Miyazaki, K., Rabani, E. \& Reichman, D. R. Quantum fluctuations can promote or inhibit glass formation. Nat Phys 7, 134-137, doi:http://www.nature.com/nphys/journal/v7/n2/abs/nphys 1865.html\#supplementary-information (2011).

172 Carbone, F., Kwon, O.-H. \& Zewail, A. H. Dynamics of Chemical Bonding Mapped by EnergyResolved 4D Electron Microscopy. Science 325, 181-184, doi:10.1126/science.1175005 (2009).

173 Tobey, R. I., Prabhakaran, D., Boothroyd, A. T. \& Cavalleri, A. Ultrafast Electronic Phase Transition in La_\{1/2\}Sr_\{3/2\}MnO_\{4\} by Coherent Vibrational Excitation: Evidence for Nonthermal Melting of Orbital Order. Physical Review Letters 101, 197404 (2008).

174 Odette, G. R., Alinger, M. J. \& Wirth, B. D. Recent Developments in Irradiation-Resistant Steels. Annual Review of Materials Research 38, 471-503, doi:10.1146/annurev.matsci.38.060407.130315 (2008).

175 Ukai, S. \& Fujiwara, M. Perspective of ODS alloys application in nuclear environments. Journal of Nuclear Materials 307-311, Part 1, 749-757, doi:10.1016/s0022-3115(02)01043-7 (2002).

176 Akasaka, N., Yamashita, S., Yoshitake, T., Ukai, S. \& Kimura, A. Microstructural changes of neutron irradiated ODS ferritic and martensitic steels. Journal of Nuclear Materials 329-333, Part B, 10531056, doi:10.1016/j.jnucmat.2004.04.133 (2004).

177 Klimiankou, M., Lindau, R. \& Möslang, A. TEM characterization of structure and composition of nanosized ODS particles in reduced activation ferritic-martensitic steels. Journal of Nuclear Materials 329-333, Part A, 347-351, doi:10.1016/j.jnucmat.2004.04.083 (2004).

178 Schäublin, R., Ramar, A., Baluc, N., de Castro, V., Monge, M. A., Leguey, T., Schmid, N. \& Bonjour, C. Microstructural development under irradiation in European ODS ferritic/martensitic steels. Journal of Nuclear Materials 351, 247-260, doi:10.1016/j.jnucmat.2006.02.005 (2006).

179 Alamo, A., Bertin, J. L., Shamardin, V. K. \& Wident, P. Mechanical properties of 9Cr martensitic steels and ODS-FeCr alloys after neutron irradiation at $325 \& \# x a 0 ;{ }^{\circ} \mathrm{C}$ up to $42 \& \# x a 0 ;$ dpa. Journal of Nuclear Materials 367-370, Part A, 54-59, doi:10.1016/j.jnucmat.2007.03.166 (2007).

180 Kishimoto, H., Kasada, R., Hashitomi, O. \& Kimura, A. Stability of Y-Ti complex oxides in Fe-16Cr$0.1 \mathrm{Ti}$ ODS ferritic steel before and after heavy-ion irradiation. Journal of Nuclear Materials 386-388, 533-536, doi:10.1016/j.jnucmat.2008.12.169 (2009). 
181 Kovarik, L. unpublished results.

182 Was, G. S. Fundamentals of Radiation Materials Science. (Springer, 2007).

183 Edwards, D. J. unpublished results.

184 Banhart, F., Kotakoski, J. \& Krasheninnikov, A. V. Structural Defects in Graphene. ACS Nano 5, 26-41, doi:10.1021/nn102598m (2010).

185 Baum, P., Yang, D.-S. \& Zewail, A. H. 4D Visualization of Transitional Structures in Phase Transformations by Electron Diffraction. Science 318, 788-792, doi:10.1126/science.1147724 (2007).

186 Yang, D.-S., Lao, C. \& Zewail, A. H. 4D Electron Diffraction Reveals Correlated Unidirectional Behavior in Zinc Oxide Nanowires. Science 321, 1660-1664, doi:10.1126/science.1162049 (2008).

187 Kwon, O.-H., Barwick, B., Park, H. S., Baskin, J. S. \& Zewail, A. H. Nanoscale Mechanical Drumming Visualized by 4D Electron Microscopy. Nano Letters 8, 3557-3562, doi:10.1021/n18029866 (2008).

188 Oh, S. H., Kauffmann, Y., Scheu, C., Kaplan, W. D. \& Rühle, M. Ordered Liquid Aluminum at the Interface with Sapphire. Science 310, 661-663, doi:10.1126/science.1118611 (2005).

189 Radisic, A., Vereecken, P. M., Hannon, J. B., Searson, P. C. \& Ross, F. M. Quantifying Electrochemical Nucleation and Growth of Nanoscale Clusters Using Real-Time Kinetic Data. Nano Letters 6, 238-242, doi:10.1021/n1052175i (2006).

190 Minor, A. M., Lilleodden, E. T., Stach, E. A. \& Morris, J. W. Direct observations of incipient plasticity during nanoindentation of Al. Journal of Materials Research 19, 176-182, doi:doi:10.1557/jmr.2004.19.1.176 (2004).

191 Zhou, G. \& Yang, J. C. Initial Oxidation Kinetics of $\mathrm{Cu}(100)$, (110), and (111) Thin Films Investigated by in Situ Ultra-high-vacuum Transmission Electron Microscopy. Journal of Materials Research 20, 1684-1694, doi:doi:10.1557/JMR.2005.0239 (2005).

192 Sharma, R. An Environmental Transmission Electron Microscope for in situ Synthesis and Characterization of Nanomaterials. Journal of Materials Research 20, 1695-1707, doi:doi:10.1557/JMR.2005.0241 (2005).

193 Reed, B. W., LaGrange, T., Shuttlesworth, R. M., Gibson, D. J., Campbell, G. H. \& Browning, N. D. Solving the accelerator-condenser coupling problem in a nanosecond dynamic transmission electron microscope. Review of Scientific Instruments 81, 053706-053708 (2010).

194 Taheri, M. L., Reed, B. W., LaGrange, T. B. \& Browning, N. D. In Situ Laser Synthesis of Si Nanowires in the Dynamic TEM. Small 4, 2187-2190, doi:10.1002/smll.200800588 (2008).

195 Armstrong, M. R., Reed, B. W., Torralva, B. R. \& Browning, N. D. Prospects for electron imaging with ultrafast time resolution. Applied Physics Letters 90, 114101-114103 (2007).

196 Armstrong, M. R., Boyden, K., Browning, N. D., Campbell, G. H., Colvin, J. D., DeHope, W. J., Frank, A. M., Gibson, D. J., Hartemann, F., Kim, J. S., King, W. E., LaGrange, T. B., Pyke, B. J., Reed, B. W., Shuttlesworth, R. M., Stuart, B. C. \& Torralva, B. R. Practical considerations for high spatial and temporal resolution dynamic transmission electron microscopy. Ultramicroscopy 107, 356-367, doi:10.1016/j.ultramic.2006.09.005 (2007).

197 Bostanjoglo, O., Elschner, R., Mao, Z., Nink, T. \& Weingärtner, M. Nanosecond electron microscopes. Ultramicroscopy 81, 141-147, doi:10.1016/s0304-3991(99)00180-1 (2000). 
198 Bostanjoglo, O. in Advances in Imaging and Electron Physics Vol. 121 (ed W. Hawkes Peter) 1-51 (Elsevier, 2002).

199 Rose, H. \& Spehr, R. in Advances in Electronics and Electron Physics Vol. V59 475-530 (Academic Press, 1983).

200 Reiser, M. Theory and Design of Charged Particle Beams. 1 edn, (Wiley-VCH, 1994).

201 Kruit, P. \& Jansen, G. H. in Handbook of Charged Particle Optics $\quad$ 275-318 (CRC Press, 1997).

202 Siwick, B. J., Dwyer, J. R., Jordan, R. E. \& Miller, R. J. D. Ultrafast electron optics: Propagation dynamics of femtosecond electron packets. Journal of Applied Physics 92, 1643-1648 (2002).

203 Reed, B. W. Femtosecond electron pulse propagation for ultrafast electron diffraction. Journal of Applied Physics 100, 034916-034916 (2006).

204 Michalik, A. M. \& Sipe, J. E. Analytic model of electron pulse propagation in ultrafast electron diffraction experiments. Journal of Applied Physics 99, 054908-054909 (2006).

205 Gahlmann, A., Tae Park, S. \& Zewail, A. H. Ultrashort electron pulses for diffraction, crystallography and microscopy: theoretical and experimental resolutions. Physical Chemistry Chemical Physics 10, 2894-2909 (2008).

206 Vellekoop, I. M., van Putten, E. G., Lagendijk, A. \& Mosk, A. P. Demixing light paths inside disordered metamaterials. Opt. Express 16, 67-80 (2008).

207 van Oudheusden, T., de Jong, E. F., van der Geer, S. B., t Root, W. P. E. M. O., Luiten, O. J. \& Siwick, B. J. Electron source concept for single-shot sub-100 fs electron diffraction in the $100 \mathrm{keV}$ range. Journal of Applied Physics 102, 093501-093508 (2007).

208 Krivanek, O. L. High-Resolution Transmission Electron Microscopy and Associated Techniques. 519567 (Oxford University Press, 1992).

209 Rose, A. Advances in Electronics and Electron Physics. (Academic Press, 1948).

210 Kisielowski, C., Freitag, B., Bischoff, M., van Lin, H., Lazar, S., Knippels, G., Tiemeijer, P., van der Stam, M., von Harrach, S., Stekelenburg, M., Haider, M., Uhlemann, S., Müller, H., Hartel, P., Kabius, B., Miller, D., Petrov, I., Olson, E. A., Donchev, T., Kenik, E. A., Lupini, A. R., Bentley, J., Pennycook, S. J., Anderson, I. M., Minor, A. M., Schmid, A. K., Duden, T., Radmilovic, V., Ramasse, Q. M., Watanabe, M., Erni, R., Stach, E. A., Denes, P. \& Dahmen, U. Detection of Single Atoms and Buried Defects in Three Dimensions by Aberration-Corrected Electron Microscope with 0.5- $\AA$ Information Limit. Microscopy and Microanalysis 14, 469-477, doi:doi:10.1017/S1431927608080902 (2008).

211 King, W. E., Campbell, G. H., Frank, A., Reed, B., Schmerge, J. F., Siwick, B. J., Stuart, B. C. \& Weber, P. M. Ultrafast electron microscopy in materials science, biology, and chemistry. Journal of Applied Physics 97, 111101-111127 (2005).

212 Valfells, A., Feldman, D. W., Virgo, M., O'Shea, P. G. \& Lau, Y. Y. Effects of pulse-length and emitter area on virtual cathode formation in electron guns. Physics of Plasmas 9, 2377-2382 (2002).

213 Zawadzka, J., Jaroszynski, D. A., Carey, J. J. \& Wynne, K. Evanescent-wave acceleration of ultrashort electron pulses. Applied Physics Letters 79, 2130-2132 (2001). 
214 Kupersztych, J., Monchicourt, P. \& Raynaud, M. Ponderomotive Acceleration of Photoelectrons in Surface-Plasmon-Assisted Multiphoton Photoelectric Emission. Physical Review Letters 86, 5180-5183 (2001).

215 Schroeder, W., Berger, J., Nicholls, A., Zaluzec, N., Hiller, J. \& Miller, D. High Brightness NanoPatterned Photocathode Electron Sources for UEM. Microscopy and Microanalysis 16, 492-493, doi:doi:10.1017/S1431927610059672 (2010).

216 Geppert, I., Eizenberg, M., Ali, A. \& Datta, S. Band offsets determination and interfacial chemical properties of the Al[sub 2]O[sub 3]/GaSb system. Applied Physics Letters 97, 162109-162103 (2010).

217 Liang, J., Chik, H. \& Xu, J. Nonlithographic fabrication of lateral superlattices for nanometric electromagnetic-optic applications. Selected Topics in Quantum Electronics, IEEE Journal of 8, 9981008 (2002).

218 Jensen, K. L., O'Shea, P. G., Feldman, D. W. \& Moody, N. A. Theoretical model of the intrinsic emittance of a photocathode. Applied Physics Letters 89, 224103-224103 (2006).

219 Strickland, D. \& Mourou, G. Compression of amplified chirped optical pulses. Optics Communications 56, 219-221, doi:10.1016/0030-4018(85)90120-8 (1985).

220 Crane, J. K., Martinez, M., Beach, R. J., Mitchell, S., Pratt, G. \& Christensen, J. J. in CLEO '96.

221 Hanson, F. \& Imthurn, G. Efficient laser diode side pumped neodymium glass slab laser. Quantum Electronics, IEEE Journal of 24, 1811-1813 (1998).

222 Bagnoud, V., Luce, J., Videau, L. \& Rouyer, C. Diode-pumped regenerative amplifier delivering 100mJ single-mode laser pulses. Opt. Lett. 26, 337-339 (2001).

223 Grünig, M., Staub, F. \& Balmer, J. E. Nd:glass regenerative amplifier for CPA (chirped-pulse amplification) lasers. Optics and Lasers in Engineering 43, 263-269, doi:10.1016/j.optlaseng.2004.04.006 (2005).

224 Berger, J., Hogan, J. T., Greco, M. J., Schroeder, W., Nicholls, A. W. \& Browning, N. D. DC Photoelectron Gun Parameters for Ultrafast Electron Microscopy. Microscopy and Microanalysis 15, 298-313, doi:doi:10.1017/S1431927609090266 (2009).

225 Galvanauskas, A., Cho, G. C., Hariharan, A., Fermann, M. E. \& Harter, D. Generation of high-energy femtosecond pulses in multimode-core Yb-fiber chirped-pulse amplification systems. Opt. Lett. 26, 935937 (2001).

226 Homann, C., Schriever, C., Baum, P. \& Riedle, E. Octave wide tunable UV-pumped NOPA: pulses down to $20 \mathrm{fs}$ at $0.5 \mathrm{MHz}$ repetition rate. Opt. Express 16, 5746-5756 (2008).

227 Veisz, L., Kurkin, G., Chernov, K., Tarnetsky, V., Apolonski, A., Krausz, F. \& Fill, E. Hybrid dc-ac electron gun for fs-electron pulse generation. New Journal of Physics 9, 451-468 (2007).

228 van Oudheusden, T., Pasmans, P. L. E. M., van der Geer, S. B., de Loos, M. J., van der Wiel, M. J. \& Luiten, O. J. Compression of Subrelativistic Space-Charge-Dominated Electron Bunches for Single-Shot Femtosecond Electron Diffraction. Physical Review Letters 105, 264801 (2010).

229 Berger, J. A. \& Schroeder, W. A. Semianalytic model of electron pulse propagation: Magnetic lenses and rf pulse compression cavities. Journal of Applied Physics 108, 124905-124912 (2010). 
230 Ishizuka, K. Contrast transfer of crystal images in TEM. Ultramicroscopy 5, 55-65, doi:10.1016/03043991(80)90011-x (1980).

231 Scherzer, O. Optik 2, 114-132 (1947).

232 van Benthem, K., Lupini, A. R., Kim, M., Baik, H. S., Doh, S., Lee, J.-H., Oxley, M. P., Findlay, S. D., Allen, L. J., Luck, J. T. \& Pennycook, S. J. Three-dimensional imaging of individual hafnium atoms inside a semiconductor device. Applied Physics Letters 87, 034104-034103 (2005).

233 Cosgriff, E. C., D’Alfonso, A. J., Allen, L. J., Findlay, S. D., Kirkland, A. I. \& Nellist, P. D. Threedimensional imaging in double aberration-corrected scanning confocal electron microscopy, Part I:: Elastic scattering. Ultramicroscopy 108, 1558-1566, doi:10.1016/j.ultramic.2008.05.009 (2008).

234 Barwick, B. \& Batelaan, H. Aharonov-Bohm phase shifts induced by laser pulses. New Journal of Physics 10, 083036 (2008).

235 Krausz, F. \& Ivanov, M. Attosecond physics. Reviews of Modern Physics 81, 163-234 (2009).

236 Brabec, T. \& Krausz, F. Intense few-cycle laser fields: Frontiers of nonlinear optics. Reviews of Modern Physics 72, 545-591 (2000).

237 Tzallas, P., Tsakiris, G. D., Witte, K., Nikolopoulos, L. A. A., Benis, E. P. \& Charalambidis, D. The attosecond-science frontiers: generation, metrology and paths to applications. Journal of Electron Spectroscopy and Related Phenomena 144-147, 1129-1135, doi:10.1016/j.elspec.2005.01.267 (2005).

238 Sundaram, S. K. in American Association of Advancement of Science (AAAS) Annual Meeting.

239 Bandrauk, A. D., Fisch, N. J. \& Starace, A. F. Attosecond Science Workshop (The Kavli Institute for Theorectical Physics, 2006).

240 Corkum, P. B. \& Krausz, F. Attosecond science. Nat Phys 3, 381-387 (2007).

241 Tempea, G., a. Stingl. Ultrafast optics: dielectric multilayer mirrors enable shortest pulse lengths. Laser Focus World 43 (2007).

242 Kling, M. F. \& Vrakking, M. J. J. Attosecond Electron Dynamics. Annual Review of Physical Chemistry 59, 463-492, doi:10.1146/annurev.physchem.59.032607.093532 (2008).

243 Attwood, D., w. Barletta, F. Parmigani, and C. Pellegrini. Workshop on "X-ray Science at the Femtosecond to Attosecond Frontier (UCLA, Los Angeles, 2009).

244 Bandrauk, A. D., K. L. Ishikawa, J-M. Rost and A. F. Starace. Attosecond Science - Exploring and Controlling Matter on Its Natural Time Scale (Chinese Academy of Sciences, 2011).

245 Mourou, G. \& Tajima, T. More Intense, Shorter Pulses. Science 331, 41-42, doi:10.1126/science.1200292 (2011).

246 Ipp, A. in International Conference on Physics in Intense Fields. 4.

247 Stockman, M. I., Kling, M. F., Kleineberg, U. \& Krausz, F. Attosecond nanoplasmonic-field microscope. Nat Photon 1, 539-544, doi:http://www.nature.com/nphoton/journal/v1/n9/suppinfo/nphoton.2007.169 S1.html (2007).

248 Hommelhoff, P., Sortais, Y., Aghajani-Talesh, A. \& Kasevich, M. A. Field Emission Tip as a Nanometer Source of Free Electron Femtosecond Pulses. Physical Review Letters 96, 077401 (2006). 
249 Shorokhov, D. \& Zewail, A. H. 4D electron imaging: principles and perspectives. Physical Chemistry Chemical Physics 10, 2879-2893 (2008).

250 Fill, E., Veisz, L., Apolonski, A. \& Krausz, F. Sub-fs electron pulses for ultrafast electron diffraction. New Journal of Physics 8, 272 (2006).

251 Plettner, T., Lu, P. P. \& Byer, R. L. Proposed few-optical cycle laser-driven particle accelerator structure. Physical Review Special Topics - Accelerators and Beams 9, 111301 (2006).

252 Ipp, A., Evers, J., Keitel, C. H. \& Hatsagortsyan, K. Z. Streaking at high energies with electrons and positrons. Physics Letters B 702, 383-387, doi:10.1016/j.physletb.2011.07.027 (2011).

253 Liu, Y. G., Jia, D. C., Zhou, Y., Fang, M. H. \& Huang, Z. H. In situ TEM observation of crack propagation in $\mathrm{LiTaO}(3)$ particle toughened $\mathrm{Al}(2) \mathrm{O}(3)$ ceramics. Ceram. Int. 37, 647-650, doi:10.1016/j.ceramint.2010.10.002 (2011). 


\section{Figures}

Figure 1.Scientific challenges, dynamic process, and their intrinsic time and length scales. The cuboids in the background (present in situ TEM, DTEM I, DTEM II, and UTEM) indicate which technology must be used to access certain processes. DTEM I is the dynamic transmission electron microscope (DTEM I) at Lawrence Livermore National Laboratory (LLNL). DTEM II development was started at the University of California, Davis (UC-Davis) and is currently being transferred to PNNL. The proposed, new UTEM capability will build on prior projects and will enable observation of shorter time scale processes that are beyond the reach of current capability. 14

Figure 2. The initial conformation (left) of the substrate chain (shown in red); (at right) the final conformation (shown in black). ${ }^{25}$ This is a modeled depiction, but the UTEM approach would provide actual, dynamic conformational detail of this process at the nanosecond timescale. 18

Figure 3. Grain boundary movement in Au: These high-resolution TEM images show that a grain boundary (marked by the dotted red line) moves several nanometers between two frames of the movie recorded under in situ conditions. ${ }^{26}$ The details of the mechanism of grain boundary movement are beyond the time resolution of this experiment, but could be observed using the proposed UTEM approach. 18

Figure 4: Comparison of atomic cross-sections (element = carbon) for electrons and x-rays as a function of wavelength. Absorption, Elastic and Inelastic scattering cross-sections are displayed (red, green, and blue, respectively). 20

Figure 5. High-resolution structures for $\mathrm{FDH}_{\mathrm{H}}$, , highlight ingSeCys ${ }^{140}$ (space-filling representation) on flexible loop structure $\mathrm{Val}^{133}-\mathrm{Ala}^{146}$ (red), positions of Mo (green sphere) and [4Fe/4S] cluster (yellow), and flexible loops designated for engineering probe labeling sites (green).... 7

Figure 6.Cartoon illustrating membrane proteins linked to pathway involving extracellular electron transfer to the environment (left) and schematic highlighting trafficking pathway of outer membrane decaheme cytochrome MtrC (right) (Shi et al., 2007; Xiong et al., 2011). 14

Figure 7.Nanoscale interparticle spacing formed in $\mathrm{PbS}$ nanoparticle clusters that show dissolution is slow in the confined space; (a) before and (b) after dissolution ${ }^{106}$ 18

Figure 8.(\#A) High-resolution STEM image shows active monomeric catalyst structures $(2 \% \mathrm{BaO}$ on $\gamma$ $\mathrm{Al}_{2} \mathrm{O}_{3}$ ). A: No $\mathrm{BaO}$; $\mathrm{B}, \mathrm{C}$ : fairly uniform dispersion of $\mathrm{BaO}$; Line scans 1, 2: confirms single $\mathrm{BaO}$ monomers; $\mathrm{D}$ : $\mathrm{BaO}$ monomer decorate the facet boundary. (\#B) Computed structure of a $\mathrm{BaO}$ monomer on a dehydroxylated $\gamma-\mathrm{Al}_{2} \mathrm{O}_{3}(100)$ surface (green - $\mathrm{Ba}$; dark red - $\mathrm{O}$ in $\mathrm{BaO}$; magenta $\mathrm{Al}_{3+}$ penta and red - surface $\mathrm{O}$ atom on the $\gamma-\mathrm{Al}_{2} \mathrm{O}_{3}(100)$ surface. Bulk alumina indicated by light lines. 108 20

Figure 9.Image of triosmium clusters on the surface of $\mathrm{MgO}$ powder. These clusters were prepared from $\mathrm{Os}_{3}(\mathrm{CO})_{12}$. The aberration-corrected STEM images show an average Os-Os distance in the clusters of $2.80 \pm 0.14 \AA$. The distance determined by extended $\mathrm{x}$-ray absorption spectroscopy is $2.89 \pm 0.06$ Å. 109 20

Figure 10.Aberration-corrected STEM image showing individual Os atoms and Os atoms in triosmium clusters located atop $\mathrm{Mg}$ atoms on $\mathrm{Mg}(110)$. The encircled areas show intact triosmium clusters. A 
is the image. $\mathrm{B}$ is a schematic representation of the image showing various orientations of the clusters on the support surface. ${ }^{110}$ 21

Figure 11. Two structural models of supported triosmium clusters on $\mathrm{MgO}$ (110) depicting the Ossupport interactions on hydroxylated $\mathrm{MgO}$ (110) and on dehydroxylated $\mathrm{MgO}$ (110) (side and perspective views). (A) Triosmium cluster orientation I on dehydroxylated $\mathrm{MgO}(110)$. (B) Triosmium cluster orientation I on hydroxylated $\mathrm{MgO}(110)(\mathrm{C})$ Triosmium cluster orientation II, including two models for dehydroxylated $\mathrm{MgO}$ (110) and (D) triosmium cluster orientation II, including two models for hydroxylated $\mathrm{MgO}(110)$. The hydroxylated surfaces consist of hydroxyl groups on surface $\mathrm{Mg}$ atoms and protons on neighboring surface $\mathrm{O}$ atoms. The structure and bonding distances of adsorbed hydroxyls and hydrogen bonding between $\mathrm{O}$ of hydroxyl and protons on surface $\mathrm{O}$ atoms were estimated on the basis of reported density functional theory (DFT)

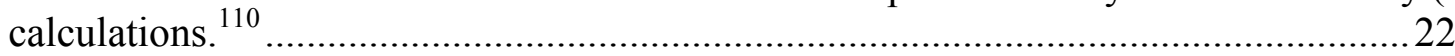

Figure 12.Approximate time scales relevant to catalysis and the corresponding UTEM spatial resolution required to image the processes. Bond breaking and formation are ultrafast (fs to ps) processes that require angstrom-scale imaging resolution. Nucleation and growth of the catalyst occur roughly on the ps and ns time scales, respectively, and require $\mathrm{nm}$ to micron spatial resolution. Fluxionality (changes in local morphology of the catalyst) occurs typically on time scales ranging from picoseconds to seconds. Full catalytic cycles typically occur on the order of seconds, and deactivation of catalysts takes place on time scales ranging from seconds to years....23

Figure 13. Static images extracted from a digital video recorded at $650^{\circ} \mathrm{C}$ as an iron particle was exposed to $10 \mathrm{~m}$ Torr of flowing acetylene with a time resolution of 9 frames $\mathrm{s}^{-1}$. The phase transformation of $\mathrm{BCC}$ iron into $\mathrm{Fe}_{3} \mathrm{C}$ occurred within $0.11 \mathrm{~s}$, the time resolution of the digital recording, before the growth of carbon nanotubes shown at lower right. ${ }^{111}$ 24

Figure 14.In situ TEM images $(\mathrm{A}, \mathrm{C}$, and $\mathrm{E}$ ) of a $\mathrm{Cu} / \mathrm{ZnO}$ catalyst in various gas environments together with the corresponding Wulff constructions of the $\mathrm{Cu}$ nanocrystals $(\mathrm{B}, \mathrm{D}$, and $\mathrm{F})$. (A) The image was recorded at a pressure of $1.5 \mathrm{mbar}^{\circ} \mathrm{H}_{2}$ at $220^{\circ} \mathrm{C}$. The electron beam is parallel to the [011] zone axis of copper. (C) Obtained in a gas mixture of $\mathrm{H}_{2}$ and $\mathrm{H}_{2} \mathrm{O}, \mathrm{H}_{2}: \mathrm{H}_{2} \mathrm{O} 5$ 3:1 at a total pressure of $1.5 \mathrm{mbar}$ at $220^{\circ} \mathrm{C}$. (E) Obtained in a gas mixture of $\mathrm{H}_{2}(95 \%)$ and $\mathrm{CO}(5 \%)$ at a total pressure of 5 mbar at $220^{\circ} \mathrm{C} .112$ 25

Figure 15.Calculational characterization of fluxionality of free 13-atom gold clusters. Three-dimensional minimum energy structures of an Au13 cluster formed during the first 26 ps of a BornOppenheimer molecular dynamics simulation at $300 \mathrm{~K}$. Many structures were sampled numerous times within the first $80 \mathrm{ps}$ of the simulation at the sample temperature. A plot of the relative energy of these six structures demonstrates they are comparable to each other in energy, and the barriers associated with transformation between configurations are low-lying and less than $k T$ in magnitude $\left(2.48 \mathrm{~kJ} \mathrm{~mol}^{-1}\right)$. For larger particles (diameter $\sim 1 \mathrm{~nm}$ ), the core (bulk) atoms are static, whereas the surface atoms are fluxional. The calculations were performed for the clusters in vacuum. It is believed such structural fluctuations will be dampened in the presence of adsorbates and supports. ${ }^{113}$ 26

Figure 16.Schematic representation of spin-driven epoxidation reaction on Ag33. Calculations for propylene epoxidation on low-spin and high-spin $\mathrm{Ag} 33$ and of singlet and triplet $\mathrm{Ag}_{4} \mathrm{O}$ suggest that as the epoxidation reaction proceeds, a lower energy pathway is enabled via reaction on a high-spin magnetic structure that lowers the energy barrier as shown in the schematic (spin density shown in red on structures). 
Figure 17.(Top) Single nanoparticle trajectory for the reduction of resazurin to resorufin in the presence of hydroxylamine with single-turnover resolution catalyzed by a 6-nm gold nanoparticle electrostatically bound to a glass support. The individual waiting times, $\tau_{\text {off }}$ and $\tau_{\text {on }}$, represent the time averaged single-particle rate of particle formation and rate of product dissociation, respectively. It is apparent that the individual waiting times are not identical. (Bottom) The variances in $\tau_{\text {on }}$ and $\tau_{\text {off }}$ are believed to be associated with the fluxional behavior of the underlying surface atoms of the gold nanoparticle. For each nanoparticle, the temporal variation of its rate of turnover (catalysis) has contributions from the reaction rate changes in $\tau_{\text {off }}$ and $\tau_{\text {on. }}$. These two contributions can be evaluated for each nanoparticle by extracting the sequences of individual $\tau_{\text {off }}$ and $\tau_{\mathrm{on}}$ from its turnover trajectory and analyzing their autocorrelation functions $C_{\tau}(m)=$ $<\Delta \tau(0) \Delta \tau(m) / \Delta \tau^{2}>$, where $\tau$ is either $\tau_{\text {off }}$ or $\tau_{\text {on }}, m$ is the turnover index number in the sequences, and $\Delta \tau(m)=\tau(m)-<\tau>$. In the presence of activity fluctuations, $C_{\tau}(m) \geq 0$ and shows exponential decay behavior. The time constant associated with this decay is the activity fluctuation correlation time. The insets demonstrate that individual particles in a seemingly homogeneous population of 9$\mathrm{nm}$ gold nanoparticles have diverse activity fluctuation correlation times, indicating that individual nanoparticles can have very different surface restructuring time scales. The correlation between restructuring rates and activity fluctuations in catalysis is not direct but is inferred because the fluxional nature of the gold nanoparticles cannot be observed directly. Although this inference is consistent with our picture of the catalytic surface as a dynamic entity, UTEM observations during catalysis offer the prospect of enabling direct measurements of these fluctuation rates and correlations of these rates with fluctuations in either $\tau_{\mathrm{off}}$ or $\tau_{\mathrm{on}}{ }^{11}$ 28

Figure 18.In situ detection of redox reactivity of cerium by EELS: Ce $\mathrm{M}_{4,5}$ white line intensities in electron energy loss spectra are used to detect local changes in the cerium oxidation state in $\mathrm{CeO}_{2}$ nanoparticles during redox reactions. The intensity of the $\mathrm{Ce}_{5} \mathrm{M}_{5}$ increases relative to $\mathrm{M}_{4}$ during reduction and can be plotted against the oxidation state to obtain oxidation state directly from the white-line ratio. The technique can be used for doped ceria and can be extended to other transition metal oxides. ${ }^{115}$ 29

Figure 19.Grazing-incidence small-angle x-ray scattering (GISAXS) images of $\sim 9$-nm silver particles (a) at room temperature, from left to right: image recorded at the time of the inlet of the propyleneoxygen mixture, after $10 \mathrm{~min}$ of exposure and after $30 \mathrm{~min}$ of exposure. (b) Change of the scattering pattern during ramping of the temperature from 23 to $200^{\circ} \mathrm{C}$. The arrows indicate the angle of the most intense component of the directional scattering, which is indicative of changes in the faceting of the particles. The evolving asymmetry of the scattering pattern indicates the change of particle shape from oblate-like to spherical. ${ }^{22}$ 31

Figure 20.Fluxionality of a catalyst resting state observed in short-time AIMD simulation: (a) structure of $\mathrm{Rh}_{4}\left(\mathrm{BH}_{2} \mathrm{NMe}_{2}\right)_{8}{ }^{2+}\left(3^{\prime} \mathrm{H}_{0}{ }^{2+}\right)$ at $300 \mathrm{~K}$ shown at $25 \mathrm{ps}$ intervals. Methyl groups have been subtracted for clarity. The atom colors are as follows: N, blue; B, yellow; H, light blue; and Rh, gray. Key fluxional processes evident in the AIMD trajectory are illustrated schematically: (b) Rh4 cluster isomerization, (c) terminal-to-bridge ligand migration, and (d) terminal ligand binding isomerization and hydride transfer. The times indicated in parentheses in (b)-(d) are indicative of where this process may be seen in the configurations given in the molecular dynamics configurations presented in (a) ${ }^{118}$. 35

Figure 21 ............... Time-scale of dynamic processes in materials science (lower image ${ }^{120}$ ). 
Figure 22.Simulation results for CdTe solid-liquid phase equilibria where the melt interface is normal to the (111) CdTe interface. Shown in (a) are fine-scale density plots of atomic composition and order parameter. Observe the CdTe fractionation in (a) and the interface faceting in (b) in the atomic coordination model. ${ }^{122}$ 38

Figure 23. Atomistic models of liquid Te showing formation of solid nuclei in the liquid phase but not directly adjacent to the solid-liquid interface. Such clustering should be within the range of a welldesigned UTEM melting experiment and would provide valuable details for CZT solidification that now can only be obtained via modeling and simulation. ${ }^{125}$ 39

Figure 24......Schematic diagram of laser-pulse-induced interface mixing probed by UTEM. $\quad 40$

Figure 25.STEM image (left) and $\mathrm{Ti}_{2,3}$ EELS spectra obtained along the red line normal to the interface (right) for $\mathrm{LaAlO}_{3} / \mathrm{SrTiO}_{3}$ interface. The persistence of the Ti EELS peaks well into the $\mathrm{LaAlO}_{3}$ is due to Ti outdiffusion. No $\mathrm{Ti}^{3+}$ is seen along this line. ${ }^{131}$.......................... 41

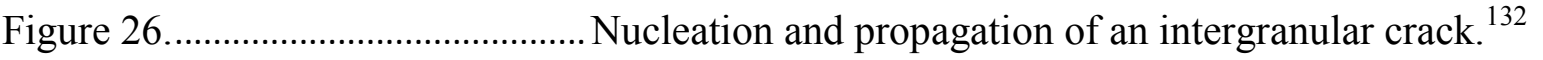
43

Figure 27 In situ testing of $100-\mathrm{nm}$-thick Al film using a MEMS device. ${ }^{135}$

Figure 28 Switching behavior of resistive memory based on $\mathrm{ZnO}$ doped with $\mathrm{Mn}^{137}$ 45

Figure 29.Cartoon of the electrochemically driven growth of a conducting silver filament within an insulating $\mathrm{ZnO}: \mathrm{Mn}$ layer of a resistive memory cell. Ag atoms from the top electrode are ionized and electromigrate to the bottom electrode, where they are reduced to neutral Ag and accumulate, forming a conducting filament spanning the layer. ${ }^{137}$ 46

Figure 30.Illustrations of nanoscale inhomogeneity arising in correlated electron systems. A) STM current image of antiferromagnetic $\mathrm{Ca}\left(\mathrm{Fe}_{1-\mathrm{x}} \mathrm{Co}_{\mathrm{x}}\right)_{2} \mathrm{As}_{2}$ acquired at $4.3 \mathrm{~K}^{169}$. The diagonal line is an orthorhombic twin boundary. The insets show fast Fourier transforms of the image contrast. The frame size is $96 \mathrm{~nm} \times 96 \mathrm{~nm}$. B) Distribution of superconducting gap values in as-grown BSCCO at $4.2 \mathrm{~K}^{170}$ The image size is $56 \mathrm{~nm} \times 56 \mathrm{~nm}$. 50

Figure 31.Fine grain microstructure of NFS with a high density of Ti-Y-O-rich nanoclusters. ${ }^{181} 52$

Figure 32.Molecular dynamics visualization of a $200 \mathrm{keV}$ collision cascade in pure Fe showing the peak of the damage at $0.67 \mathrm{ps}$ (a) after the initiation of the damage and near the end of the cascade at $14.5 \mathrm{ps}(\mathrm{b})$. The defects seen in (b) are representative of the freely migrating defects produced by the cascade that determine the extent of the radiation damage. For additional movies documenting radiation damage processes, refer to http://www-personal.umich.edu/ gsw/movies.html. ${ }^{182} 53$

Figure 33.High-energy heavy ion damage in $\mathrm{Gd}_{2} \mathrm{Ti}_{2} \mathrm{O}_{7}$ showing several nm-sized amorphous regions perpendicular to the ion track (into the photo). Amorphous regions are typical of damage in covalent or ionic materials. Note the lack of damage and disorder at the edge of the amorphous region. ${ }^{181}$ 54

Figure 34................... Cleared channels formed in oxygen-free high-conductivity copper. ${ }^{183}$ 55

Figure 35.Schematic of the LLNL DTEM I (left) and (right) image of the LLNL DTEM I as installed. ${ }^{29,30,193-196}$ 58

Figure 36. Single-shot, 15-nanosecond image of an $\mathrm{Au} / \mathrm{C}$ grating obtained from LLNL's DTEM I showing resolution less than $10-\mathrm{nm}^{193}$. 59 
Figure 37.This single-shot pulsed image of a stainless steel sample clearly shows that LLNL's DTEM I can image material defects such as dislocations and stacking faults with a single 15-nanosecond exposure. $^{196}$ 59

Figure 38. The evolution of single-shot DTEM columns from the earliest instrument to the proposed column for a UTEM. 64

Figure 39. Schematic of the (a) Plasmon assisted and (b) nano patterned approach to increasing gun brightness 65

Figure 40.Schematic of the proposed laser system for the single-shot UTEM: Dashed boxes indicate position of components for two upgrades ( $\mathrm{HG}=$ harmonic generation).

Figure 41.(a) Contrast transfer for an uncorrected TEM: green (standard Schottky FEG), red (ACTF), and black (PCTF): UTEM with $\alpha=30 \mathrm{mrad}$; blue: damping envelope of temporal coherence. (b) Contrast transfer for the spherically and chromatically corrected UTEM: red: PCTF for $\mathrm{C}_{5}=10 \mathrm{~mm}$; black: $\mathrm{ACTF}$ for $\mathrm{C}_{5}=10 \mathrm{~mm}$; blue: $\mathrm{ACTF}$ for $\mathrm{C}_{5}=1.0 \mathrm{~mm}$; green: $\mathrm{ACTF}$ for $\mathrm{C}_{5}=0.1 \mathrm{~mm}$ (note that these resolution limits assume that the space-charge effects caused by the lenses in the column do not degrade the resolution). .68 



\section{Appendix A}

\section{A.1 Microscopy Beyond the Femtosecond Time Scale: Exploration of Attosecond and Zeptosecond Phenomena in Matter}

The time scales for the motion of atoms in molecules and solids and for electronic excitation in nanostructures, molecules and atoms extends over six orders of magnitude, from picoseconds to attoseconds, as illustrated in Figure 42 and Figure 43. ${ }^{235}$ Although we know the order of magnitude of the time scales from simple arguments, actually probing the dynamics of atomic and electronic phenomena is a major challenge requiring innovative approaches to the generation of ultrafast pulses of photons and electrons. The last decade has seen significant progress in conceptual innovations for the generation and application of attosecond pulses to multi-electron dynamics in solids, molecules and atoms. ${ }^{235-244}$

Continuing advances in the science of attosecond photon pulses and femtosecond electron pulses will expose the full range of atomic and electronic dynamics in materials to quantitative study. The extension to subattosecond timescales (e.g., zeptosecond $\left(10^{-21} \mathrm{~s}\right)$ ) will enable a further journey beyond electrons into the nuclei of atoms. Mourou and Tajima ${ }^{245}$ highlight the inverse relationship between the laser pulse duration and coherent light intensity (Figure 44), suggesting that more powerful sources will lead to shorter pulses. Ipp ${ }^{246}$ discussed the possibility of creating double pulses of yoctosecond $\left(10^{-24} \mathrm{~s}\right)$ duration of $\mathrm{GeV}$ photon energy from the quark-gluon plasma (QGP) in non-central heavy-ion collisions (Figure 45). As the development of lasers, colliders, and experimental tools advances, the horizon is rich with possibilities

With the availability of controlled attosecond pulses, several microscopic techniques with attosecond resolution can be envisioned. According to Krausz and Ivanov, ${ }^{235}$ there are three emerging possibilities:

- Photoemission electron microscopy (PEEM): In this case, the continuous-wave UV or extreme UV (XUV) light source is simply replaced with pulses emitting this radiation in the form of attosecond bursts. $^{247}$

- Scanning tunneling microscopy (STM): Electron emission from the nanometer-sized tip may be confined to several hundred attoseconds by launching electrons with a cosine-shaped few-cycle light pulse via optical field ionization . ${ }^{248}$

- Ultrafast electron microscopy (UTEM): UTEM can potentially be advanced into the few-femtosecond and possibly sub-femtosecond regime ${ }^{249}$ by microwave ${ }^{227,250}$ and optical ${ }^{251}$ acceleration. 


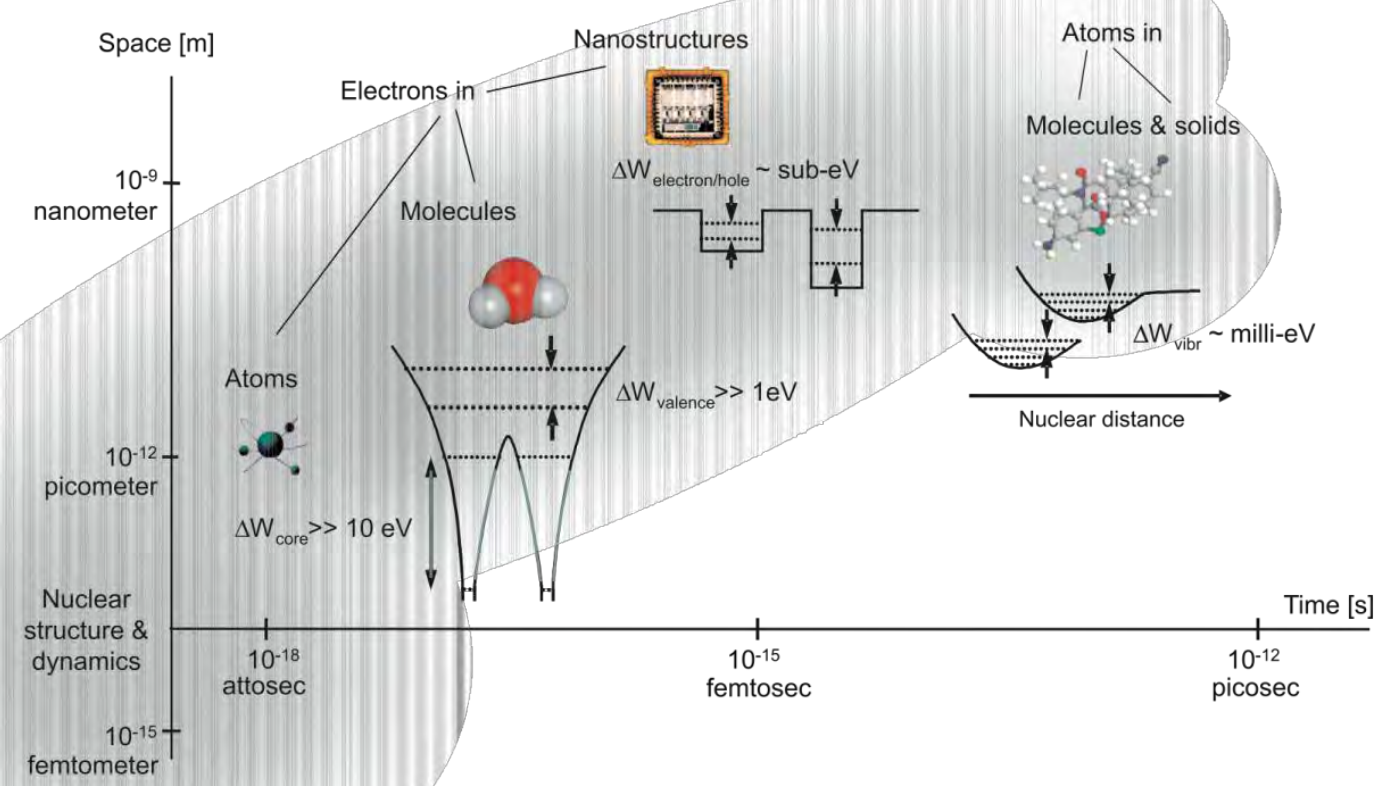

Figure 42: Characteristic length and time scales for structure and dynamics ${ }^{235}$

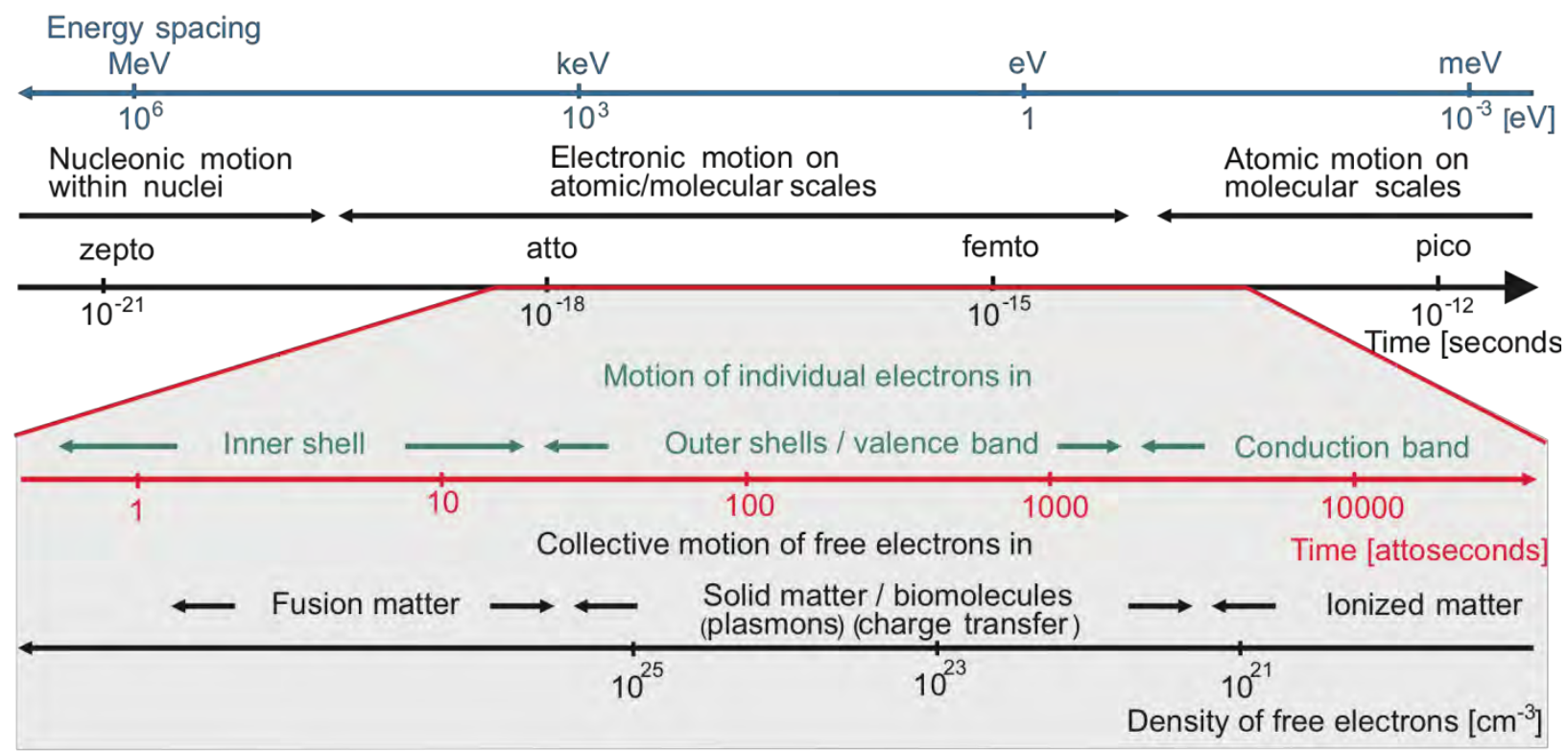

Figure 43: Top panel: Characteristic time scales for microscopic motion and its connection with energy spacing between relevant stationary states; Bottom panel: characteristic time scales for the motion of one or several electrons and for the collective motion of an electronic ensemble. ${ }^{235}$ 
The spatial resolution of these three approaches (nanometers of PEEM, angstroms of STM, and UTEM) can be combined with attosecond resolution in time, further opening up the imaging, diffraction and spectroscopy of electronic and nuclear motion with picometer resolution in space and attosecond resolution in time. Currently, the sub-femtosecond electron and photon pulses are limited to $100 \mathrm{eV}$ of energy. Advances in the attosecond sciences based on the relativistic light-electron interactions are expected to increase the energy dramatically, thus decreasing the duration of the probing particles in the future. With progress in these areas, movies of motions of the full range of atomic, electronic, and nuclear dynamics are within reach.

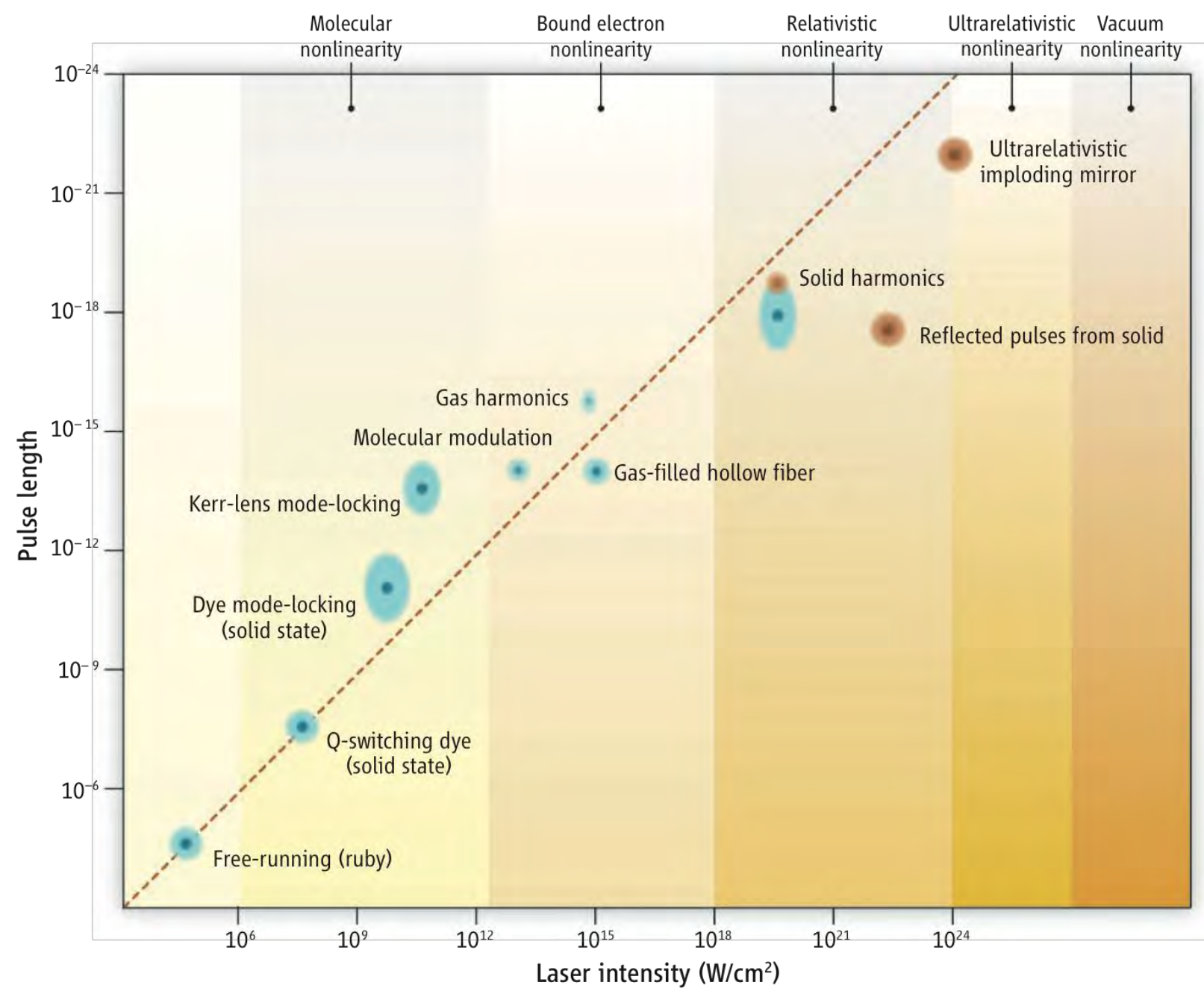

Figure 44: Pulse duration versus pulse intensity over 18 orders of magnitude (blue - experimental data; red simulation or theory). ${ }^{252}$ 


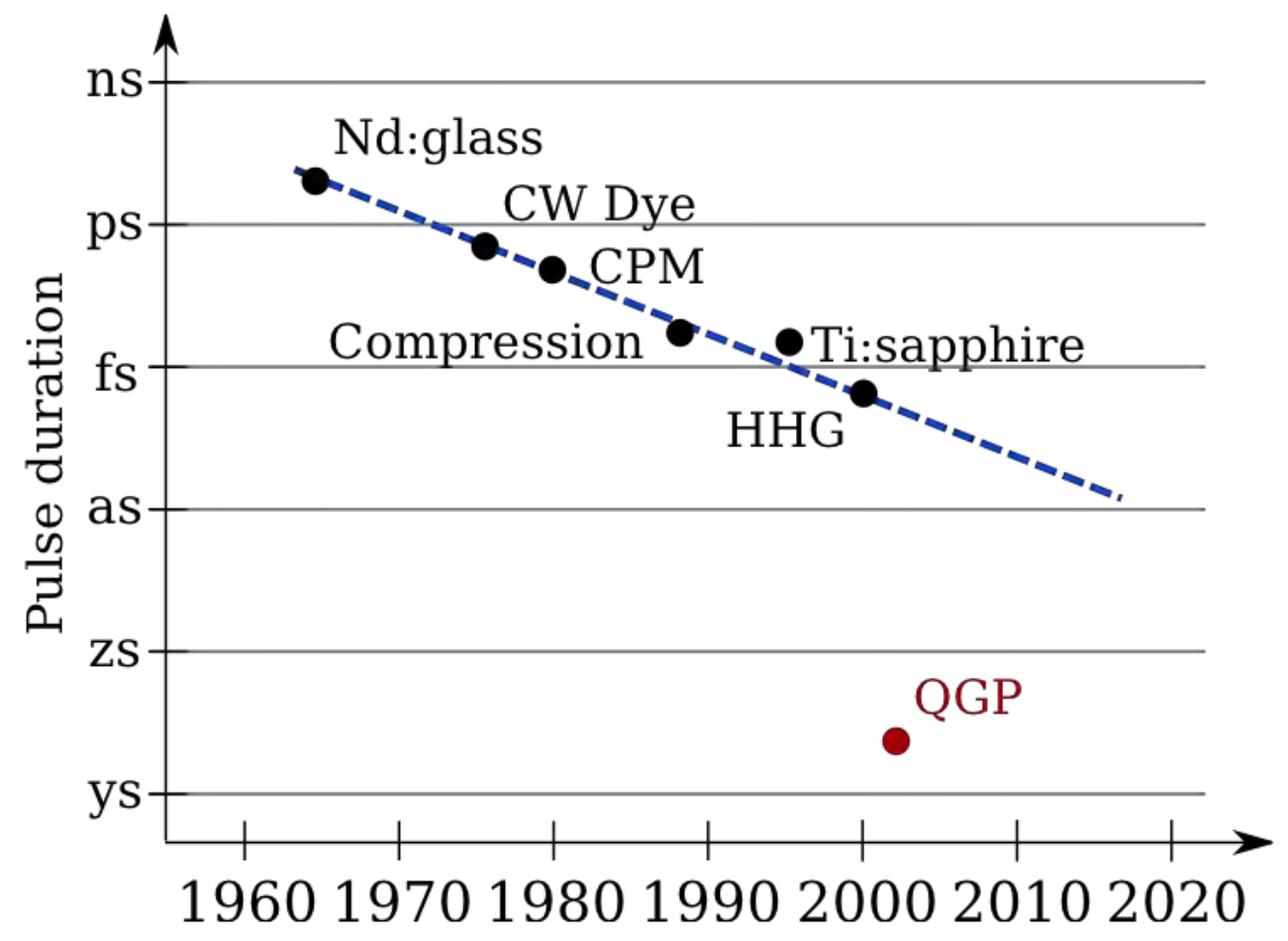

Figure 45: Historical perspective of laser pulse duration ${ }^{253}(\mathrm{CW}=$ continuous wave, $\mathrm{CPM}=$ colliding pulsemode, $\mathrm{HHG}=$ high-harmonic generation, and $\mathrm{QCP}=$ quark gluon plasma) ${ }^{246}$ 


\section{Appendix B}

\section{B.1 Program: Ultrafast TEM Workshop, June 14-15, 2011}

\begin{tabular}{|c|c|c|}
\hline TIME & TOPIC & LOCATION \\
\hline \multicolumn{3}{|c|}{ Tuesday, June 14, 2011} \\
\hline $8: 00-8: 45 \mathrm{am}$ & Welcome and Badging & EMSL Lobby \& ETB \\
\hline 8:45-9:00am & $\begin{array}{l}\text { Introduction - Allison Campbell, Associate Laboratory Director, } \\
\text { EMSL and Doug Ray, Associate Laboratory Director, FCSD }\end{array}$ & EMSL 1077 \\
\hline $9: 00-9: 15 \mathrm{am}$ & Overview - Bernd Kabius & EMSL 1077 \\
\hline $9: 45-10: 15$ am & Key note address - Material Science - George Crabtree & EMSL 1077 \\
\hline $10: 15-10: 45 \mathrm{am}$ & Key note address - Geochemistry - Mike Hochella & EMSL 1077 \\
\hline $10: 45-11: 15 \mathrm{am}$ & Key note address - Biology - Daniel Bond & EMSL 1077 \\
\hline $11: 15-11: 45 \mathrm{am}$ & Key note address - Theory \& Modeling - Warren Pickett & EMSL 1077 \\
\hline \multicolumn{3}{|c|}{ TEM Presentations: Session Chair - Christian Mailhiot } \\
\hline $1: 20-1: 40 \mathrm{pm}$ & $\begin{array}{l}\text { History and Status of In-Situ STEM/TEM: } \\
\text { Renu Sharma }\end{array}$ & EMSL 1077 \\
\hline $1: 40-2: 00 \mathrm{pm}$ & State of the Art of DTEM: Nigel Browning & EMSL 1077 \\
\hline
\end{tabular}




\begin{tabular}{|c|c|c|}
\hline TIME & TOPIC & LOCATION \\
\hline \multicolumn{3}{|c|}{ Breakout Session I: } \\
\hline \multirow[t]{4}{*}{$2: 20-6: 00 \mathrm{pm}$} & $\begin{array}{l}\text { Material Science } \\
\text { Session Lead: George Crabtree } \\
\text { Co-Chair: Libor Kovarik and SK Sundaram }\end{array}$ & EMSL 1077 \\
\hline & $\begin{array}{l}\text { Catalysis } \\
\text { Session Lead: Bruce Gates } \\
\text { Co-Chair: Chongmin Wang }\end{array}$ & EMSL 1044 \\
\hline & $\begin{array}{l}\text { UTEM - Technology - Components } \\
\text { Session Lead: Nigel Browning } \\
\text { Co-Chair: Andreas Schroeder }\end{array}$ & EMSL 2385 \\
\hline & $\begin{array}{l}\text { Biology/Geochemistry } \\
\text { Session Lead: John Zachara } \\
\text { Co-Chair: Alice Dohnalkova and James Evans }\end{array}$ & EMSL Board Room \\
\hline \multicolumn{3}{|c|}{ Wednesday, June 15, 2011} \\
\hline \multicolumn{3}{|c|}{ Presentations from each breakout session: Session chair - Bernd Kabius } \\
\hline $8: 00-8: 30 \mathrm{am}$ & Catalysis Session Lead: Bruce Gates & EMSL 1077 \\
\hline $8: 30-9: 00 \mathrm{am}$ & Biology/Geochemistry Session Lead: John Zachara & EMSL 1077 \\
\hline $9: 00-9: 30 \mathrm{am}$ & Material Science Session Lead: George Crabtree & EMSL 1077 \\
\hline $9: 30-9: 40 \mathrm{am}$ & $\begin{array}{l}\text { UTEM - Technology - Components } \\
\text { Session Lead: Nigel Browning }\end{array}$ & EMSL 1077 \\
\hline
\end{tabular}

\section{Breakout Session II:}




\begin{tabular}{|c|c|c|}
\hline TIME & TOPIC & LOCATION \\
\hline \multirow[t]{4}{*}{$9: 40-1: 30 p m$} & $\begin{array}{l}\text { Refine ideas - define topic areas-Discussion on the scientific } \\
\text { challenges presented. }\end{array}$ & \\
\hline & $\begin{array}{l}\text { Material Science } \\
\text { Session Lead: George Crabtree }\end{array}$ & EMSL 1077 \\
\hline & $\begin{array}{l}\text { Catalysis } \\
\text { Session Lead: Bruce Gates }\end{array}$ & EMSL 1044 \\
\hline & $\begin{array}{l}\text { Biology/Geochemistry } \\
\text { Session Lead: John Zachara }\end{array}$ & EMSL Board Room \\
\hline \multicolumn{3}{|c|}{ Breakout Session III: } \\
\hline $1: 30-5: 30 \mathrm{pm}$ & $\begin{array}{l}\text { Report writing: Small groups/individuals write up the results of } \\
\text { the previous sessions }\end{array}$ & EMSL 1077 \\
\hline
\end{tabular}




\section{Appendix C}

\section{C.1 Workshop participants}

Auciello, Orlando

Baer, Don

Banfield, Jill

Beyenal, Haluk

Bond, Daniel

Browning, Nigel

Bruemmer, Steve

Buck, Edgar

Chambers, Scott

Colby, Robert

Comolli, Luis

Crabtree, George

Dohnalkova, Alice

Edwards, Danny

Evans, James

Gates, Bruce

Goodwin, Shannon

Govind, Niri

Henager, Chuck

Hess, Nancy

Hess, Wayne

Hochella, Mike

Johnson, Brad

Kabius, Bernd

Karim, Ayman

King, David

Kovarik, Libor

Kurtz, Rick

Lea, Scott

Liu, Jingyue

Mailhiot, Christian

Marshall, Matt
Argonne National Laboratory

Pacific Northwest National Laboratory

University of California, Berkeley

Washington State University

University of Minnesota

University of California, Davis

Pacific Northwest National Laboratory

Pacific Northwest National Laboratory

Pacific Northwest National Laboratory

Pacific Northwest National Laboratory

Lawrence Berkeley National Laboratory

University of Illinois Chicago

Pacific Northwest National Laboratory

Pacific Northwest National Laboratory

University of California, Davis

University of California, Davis

Pacific Northwest National Laboratory

Pacific Northwest National Laboratory

Pacific Northwest National Laboratory

Pacific Northwest National Laboratory

Pacific Northwest National Laboratory

Virginia Tech

Pacific Northwest National Laboratory

Pacific Northwest National Laboratory

Pacific Northwest National Laboratory

Pacific Northwest National Laboratory

Pacific Northwest National Laboratory

Pacific Northwest National Laboratory

Pacific Northwest National Laboratory

University of Missouri St. Louis

Lawrence Livermore National Laboratory

Pacific Northwest National Laboratory 
McKinley, James

Meunier, Vincent

Miller, Toya

Mueller, Karl

Olszta, Matthew

Peden, Chuck

Phatak, Charudatta

Pickett, Warren

Rioux, Robert

Rodland, Karin

Saraf, Lax

Schreiber, Daniel

Schroeder, Andreas

Shaikh, Tanvir

Sharma, Renu

Shelton, Bill

Shuh, David

Squier, Tom

Sundaram, SK

Teller, Ray

Terminello, Lou

Thevuthasan, Theva

Thomas, Larry

Vajda, Stefan

Wang, Chongmin

Wang, Yong

Welp, Ulrich

Zachara, John
Pacific Northwest National Laboratory

Rensselaer Polytechnic Institute

Pacific Northwest National Laboratory

Pacific Northwest National Laboratory

Pacific Northwest National Laboratory

Pacific Northwest National Laboratory

Argonne National Laboratory

University of California, Davis

Penn State University

Pacific Northwest National Laboratory

Pacific Northwest National Laboratory

Pacific Northwest National Laboratory

University of Illinois at Chicago

Wadsworth Center

National Institute of Standards and Technology

Pacific Northwest National Laboratory

Lawrence Berkeley National Laboratory

Pacific Northwest National Laboratory

Alfred University

Pacific Northwest National Laboratory

Pacific Northwest National Laboratory

Pacific Northwest National Laboratory

Pacific Northwest National Laboratory

Argonne National Laboratory

Pacific Northwest National Laboratory

Pacific Northwest National Laboratory

Argonne National Laboratory

Pacific Northwest National Laboratory 